Review

\title{
Inorganic-Diverse Nanostructured Materials for Volatile Organic Compound Sensing
}

\author{
Muthaiah Shellaiah (D) and Kien Wen Sun * \\ Department of Applied Chemistry, National Chiao Tung University, Hsinchu 30010, Taiwan; \\ muthaiah1981@nctu.edu.tw \\ * Correspondence: kwsun@mail.nctu.edu.tw
}

check for

updates

Citation: Shellaiah, M.; Sun, K.W. Inorganic-Diverse Nanostructured Materials for Volatile Organic Compound Sensing. Sensors 2021, 21, 633. https://doi.org/10.3390/ s21020633

Received: 7 December 2020

Accepted: 14 January 2021

Published: 18 January 2021

Publisher's Note: MDPI stays neutral with regard to jurisdictional claims in published maps and institutional affiliations.

Copyright: (c) 2021 by the authors. Licensee MDPI, Basel, Switzerland. This article is an open access article distributed under the terms and conditions of the Creative Commons Attribution (CC BY) license (https:// creativecommons.org/licenses/by/ $4.0 /)$.

\begin{abstract}
Environmental pollution related to volatile organic compounds (VOCs) has become a global issue which attracts intensive work towards their controlling and monitoring. To this direction various regulations and research towards VOCs detection have been laid down and conducted by many countries. Distinct devices are proposed to monitor the VOCs pollution. Among them, chemiresistor devices comprised of inorganic-semiconducting materials with diverse nanostructures are most attractive because they are cost-effective and eco-friendly. These diverse nanostructured materials-based devices are usually made up of nanoparticles, nanowires/rods, nanocrystals, nanotubes, nanocages, nanocubes, nanocomposites, etc. They can be employed in monitoring the VOCs present in the reliable sources. This review outlines the device-based VOC detection using diverse semiconducting-nanostructured materials and covers more than 340 references that have been published since 2016.
\end{abstract}

Keywords: pollution; reliable nanosystems; VOC contamination; chemiresistor devices; device-based detection; sustainable application; nano-devices; diverse nanostructures

\section{Introduction}

Volatile organic compounds (VOCs) are the well-known cause of indoor pollution, which can harm the human health and leads to various disorders [1-3]. Long-time exposure to VOCs, such as phosgene, can lead to death and severe chronic diseases [4,5]. Another example is Benzene (a well-known carcinogen), which have the great potential to damage human tissues such as spleen, stomach, liver, kidneys, etc., and also affect the nervous, circulatory, immune, cardiovascular, and reproductive and respiratory systems [6,7]. Thus, to overcome such potential hazards of VOCs, numerous environmental safety agencies like Environmental Protection Agency (EPA), National Institute of Occupational Safety and Health (NIOSH), and European Agency for Safety and Health at Work (EU-OSHA), have fixated the accepted limit of certain VOCs down to sub-ppb concentrations [6]. To quantify such harmful VOCs, researchers focused to develop various sensory materials and to achieve the signals by means of fluorescent, electrochemical, or current-voltage (I-V) fluctuations, etc. [6-10]. Wherein, with the uniqueness and high sensitivity, chemiresistor device-based VOCs detection seems to be an interesting research topic [11,12]. In this track, nanomaterial-based semiconducting chemiresistor devices for VOCs monitoring are impressive in terms of sensitivity and selectivity $[13,14]$.

Materials with internal or external dimensions at nanoscale $(<100 \mathrm{~nm})$ are defined as nanomaterials $[15,16]$. They can be available in one to three dimensions (1D to 3D) with diverse structures like nanoparticles, nanocrystals, nanowires, nanocubes, nanosheets, nanotubes, nanosheets, nanocages, etc. [17]. Those nanostructured semiconducting materials play a vital role in sustainable applications such as conductivity studies, transistors, solar cells, healthcare diagnostics, sensors, and so forth [18-22]. Among them, utilization of semiconducting nanomaterials-based sensing of VOCs/gases seems to be 
more attractive $[23,24]$. Therefore, many reviews are available on $1 \mathrm{D}$ to $3 \mathrm{D}$ inorganicnanomaterials-based semiconducting gas/VOCs sensors [25-29]. Similarly, the utilization of semiconducting polymeric/hybrid/organic-based semiconducting nanomaterials were also proposed towards selective sensing of VOCs [30-37]. However, contrast to those polymeric/hybrid/organic nanostructures, inorganic semiconducting nanomaterials are highly admirable due to the low cost processing, durability, environment affordability, and high reproducibility, etc. [38-40]. Therefore, the effects of diverse nanostructured properties of inorganic semiconducting materials towards the determination of VOCs require more discussions. For example, Tin dioxide $\left(\mathrm{SnO}_{2}\right)$ seems to exist in diverse nanostructured forms, like nanoparticles, nanowires, hollow micro/nano-spheres, nanofibers, and cubahedra, with specific selectivity to different VOCs, such as acetone, ethanol, butanol, and toluene [38-43]. Thus, it is essential to gather information on consumption of diverse nanostructured materials in the device based detection of VOCs.

To this path, reviews on analytes quantification by means of electrochemical devices have already been reported by many researchers $[44,45]$. However, the information regarding the utilization of diverse inorganic-nanostructures in chemiresistor device-based VOCs determination, which is necessary for upcoming researchers, is currently deficient. Therefore, we deliver a comprehensive review on diverse nanostructured semiconducting nanomaterials that has been employed in device-based VOCs quantifications based on recent reports (more than 340 references published since 2016).

In this review, valuable information on diverse nanostructures employed in chemiresistor device-based VOCs detection and quantification (Figure 1) is revealed and discussed. Moreover, the mechanisms and effects of semiconducting nanostructures are clarified with charge/electron transport properties of those sensory nanomaterials. Lastly, synthesis of diverse nanostructured materials, advantages and disadvantages of those nanostructures, and their future scope towards VOCs quantification are summarized with justification.

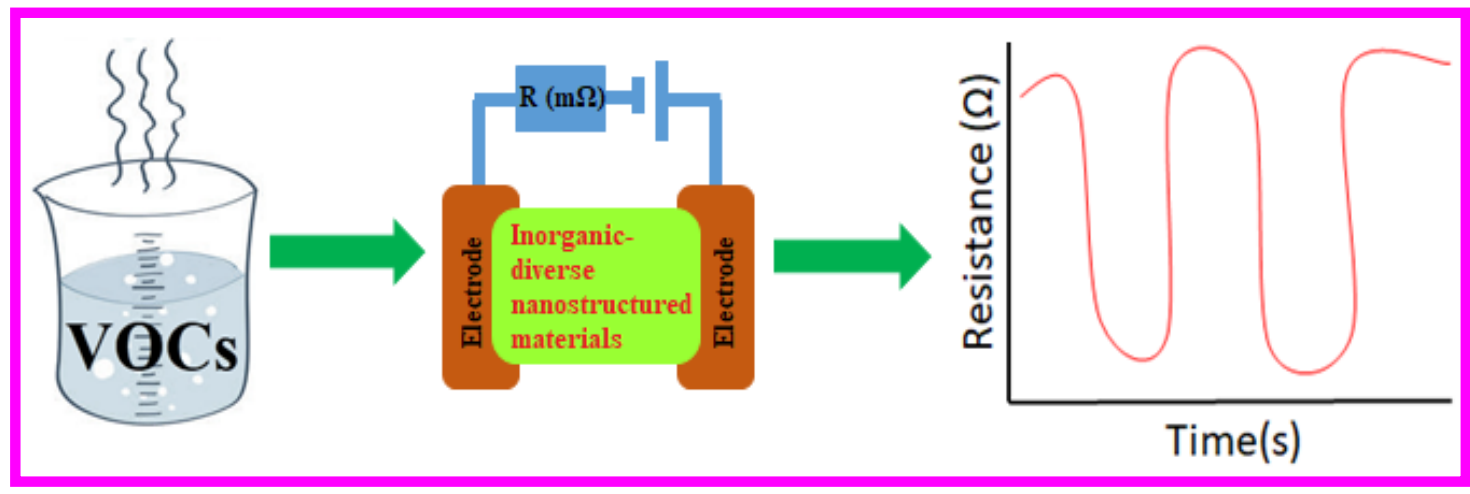

Figure 1. Schematic illustration of device based sensing of diverse nanostructures to volatile organic compounds (VOCs).

\section{Materials and Diverse Nanostructure Selections}

In general, design and development of semiconducting nanomaterial-based gas sensors need to follow some important rules as follows.

A. For device-based detection, the materials must have the unique p- or n-type semiconducting properties, which can be further boosted by combining with other materials to form a p-n or p-p or n-n heterojunction towards specific analyte quantification [46].

B. The selected nanomaterials must have large surface to enhance the adsorption of gaseous VOCs, which can induce signal responses, such as I-V fluctuations, electrochemical responses, fluorescent deviations, etc. However, for device-based sensing, signals are obtained as either I-V or electrochemical responses.

C. To attain the discrimination of diverse VOCs, different 1D to 3D nanostructures (nanoparticles/quantum dots, nanocrystals, nanorods/nanowires/nanoneedles, nanofibers/nanobelts, nanotubes, nanocubes, nanocages, nanowalls, nanosheets, 
nanoflakes/nanoplates, nanospheres, nanoflowers, porous-nanostructures, hierarchical nanostructures, and so on) can be adapted via optimizing their selectivity and sensitivity to specific analyte [47-58].

D. Diverse nanostructures can be developed by chemical synthesis, hydrothermal, chemical vapor deposition (CVD), combustion synthesis, sputtering, electrospinning, impregnation, sol-gel, solid-state reaction, hybrid composite synthesis, etc., to achieve specific sensitivity to the target VOC [47-58]. However, this should be done without affecting semiconducting properties of the proposed nanostructure, otherwise the sensitivity and reproducibility may be affected significantly.

E. Fabricated nanostructures on device must withstand different VOC exposures and at diverse humid/temperature conditions. Many devices may be affected by humidity of the environment and lead to malfunction, thereby special attention should be paid to the effect of humidity on the sensory devices. Similarly, certain semiconducting/hybrid materials can function at either higher or lower temperatures. Therefore, justification of operating temperature is a must for the sensory devices.

\section{Diverse Nanostructures in Acetone Detection}

Detection of acetone vapor by diverse nanostructures and materials follows the general mechanism of adsorption of volatile vapor over the surface of the substrate, resulting to the release of electrons and induce resistance changes $\left(R_{a} / R_{g} ; R_{a}\right.$ and $R_{g}$ are resistance without and with the target gas). During the injection of acetone vapor in the gaseous chamber consist of diverse nanostructured materials, the vapor reacts with the oxygen species that is already adsorbed, as descripted in the Equations (1) and (2), which releases electrons to be detected as sensor signal $\left(R_{a} / R_{g}\right)$.

$$
\begin{gathered}
\left(\mathrm{CH}_{3} \mathrm{COCH}_{3}\right)_{\mathrm{g}} \rightarrow\left(\mathrm{CH}_{3} \mathrm{COCH}_{3}\right)_{\mathrm{ads}} \\
\left(\mathrm{CH}_{3} \mathrm{COCH}_{3}\right)_{\mathrm{ads}}+8 \mathrm{O}^{-} \rightarrow 3 \mathrm{CO}_{2}+3 \mathrm{H}_{2} \mathrm{O}+8 \mathrm{e}^{-}
\end{gathered}
$$

The sensitivity can be varied depending on the adsorption surface area, which might be the concern with diverse nanostructures towards VOCs detection. Such quantifications can also be significantly affected by the semiconducting properties, such as p-type, n-type, $\mathrm{p}-\mathrm{n}, \mathrm{p}-\mathrm{p}$, and $\mathrm{n}-\mathrm{n}$ heterojunctions. Thus, information on miscellaneous nanostructured materials that has been reported in acetone detection and sensitivities are outlined below.

Among various nanostructures, the semiconducting nanoparticles (NPs) are capable of providing uniform surface for acetone adsorption to deliver unique resistance changes at certain temperatures. To this path, $\mathrm{TiO}_{2} \mathrm{NPs}, \alpha-\mathrm{Fe}_{2} \mathrm{O}_{3} \mathrm{NPs}, \mathrm{Mn} @ Z n O$ NPs (MZO NPs; $\mathrm{p}+\mathrm{n}$ interlock, field effect transistor), Pt-decorated Al-doped $\mathrm{ZnO}$ (Pt-AZO NPs), AZO NPs, B-TiO ${ }_{2} @ \mathrm{Ag}$ NPs, $\mathrm{La}_{1-\mathrm{x}} \mathrm{Y}_{\mathrm{x}} \mathrm{MnO}_{3-\delta} \mathrm{NPs}$, and $\mathrm{Bi}_{1-\mathrm{x}} \mathrm{La}_{\mathrm{x}} \mathrm{FeO}_{3}$ NPs were consumed in selective device-based quantification of acetone with part per billion/parts per million (ppb/ppm) detection limits (LODs) [59-66]. These nanoparticles are generally synthesized via hydrothermal ( $\mathrm{TiO}_{2}$ NPs, MZO NPs, Pt-AZO NPs, AZO NPs, and B-TiO $@$ @Ag NPs), reverse micro-emulsion ( $\left.\alpha-\mathrm{Fe}_{2} \mathrm{O}_{3} \mathrm{NPs}\right)$, and sol-gel $\left(\mathrm{La}_{1-\mathrm{x}} \mathrm{Y}_{\mathrm{x}} \mathrm{MnO}_{3-\delta} \mathrm{NPs}\right.$ and $\mathrm{Bi}_{1-\mathrm{x}} \mathrm{La}_{\mathrm{x}} \mathrm{FeO}_{3}$ NPs) methods that operate between 250 and $500{ }^{\circ} \mathrm{C}$ (as summarized in Table 1 ). Among these nanoparticles, Pt-AZO NPs are quite unique with a $R_{a} / R_{g}$ response of 421 , due to the doping and decoration of $\mathrm{Al}$ and $\mathrm{Pt}$, correspondingly. Wherein, decoration of $\mathrm{Pt}$ enhances the sensor responses via increased $\mathrm{O}_{2}$ adsorption (which reacts with acetone to release more electrons) on the surface, as shown in Figure 2. However, the main drawback of this work is its operating temperature $\left(450{ }^{\circ} \mathrm{C}\right)$.

Similar to NPs, nanocrystalline materials were also involved in volatile acetone discrimination. For example, Zhang et al. developed the Pd-doped $\mathrm{SmFe}_{0.9} \mathrm{Mg}_{0.1} \mathrm{O}_{3}$ nanocrystals by a sol-gel tactic and utilized in acetone detection under light illumination at $220{ }^{\circ} \mathrm{C}$ [67]. Wherein, the $\mathrm{Pd}: \mathrm{SmFe}_{0.9} \mathrm{Mg}_{0.1} \mathrm{O}_{3}$ displayed an impressive response of 7.16 (for $0.5 \mathrm{ppm}$ ) with a LOD of $0.01 \mathrm{ppm}$. To this track, hydrothermally synthesized $\mathrm{WO}_{3}$ nanocrystals were also displayed a good response $\left(\mathrm{R}_{\mathrm{a}} / \mathrm{R}_{\mathrm{g}}=3.8 ; 0.25 \mathrm{ppm}\right)$ at $320{ }^{\circ} \mathrm{C}$ 
with a satisfactory LOD of $0.075 \mathrm{ppm}$ [68]. Moreover, Rhodium (Rh) additive in the solvothermally synthesized $\mathrm{TiO}_{2}$ nanocrystals $\left(\mathrm{TiO}_{2}-5 \mathrm{Rh}\right)$ slightly improved the sensor response $\left(\mathrm{R}_{\mathrm{a}} / \mathrm{R}_{\mathrm{g}}=9.6 ; 50 \mathrm{ppm}\right)$ at $300{ }^{\circ} \mathrm{C}$, as shown in Figure 3 [69]. However, this work still needs further optimization on the operating temperature and LOD. Considering the acetone quantitation, nanowires (NWs) were fabricated to afford device-based sensors. Functionalization or attachment of certain materials on the surface of NWs may enhance the sensitivity.

(a)

Air

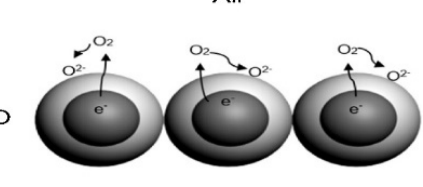

Spillover

(b)

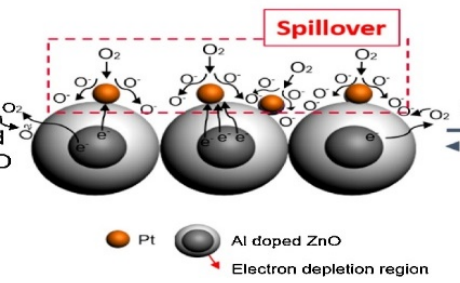

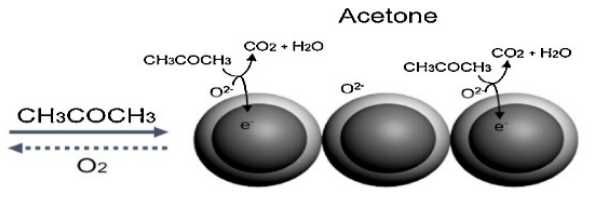

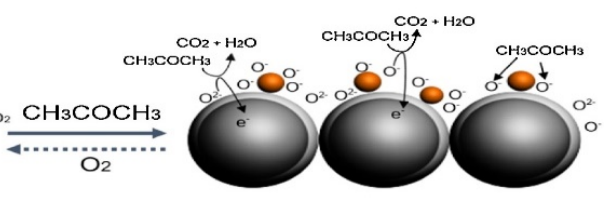

Figure 2. Schematic illustrating the sensing reaction mechanism of (a) Al-doped ZnO (AZO) and (b) Pt-decorated AZO (Pt-AZO) sensors in air and acetone (Reproduced with the permission from Ref. [62]).
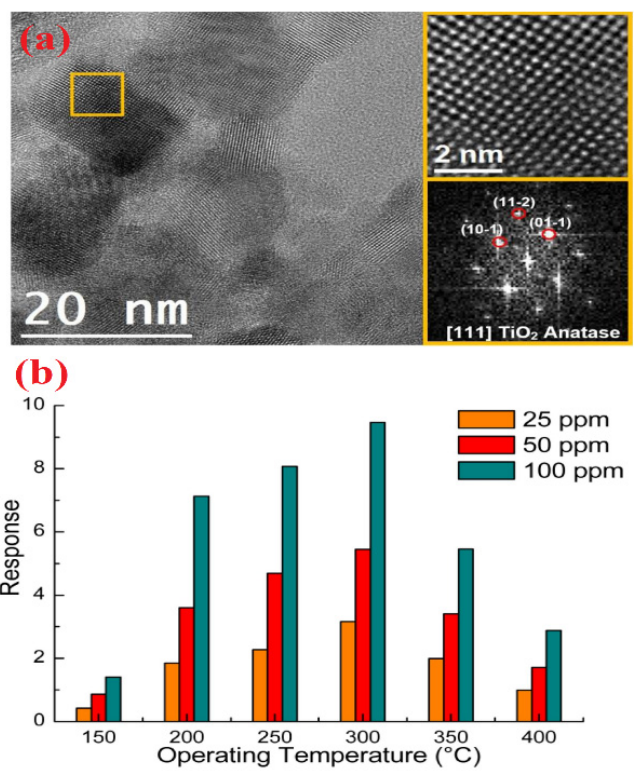

Figure 3. (a) HRTEM micrograph of the $400{ }^{\circ} \mathrm{C} \mathrm{TiO}_{2}-5 \mathrm{Rh}$ sample, detail of the orange squared region and its corresponding power spectrum; (b) Comparison between the $\mathrm{TiO}_{2}-5 \mathrm{Rh}$ acetone responses at various operating temperatures. The typical volcano behavior can be observed (Reproduced with the permission from Ref. [69]).

To this light, Kim et al. and Singh et al. demonstrated an exceptional acetone sensitivity (refer to Table 1) of $\mathrm{Co}_{3} \mathrm{O}_{4}$ NPs modified $\mathrm{SnO}_{2} \mathrm{NWs}$ and self-assembled monolayer (SAM) functionalized $\mathrm{ZnO}$ NWs $[70,71]$. These sensory materials can be synthesized via vaporliquid-solid (VLS), sol-gel, and thermal annealing processes to detect acetone with an LOD of $0.5 \mathrm{ppm}$. Additionally, p-n heterojunction NWs were proposed with $\mathrm{ZnO}$ branched p-CuxO@n-ZnO NWs (fabricated by hydrothermal method and atomic layer deposition) for acetone sensing [72]. Wherein, the sensor operated at $250^{\circ} \mathrm{C}$ and displayed low sensor 
responses of 3.39-6.38 (5-50 ppm), as depicted in Figure 4. Therefore, the device requires further optimization to attain a higher response to achieve excellent performance.

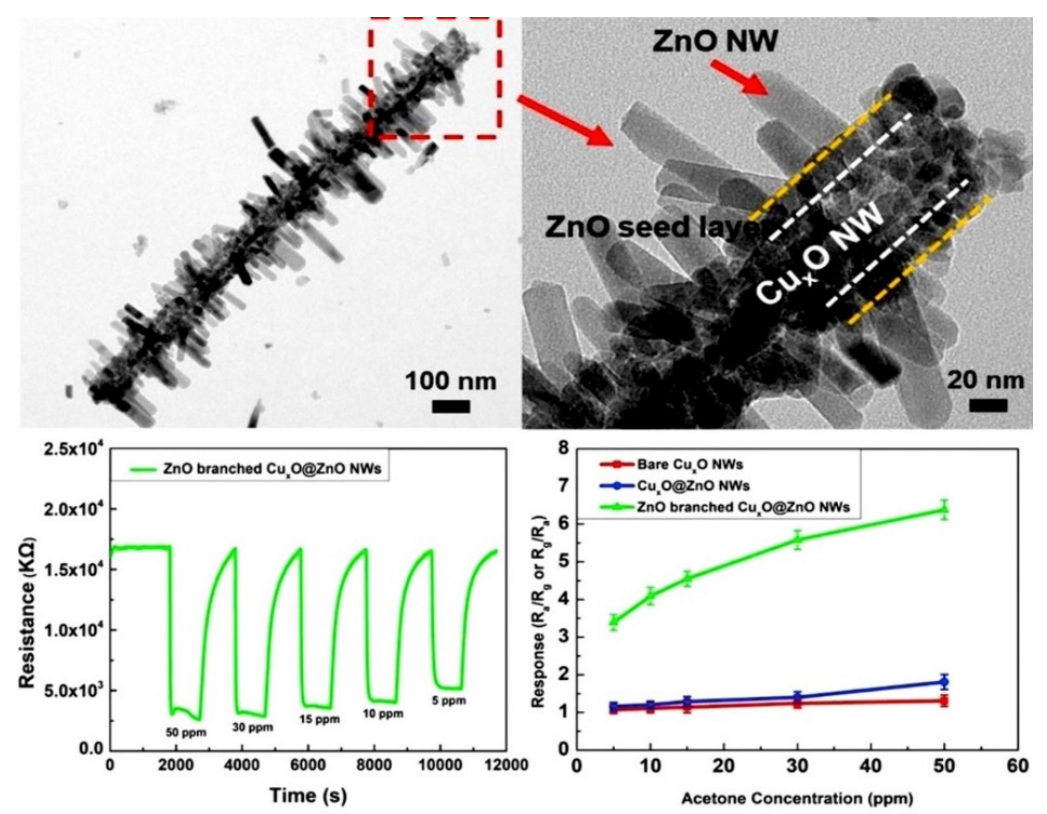

Figure 4. TEM image of $\mathrm{ZnO}$ branched $\mathrm{p}-\mathrm{CuxO} @ \mathrm{n}-\mathrm{ZnO}$ heterojunction nanowires and its sensor responses to acetone (Reproduced with the permission from Ref. [72]).

Discrimination of acetone was also demonstrated by hydrothermally/chemically synthesized nanorods (NRds) and nanoneedles at certain operating temperatures with good response/recovery time, as seen in Table 1 [73-77]. Among these nanorods $(\mathrm{Cr}$ doped $\mathrm{ZnO} N R d s, \mathrm{SnS}_{2} \mathrm{NRds}$, $\mathrm{Au} / \mathrm{Pd}$-doped $\mathrm{ZnO} \mathrm{NRds}$, and $\alpha-\mathrm{Fe}_{2} \mathrm{O}_{3} / \mathrm{NiO} \mathrm{NRds}$ ), the $\alpha-\mathrm{Fe}_{2} \mathrm{O}_{3}$ NPs-doped NiO NRds [76] displayed a high response of 290 for $100 \mathrm{ppm}$ acetone at $280^{\circ} \mathrm{C}$ with a LOD of approximately $5 \mathrm{ppm}$. This might be due to its p-n heterojunction property, but the operation temperature of the sensor operation requires further reduction. Because the Ag-doped $\mathrm{ZnO}$ nanoneedles [77] did not display any exceptional response $\left(\mathrm{R}_{\mathrm{a}} / \mathrm{R}_{\mathrm{g}}=30.233\right.$; for $200 \mathrm{ppm}$ at $\left.370{ }^{\circ} \mathrm{C}\right)$; therefore, they did not play an important role in volatile acetone detection. Devices with hydrothermally synthesized nanoarrays, such as La-doped $\mathrm{SnO}_{2}$, hybrid $1 \mathrm{D} / 2 \mathrm{D} \alpha-\mathrm{Fe}_{2} \mathrm{O}_{3}-\mathrm{SnO}_{2}$, and $\mathrm{ZnTiO}_{3}$ were engaged in acetone gas estimation [78-80]. Wherein, in contrast to other nanoarrays, $\mathrm{ZnTiO}_{3}$ nanoarrays [80] showed exceptional sensor responses of $78 / 94$ for $12.5 \mathrm{ppm}$ at $45^{\circ} \mathrm{C} / 350^{\circ} \mathrm{C}$ with the LODs of 0.09 and $0.01 \mathrm{ppm}$, correspondingly. This work is impressive because its operation at dark and light conditions can be completed with less than $3 \mathrm{~min}$ response/recovery time.

Volatile acetone quantitation was also authenticated by many semiconducting nanofibers fabricated by hydrothermal, electrospinning, and calcination tactics. Nanofibers like Ag-decorated $\mathrm{SnO}_{2}, \mathrm{PrFeO}_{3}, \mathrm{Pt}-\mathrm{ZnO}-\mathrm{In}_{2} \mathrm{O}_{3}, \mathrm{Au} @ \mathrm{WO}_{3}-\mathrm{SnO}_{2}$, In-doped $\mathrm{ZnSnO}_{3}, \mathrm{ZnO}$, and $\mathrm{Ru}$-doped $\mathrm{SnO}_{2}$ showed selective sensitivity to acetone at various operating temperatures $\left(150-300{ }^{\circ} \mathrm{C}\right)$ [81-87]. Among them, $\mathrm{Au} @ \mathrm{WO}_{3}-\mathrm{SnO}_{2}$ [84] developed by Shao et al. displayed a high response of 196.1 for $10 \mathrm{ppm}$ at $150^{\circ} \mathrm{C}$, with an estimated LOD of $<0.5 \mathrm{ppm}$ and response/recovery time of $<2 \mathrm{~min}$. In this track, functionalized/sensitized/assembled/ decorated nanotubes (NTs) and multi-walled carbon nanotubes (MWCNTs) were reported by many research groups towards acetone sensing. $\mathrm{Pd} / \mathrm{Pt}$ functionalized $\mathrm{SnO}_{2} \mathrm{NTs}$, PdO@ZnO- $\mathrm{SnO}_{2}$ NTs, $\alpha-\mathrm{Fe}_{2} \mathrm{O}_{3}$ NRds-MWCNTs, $\mathrm{Co}_{3} \mathrm{O}_{4}-\mathrm{MWCNTs}, \mathrm{Pt}-\mathrm{CuFe}{ }_{2} \mathrm{O}_{4}$ NTs, $\mathrm{Pd} @ \mathrm{WO}_{3}-\mathrm{SnO}_{2} \mathrm{NTs}$, and ZnO-Decorated In/Ga Oxide NTs (synthesized by hydrothermal, encapsulation, electrospinning, and soaking) were reported for exceptional acetone detection at various operating temperatures $\left(225-400{ }^{\circ} \mathrm{C}\right)$ [88-94]. These decoration of certain catalytic substrates may enhance acetone selectivity by varying the operating temperature. For example, Pt or Pd loaded multidimensional $\mathrm{SnO}_{2}$ NTs [88] displayed an exceptional 
sensitivity to acetone $\left(\mathrm{R}_{\mathrm{a}} / \mathrm{R}_{\mathrm{g}}=93.55\right.$ for $5 \mathrm{ppm}$ at $\left.350^{\circ} \mathrm{C}\right)$ with a LOD of $<1 \mathrm{ppm}$. Similarly, $\mathrm{PdO}-\mathrm{ZnO}$ composite on hollow $\mathrm{SnO}_{2}$ NTs [89] showed a good sensor response to acetone even at low concentration (Rair $/ \operatorname{Rgas}=5.06$ for $1 \mathrm{ppm}$ at $400{ }^{\circ} \mathrm{C}$ ) with a LOD of $0.01 \mathrm{ppm}$. This sensor operates under higher humidity $(95 \% \mathrm{RH})$ than that of others, thereby is quite noticeable. The sensitivity can be enhanced by forming multiple heterojunctions and by chemical sensitization effect of nanocatalyst.

For instance, the sensitivity of $\mathrm{Pd} @ \mathrm{WO}_{3}-\mathrm{SnO}_{2} \mathrm{NTs}$ [93] to acetone was enhanced by multiple heterojunctions formation $\left(\mathrm{W}_{\mathrm{O}}-\mathrm{SnO}_{2} \mathrm{n}-\mathrm{n}\right.$ junctions, $\mathrm{PdO}-\mathrm{SnO}_{2}$, and $\mathrm{PdO}-\mathrm{WO}_{3}$ $\mathrm{p}-\mathrm{n}$ junctions) and by the sensitization effect of $\mathrm{Pd}$ nanocatalyst. Doped $\mathrm{SnO}_{2}$ nanobelts were employed as sensor materials for the discrimination of acetone with LODs down to sub-ppm level [95,96]. Li et al., and Chen et al. reported the development of Y-or Eu-doped $\mathrm{SnO}_{2}$ nanobelts by thermal evaporation tactic above $1350{ }^{\circ} \mathrm{C}$ and utilized them in acetone sensing with verified enhanced performance (refer to Table 1) than those of pure $\mathrm{SnO}_{2}$ nanobelts. Volatile acetone gas detection has been well demonstrated by nanocubes derived from p-type $\mathrm{Co}_{3} \mathrm{O}_{4}$, hybrid $\mathrm{In}_{2} \mathrm{O}_{3} @ \mathrm{RGO}, \mathrm{Ag}$ functionalized $\mathrm{ZnSnO}_{3}, \mathrm{ZnO}-\mathrm{CuO}$ p-n heterojunction, p-type $\mathrm{NiFe}_{2} \mathrm{O}_{4}, \mathrm{NiO} / \mathrm{ZnO}$ composite, and $\mathrm{MOF}$ derived- $\mathrm{ZnO} / \mathrm{ZnFe}_{2} \mathrm{O}_{4}$ [97-103]. Among them, $\mathrm{ZnO}-\mathrm{CuO}$ p-n heterojunction displayed a good sensor response of 11 to 1 ppm acetone at $200{ }^{\circ} \mathrm{C}$ with an impressive LOD of $0.009 \mathrm{ppm}$. Moreover, the material has a stable response up to 40 days, thereby becomes a notable candidate. Similarly, hybrid $\mathrm{In}_{2} \mathrm{O}_{3} @ \mathrm{RGO}$ nanocubes [98] showed discriminative sensing to acetone and formaldehyde at $175^{\circ} \mathrm{C}$ and $225^{\circ} \mathrm{C}$, respectively, but the interference studies were still lacking in this report.

The acetone sensing capability of nanocages has been established by PdO functionalized $\mathrm{Co}_{3} \mathrm{O}_{4}$ hollow nanocages, $\mathrm{ZnO} / \mathrm{ZnFe}_{2} \mathrm{O}_{4}$ hollow nanocages, $\mathrm{PdO}$ functionalized $\mathrm{NiO} / \mathrm{NiCo}_{2} \mathrm{O}_{4}$ truncated nanocages, and $\mathrm{Ag} @ \mathrm{CuO}-\mathrm{TiO}_{2}$ hollow nanocages [104-107]. Wherein, $\mathrm{PdO}$ acted as a catalyst (in $\mathrm{PdO}$ functionalized $\mathrm{Co}_{3} \mathrm{O}_{4}$ hollow nanocages and $\mathrm{PdO}$ functionalized $\mathrm{NiO} / \mathrm{NiCO}_{2} \mathrm{O}_{4}$ truncated nanocages) to enhance the sensitivity and $\mathrm{PdO} @ \mathrm{Co}_{3} \mathrm{O}_{4}$ hollow nanocages [104] displayed a response of 2.51 to 5 ppm acetone at $350{ }^{\circ} \mathrm{C}$ with a LOD of 0.1 ppm. Moreover, $\mathrm{PdO} @ \mathrm{Co}_{3} \mathrm{O}_{4}$ hollow nanocages can be operable at $90 \%$ humid condition. Next, $\mathrm{ZnO} / \mathrm{ZnFe}_{2} \mathrm{O}_{4}$ hollow nanocages [105] also detect the acetone gas to certain extend $\left(\mathrm{R}_{\mathrm{a}} / \mathrm{R}_{\mathrm{g}}=25.8\right.$ for $100 \mathrm{ppm}$ at $\left.290{ }^{\circ} \mathrm{C}\right)$, but require optimization on operating temperature. On the contrary, multicomponent $\mathrm{Ag} @ \mathrm{CuO}-\mathrm{TiO}_{2}$ hollow nanocages [107] enhance the acetone sensing at low operating temperature $\left(R_{a} / R_{g}=6.2\right.$ for $100 \mathrm{ppm}$ at $200^{\circ} \mathrm{C}$ ) with a calculated LOD of $\sim 1 \mathrm{ppm}$, as illustrated in Figure 5. However, more focus is required to improve the response.

Researchers synthesized various nanosheets (NShs) by means of post-thermal, hydrothermal, impregnation, liquid exfoliation, precipitation, and multistep approaches and applied them in gaseous acetone quantification. $\mathrm{Co}_{3} \mathrm{O}_{4} \mathrm{NSh}, \mathrm{ZnO} \mathrm{NSh}, \mathrm{SnO}_{2} / \mathrm{Fe}_{2} \mathrm{O}_{3}$ multilayer NShs, NiO NShs, and F-doped $\mathrm{TiO}_{2}$ NShs were engaged in acetone sensing at different operating temperatures as summarized in Table 1 [108-112]. In particular, F-doped $\mathrm{TiO}_{2}$ NShs grown on Ti foam [112] function linearly in the detection range of 25-800 ppm at $25{ }^{\circ} \mathrm{C}$ and are stable at divers humid conditions (20-90\% RH), thereby become exceptional material for acetone detection. Subsequently, materials with nanowalls were utilized in acetone sensory, which showed the unique advantages of wide surface area for volatile gas adsorption. $\mathrm{Nb}$-doped $\mathrm{ZnO}$ nanowalls (synthesized by radio-frequency (RF) magnetron sputtering), $\mathrm{CuO}$ nanowalls (from Oxidation of $\mathrm{Cu}$ foil in aqueous $\mathrm{NH}_{4} \mathrm{OH}$ ), $\mathrm{NiO}$ nanowalls (from chemical bath deposition (CBD), and $\mathrm{ZnO}$ deposited carbon nanowalls (from microwave plasma-enhanced chemical vapor deposition (MPECVD) were reported as acetone sensors with extensive selectivity [113-116]. Herein, Nb-doped ZnO nanowalls [113] operate at $200{ }^{\circ} \mathrm{C}$ and deliver a high response of 89.13 (for $100 \mathrm{ppm}$ ) with good linear response between 20 and 100 ppm. Moreover, $\mathrm{NiO}$ nanowalls [115] exhibit exceptional response to acetone $\left(R_{\mathrm{a}} / \mathrm{R}_{\mathrm{g}} \geq 30\right.$; for $10 \mathrm{ppm}$ at $\left.250{ }^{\circ} \mathrm{C}\right)$ with a LOD of $0.2 \mathrm{ppm}$ and exhibit the dynamic response as shown in Figure 6. Similarly, Choi et al. established wide surface area interaction of acetone to $\mathrm{ZnO}$ deposited carbon nanowalls [116]; therefore, development of such nanowalls-based VOCs sensors are highly anticipated. 

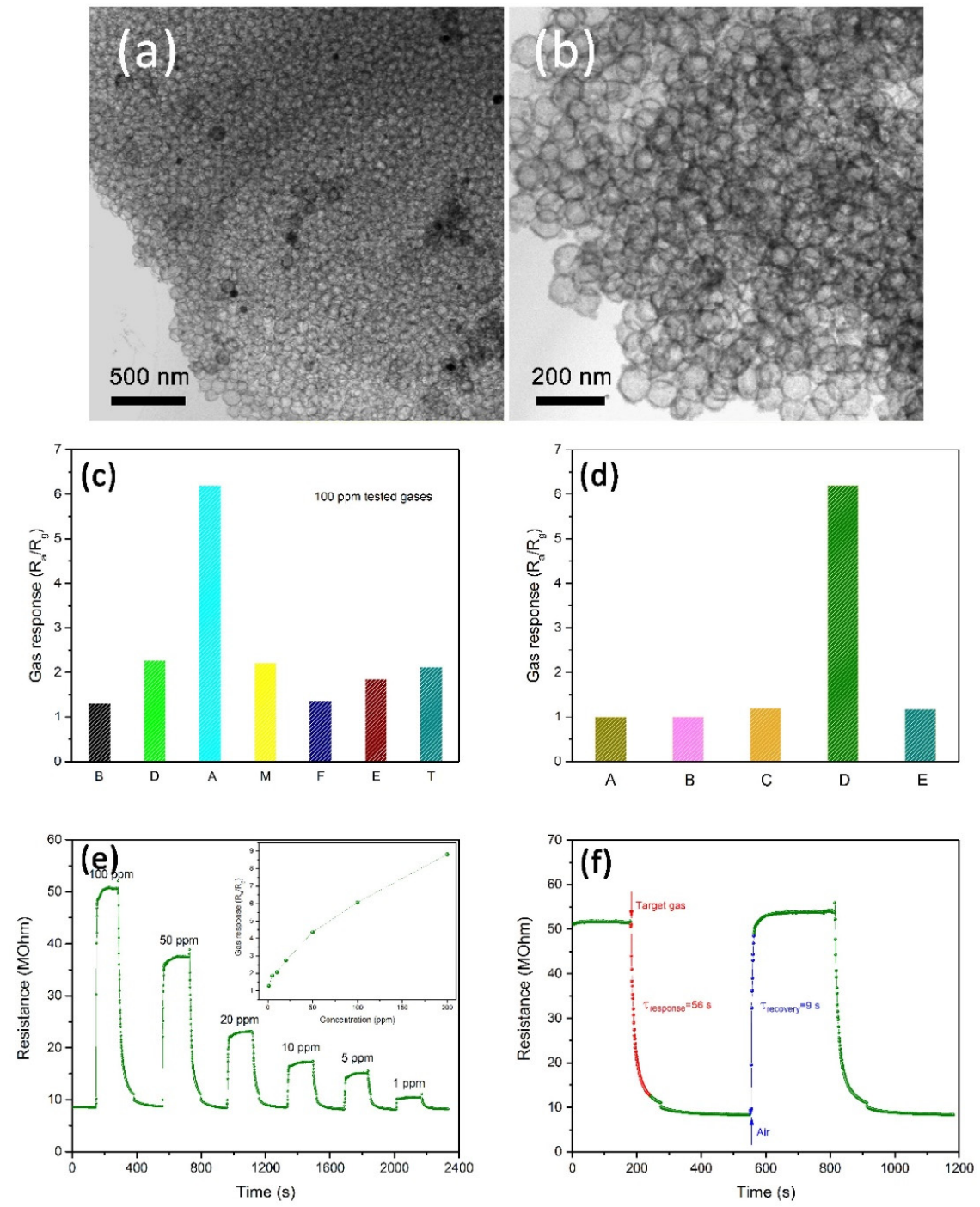

Figure 5. (a,b) The TEM images of $\mathrm{Ag} @ \mathrm{CuO}-\mathrm{TiO}_{2}$ hollow nanocages; (c) Gas responses to 100 ppm various target gases at $200{ }^{\circ} \mathrm{C}(\mathrm{B}$, benzene; $\mathrm{D}$, dimethylbenzene; $\mathrm{A}$, acetone; $\mathrm{M}$, methanol; $\mathrm{F}$, formaldehyde; E, ethanol; T, toluene); (d) Gas responses of different compositions to $100 \mathrm{ppm}$ acetone at $200{ }^{\circ} \mathrm{C}$ (A-D, A: Ag@CuO; B: $\left.\mathrm{TiO}_{2} ; \mathrm{C}: \mathrm{CuO}-\mathrm{TiO}_{2} ; \mathrm{D}: \mathrm{Ag} @ \mathrm{CuO}-\mathrm{TiO}_{2}\right)$ and $375^{\circ} \mathrm{C}\left(\mathrm{E}: \mathrm{TiO}_{2}\right)($ e) Responses vs. acetone concentrations at $200{ }^{\circ} \mathrm{C}$; (The inset figure is the linear relationship between response and concentration) (f) Response and recovery curves to $100 \mathrm{ppm}$ acetone at $200{ }^{\circ} \mathrm{C}$ (Reproduced with the permission from Ref. [107]).

Towards acetone selective sensing, nanoflakes were incorporated in devices, which showed good sensitivity as other nanostructures. To this approach, $\alpha-\mathrm{MoO}_{3}$ nanoflakes, SnS nanoflakes, and $\mathrm{Au}$ NPs incorporated $\mathrm{MoS}_{2}$ nanoflakes were developed by researchers by means of RF sputtering, solid-state reaction, and chemical exfoliation, respectively [117-119]. Wherein, SnS nanoflakes seems to be an impressive candidate with a sensor response of $>1000$ at low operating temperature $\left(100^{\circ} \mathrm{C}\right)$. Moreover, the sensor response is stable even after six weeks with a LOD of $<5 \mathrm{ppm}$ and response/recovery time of $<15 \mathrm{~s}$. Similar to the microspheres $[120,121]$, materials with nanosphere (NSP) structures are also effectively applied in the acetone estimation as noted below. Liu et al. and $\mathrm{Zhu}$ et al. developed the $\mathrm{NiO} / \mathrm{ZnO}$ and $\mathrm{WO}_{3}-\mathrm{SnO}_{2}$ hollow composite NSPs to engage in effective quantitation of acetone via solvothermal and hydrothermal methods, correspondingly [122,123]. As shown in Figure 7, solvothermally synthesized $\mathrm{NiO} / \mathrm{ZnO}$ NSPs show great responses to acetone $\left(\mathrm{R}_{\mathrm{a}} / \mathrm{R}_{\mathrm{g}}=29.8\right.$ for $100 \mathrm{ppm}$ at $\left.275^{\circ} \mathrm{C}\right)$ with an LOD down to sub-ppm level [122]. 
(i)

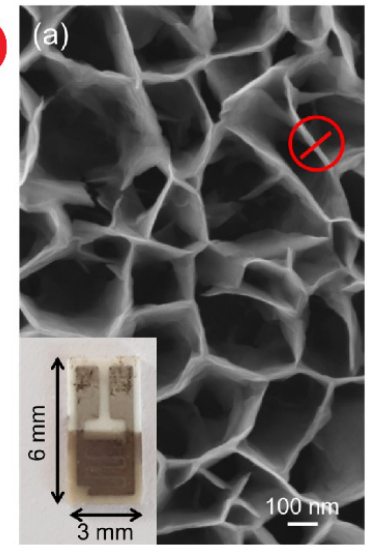

(ii)

(a)

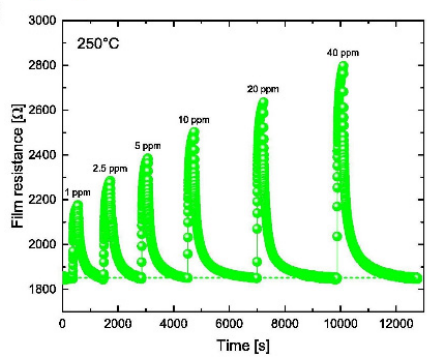

(b)
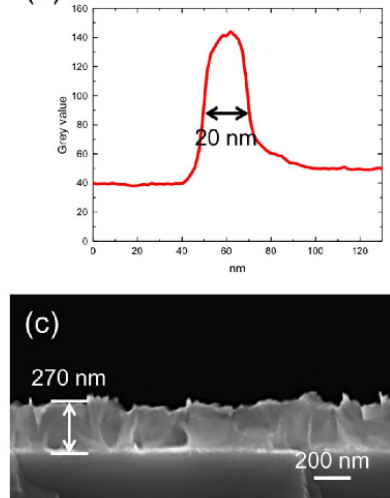

(b)

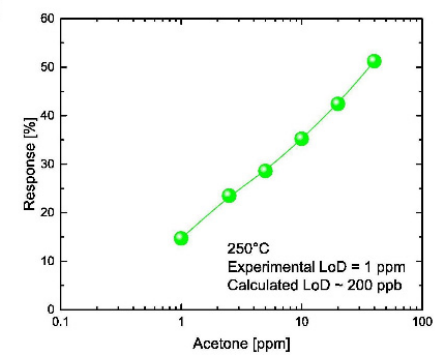

Figure 6. (i) (a) Plan-view SEM image of the NiO nanowalls (the inset shows the NiO-based sensor), (b) grey value profile along the line perpendicular to the nanosheet marked with the red circle, and (c) cross-view SEM image of the $\mathrm{NiO}$ nanowalls; (ii) (a) Dynamic responses of the NiO-based sensor to acetone pulses in the concentration range $1-40 \mathrm{ppm}$ at $250{ }^{\circ} \mathrm{C}$ and (b) calibration curve at $250{ }^{\circ} \mathrm{C}$ (Reproduced with the permission from Ref. [115]).

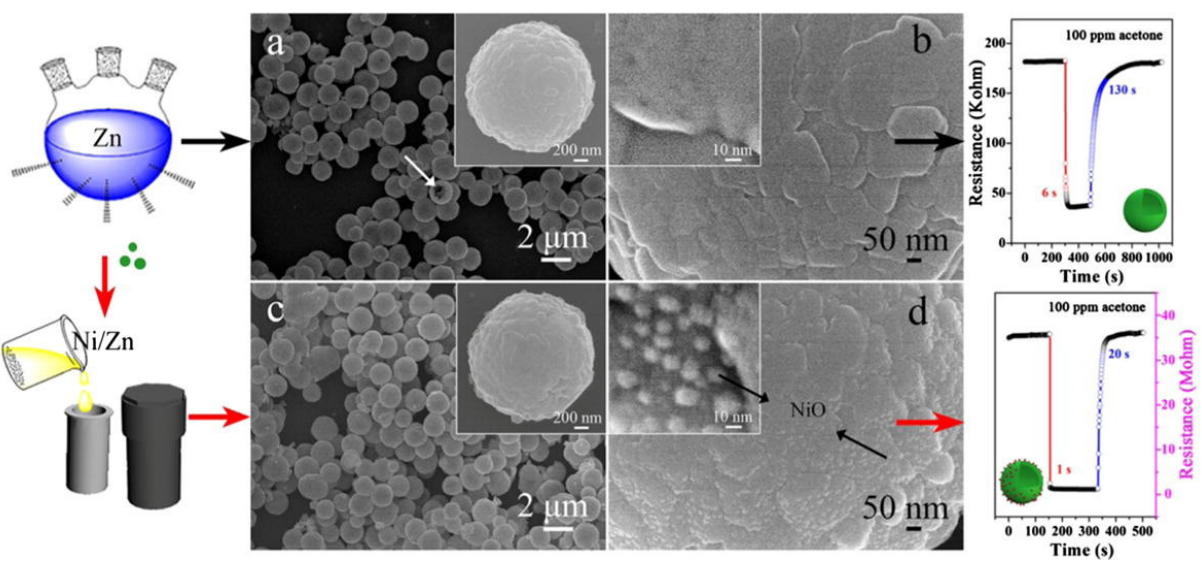

Figure 7. (a-d) Schematic representation of solvothermal synthesize, SEM images and sensory response of of $\mathrm{NiO} / \mathrm{ZnO}$ hollow spheres towards acetone (Reproduced with the permission from Ref. [122]).

In fact, the greater sensor response of $\mathrm{NiO} / \mathrm{ZnO}$ NSPs was attributed to the decoration of $\mathrm{NiO}$ nanoparticles over the $\mathrm{ZnO}$ NSPs, thus become a notable candidate in acetone detection. On the other hand, the $\mathrm{WO}_{3}-\mathrm{SnO}_{2}$ forms two kind of NSPs, namely, the $\mathrm{WO}_{3}-$ $\mathrm{SnO}_{2} 160 \mathrm{NSPs}$ and the $\mathrm{WO}_{3}-\mathrm{SnO}_{2} 190 \mathrm{NSPs}$ (prepared by heating at $160{ }^{\circ} \mathrm{C}$ and $190{ }^{\circ} \mathrm{C}$, respectively) which display good response to acetone $\left(R_{a} / R_{g}=\sim 8 \& 16\right.$ for 50 ppm at $\left.275^{\circ} \mathrm{C}\right)$. However, it still requirse further optimization to attain high linear responses and low LOD.

To this track, researchers described the acetone quantitation by flower-like structures of Na-doped p-type $\mathrm{ZnO}$, cubic-rhombohedral- $\mathrm{In}_{2} \mathrm{O}_{3}, \mathrm{Au}$ NPs functionalized $\mathrm{ZnO}$, and 
$\mathrm{RuO}_{2}$ modified $\mathrm{ZnO}$ [124-127]. In particular, the Na-doped $\mathrm{ZnO}$ nanoflowers revealed the acetone sensing under ultraviolet (UV) illumination with a LOD of $0.2 \mathrm{ppm}$ [124]. Contrast to the cubic-rhombohedral- $\operatorname{In}_{2} \mathrm{O}_{3}$ microflowers $\left(\mathrm{R}_{\mathrm{a}} / \mathrm{R}_{\mathrm{g}}=13.6\right.$ for $50 \mathrm{ppm}$ at $250{ }^{\circ} \mathrm{C}$; response $/$ recovery time $\left.=2 \mathrm{~s} / 46 \mathrm{~s} ; \mathrm{LOD}=0.01 \mathrm{ppm}\right)$ and $\mathrm{RuO}_{2}$ modified $\mathrm{ZnO}$ nanoflowers $\left(\mathrm{R}_{\mathrm{a}} / \mathrm{R}_{\mathrm{g}}=125.9\right.$ for $100 \mathrm{ppm}$ at $172{ }^{\circ} \mathrm{C}$; response $/$ recovery time $=1 \mathrm{~s} / 52 \mathrm{~s}$; LOD $\leq 25$ ppm) $[125,127]$, Wang et al. described an exceptional sensor response of Au NPs functionalized $\mathrm{ZnO}$ nanoflowers [126] as shown in Table 1. Due to the loading of Au NPs over $\mathrm{ZnO}$ surface, the response is 2900 for $100 \mathrm{ppm}$ acetone with a LOD of $<20 \mathrm{ppm}$ at higher operating temperature $\left(\sim 365^{\circ} \mathrm{C}\right)$, thereby become a notable candidate in acetone quantification. However, the working temperature requires further optimization.

Majority of nanostructures showed the importance of the pore effect on acetone detection as described in the following. Porous nanoparticles ( $\mathrm{Au}$ sensitized $\mathrm{Fe}_{2} \mathrm{O}_{3} \mathrm{NPs}$ and $\mathrm{Au} / \mathrm{ZnO} \mathrm{NPs})$, porous nanorods $\left(\mathrm{ZnFe}_{2} \mathrm{O}_{4} \mathrm{NRds}\right.$ and $\left.\alpha-\mathrm{Fe}_{2} \mathrm{O}_{3} / \mathrm{SnO}_{2} \mathrm{NRds}\right)$, porous nanospheres (Pt sensitized $\mathrm{W}_{18} \mathrm{O}_{49}$ ), nanoporous fibers $(\mathrm{ZnO} / \mathrm{C})$, porous hierarchical nanostructures ( $\mathrm{Pt}$ doped $3 \mathrm{D} \mathrm{SnO}_{2}$ and $\mathrm{Ni}$ doped $\mathrm{ZnO}$ ), and porous nanocomposites $\left(\mathrm{CuFe}{ }_{2} \mathrm{O}_{4} / \alpha-\mathrm{Fe}_{2} \mathrm{O}_{3}\right.$ and $\left(\mathrm{WO}_{3} / \mathrm{Au}\right)$ were utilized by researchers towards acetone quantification [127-137]. These nanostructures were synthesized through various hydrothermal/atomic layer deposition/template mediated tactics. As shown in Table 1, many of these porous structures revealed exceptional acetone sensing due to the factor of pore effect in the gaseous species adsorption. In particular, Pt-sensitized $\mathrm{W}_{18} \mathrm{O}_{49}$ nanospheres and Pt-doped 3D porous $\mathrm{SnO}_{2}$ hierarchical structures $[132,134]$ reported exceptional sensing of acetone $\left(\mathrm{R}_{\mathrm{a}} / \mathrm{R}_{\mathrm{g}}=85\right.$ (for $20 \mathrm{ppm}$ ) and 505.7 (for $100 \mathrm{ppm}$ ) at $180^{\circ} \mathrm{C}$ and $153{ }^{\circ} \mathrm{C}$, respectively) with the LODs down to sub-ppm level ( $\sim .05 \mathrm{ppm})$, thereby become the unique candidates. Owing to the porous effect on VOCs detection, $\mathrm{ZnO} / \mathrm{C}$ nanoporous fibers [133] evidenced the sensitivity to both acetone and ethanol $\left(R_{a} / R_{g}=53.222\right.$ and 59.273 (for $100 \mathrm{ppm}$ ) at $370{ }^{\circ} \mathrm{C}$ ) with LODs down to sub-ppm level. But further optimizations are required on the operating temperature and selectivity to particular VOC.

Other than porous nanostructures, hierarchical nanostructures synthesized by either hydrothermal or thermal oxidation tactics were effectively applied in acetone detection. Hierarchical nanostructures of $\mathrm{ZnO} N W s-l o a d e d ~ S b-d o p e d ~ \mathrm{SnO}_{2}-\mathrm{ZnO}$, 3D flower-like $\mathrm{ZnO}$, and $\mathrm{Au}$ NPs-SnO $\mathrm{S}_{2}$ NTs seems to be impressive in the selective detection of acetone at operating temperatures $\geq 200^{\circ} \mathrm{C}$ as presented in Table 1 [138-140]. Wherein, Wang et al. described the direct transformation of $\mathrm{SnS}_{2}$ NShs to hierarchical porous $\mathrm{SnO}_{2}$ NTs via thermal oxidation, which evinced a better selectivity upon Au NPs decoration at $200{ }^{\circ} \mathrm{C}$ with a LOD of $0.445 \mathrm{ppm}$, thereby stated as an exceptional candidate in acetone sensing [140]. To this light, differently shaped nanostructures were synthesized by the tactics like hydrothermal, impregnation, templated synthesize, etc. 3D inverse opal (3DIO) $\mathrm{In}_{2} \mathrm{O}_{3}$ $\mathrm{CuO}$ nano-architecture, $3 \mathrm{D}$ grass-like carbon-doped $\mathrm{ZrO}_{2}$ nano-architecture, $\mathrm{Sm}_{2} \mathrm{O}_{3}$ loaded mulberry shaped $\mathrm{SnO}_{2}$ hierarchical nanostructure, cactus-like $\mathrm{WO}_{3}-\mathrm{SnO}_{2}$ nanocomposite, walnut like architecture of $\mathrm{Fe}$ and $\mathrm{C}$ co-doped $\mathrm{WO}_{3}$, Urchin like $\mathrm{Cr}$-doped $\mathrm{WO}_{3}$ hollow nanospheres, and core-shell heterostructure of $\mathrm{ZnO} / \mathrm{MoS}_{2}$ NShs were engaged in effective detection of acetone as shown in Table 1 [141-147]. Wherein, 3D grass-like carbon-doped $\mathrm{ZrO}_{2}$ architecture film displays the selectivity to both alcohols and acetone [142], thereby cannot be stated as a better candidate for acetone detection. Similarly, as seen in Figure 8, walnut like architecture of $\mathrm{Fe}$ and $\mathrm{C}$ co-doped $\mathrm{WO}_{3}$, lying underneath microstructure rather than nanostructure, shows high selectivity to acetone $\left(R_{a} / R_{g}=\sim 18\right.$ for $\left.10 \mathrm{ppm}\right)$ at $300{ }^{\circ} \mathrm{C}$ even after 12 weeks [145]. Moreover, it can detect the acetone even at sub-ppm concentration $(\sim 0.2 \mathrm{ppm})$ and the sensor can operates at $90 \%$ humid condition, thus become an exceptional candidate in acetone quantitation. Apart from the aforementioned diverse nanostructures, nanocomposites that are not described under any unique nanoarchitectures also show their high selective sensitivity to acetone [148-153] as shown in Table 1. Wherein, a few of them operate at diverse humid conditions $(10-90 \% \mathrm{RH})$ and some others require optimization of operating temperature to attain high sensitivity. 

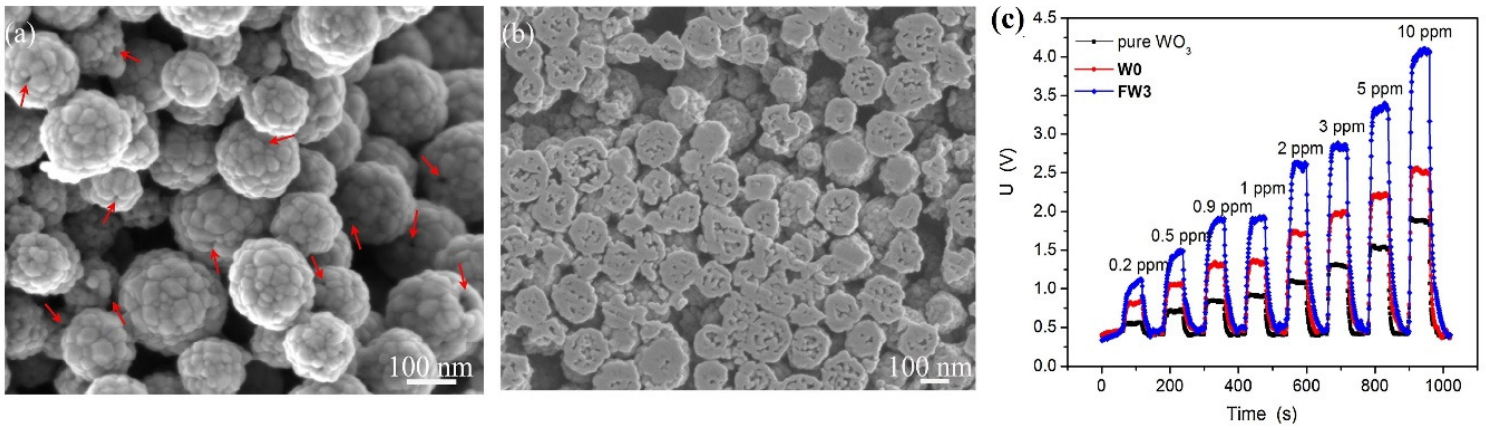

Figure 8. $(\mathbf{a}, \mathbf{b})$ Top-view and cross-sectional SEM image of $\mathrm{Fe}$ and $\mathrm{C}$ codoped $\mathrm{WO}_{3}$ (red arrow signifying the walnut like architecture); (c) Dynamic response-recovery curves of the three sensors based on the prepared pure $\mathrm{WO}_{3}, \mathrm{C}$ codoped $\mathrm{WO}_{3}$ (W0) and $\mathrm{Fe}$ and $\mathrm{C}$ codoped $\mathrm{WO}_{3}$ (FW3) sensors to different concentrations of acetone at $300{ }^{\circ} \mathrm{C}$ (Reproduced with the permission from Ref. [145]).

Table 1. Detection concentration, response/recovery time, operating temperature (Temp.) and limits of dection (LODs) to acetone gas by diverse nanostructured material.

\begin{tabular}{|c|c|c|c|c|c|c|}
\hline Materials/Nanostructure & Analyte/Concentration & $\begin{array}{l}\text { Gas Response } \\
\left(\mathrm{S}=\mathbf{R}_{\mathrm{air}} / \mathbf{R}_{\mathrm{gas}}\right)\end{array}$ & Response/Recovery & Temp. & LOD & Ref \\
\hline $\mathrm{TiO}_{2} /$ nanoparticles & Acetone/500 ppm & 9.19 & $10 \mathrm{~s} / 9 \mathrm{~s}$ & $270^{\circ} \mathrm{C}$ & $0.5 \mathrm{ppm}$ & [59] \\
\hline$\alpha-\mathrm{Fe}_{2} \mathrm{O}_{3} /$ nanoparticles & Acetone/100 ppm & 8.8 & NA & $340^{\circ} \mathrm{C}$ & $5 \mathrm{ppm}$ & [60] \\
\hline $\begin{array}{c}\text { Mn doped } \\
\mathrm{ZnO} / \text { nanoparticles }\end{array}$ & Acetone $/ 2$ ppm & 3.7 & 17 s/NA & $340^{\circ} \mathrm{C}$ & $1.8 \mathrm{ppm}$ & [61] \\
\hline $\begin{array}{l}\text { Pt-decorated Al-doped } \\
\mathrm{ZnO} / \text { nanoparticles }\end{array}$ & Acetone $/ 10 \mathrm{ppm}$ & 421 & $2.9 \mathrm{~s} / 440 \mathrm{~s}$ & $450^{\circ} \mathrm{C}$ & $\sim 0.1 \mathrm{ppm}$ & [62] \\
\hline $\begin{array}{c}\text { Al doped } \\
\mathrm{ZnO} / \text { nanoparticles }\end{array}$ & Acetone $/ 10 \mathrm{ppm}$ & 11.8 & $11 \mathrm{~s} / 793 \mathrm{~s}$ & $500^{\circ} \mathrm{C}$ & $0.01 \mathrm{ppm}$ & [63] \\
\hline B-TiO ${ }_{2} @ \mathrm{Ag} /$ nanoparticles & Acetone $/ 50 \mathrm{ppm}$ & 68.19 & $12 \mathrm{~s} / 41 \mathrm{~s}$ & $250^{\circ} \mathrm{C}$ & $0.887 \mathrm{ppm}$ & [64] \\
\hline $\mathrm{La}_{1-\mathrm{x}} \mathrm{Y}_{\mathrm{x}} \mathrm{MnO}_{3-\delta} /$ nanoparticles & Acetone/500 ppm & 27.2 & NA & $300^{\circ} \mathrm{C}$ & NA & [65] \\
\hline $\mathrm{Bi}_{1-\mathrm{x}} \mathrm{La}_{\mathrm{x}} \mathrm{FeO}_{3} /$ nanoparticles & Acetone/0.05 ppm & 8 & $15 \mathrm{~s} / 13 \mathrm{~s}$ & $260^{\circ} \mathrm{C}$ & $0.05 \mathrm{ppm}$ & [66] \\
\hline $\mathrm{SmFe}_{1-x} \mathrm{Mg}_{\mathrm{x}} \mathrm{O}_{3} /$ nanocrystals & Acetone/0.5 ppm & 7.16 & $32 \mathrm{~s} / 8 \mathrm{~s}$ & $220^{\circ} \mathrm{C}$ & $0.01 \mathrm{ppm}$ & [67] \\
\hline $\mathrm{WO}_{3 /}$ nanocrystals & Acetone $/ 0.25 \mathrm{ppm}$ & 3.8 & $4 \mathrm{~s} / 5 \mathrm{~s}$ & $320^{\circ} \mathrm{C}$ & $0.0075 \mathrm{ppm}$ & [68] \\
\hline $\mathrm{TiO}_{2}-5 \mathrm{Rh} /$ nanocrystals & Acetone/50 ppm & 9.6 & NA & $300^{\circ} \mathrm{C}$ & $10 \mathrm{ppm}$ & [69] \\
\hline $\begin{array}{c}\mathrm{Co}_{3} \mathrm{O}_{4} \mathrm{NPs} \text { attached } \\
\mathrm{SnO}_{2} / \text { nanowires }\end{array}$ & Acetone $/ 50 \mathrm{ppm}$ & 70 & $\mathrm{NA} / 122 \mathrm{~s}$ & $300^{\circ} \mathrm{C}$ & $0.5 \mathrm{ppm}$ & [70] \\
\hline $\begin{array}{c}\text { self-assembled monolayer } \\
\text { (SAM) functionalized } \\
\text { ZnO/nanowires }\end{array}$ & Acetone $/ 50 \mathrm{ppm}$ & $170 \& 90$ & $\begin{array}{l}2 \mathrm{~min} / 24 \mathrm{~min} \& \\
3 \mathrm{~min} / 29 \mathrm{~min}\end{array}$ & $300^{\circ} \mathrm{C}$ & $0.5 \mathrm{ppm}$ & [71] \\
\hline $\begin{array}{c}\text { Branched p-Cu } \mathrm{Cu}_{\mathrm{x}} \mathrm{O} @ \\
\mathrm{n}-\mathrm{ZnO} / \text { nanowires }\end{array}$ & Acetone $/ 5-50 \mathrm{ppm}$ & $3.39-6.38$ & $62 \mathrm{~s} / 90 \mathrm{~s}$ & $250^{\circ} \mathrm{C}$ & $\sim 5 \mathrm{ppm}$ & [72] \\
\hline $\begin{array}{c}\mathrm{Cr} \text { doped } \mathrm{ZnO} \\
\text { single-crystal/nanorods }\end{array}$ & Acetone/70 ppm & 70 & NA & $300^{\circ} \mathrm{C}$ & $\sim 10 \mathrm{ppm}$ & [73] \\
\hline $\mathrm{SnS}_{2} /$ nanorods & Acetone/ 10 ppm & 25 & NA & $300^{\circ} \mathrm{C}$ & $\begin{array}{l}\text { Down to } \\
\text { sub-ppm }\end{array}$ & [74] \\
\hline $\begin{array}{c}\mathrm{Au} @ \mathrm{ZnO} \& \\
\mathrm{Pd} @ \mathrm{ZnO} / \text { nanorods }\end{array}$ & Acetone/100 ppm & $44.5 \& 31.8$ & $8 \mathrm{~s} / 5 \mathrm{~s} \& 17 \mathrm{~s} / 11 \mathrm{~s}$ & $150^{\circ} \mathrm{C}$ & $0.005 \mathrm{ppm}$ & [75] \\
\hline$\alpha-\mathrm{Fe}_{2} \mathrm{O}_{3}-\mathrm{NiO} /$ nanorods & Acetone/100 ppm & 290 & $28 \mathrm{~s} / 40 \mathrm{~s}$ & $280^{\circ} \mathrm{C}$ & $\sim 5 \mathrm{ppm}$ & [76] \\
\hline $\begin{array}{c}\text { Ag-doped } \\
\mathrm{ZnO} / \text { nanoneedles }\end{array}$ & Acetone $/ 200$ ppm & 30.233 & $10 \mathrm{~s} / 21 \mathrm{~s}$ & $370^{\circ} \mathrm{C}$ & $\sim 10 \mathrm{ppm}$ & [77] \\
\hline La-doped $\mathrm{SnO}_{2} /$ nanoarrays & Acetone/200 ppm & 69 & $6-12 \mathrm{~s} / 20 \mathrm{~s}$ & $290^{\circ} \mathrm{C}$ & $5 \mathrm{ppm}$ & [78] \\
\hline$\alpha-\mathrm{Fe}_{2} \mathrm{O}_{3}-\mathrm{SnO}_{2} /$ nanoarrays & Acetone $/ 1 \mathrm{ppm}$ & 5.37 & $14 \mathrm{~s} / 70 \mathrm{~s}$ & $340^{\circ} \mathrm{C}$ & $1 \mathrm{ppm}$ & [79] \\
\hline $\mathrm{ZnTiO}_{3} /$ nanoarrays & Acetone/12.5 ppm & $78 \& 94$ & $\begin{array}{l}117 \text { and } 141 \mathrm{~s} / 99 \text { and } \\
131 \mathrm{~s} \& 75 \text { and } 81 \mathrm{~s} / 50 \\
\text { and } 69 \mathrm{~s} \text { (dark \& light) }\end{array}$ & $45^{\circ} \mathrm{C} \& 350^{\circ} \mathrm{C}$ & $\begin{array}{l}0.01 \& 0.09 \\
\quad \text { ppm }\end{array}$ & [80] \\
\hline
\end{tabular}


Table 1. Cont.

\begin{tabular}{|c|c|c|c|c|c|c|}
\hline Materials/Nanostructure & Analyte/Concentration & $\begin{array}{l}\text { Gas Response } \\
\left(\mathrm{S}=\mathrm{R}_{\mathrm{air}} / \mathbf{R}_{\mathrm{gas}}\right)\end{array}$ & Response/Recovery & Temp. & LOD & Ref \\
\hline $\begin{array}{c}\text { Ag-decorated } \\
\mathrm{SnO}_{2} / \text { nanofibers }\end{array}$ & Acetone $/ 50 \mathrm{ppm}$ & 40 & $6 \mathrm{~s} / 10 \mathrm{~s}$ & $160^{\circ} \mathrm{C}$ & $5 \mathrm{ppm}$ & [81] \\
\hline $\mathrm{PrFeO}_{3 / \text { nanofibers }}$ & Acetone/200 ppm & 141.3 & $7 \mathrm{~s} / 6 \mathrm{~s}$ & $180^{\circ} \mathrm{C}$ & $10 \mathrm{ppm}$ & [82] \\
\hline Pt-ZnO- $\mathrm{In}_{2} \mathrm{O}_{3} /$ nanofibers & Acetone/100 ppm & 57.1 & $1 \mathrm{~s} / 44 \mathrm{~s}$ & $300^{\circ} \mathrm{C}$ & $0.5 \mathrm{ppm}$ & [83] \\
\hline $\mathrm{Au} @ \mathrm{WO}_{3}-\mathrm{SnO}_{2} /$ nanofibers & Acetone/10 ppm & 196.1 & $\sim 2 \min$ (for both) & $150^{\circ} \mathrm{C}$ & $<0.5 \mathrm{ppm}$ & [84] \\
\hline $\begin{array}{l}\text { Au functionalized In-doped } \\
\mathrm{ZnSnO}_{3} / \text { nanofibers }\end{array}$ & Acetone $/ 50 \mathrm{ppm}$ & 19.3 & $10 \mathrm{~s} / 13 \mathrm{~s}$ & $200^{\circ} \mathrm{C}$ & $\sim 10 \mathrm{ppm}$ & [85] \\
\hline $\mathrm{ZnO} /$ nanofibers & Acetone/100 ppm & $50-124$ & $65-130 \mathrm{~s} / 75-135 \mathrm{~s}$ & $250^{\circ} \mathrm{C}$ & NA & [86] \\
\hline $\mathrm{Ru}$-doped $\mathrm{SnO}_{2}$, nanofibers & Acetone/100 ppm & 118.8 & $1 \mathrm{~s} / 86 \mathrm{~s}$ & $200^{\circ} \mathrm{C}$ & $\sim 0.5 \mathrm{ppm}$ & [87] \\
\hline $\mathrm{Pd}-\mathrm{SnO}_{2} /$ nanotubes & Acetone $/ 5$ ppm & 93.55 & NA & $350^{\circ} \mathrm{C}$ & $<1 \mathrm{ppm}$ & [88] \\
\hline $\begin{array}{c}\mathrm{PdO} @ \mathrm{ZnO}- \\
\mathrm{SnO}_{2} / \text { nanotubes }\end{array}$ & Acetone $/ 1$ ppm & 5.06 & $20 \mathrm{~s} / 64 \mathrm{~s}$ & $400^{\circ} \mathrm{C}$ & $0.01 \mathrm{ppm}$ & [89] \\
\hline $\begin{array}{c}\alpha-\mathrm{Fe}_{2} \mathrm{O}_{3} \text { nanorods- } \\
\text { MWCNTs/nanotubes }\end{array}$ & Acetone/100 ppm & 38.7 & $2 \mathrm{~s} / 45 \mathrm{~s}$ & $225^{\circ} \mathrm{C}$ & $0.5 \mathrm{ppm}$ & [90] \\
\hline $\begin{array}{c}\text { Pt-decorated } \\
\mathrm{CuFe}_{2} \mathrm{O}_{4} / \text { nanotubes }\end{array}$ & Acetone/100 ppm & 16.5 & $16 \mathrm{~s} / 299 \mathrm{~s}$ & $300^{\circ} \mathrm{C}$ & $\sim 5 \mathrm{ppm}$ & [92] \\
\hline $\mathrm{WO}_{3}-\mathrm{SnO}_{2} /$ nanotubes & Acetone $/ 50 \mathrm{ppm}$ & 63.8 & NA & $275^{\circ} \mathrm{C}$ & $0.05 \mathrm{ppm}$ & [93] \\
\hline $\begin{array}{l}\text { ZnO-Decorated In/Ga } \\
\text { Oxide/nanotubes }\end{array}$ & Acetone/100 ppm & $12.7 \& 27.1$ & $\begin{array}{l}6.8 \mathrm{~s} / 6.1 \mathrm{~s} \& 11.8 \\
\mathrm{~s} / 11.6 \mathrm{~s}\end{array}$ & $300^{\circ} \mathrm{C}$ & $0.2 \mathrm{ppm}$ & [94] \\
\hline $\mathrm{Y}$ doped $\mathrm{SnO}_{2} /$ nanobelts & Acetone/100 ppm & 11.4 & $9-25 \mathrm{~s} / 10-30 \mathrm{~s}$ & $210^{\circ} \mathrm{C}$ & $0.9024 \mathrm{ppm}$ & [95] \\
\hline Eu doped $\mathrm{SnO}_{2} /$ nanobelts & Acetone/100 ppm & 8.56 & $15 \mathrm{~s} / 19 \mathrm{~s}$ & $210^{\circ} \mathrm{C}$ & $0.131 \mathrm{ppm}$ & [96] \\
\hline $\mathrm{Co}_{3} \mathrm{O}_{4 / \text { nanocubes }}$ & Acetone/500 ppm & 4.88 & $2 \mathrm{~s} / 5 \mathrm{~s}$ & $240^{\circ} \mathrm{C}$ & $\sim 10 \mathrm{ppm}$ & [97] \\
\hline $\mathrm{Ag}-\mathrm{ZnSnO} /$ /nanocubes & Acetone $/ 100 \mathrm{ppm}$ & 30 & $2 \mathrm{~s} / 3 \mathrm{~s}$ & $280^{\circ} \mathrm{C}$ & $1 \mathrm{ppm}$ & [99] \\
\hline $\mathrm{ZnO}-\mathrm{CuO} /$ nanocubes & Acetone $/ 1 \mathrm{ppm}$ & 11.14 & NA & $200^{\circ} \mathrm{C}$ & $0.009 \mathrm{ppm}$ & [100] \\
\hline $\mathrm{NiFe}_{2} \mathrm{O}_{4} /$ nanocubes & Acetone/1 ppm & 1.9 & $8 \mathrm{~s} / 40 \mathrm{~s}$ & $160^{\circ} \mathrm{C}$ & $0.52 \mathrm{ppm}$ & [101] \\
\hline $\mathrm{NiO} / \mathrm{ZnO} /$ nanocubes & Acetone/200 ppm & 58 & $24 \mathrm{~s} / 133 \mathrm{~s}$ & $340^{\circ} \mathrm{C}$ & $\sim 10 \mathrm{ppm}$ & [102] \\
\hline $\begin{array}{c}\text { MOF derived- } \\
\mathrm{ZnO} / \mathrm{ZnFe}_{2} \mathrm{O}_{4} / \text { nanocubes }\end{array}$ & Acetone $/ 5$ ppm & 9.4 & $5.6 \mathrm{~min} / 6 \mathrm{~min}$ & $250^{\circ} \mathrm{C}$ & $<0.5 \mathrm{ppm}$ & [103] \\
\hline $\mathrm{PdO} @ \mathrm{Co}_{3} \mathrm{O}_{4} /$ nanocages & Acetone $/ 5 \mathrm{ppm}$ & 2.51 & NA & $350^{\circ} \mathrm{C}$ & $0.1 \mathrm{ppm}$ & [104] \\
\hline $\mathrm{ZnO} / \mathrm{ZnFe}_{2} \mathrm{O}_{4} /$ nanocages & Acetone/100 ppm & 25.8 & $8 \mathrm{~s} / 32 \mathrm{~s}$ & $290^{\circ} \mathrm{C}$ & $1 \mathrm{ppm}$ & [105] \\
\hline $\begin{array}{c}\mathrm{PdO}- \\
\mathrm{NiO} / \mathrm{NiCO}_{2} \mathrm{O}_{4} / \text { nanocages }\end{array}$ & Acetone/100 ppm & 6.7 & $<20 \mathrm{~s} /<30 \mathrm{~s}$ & $210^{\circ} \mathrm{C}$ & NA & [106] \\
\hline Ag@CuO- $\mathrm{TiO}_{2} /$ nanocages & Acetone/100 ppm & 6.2 & $56 \mathrm{~s} / 9 \mathrm{~s}$ & $200^{\circ} \mathrm{C}$ & $\sim 1 \mathrm{ppm}$ & [107] \\
\hline $\mathrm{Co}_{3} \mathrm{O}_{4} /$ nanosheets & Acetone/100 ppm & 16.5 & NA & $111^{\circ} \mathrm{C}$ & $\sim 5 \mathrm{ppm}$ & [108] \\
\hline $\mathrm{ZnO} /$ nanosheets & Acetone $/ 5$ ppm & 6.7 & $<60 \mathrm{~s}$ (for both) & $300^{\circ} \mathrm{C}$ & $<5 \mathrm{ppm}$ & [109] \\
\hline $\mathrm{SnO}_{2} / \mathrm{Fe}_{2} \mathrm{O}_{3} /$ nanosheets & Acetone/10 ppm & 9.8 & $0.8 \mathrm{~s} / 3.4 \mathrm{~s}$ & $260^{\circ} \mathrm{C}$ & NA & [110] \\
\hline $\mathrm{NiO} /$ nanosheets & Acetone/150 ppm & $>90$ & $80 \mathrm{~s} / 82 \mathrm{~s}$ & $200^{\circ} \mathrm{C}$ & $0.83 \mathrm{ppm}$ & [111] \\
\hline $\begin{array}{l}\text { Fluorine doped } \\
\mathrm{TiO}_{2} / \text { nanosheets }\end{array}$ & Acetone/800 ppm & 17.42 & $162 \mathrm{~s} / 220.5 \mathrm{~s}$ & $25^{\circ} \mathrm{C}$ & $\sim 25 \mathrm{ppm}$ & [112] \\
\hline $\begin{array}{l}\mathrm{Nb} \text {-doped } \mathrm{ZnO} \& \\
\mathrm{ZnO} / \text { nanowalls }\end{array}$ & Acetone/100 ppm & $84.62 \& 89.13$ & NA & $200^{\circ} \mathrm{C} \& 200^{\circ} \mathrm{C}$ & $<20 \mathrm{ppm}$ & [113] \\
\hline $\mathrm{CuO} /$ nanowalls & Acetone/500 ppm & 4 & $82 \mathrm{~s}(50 \mathrm{ppm}) / \mathrm{NA}$ & $320^{\circ} \mathrm{C}$ & $2 \mathrm{ppm}$ & [114] \\
\hline $\mathrm{NiO} /$ nanowalls & Acetone/10 ppm & $>30$ & NA & $250^{\circ} \mathrm{C}$ & $0.2 \mathrm{ppm}$ & [115] \\
\hline$\alpha-\mathrm{MoO}_{3} /$ nanoflakes & Acetone/10-100 ppm & NA & NA & $150^{\circ} \mathrm{C}$ & NA & [117] \\
\hline SnS/nanoflakes & Acetone/100 ppm & $>1000$ & $3 \mathrm{~s} / 14 \mathrm{~s}$ & $100^{\circ} \mathrm{C}$ & $<5$ ppm & [118] \\
\hline $\mathrm{NiO} / \mathrm{ZnO} /$ nanospheres & Acetone/100 ppm & 29.8 & $1 \mathrm{~s} / 20 \mathrm{~s}$ & $275^{\circ} \mathrm{C}$ & $\begin{array}{l}\text { Down to } \\
\text { sub-ppm }\end{array}$ & [122] \\
\hline $\mathrm{WO}_{3}-\mathrm{SnO}_{2} /$ nanospheres & Acetone $/ 50 \mathrm{ppm}$ & $\sim 8 \& 16$ & $16 \mathrm{~s} / 12 \mathrm{~s} \& 15 \mathrm{~s} / 11 \mathrm{~s}$ & $240^{\circ} \mathrm{C}$ & $\sim 50 \mathrm{ppm}$ & [123] \\
\hline
\end{tabular}


Table 1. Cont.

\begin{tabular}{|c|c|c|c|c|c|c|}
\hline Materials/Nanostructure & Analyte/Concentration & $\begin{array}{l}\text { Gas Response } \\
\left(\mathrm{S}=\mathrm{R}_{\text {air }} / \mathrm{R}_{\mathrm{gas}}\right)\end{array}$ & Response/Recovery & Temp. & LOD & Ref \\
\hline $\mathrm{Na}: \mathrm{ZnO} /$ nanoflowers & Acetone/100 ppm & 3.35 & $18.2 \mathrm{~s} / 63 \mathrm{~s}$ & NA & $0.2 \mathrm{ppm}$ & {$[124]$} \\
\hline $\mathrm{ZnO} /$ nanoflowers & Acetone/100 ppm & $2900 \& 300$ & $5 \mathrm{~s} / \mathrm{NA}$ & $365^{\circ} \mathrm{C} \& 248^{\circ} \mathrm{C}$ & $<20$ ppm & [126] \\
\hline $\begin{array}{c}\mathrm{RuO}_{2} \text {-modified } \\
\mathrm{ZnO} / \text { nanoflowers }\end{array}$ & Acetone/100 ppm & 125.9 & $1 \mathrm{~s} / 52 \mathrm{~s}$ & $172{ }^{\circ} \mathrm{C}$ & $<25$ ppm & {$[127]$} \\
\hline $\begin{array}{c}\mathrm{Au} \mathrm{NPs}-\mathrm{Fe}_{2} \mathrm{O}_{3} / \text { porous } \\
\text { nanoparticles }\end{array}$ & Acetone/10 ppm & 6.1 & $5 \mathrm{~s} / 20 \mathrm{~s}$ & $200^{\circ} \mathrm{C}$ & $0.132 \mathrm{ppm}$ & {$[128]$} \\
\hline $\begin{array}{l}\mathrm{Au} / \mathrm{ZnO} / \text { porous } \\
\text { nanoparticles }\end{array}$ & Acetone/1 ppm & 17.1 & $231 \mathrm{~s} / 215 \mathrm{~s}$ & $275^{\circ} \mathrm{C}$ & $<0.1 \mathrm{ppm}$ & {$[129]$} \\
\hline $\mathrm{ZnFe}_{2} \mathrm{O}_{4} /$ porous nanorods & Acetone/100 ppm & 52.8 & $1 \mathrm{~s} / 11 \mathrm{~s}$ & $260^{\circ} \mathrm{C}$ & $<10$ ppm & {$[130]$} \\
\hline $\begin{array}{c}\alpha-\mathrm{Fe}_{2} \mathrm{O}_{3} / \mathrm{SnO}_{2} / \text { porous } \\
\text { nanorods }\end{array}$ & Acetone/100 ppm & 53.1 & $9 \mathrm{~s} / 2.5 \mathrm{~s}$ & $260^{\circ} \mathrm{C}$ & $<10$ ppm & {$[131]$} \\
\hline $\begin{array}{c}\mathrm{W}_{18} \mathrm{O}_{49} / \mathrm{Pt} / \text { porous } \\
\text { nanospheres }\end{array}$ & Acetone/20 ppm & 85 & $13 \mathrm{~s} / 11 \mathrm{~s}(50 \mathrm{ppm})$ & $180^{\circ} \mathrm{C}$ & $0.052 \mathrm{ppm}$ & {$[132]$} \\
\hline $\begin{array}{l}\text { Pt-doped-3D-SnO } \mathrm{S}_{2} / \text { porous } \\
\text { hierarchical structure }\end{array}$ & Acetone/100 ppm & 505.7 & $130 \mathrm{~s} / 140 \mathrm{~s}$ & $153^{\circ} \mathrm{C}$ & $<0.05$ ppm & {$[134]$} \\
\hline $\begin{array}{l}\text { Ni doped } \mathrm{ZnO} / \text { porous } \\
\text { hierarchical structure }\end{array}$ & Acetone/100 ppm & 68 & $6 \mathrm{~s} / 2 \mathrm{~s}$ & $340^{\circ} \mathrm{C}$ & 0.116 ppm & [135] \\
\hline $\begin{array}{c}\mathrm{CuFe}_{2} \mathrm{O}_{4} / \alpha-\mathrm{Fe}_{2} \mathrm{O}_{3} / \text { porous } \\
\text { composite shell }\end{array}$ & Acetone/100 ppm & 14 & NA & $275^{\circ} \mathrm{C}$ & $0.1 \mathrm{ppm}$ & [136] \\
\hline $\begin{array}{l}\text { 3D- } \mathrm{WO}_{3} / \mathrm{Au} / \text { porous nano- } \\
\text { composite }\end{array}$ & Acetone/1.5 ppm & 7.6 & $7 \mathrm{~s} / 8 \mathrm{~s}$ & $410^{\circ} \mathrm{C}$ & $0.1 \mathrm{ppm}$ & [137] \\
\hline $\begin{array}{l}\mathrm{ZnO} \text { nanowires-loaded } \\
\text { Sb-doped } \\
\mathrm{SnO}_{2}-\mathrm{ZnO} / \text { hierarchical } \\
\text { structure }\end{array}$ & Acetone/5 ppm & $12.1 \& 27.8$ & $\begin{array}{c}<16 \text { s \& } 32 \text { s (res)/NA } \\
\text { (rec) }\end{array}$ & $400^{\circ} \mathrm{C}$ & $4.3 \& 8.1 \mathrm{ppm}$ & [138] \\
\hline $\begin{array}{l}\mathrm{ZnO} / 3 \mathrm{D}-\text { flower-like } \\
\text { hierarchical structure }\end{array}$ & Acetone/100 ppm & 18.6 & $7 \mathrm{~s} / \mathrm{NA}$ & $300^{\circ} \mathrm{C}$ & NA & [139] \\
\hline $\begin{array}{c}\mathrm{Au}-\mathrm{SnO}_{2} / \text { hierarchical } \\
\text { structure }\end{array}$ & Acetone/100 ppm & 40.42 & $22 \mathrm{~s} / 90 \mathrm{~s}$ & $200^{\circ} \mathrm{C}$ & 0.445 ppm & [140] \\
\hline $\begin{array}{c}\mathrm{In}_{2} \mathrm{O}_{3}-\mathrm{CuO} / 3 \mathrm{D} \text {-inverse } \\
\text { opals structure }\end{array}$ & Acetone/0.5 ppm & 4.8 & $13 \mathrm{~s} / 20 \mathrm{~s}$ & $370^{\circ} \mathrm{C}$ & 0.05 ppm & [141] \\
\hline $\begin{array}{c}\mathrm{SnO}_{2} / \mathrm{Sm}_{2} \mathrm{O}_{3} / \text { mulberry- } \\
\text { shaped } \\
\text { structure }\end{array}$ & Acetone/100 ppm & 41.14 & NA & $250^{\circ} \mathrm{C}$ & $0.1 \mathrm{ppm}$ & [143] \\
\hline $\begin{array}{c}\mathrm{WO}_{3}-\mathrm{SnO}_{2} / \text { cactus like } \\
\text { nano-composite }\end{array}$ & Acetone/600 ppm & 26 & NA & $360^{\circ} \mathrm{C}$ & NA & {$[144]$} \\
\hline $\begin{array}{c}\mathrm{Cr} \text { doped } \mathrm{WO}_{3} / \text { urchin-like } \\
\text { hollowspheres }\end{array}$ & Acetone/10 ppm & 13.3 & NA & $250^{\circ} \mathrm{C}$ & $0.467 \mathrm{ppm}$ & [146] \\
\hline $\begin{array}{c}\mathrm{ZnO} / \mathrm{MoS} 2 \\
\text { nanosheets/core-shell } \\
\text { nanostructure }\end{array}$ & $\begin{array}{l}\text { Acetone / } 5 \text { ppm \& } 20 \\
\text { ppm (No UV and UV) }\end{array}$ & $14.4 \& 4.67$ & $71 s / 35 s \& 56 s / 69 s$ & $300^{\circ} \mathrm{C} \& 100{ }^{\circ} \mathrm{C}$ & $0.1 \mathrm{ppm}$ & [147] \\
\hline $\begin{array}{l}\text { RGO-h- } \mathrm{WO}_{3} / \text { nano- } \\
\text { composite }\end{array}$ & Acetone/ 200 ppm & 1.5 & $14 \mathrm{~s} / \mathrm{NA}$ & Room Temp. & $<1$ ppm & [148] \\
\hline $\begin{array}{c}2 \mathrm{D}-\mathrm{C}_{3} \mathrm{~N}_{4}-\mathrm{SnO}_{2} / \text { nano- } \\
\text { composite }\end{array}$ & Acetone/100 ppm & 29 & $10 \mathrm{~s} / 11 \mathrm{~s}$ & $380^{\circ} \mathrm{C}$ & $0.067 \mathrm{ppm}$ & [149] \\
\hline $\begin{array}{c}\text { In loaded } \mathrm{WO}_{3} / \mathrm{SnO}_{2} / \text { nano- } \\
\text { composite }\end{array}$ & Acetone/50 ppm & 66.5 & $11 \mathrm{~s} / 5.5 \mathrm{~s}$ & $200^{\circ} \mathrm{C}$ & $<1 \mathrm{ppm}$ & {$[150]$} \\
\hline $\begin{array}{l}\mathrm{Co}_{3} \mathrm{O}_{4} \text { nanowires-hollow } \\
\text { carbon } \\
\text { spheres/nano-composite }\end{array}$ & Acetone/200 ppm & 23 & NA & $200{ }^{\circ} \mathrm{C}$ & $<1$ ppm & [151] \\
\hline $\begin{array}{c}\mathrm{Fe}_{2} \mathrm{O}_{3} / \mathrm{In}_{2} \mathrm{O}_{3} / \text { nano- } \\
\text { composite }\end{array}$ & Acetone/100 ppm & $>15$ & $8 \mathrm{~s} / 6 \mathrm{~s}$ & $200^{\circ} \mathrm{C}$ & NA & [152] \\
\hline $\begin{array}{l}\mathrm{CuO}-\mathrm{Ga}_{2} \mathrm{O}_{3} / \text { nano- } \\
\text { composite }\end{array}$ & Acetone/1.25 ppm & $\sim 1.3$ & NA & $300^{\circ} \mathrm{C}$ & $0.1 \mathrm{ppm}$ & [153] \\
\hline
\end{tabular}

NA = Not available; Temp. = Temperature; $\mathrm{s}=$ seconds; $\mathrm{min}=$ minutes. 


\section{Alcoholic Vapor Detection by Miscellaneous Nanostructures}

In regard to the development of alcoholic vapor detection, various nanostructured materials were placed in the gaseous chamber to interact with the adsorbed oxygen species follow the reactions described in Equations (3) and (4) to release electrons.

$$
\begin{aligned}
\left(\mathrm{C}_{\mathrm{n}} \mathrm{H}_{2 \mathrm{n}+1} \mathrm{OH}\right)_{\text {gas }} & \rightarrow\left(\mathrm{C}_{\mathrm{n}} \mathrm{H}_{2 \mathrm{n}+1} \mathrm{OH}\right)_{\text {ads }} \\
\left(\mathrm{C}_{\mathrm{n}} \mathrm{H}_{2 \mathrm{n}+1} \mathrm{OH}\right)_{\mathrm{ads}}+3 \mathrm{nO}^{-} & \rightarrow \mathrm{nCO}_{2}+(\mathrm{n}+1) \mathrm{H}_{2} \mathrm{O}+3 \mathrm{ne}^{-}
\end{aligned}
$$

Similar to the above detection process, metal oxide can oxidize the alcoholic vapor to aldehyde and convert them to water and carbon-dioxide to produce electrons as described in Equations (5)-(7).

$$
\begin{aligned}
& \left(\mathrm{C}_{n} \mathrm{H}_{2 n+1} \mathrm{OH}\right)_{\mathrm{ads}} \rightarrow\left(\mathrm{C}_{n} \mathrm{H}_{2 \mathrm{n}+1} \mathrm{O}^{-}\right)_{\mathrm{ads}}+\mathrm{H}^{+} \\
& \left(\mathrm{C}_{\mathrm{n}} \mathrm{H}_{2 \mathrm{n}+1} \mathrm{O}^{-}\right)_{\mathrm{ads}}+\mathrm{H}^{+} \rightarrow\left(\mathrm{C}_{\mathrm{n}} \mathrm{H}_{2 \mathrm{n}+1} \mathrm{O}\right)_{\text {ads }}+\mathrm{H}_{2} \\
& \left(\mathrm{C}_{\mathrm{n}} \mathrm{H}_{2 \mathrm{n}+1} \mathrm{O}\right)_{\mathrm{ads}}+(3 \mathrm{n}-1) \mathrm{O}_{\text {ads }}^{-} \rightarrow \mathrm{nCO}_{2}+(\mathrm{n}+1) \mathrm{H}_{2} \mathrm{O}+3(\mathrm{n}-1) \mathrm{e}^{-}
\end{aligned}
$$

In the above two sensory processes, the released electrons lead to changes in resistance, which is then used as a sensor signal $\left(\mathrm{R}_{\mathrm{a}} / \mathrm{R}_{\mathrm{g}}\right)$. To this track, numerous reports with diverse nanostructures have been reported towards the sensing of alcoholic gases as detailed as following.

Through the synthetic tactics like soft-chemical approach, hydrothermal, calcination, solvothermal, and sol-gel methods, researchers proposed several nanoparticles syntheses and applications in the alcoholic vapors sensing [154-158]. $\mathrm{Sn}_{3} \mathrm{~N}_{4} \mathrm{NPs}$, Ni-doped $\mathrm{SnO}_{2}$ $\mathrm{NPs}$, C-doped $\mathrm{TiO}_{2} \mathrm{NPs}$, Pr-doped $\mathrm{In}_{2} \mathrm{O}_{3} \mathrm{NPs}$, and $\mathrm{Au} / \mathrm{Cl}$ co-modified $\mathrm{LaFeO}_{3} \mathrm{NPs}$ were engaged in the detection of alcoholic vapors. In particular, $\mathrm{Sn}_{3} \mathrm{~N}_{4} \mathrm{NPs}$ and $\mathrm{Au}$ and $\mathrm{Cl}$ co-modified $\mathrm{LaFeO}_{3} \mathrm{NPs}[154,158]$ displayed good sensor responses to ethanol vapor with decent response/recovery time at an optimum operating temperature of $120^{\circ} \mathrm{C}$ as shown in Table 2. Whereas, C-doped $\mathrm{TiO}_{2} \mathrm{NPs}$ were demonstrated in the detection of n-pentanol at $170{ }^{\circ} \mathrm{C}$ with a LOD down to sub-ppm level [156]. In this light, Ni-doped $\mathrm{SnO}_{2} \mathrm{NPs}$ were noted as an exceptional candidate, which showed sensitivity to both n-butanol and formaldehyde via tuning the doping concentrations of Ni ions [155]. Two percent Nidoped $\mathrm{SnO}_{2}$ NPs displayed a remarkable sensor response $\left(\mathrm{R}_{\mathrm{a}} / \mathrm{R}_{\mathrm{g}}=1690.7\right)$ to $\mathrm{n}$-butanol at $160{ }^{\circ} \mathrm{C}$ with a response/recovery time of $10 \mathrm{~s} />10 \mathrm{~min}$. Moreover, $4 \% \mathrm{Ni}$-doped $\mathrm{SnO}_{2} \mathrm{NPs}$ demonstrated a sensor response $\left(\mathrm{R}_{\mathrm{a}} / \mathrm{R}_{\mathrm{g}}=1298\right)$ at $100{ }^{\circ} \mathrm{C}$ with the response/recovery time of $6 \mathrm{~s} />10 \mathrm{~min}$. Their detection of $\mathrm{n}$-butanol displayed a linear response from 1 to $100 \mathrm{ppm}$ with a LOD of $\sim 1 \mathrm{ppm}$, thereby can be considered for future development.

Similar to the NPs, nanocrystalline materials were also used in the sensing applications of alcohols. Cao and co-workers explored the ethanol sensing utilities of unmodified/Clmodified $\mathrm{LaFe}_{\mathrm{x}} \mathrm{O}_{3-\delta}$ nanocrystals $[159,160]$. Both $\mathrm{LaFe}_{\mathrm{x}} \mathrm{O}_{3-\delta}$ and $\mathrm{Cl}$-modified $\mathrm{LaFe}_{\mathrm{x}} \mathrm{O}_{3-\delta}$ nanocrystals displayed sensor responses of 132 and 79.2 (for 1000 and 200 ppm, correspondingly) with the response/recovery time of $<10 \mathrm{~s}$ at $140^{\circ} \mathrm{C}$ and $136^{\circ} \mathrm{C}$, respectively. However, the proposed LODs of both nanocrystals require further optimization. Ethanol sensing was also delivered by $\alpha-\mathrm{MoO}_{3}$ and copper oxide $\left(\mathrm{CuO} / \mathrm{Cu}_{2} \mathrm{O}\right)$ nanocrystals $[161,162]$, but their operating temperatures seems to be $>250{ }^{\circ} \mathrm{C}$ (see Table 2), thus requires more interrogations. Xiaofeng et al. described the methanol detection by $\mathrm{Gd}_{1-\mathrm{x}} \mathrm{Ca}_{\mathrm{x}} \mathrm{FeO}_{3}(\mathrm{x}=0-0.4)$ nanocrystalline powder [163]. This material showed a sensor response of $R_{a} / R_{g}=117.7$ (for $600 \mathrm{ppm}$ ) at $260^{\circ} \mathrm{C}$ with a response/recovery time of $<2 \mathrm{~min}$ and a LOD of $<50 \mathrm{ppm}$. However, it still requires investigations on more interfering gaseous.

Towards the detection of ethanol, doped/functionalized nanowires, such as Au modified $\mathrm{ZnO}$ NWs, $\mathrm{Fe}_{2} \mathrm{O}_{3}$ NPs coated $\mathrm{SnO}_{2}$ NWs, $\mathrm{In}_{2} \mathrm{O}_{3}$ NPs decorated $\mathrm{ZnS} \mathrm{NWs}$, and Sr-doped cubic $\operatorname{In}_{2} \mathrm{O}_{3}$ /rhombohedral $\operatorname{In}_{2} \mathrm{O}_{3}$ homojunction, NWs displayed extensive sensitivity [164-167]. These NWs were synthesized by vapor-liquid-solid (VLS) method, hydrothermal or electrospun tactics. All NWs can operate at $300^{\circ} \mathrm{C}$ except the Au modified 
$\mathrm{ZnO}$ NWs, which operate at $350{ }^{\circ} \mathrm{C}$. In particular, Sr-doped cubic $\mathrm{In}_{2} \mathrm{O}_{3} /$ rhombohedral $\mathrm{In}_{2} \mathrm{O}_{3}$ homojunction NWs [167] are impressive in terms of the response/recovery time ( $<1 \mathrm{~min})$ with sub-ppm LOD (0.025 ppm). Similar to the NWs, nanorods are also utilized in the quantitation of ethanol as follows. $\mathrm{Cr}_{2} \mathrm{O}_{3} \mathrm{NPs}$ functionalized $\mathrm{WO}_{3} \mathrm{NRds}$, $\mathrm{ZnO}$ NRds, $\mathrm{Pd}$ NPs decorated $\mathrm{ZnO}$ NRds, and $\mathrm{SnO}_{2}-\mathrm{ZnO}$ heterostructure NRds were synthesized by thermal evaporation, hydrothermal, and chemical vapor deposition (CVD) methods and employed in ethanol sensing [168-172]. Shankar et al. reported the fabrication of three kinds of polyvinyl alcohol (PVA)-ZnO NRds calcined composites (NR1, NR2, and NR3) and demonstrated their exceptional sensitivity to ethanol at room temperature as shown in Figure 9 and Table 2 [169]. In this work, NR3 displays a good sensing performance $\left(\mathrm{R}_{\mathrm{a}} / \mathrm{R}_{\mathrm{g}}=23\right.$; response $/$ recovery time $\left.=26 \mathrm{~s} / 43 \mathrm{~s} ; \mathrm{LOD} \leq 5 \mathrm{ppm}\right)$. In view of this, $\mathrm{SnO}_{2}$ $\mathrm{ZnO}$ heterostructure NRds (operating temperature is $275^{\circ} \mathrm{C}$ ) seems to be another good candidate with the LOD of $1 \mathrm{ppm}$, but it still requires optimization to reduce the operation temperature.
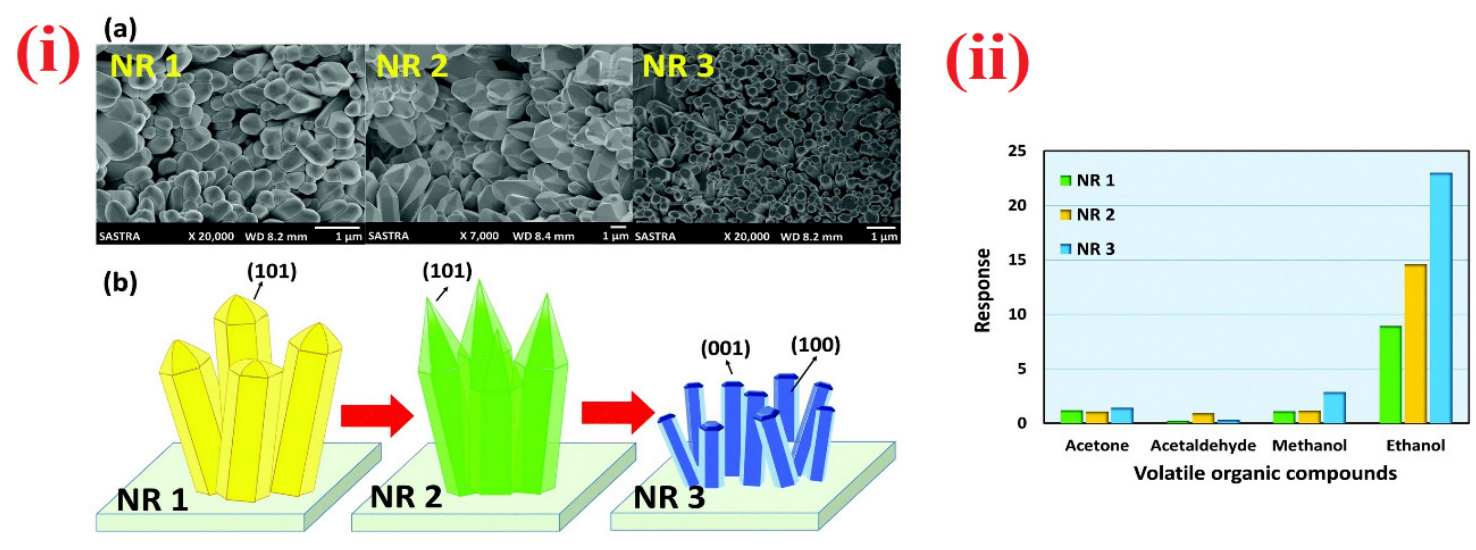

Figure 9. (i) (a) FESEM images of PVA-ZnO nanocomposites calcined at $873 \mathrm{~K}$ for different time durations-1 h (NR1), $3 \mathrm{~h}$ (NR2), and $6 \mathrm{~h}$ (NR3) and (b) schematic of the ZnO nanorods (NR1, NR2, and NR3); (ii) Sensing responses of the calcined samples towards 100 ppm of acetone, acetaldehyde, methanol, and ethanol at room temperature (299 K) (Reproduced with the permission from Ref. [169]).

On the other end of the spectrum, Perfecto et al. reported the discrimination of Iso-propyl alcohol (IPA) by rGO- $\mathrm{WO}_{3} \cdot 0.33 \mathrm{H}_{2} \mathrm{O}$ nanoneedles (rGO: reduced graphene oxide) [173]. This material was synthesized by the combination of ultrasonic spray nozzle (USN) and microwave-assisted hydrothermal (MAH) methods and displayed a sensor response of 4.96 to $100 \mathrm{ppm} \mathrm{IPA} \mathrm{at} \mathrm{room} \mathrm{temperature} \mathrm{and} \mathrm{at} \mathrm{55 \%} \mathrm{RH} \mathrm{with} \mathrm{a} \mathrm{LOD} \mathrm{of} 1 \mathrm{ppm}$. Therefore, it becomes an impressive candidate in IPA sensing. However, more investigations are required to improve the sensitivity. $\mathrm{Sm}$-doped $\mathrm{SnO}_{2}$ nanoarrays were developed through a hydrothermal tactic and applied in IPA sensing by Zhao and co-workers [174]. In which, it showed a high sensing response to IPA $\left(R_{a} / R_{g}=117.7\right.$; response $/$ recovery time $=$ $12 \mathrm{~s} / 20 \mathrm{~s}$; at $252{ }^{\circ} \mathrm{C}$ ) with a LOD of $\sim 1 \mathrm{ppm}$, therby become a notable material. However, this work requires more efforts in the reduction of operating temperature.

With regard to different alcoholic vapors detections, materials with nanofibers structures were proposed by many research groups. For example, Han et al. developed the rough $\mathrm{SmFeO}_{3}$ nanofibers via electrospinning and calcination processes and used in the determination of ethylene glycol [175]. The sensor response reached 18.19 to $100 \mathrm{ppm}$ of ethylene glycol at $240{ }^{\circ} \mathrm{C}$ with a LOD of $\sim 5 \mathrm{ppm}$. Moreover, both response/recovery time were less than a min, thus become a prominent material in ethylene glycol sensor. Similar to the above report, Feng and co-workers synthesized the In-doped NiO nanofibers through electrospinning method and employed in the detection of methanol [176]. At $300{ }^{\circ} \mathrm{C}$, the sensor response of In-doped $\mathrm{NiO}$ nanofibers reached 10.9 for $200 \mathrm{ppm}$ methanol (response/recovery time $=273 \mathrm{~s} / 26 \mathrm{~s}$ ), which was five times higher than that of pure $\mathrm{NiO}$ nanofibers. However, this work needs further optimization to minimize the work- 
ing temperature and LOD $(25 \mathrm{ppm})$. In view of this, nanofibrous materials $\left(\mathrm{SiO}_{2} @ \mathrm{SnO}_{2}\right.$ core-shell nanofibers and $\mathrm{Yb}$ doped $\mathrm{In}_{2} \mathrm{O}_{3}$ nanofibers) were also engaged in the sensing of ethanol $[177,178]$. $\mathrm{SiO}_{2} @ \mathrm{SnO}_{2}$ core-shell nanofibers [177] were synthesized by electrospinning and calcination process, but details on the sensor studies seems to be deficient (see Table 2) and need more interrogations. In contrast, $\mathrm{Yb}$-doped $\operatorname{In}_{2} \mathrm{O}_{3}$ nanofibers (developed by electrospinning) is an impressive candidate with the capability of operation at room temperature and a sensor response of 40 for $10 \mathrm{ppm}$ ethanol with a LOD of $1 \mathrm{ppm}$ [178].

Next, nanotubes were employed in the alcoholic vapor detection by using changes in resistance as the sensor signal. For example, Alali and co-workers developed the p-p heterojunction $\mathrm{CuO} / \mathrm{CuCo}_{2} \mathrm{O}_{4}$ NTs through electrospinning and applied in the sensing of n-propanol at room temperature as shown in Figure 10 [179]. The importance of this work lies on its sensor signal to $10 \mathrm{ppm}$ of $n$-propanol $\left(\mathrm{R}_{\mathrm{a}} / \mathrm{R}_{\mathrm{g}}=14\right.$; response/recovery time $=6.3 \mathrm{~s} / 4.1 \mathrm{~s}$ ). Similar to the above work, $\mathrm{p}-\mathrm{p}$ type $\mathrm{CuO}-\mathrm{NiO}$ heterojunction NTs were synthesized via calcination treatment and utilized in the detection of glycol at $110^{\circ} \mathrm{C}$ [180]. Herein, the sensor responses reached 10.35 for $100 \mathrm{ppm}$ glycol with a response/recovery time of $15 \mathrm{~s} / 45 \mathrm{~s}$ and a LOD down to sub-ppm level (0.078 ppm), thus become a remarkable material. Ethanol sensing was demonstrated by coated and doped materials with nanotubes structures ( $\mathrm{NiO}$ decorated $\mathrm{SnO}_{2}$ NTs, Ca-doped $\mathrm{In}_{2} \mathrm{O}_{3}$ NTs, Ni-doped $\mathrm{In}_{2} \mathrm{O}_{3}$ NTs, and $\mathrm{W}$-doped NiO NTs) at diverse operating temperatures [181-184]. These nanotubes were synthesized by hydrothermal or electrospinning tactics and successfully used in the discrimination of ethanol. The $\mathrm{NiO}$ decorated $\mathrm{SnO}_{2} \mathrm{NTs}$ [181] showed ethanol sensing ability of vertical standing NTs at $250{ }^{\circ} \mathrm{C}$, but was low in sensitivity and LOD $\left(\mathrm{R}_{\mathrm{a}} / \mathrm{R}_{\mathrm{g}}=123.7\right.$ for $1000 \mathrm{ppm}$; response/recovery time $=10 \mathrm{~s} / 58 \mathrm{~s}$ ). On the other hand, enhanced sensor responses were observed in the rest of the doped NTs at $\geq 160{ }^{\circ} \mathrm{C}$ with a LODs of $\sim 5 \mathrm{ppm}$. For example, Ca-doped $\mathrm{In}_{2} \mathrm{O}_{3}$ NTs [182] reached a highest sensor response of 183.3 for $100 \mathrm{ppm}$ ethanol (response/recovery time $=2 \mathrm{~s} / 56 \mathrm{~s}$ ) with a LOD of $<5 \mathrm{ppm}$ at $240{ }^{\circ} \mathrm{C}$, thus, is noted as a good material for ethanol sensors.

Discrimination of ethanol vapor was demonstrated by materials with nanobelt structures as discussed in the following. $\mathrm{In}_{2} \mathrm{O}_{3} \mathrm{NPs}$ deposited $\mathrm{TiO}_{2}$ nanobelts, $\alpha-\mathrm{MoO}_{3}$ nanobelts, and $\mathrm{Zn}$-doped $\mathrm{MoO}_{3}$ nanobelts were hydrothermally prepared and engaged in ethanol detection at $100{ }^{\circ} \mathrm{C}, 300{ }^{\circ} \mathrm{C}$, and $240{ }^{\circ} \mathrm{C}$, respectively [185-187]. Among them, $\mathrm{In}_{2} \mathrm{O}_{3}$ NPs deposited $\mathrm{TiO}_{2}$ nanobelts [185] seems to be an impressive candidate with a sensor response of $>9$ for $100 \mathrm{ppm}$ ethanol, working temperature at $100{ }^{\circ} \mathrm{C}$, response/recovery time of $6 \mathrm{~s} / 3 \mathrm{~s}$ ), and a LOD of 1 ppm as noted in Table 2. To this track, Wang et al. described the n-butanol sensing utility of nanocube structured $\mathrm{Fe}_{2} \mathrm{O}_{3}$ that was derived from MOF via calcination [188]. Upon the exposure to 100 ppm n-buatnol, the MOF derived $\mathrm{Fe}_{2} \mathrm{O}_{3}$ nanocubes displayed a sensor response of $\sim 6$ (for $100 \mathrm{ppm}$ ) at $160-230^{\circ} \mathrm{C}$, response/recovery time is $<2$ min with a LOD of $<1 \mathrm{ppm}$. Similarly, Nguyen et al. delivered the ethanol sensor property of hydrothermally synthesized $\mathrm{In}_{2} \mathrm{O}_{3}$ nanocubes [189]. In which, the sensor response reached 85 at $300{ }^{\circ} \mathrm{C}$ for $100 \mathrm{ppm}$ ethanol with lower response/recovery time (15 s/60 s) and a LOD of $<5 \mathrm{ppm}$, thus is noted as a noteworthy material in ethanol detection. However, further optimization is required in reducing the working temperature.

Other than the microcages [190], nanocages were also reported in ethanol quantitation by the researchers. ZIF-8 derived $\mathrm{ZnO}$ hollow nanocages, $\mathrm{ZIF}-8$ derived-Ag-functionalized $\mathrm{ZnO}$ hollow nanocages, and $\mathrm{Cu}_{2} \mathrm{O}$ hollow dodecahedral nanocages were employed in the exceptional detection of ethanol [191-193]. Among them, both ZIF-8 derived ZnO hollow nanocages and ZIF-8 derived-Ag-functionalized $\mathrm{ZnO}$ hollow nanocages were reported with high sensor responses to $100 \mathrm{ppm}$ ethanol $\left(\mathrm{R}_{\mathrm{a}} / \mathrm{R}_{\mathrm{g}}=139.41\right.$; response $/$ recovery time $=$ $2.8 \mathrm{~s} / 56.4 \mathrm{~s}$ at $325^{\circ} \mathrm{C}$ and $\mathrm{R}_{\mathrm{a}} / \mathrm{R}_{\mathrm{g}}=84.6$; response/recovery time $=5 \mathrm{~s} / 10 \mathrm{~s}$ at $275^{\circ} \mathrm{C}$, correspondingly) with their LODs down to sub-ppm levels (0.025 and $0.0231 \mathrm{ppm}$, respectively). In the light of this, such materials developments are appreciated with further interrogations to reduce the working temperature. Nanosheet structured materials were also developed for the quantification of gaseous ethanol. Wherein, Al-doped ultrathin $\mathrm{ZnO}$ NShs, $\mathrm{NiO}$ NPs decorated $\mathrm{SnO}_{2}$ NShs, and $\mathrm{CuO}$ NPs decorated ultrathin $\mathrm{ZnO}$ NShs displayed their 
sensor responses to ethanol vapor [194-196]. However, apart from sensor responses and LODs, these materials require optimization for working temperatures, which are $>250{ }^{\circ} \mathrm{C}$, as noted in Table 2 .
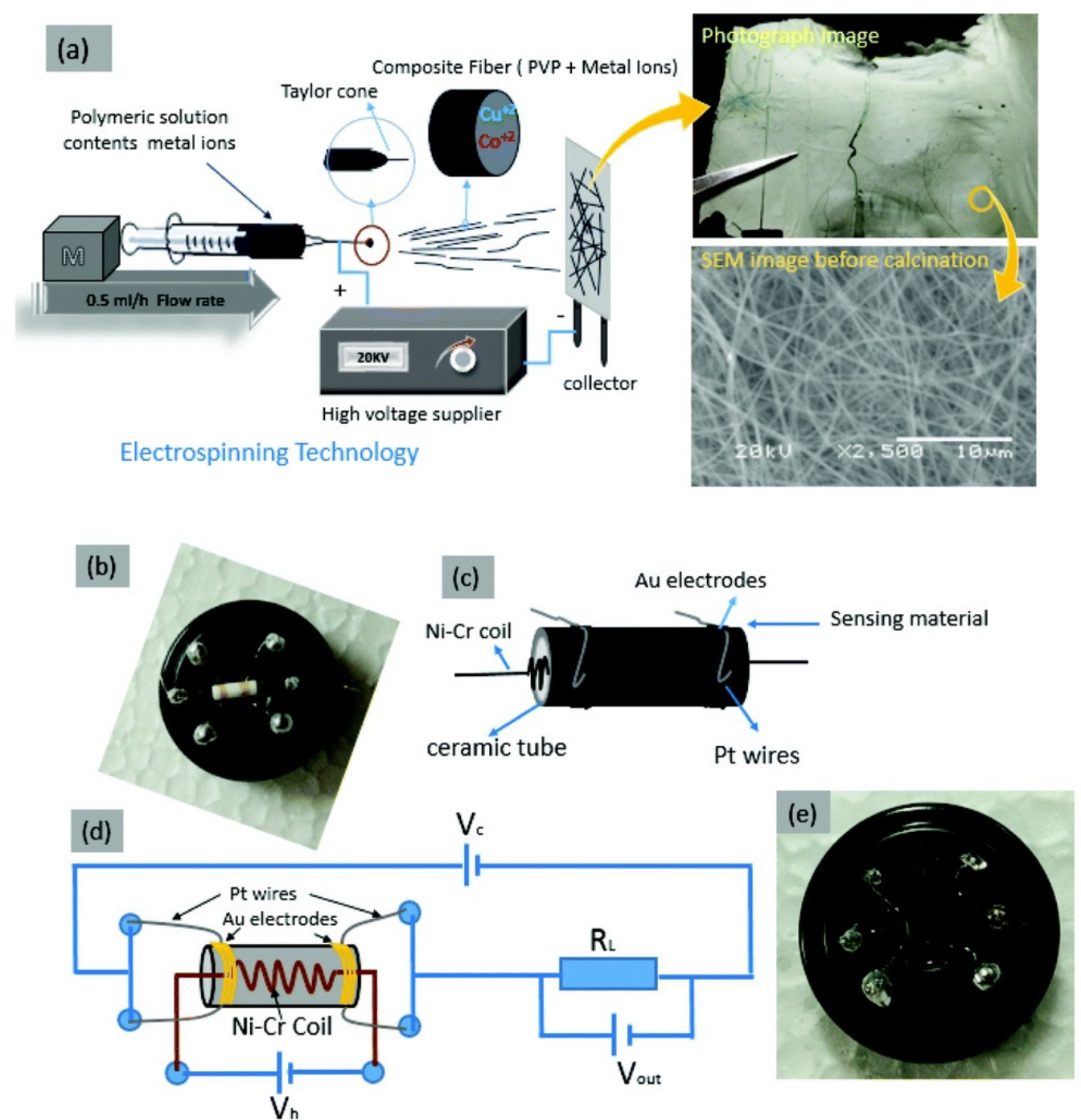

Figure 10. (a) A diagram of the electrospinning process with a photograph of as-spun fiber mat and SEM image of composite fibers before calcination treatment. (b,e) Photographs of the sensor without and with covering by sensing materials, respectively. (c) Schematic diagram of the sensor and its components. (d) A schematic of the sensor circuit and its elements (Reproduced with the permission from Ref. [179]).

In addition to diverse nanostructure-based alcoholic vapor detection, $\mathrm{SnS}_{2}$ and $\mathrm{CdS}$ nanoflakes were proposed for the sensing of methanol and IPA [197,198]. Bharatula and co-workers identified the methanol sensing performance of $\mathrm{SnS}_{2}$ nanoflakes [197]. As illustrated in Figure 11, the $\mathrm{SnS}_{2}$ nanoflakes exhibit an exceptional sensor response of 1580 for 150 ppm gas exposure at room temperature with the response/recovery time of $67 \mathrm{~s} / 5 \mathrm{~s}$, thereby can be commercialized towards the detection of methanol. However, more investigations on interference studies are required. In view of this, Liu et al. demonstrated the IPA sensing properties of CdS nanoflakes [198] with a LOD down to sub-ppm level (0.05 ppm). However, the sensor studies of CdS nanoflakes require high temperature $\left(275^{\circ} \mathrm{C}\right)$ and also lack interference studies. 
(i)

(a)
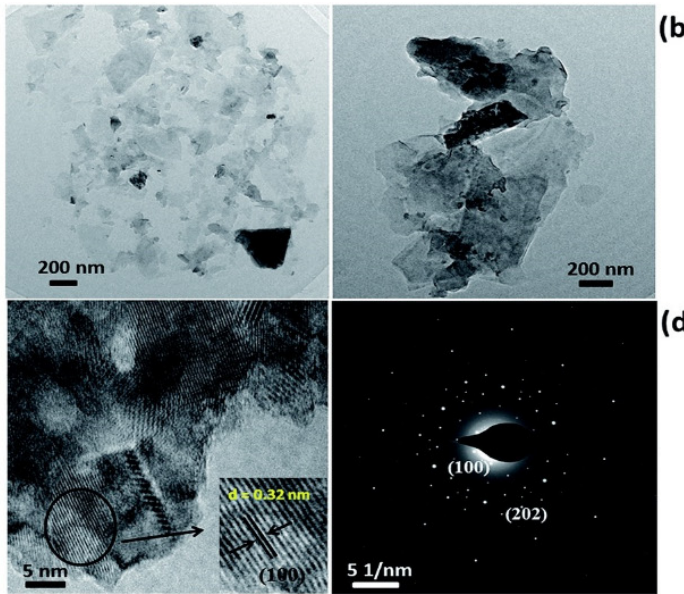

(b)

(c)

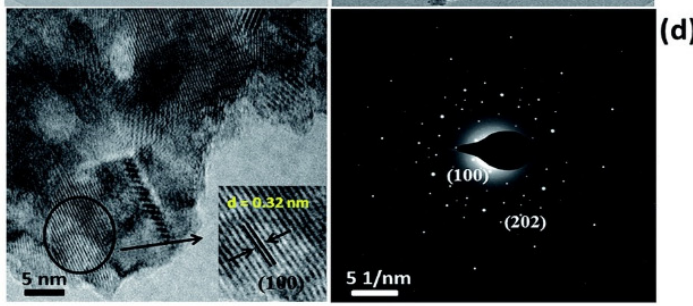

(ii)

(a)

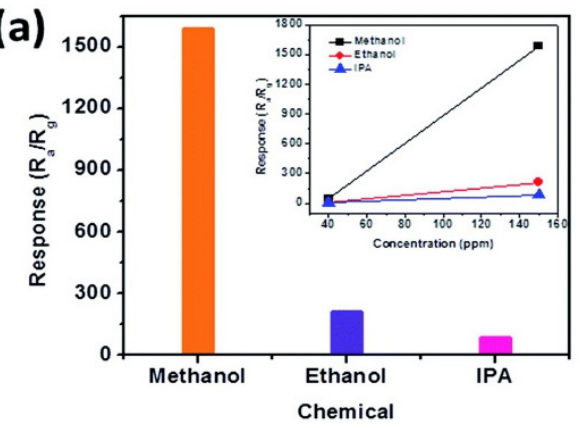

(c)

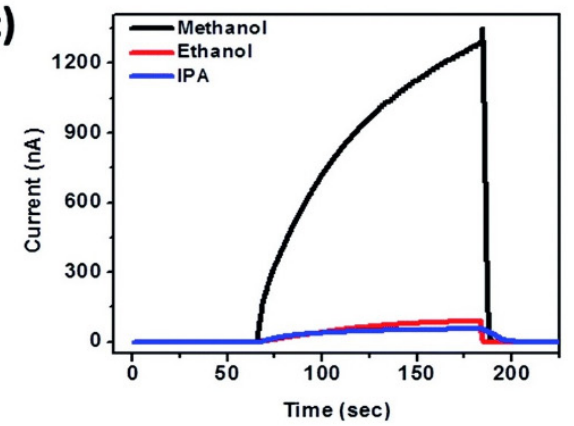

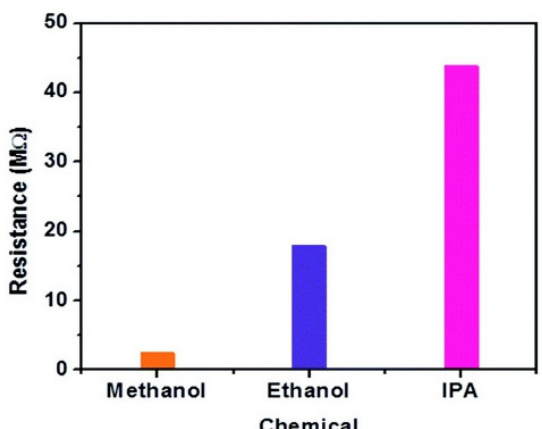

(b)

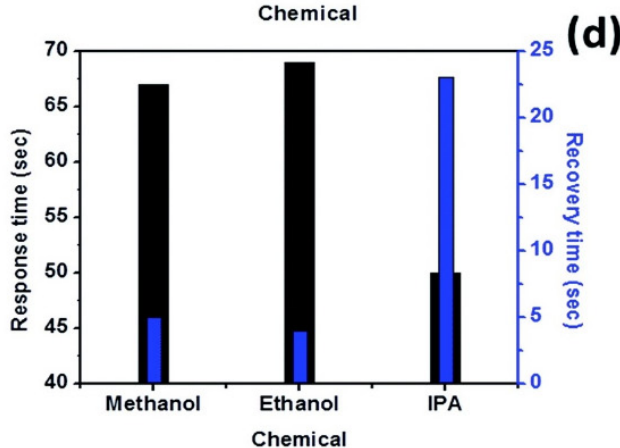

Figure 11. (i) TEM analysis of $\mathrm{SnS}_{2}$ nanoflakes (a and b) low magnification TEM images, (c) high resolution TEM image and (d) selected area diffraction (SAED) pattern; (ii) Alcohol sensing performance of $\mathrm{SnS}_{2}$ nanoflakes (all alcohols at $150 \mathrm{ppm}$ ) at $25{ }^{\circ} \mathrm{C}$ (a) response vs. alcohols and inset shows the response vs. concentration, (b) resistance vs. alcohols, (c) typical $I-t$ plot and (d) bar diagram of response and recovery time of the test alcohols (Reproduced with the permission from Ref. [197]).

Materials such as Co-doped $\mathrm{ZnO}$ hexagonal nanoplates, $\mathrm{ZIF}-8$ derived $\alpha-\mathrm{Fe}_{2} \mathrm{O}_{3} / \mathrm{ZnO} /$ $\mathrm{Au}$ hexagonal nanoplates, and $\mathrm{ZnO}$ nanoplates were reported in the ethanol detection [199-201]. The sensor responses of those nanoplates were found as 570, 170, and 8.5 for 300,100 , and $1000 \mathrm{ppm}$ of ethanol at $300^{\circ} \mathrm{C}, 280^{\circ} \mathrm{C}$, and $164^{\circ} \mathrm{C}$, correspondingly. Wherein, hydrothermally synthesized $\mathrm{Co}$-doped $\mathrm{ZnO}$ hexagonal nanoplates displayed a high sensor response to both ethanol and acetone [199]. However, further works on the interference studies as well as optimization for working temperature are required. On the other hand, compared to $\mathrm{ZnO}$ nanoplates [201], samples of ZIF-8 derived $\alpha-\mathrm{Fe}_{2} \mathrm{O}_{3} / \mathrm{ZnO} / \mathrm{Au}$ hexagonal nanoplates (synthesized by multi-step reaction process) [200] seems to be impressive in terms of response/recovery time ( $5 \mathrm{~s} / 4 \mathrm{~s})$ and LOD ( 10 ppm) as shown in Figure 12. Materials with NSPs structures towards alcoholic gas detection were proposed by the researchers. 
(i)
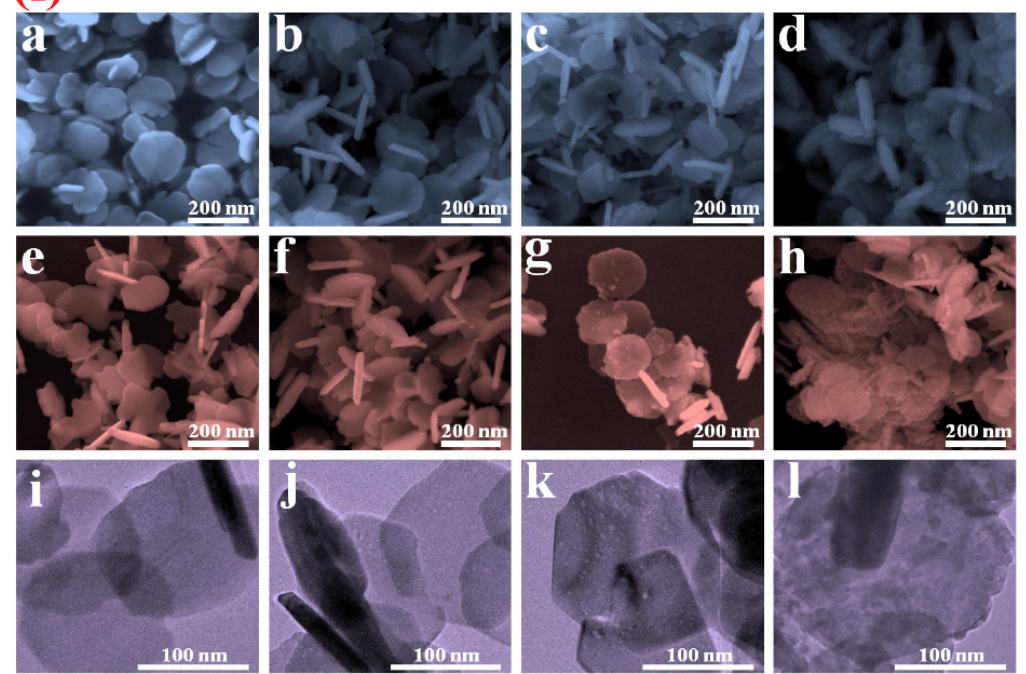

(ii)
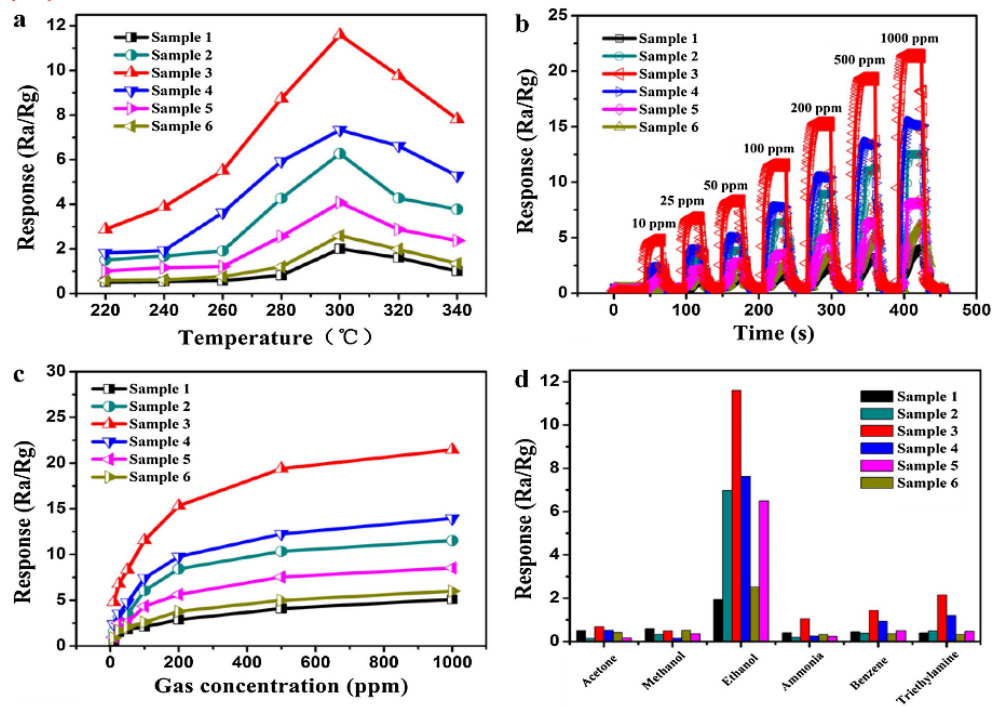

Figure 12. (i) SEM images of Sample 1-4: (a-d) before calcination; (e-h) after calcination; TEM images of Sample 1-4 after calcination (i-1); (ii) (a) The response of samples to $100 \mathrm{ppm}$ ethanol at different operating temperature; (b) the response and recovery curves of samples upon exposure to 10-1000 ppm ethanol; (c) the response curves of samples to ethanol concentrations; (d) the linear relationship of $\log (\mathrm{S}-1)-\log (\mathrm{C})$ plot to ethanol at the optimum operating temperatures (Reproduced with the permission from Ref. [200]).

Nanosphere-shaped materials such as $\mathrm{Zn}_{2} \mathrm{SnO}_{4}$, monodispersed indium tungsten oxide, $\mathrm{Ag} @ \mathrm{In}_{2} \mathrm{O}_{3}, \mathrm{ZnSnO}{ }_{3}, \mathrm{ZnO}$, and $\alpha-\mathrm{Fe}_{2} \mathrm{O}_{3}$ were effectively applied in the discrimination of alcoholic vapors as presented in Table 2 [202-207]. Wherein, $\mathrm{Zn}_{2} \mathrm{SnO}_{4} \mathrm{NSPs}$, and $\mathrm{Ag} @ \mathrm{In}_{2} \mathrm{O}_{3}$ core-shell NSPs [202,204] were found to be effective in detecting the ethanol with sensor response of 23.4 and 72.56 (for $50 \mathrm{ppm}$ ethanol at $180^{\circ} \mathrm{C}$ and $220^{\circ} \mathrm{C}$, individually) with response/recovery time of $<1 \mathrm{~min}$ and LODs of $\sim 5 \mathrm{ppm}$ and $\sim 2 \mathrm{ppm}$, correspondingly. Similarly, monodispersed indium tungsten oxide ellipsoidal NSPs and $\alpha-\mathrm{Fe}_{2} \mathrm{O}_{3}$ hollow NSPs $[203,207]$ were engaged in the quantitation of methanol at higher working temperature $\left(>250{ }^{\circ} \mathrm{C}\right)$. However, $\alpha-\mathrm{Fe}_{2} \mathrm{O}_{3}$ hollow NSPs were found to be more impressive with a sensor response of 25 for $10 \mathrm{ppm}$ methanol at $280^{\circ} \mathrm{C}$ (response/recovery time $=8 \mathrm{~s} / 9 \mathrm{~s}$ ) and with a LOD of $1 \mathrm{ppm}$. Subsequently, perovskite type $\mathrm{ZnSnO}_{3}$ NSPs and $\mathrm{ZnO}$ hollow NSPs $[205,206]$ were applied in the detection of n-propanol and n-butanol (for 500 ppm 
at $200{ }^{\circ} \mathrm{C}$ and $385{ }^{\circ} \mathrm{C}$, respectively). Between them, $\mathrm{ZnSnO}_{3} \mathrm{NSPs}$ seems to be a better candidate with respect to their working temperature and LOD (0.5 ppm).

In light of this, materials with modified nanoflower structures (PdO NPs modified $\mathrm{ZnO}, \mathrm{rGO}$ nanosheets modified $\mathrm{NiCo}_{2} \mathrm{~S}_{4}$ and $\mathrm{Pd}$ and $\mathrm{rGO}$ modified $\mathrm{TiO}_{2}$ ) and grained nanoflowers (NiO) were utilized in alcoholic gases assays [208-211]. PdO NPs modified $\mathrm{ZnO}$ nanoflowers displayed their enhanced sensing capability of methanol via decoration of PdO NPs over the surface of $\mathrm{ZnO}$ as shown in Figure 13. In a similar fashion, reduced graphene oxide ( $\mathrm{rGO}$ ) nanosheets modified $\mathrm{NiCo}_{2} \mathrm{~S}_{4}$ nanoflowers and $\mathrm{Pd}$ and rGO modified $\mathrm{TiO}_{2}$ nanoflowers were demonstrated in the detection of ethanol as noted in Table 2.
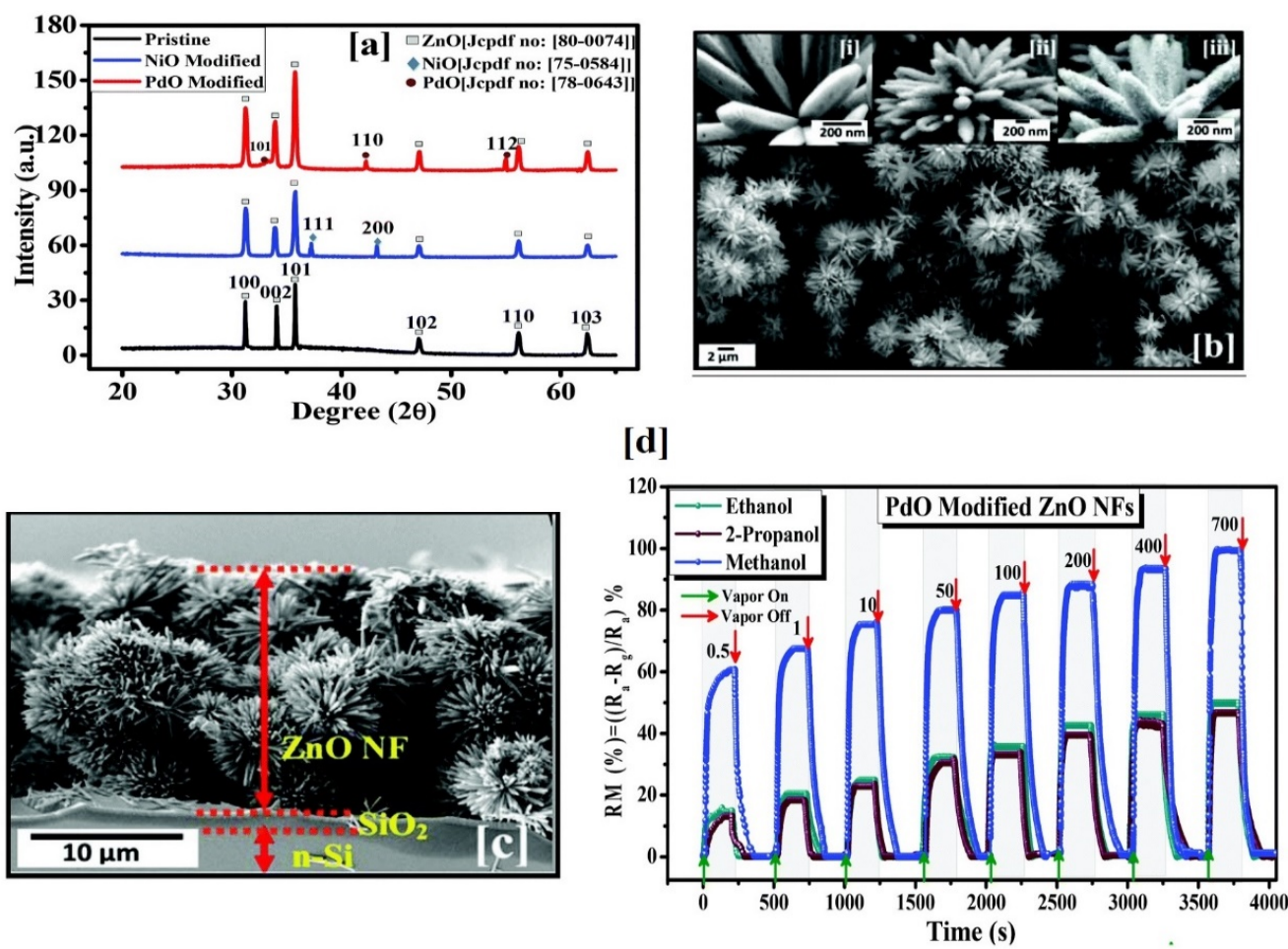

Figure 13. (a) X-ray diffraction patterns of the synthesized pristine ZnO NFs, NiO modified ZnO NFs, and PdO modified ZnO NFs. (b) FESEM image of pristine ZnO NF; inset shows magnified view of (i) pristine, (ii) NiO modified and (iii) PdO modified $\mathrm{ZnO}$ NF. (c) Cross-sectional view of grown $\mathrm{ZnO} N F$ on $\mathrm{Si} / \mathrm{SiO}_{2}$ substrate. (d) Transient response characteristics (response magnitude (\%) as a function of time) of the PdO-ZnO NF hybrid structure towards methanol, ethanol, and 2-propanol in the concentration range of 0.5-700 ppm at $150{ }^{\circ} \mathrm{C}$ (Reproduced with the permission from Ref. [208]).

In particular, $\mathrm{rGO}$ modified nanoflowers were highly impressive in terms of the operating temperature $\left(\leq 100{ }^{\circ} \mathrm{C}\right)$ and can be commercialized in future. Nanomaterials with porosity plays a vital role in the sensing studies of volatile alcoholic compounds. Porous structures of Ag-functionalized $\mathrm{ZnO}, \mathrm{Al}$-doped $\mathrm{ZnO}$, Au loaded $\mathrm{WO}_{3}$, 3D-ordered In-doped ZnO, Si@ZnO NPs, Ag loaded graphitic $\mathrm{C}_{3} \mathrm{~N}_{4}$, hierarchical mixed Pd $/ \mathrm{SnO}_{2}, \mathrm{SnO}_{2}$ fibers, hierarchical branched $\mathrm{TiO}_{2}-\mathrm{SnO}_{2}$, and hierarchical Co-doped $\mathrm{ZnO}$ were reported for their alcohol sensing utilities [212-221]. These porous nanostructures were synthesized by combustion method, nanocasting method, template mediated synthesis, microemulsion method, microdispensing method, solvothermal method, and calcination tactics. As noted in Table 2, these meso-/macro-porous nanostructures displays their good responses to alcoholic vapors at different working temperatures (lie between 150 and $350{ }^{\circ} \mathrm{C}$ ). For example, hierarchical Co-doped $\mathrm{ZnO}$ mesoporous structure [221] revealed its exceptional sensitivity to ethanol $\left(\mathrm{R}_{\mathrm{a}} / \mathrm{R}_{\mathrm{g}}=54\right.$ for $50 \mathrm{ppm}$ at $180^{\circ} \mathrm{C}$; response/recovery time $\left.=22 \mathrm{~s} / 53 \mathrm{~s}\right)$ with a LOD of $0.0454 \mathrm{ppm}$, thereby can be attested as a good candidate for ethanol sensing studies. 
Next, hierarchical nanostructures/nanocomposites were reported towards the detection of alcoholic gases. For instance, hierarchical $\mathrm{Fe}_{2} \mathrm{O}_{3}$ NRds on $\mathrm{SnO}_{2} \mathrm{NSPs}$ nanocomposites and $\mathrm{MoO}_{3}$-mixed $\mathrm{SnO}_{2}$ hierarchical aerogel nanostructures were employed in the quantification of ethanol at $320^{\circ} \mathrm{C}$ and $260{ }^{\circ} \mathrm{C}$, respectively [222,223]. The sensors responses of these materials are 23.512 and 714 , correspondingly, with response/recovery time $<1 \mathrm{~min} / 7 \mathrm{~min}$, as shown in Table 2. In a similar trend, hierarchical $\operatorname{In}_{2} \mathrm{O}_{3}$ NPs decorated $\mathrm{ZnO}$ nanostructure [224] displayed discrimination of $n$-butanol $\left(\mathrm{R}_{\mathrm{a}} / \mathrm{R}_{\mathrm{g}}=218.3\right.$ for $100 \mathrm{ppm}$ at $260^{\circ} \mathrm{C}$; response $/$ recovery time $=22 \mathrm{~s} / 53 \mathrm{~s}$ ) with a LOD down to sub-ppm level, thereby become a notable material. Apart from hierarchical nanostructures, diverse shaped nanostructures were also used in volatile alcohols identification. Honeycomb-like $\mathrm{SnO}_{2}$-SiNPA nanostructure, rambutan-like $\mathrm{SnO}_{2}$ hierarchical nanostructure, $\mathrm{ZnO}$ nano-tetrapods, raspberry-like $\mathrm{SnO}_{2}$ hollow nanostructure, snowflake-like $\mathrm{SnO}_{2}$ hierarchical architecture, sea cucumber-like indium tungsten oxide, hollow Pentagonal-Cone-Structured $\mathrm{SnO}_{2}$ architecture, and neck-connected nanostructure film of ZIF- 8 derived $\mathrm{ZnO}$ were proposed in the detection of alcohols as noted in Table 2 [225-232]. These materials were synthesized by solvothermal, hydrothermal, thermal-annealing, calcination, or CVD tactics. However, $\mathrm{ZnO}$ nano-tetrapods [227] can be ruled out due to their combined sensing applicability in hydrocarbon detection.

As described in Table 2, nanocomposite architectures were also demonstrated to be effective towards the quantification of alcohols. Wherein, nanocomposites of flower like $\mathrm{LaMnO}_{3} @ \mathrm{ZnO}, \mathrm{SnO}_{2}-\mathrm{Pd}-\mathrm{Pt}-\mathrm{In}_{2} \mathrm{O}_{3}, \mathrm{RGO}-\mathrm{SnO}_{2} \mathrm{NPs}, \mathrm{SnO}_{2}-\mathrm{V}_{2} \mathrm{O}_{5}, \mathrm{ZnO}: \mathrm{Fe}, \mathrm{g}-\mathrm{C}_{3} \mathrm{~N}_{4}-\mathrm{SnO}_{2}$, and $\mathrm{Co}_{3} \mathrm{O}_{4}$ nanosheet array-3D carbon foam are more impressive towards alcohols sensing studies [233-239]. Among them, $\mathrm{SnO}_{2}-\mathrm{V}_{2} \mathrm{O}_{5}$ nanocomposite [236] was demonstrated with its sensitivity to ethanol ( $\sim 66 \%$ for $160 \mathrm{ppm})$ through local grain-to-grain conductivity, but details on other sensor properties, such as response/recovery time and LOD were not available. Moreover, RGO-SnO 2 NPs composite [235] seems to be significant in terms of its capable operation between 24 and $98 \%$ humid conditions. This may be due to the presence of reduced graphene oxide along with the $\mathrm{SnO}_{2} \mathrm{NPs}$. $\mathrm{ZnO}$ :Fe nanostructured film [237] morphology was improved by UV treatment, which further enhanced its sensor response. Similarly, upon modification of $\mathrm{SnO}_{2}$ by g- $\mathrm{C}_{3} \mathrm{~N}_{4}$ NShs [238], the ethanol sensitivity was improved.

Table 2. Detection concentration, response/recovery time, operating temperature (Temp.) and LODs to volatile alcohols by diverse nanostructured materials.

\begin{tabular}{|c|c|c|c|c|c|c|}
\hline Materials/Nanostructure & Analyte/Concentration & $\begin{array}{l}\text { Gas Response } \\
\left(\mathbf{R}_{\text {air }} / \mathbf{R}_{\text {gas }}\right)\end{array}$ & Response/Recovery & Temp. & LOD & Ref \\
\hline $\mathrm{Sn}_{3} \mathrm{~N}_{4} /$ nanoparticles & Ethanol /100 ppm & 51.3 & NA & $120^{\circ} \mathrm{C}$ & $0.07 \mathrm{ppm}$ & [154] \\
\hline $\begin{array}{c}\text { C doped } \\
\mathrm{TiO}_{2} / \text { nanoparticles }\end{array}$ & n-Pentanol/100 ppm & 11.12 & $100 \mathrm{~s} / 675 \mathrm{~s}$ & $170^{\circ} \mathrm{C}$ & $0.5 \mathrm{ppm}$ & [156] \\
\hline $\begin{array}{c}\text { Pr doped } \\
\mathrm{In}_{2} \mathrm{O}_{3} / \text { nanoparticles }\end{array}$ & Ethanol/50 ppm & 106 & $16.2 \mathrm{~s} / 10 \mathrm{~s}$ & $240^{\circ} \mathrm{C}$ & $<1 \mathrm{ppm}$ & [157] \\
\hline $\begin{array}{l}\mathrm{Au} \text { and } \mathrm{ClComodified} \\
\mathrm{LaFeO} / \text { nanoparticles }\end{array}$ & Ethanol/100 ppm & $102.1 \& 220.7$ & $<40 \mathrm{~s} / \mathrm{NA}$ & $120^{\circ} \mathrm{C}$ & $<10 \mathrm{ppm}$ & [158] \\
\hline $\mathrm{LaFe}_{x} \mathrm{O}_{3-\delta} /$ nanocrystals & Ethanol/1000 ppm & 132 & $1 \mathrm{~s} / 1.5 \mathrm{~s}$ & $140^{\circ} \mathrm{C}$ & $<50 \mathrm{ppm}$ & [159] \\
\hline $\begin{array}{c}\mathrm{Cl} \text { doped } \\
\mathrm{LaFe}_{\mathrm{x}} \mathrm{O}_{3-\delta} / \text { nanocrystals }\end{array}$ & Ethanol/200 ppm & 79.2 & $9 \mathrm{~s} / 5 \mathrm{~s}$ & $136^{\circ} \mathrm{C}$ & $<100 \mathrm{ppm}$ & [160] \\
\hline$\alpha-\mathrm{MoO}_{3} /$ nanocrystals & Ethanol/100 ppm & $>55$ & $34 \mathrm{~s} / 70 \mathrm{~s}$ & $350^{\circ} \mathrm{C}$ & NA & [161] \\
\hline $\mathrm{CuO} / \mathrm{Cu}_{2} \mathrm{O} /$ nanocrystals & Ethanol/100 ppm & $10 \& 9.5$ & $5 \mathrm{~s} / 10 \mathrm{~s} \& 4.1 \mathrm{~s} / 10.5 \mathrm{~s}$ & $300^{\circ} \mathrm{C} \& 275^{\circ} \mathrm{C}$ & $<10 \mathrm{ppm}$ & [162] \\
\hline $\mathrm{Gd}_{1-\mathrm{x}} \mathrm{Ca}_{\mathrm{x}} \mathrm{FeO}_{3} /$ nanocrystals & Methanol/600 ppm & 117.7 & $1 \mathrm{~min} / 1.1 \mathrm{~min}$ & $260^{\circ} \mathrm{C}$ & $<50 \mathrm{ppm}$ & [163] \\
\hline $\begin{array}{c}\text { Au modified } \\
\mathrm{ZnO} / \text { nanowires }\end{array}$ & Ethanol/500 ppm & 12.35 & $215 \mathrm{~s} / 180 \mathrm{~s}$ & $350^{\circ} \mathrm{C}$ & $<10$ ppm & [164] \\
\hline $\begin{array}{c}\mathrm{Fe}_{2} \mathrm{O}_{3} \text { nanoparticles } \\
\text { coated } \mathrm{SnO}_{2} / \text { nanowires }\end{array}$ & Ethanol/200 ppm & 57.56 & $300 \mathrm{~s} / 100 \mathrm{~s}$ & $300^{\circ} \mathrm{C}$ & $<5 \mathrm{ppm}$ & [165] \\
\hline
\end{tabular}


Table 2. Cont.

\begin{tabular}{|c|c|c|c|c|c|c|}
\hline Materials/Nanostructure & Analyte/Concentration & $\begin{array}{c}\text { Gas Response } \\
\left(\mathbf{R}_{\text {air }} / \mathbf{R}_{\text {gas }}\right)\end{array}$ & Response/Recovery & Temp. & LOD & Ref \\
\hline $\begin{array}{c}\mathrm{In}_{2} \mathrm{O}_{3} \text { nanoparticles } \\
\text { decorated } \mathrm{ZnS} / \text { nanowires }\end{array}$ & Ethanol/500 ppm & $>25$ & $400 \mathrm{~s} / 100 \mathrm{~s}$ & $300^{\circ} \mathrm{C}$ & $<10$ ppm & [166] \\
\hline $\begin{array}{c}\text { Sr-doped cubic } \\
\mathrm{In}_{2} \mathrm{O}_{3} / \text { rhombohedral } \\
\mathrm{In}_{2} \mathrm{O}_{3} / \text { nanowires }\end{array}$ & Ethanol/1 ppm & 21 & $<1 \mathrm{~m}$ (both) & $300^{\circ} \mathrm{C}$ & 0.025 ppm & {$[167]$} \\
\hline $\begin{array}{c}\mathrm{Cr}_{2} \mathrm{O}_{3} \text { nanoparticles } \\
\text { functionalized } \\
\mathrm{WO}_{3} / \text { nanorods }\end{array}$ & Ethanol/200 ppm & 5.58 & $51.35 \mathrm{~s} / 48.65 \mathrm{~s}$ & $300^{\circ} \mathrm{C}$ & $<5 \mathrm{ppm}$ & {$[168]$} \\
\hline $\mathrm{ZnO} /$ nanorods & Ethanol/100 ppm & 23 & $26 \mathrm{~s} / 43 \mathrm{~s}$ & Room Temp. & $<5 \mathrm{ppm}$ & [169] \\
\hline 1D-ZnO/nanorods & Ethanol/100 ppm & 44.9 & $6 \mathrm{~s} / 31 \mathrm{~s}$ & $300^{\circ} \mathrm{C}$ & $<10 \mathrm{ppm}$ & {$[170]$} \\
\hline $\begin{array}{c}\text { Pd nanoparticles } \\
\text { decorated } \mathrm{ZnO} / \text { nanorods }\end{array}$ & Ethanol/500 ppm & 81 & $6 \mathrm{~s} / 95 \mathrm{~s}$ & $260^{\circ} \mathrm{C}$ & $<100$ ppm & {$[171]$} \\
\hline $\mathrm{SnO}_{2} / \mathrm{ZnO} /$ nanorods & Ethanol/100 ppm & 18.1 & $2 \mathrm{~s} / 38 \mathrm{~s}$ & $275^{\circ} \mathrm{C}$ & $<1 \mathrm{ppm}$ & [172] \\
\hline $\begin{array}{c}\text { rGO- } \\
\mathrm{WO}_{3} \cdot 0.33 \mathrm{H}_{2} \mathrm{O} / \text { nanoneedles }\end{array}$ & Isopropanol/100 ppm & 4.96 & $<90 \mathrm{~s} / \mathrm{NA}$ & Room Temp. & 1 ppm & {$[173]$} \\
\hline $\begin{array}{c}\text { Sm-doped } \\
\mathrm{SnO}_{2} / \text { nanoarrays }\end{array}$ & Isopropanol/100 ppm & 117.7 & $12 \mathrm{~s} / 20 \mathrm{~s}$ & $252{ }^{\circ} \mathrm{C}$ & 1 ppm & {$[174]$} \\
\hline SmFeO3/nanofibers & $\begin{array}{l}\text { Ethylene glycol/100 } \\
\text { ppm }\end{array}$ & 18.19 & $41 \mathrm{~s} / 47 \mathrm{~s}$ & $240^{\circ} \mathrm{C}$ & $\sim 5 \mathrm{ppm}$ & [175] \\
\hline In doped $\mathrm{NiO} /$ nanofibers & methanol/200 ppm & 10.9 & $273 \mathrm{~s} / 26 \mathrm{~s}$ & $300^{\circ} \mathrm{C}$ & $25 \mathrm{ppm}$ & [176] \\
\hline $\begin{array}{c}\mathrm{SiO}_{2} @ \mathrm{SnO}_{2 / \text { core-shell }} \\
\text { nanofibers }\end{array}$ & Ethanol/200 ppm & 37 & $13 \mathrm{~s} / 16 \mathrm{~s}$ & NA & NA & {$[177]$} \\
\hline $\begin{array}{c}\mathrm{Yb} \text { doped } \\
\mathrm{In}_{2} \mathrm{O}_{3} / \text { nanofibers }\end{array}$ & Ethanol/10 ppm & $40 \& 5$ & NA & Room Temp. & $<1 \& 5$ ppm & [178] \\
\hline $\mathrm{CuO} / \mathrm{CuCo}_{2} \mathrm{O}_{4} /$ nanotubes & n-Propanol/10 ppm & 14 & $6.3 \mathrm{~s} / 4.1 \mathrm{~s}$ & Room Temp. & $<10$ ppm & {$[179]$} \\
\hline $\mathrm{CuO}-\mathrm{NiO} /$ nanotubes & Glycol/100 ppm & 10.35 & $15 \mathrm{~s} / 45 \mathrm{~s}$ & $110^{\circ} \mathrm{C}$ & $0.078 \mathrm{ppm}$ & [180] \\
\hline $\begin{array}{c}\mathrm{NiO} \text { decorated } \\
\mathrm{SnO}_{2} / \text { vertical standing } \\
\text { nanotubes }\end{array}$ & Ethanol/1000 ppm & 123.7 & $10 \mathrm{~s} / 58 \mathrm{~s}$ & $250^{\circ} \mathrm{C}$ & NA & {$[181]$} \\
\hline $\begin{array}{c}\text { Ca doped } \\
\operatorname{In}_{2} \mathrm{O}_{3} / \text { nanotubes }\end{array}$ & Ethanol/100 ppm & 183.3 & $2 \mathrm{~s} / 56 \mathrm{~s}$ & $240^{\circ} \mathrm{C}$ & $<5 \mathrm{ppm}$ & {$[182]$} \\
\hline $\begin{array}{l}\text { Au and Ni doped } \\
\mathrm{In}_{2} \mathrm{O}_{3} / \text { nanotubes }\end{array}$ & Ethanol/100 ppm & $16.16 \& 49.74$ & $5 s / 64 s \& 3 s / 49 s$ & $160^{\circ} \mathrm{C} \& 220^{\circ} \mathrm{C}$ & $\begin{array}{l}<5 \text { ppm (for } \\
\text { both) }\end{array}$ & [183] \\
\hline W doped $\mathrm{NiO} /$ nanotubes & Ethanol/100 ppm & 40.56 & $54 \mathrm{~s} / 22 \mathrm{~s}$ & $200{ }^{\circ} \mathrm{C}$ & $<5 \mathrm{ppm}$ & [184] \\
\hline $\begin{array}{c}\mathrm{In}_{2} \mathrm{O}_{3} \text { NPs deposited } \\
\mathrm{TiO}_{2} / \text { nanobelts }\end{array}$ & Ethanol/100 ppm & $>9$ & $6 \mathrm{~s} / 3 \mathrm{~s}$ & $100^{\circ} \mathrm{C}$ & 1 ppm & [185] \\
\hline$\alpha-\mathrm{MoO}_{3} /$ nanobelts & Ethanol/800 ppm & 173 & $<65 \mathrm{~s} />15 \mathrm{~s}$ & $300^{\circ} \mathrm{C}$ & $<50 \mathrm{ppm}$ & {$[186]$} \\
\hline $\begin{array}{c}\mathrm{Zn} \text { doped } \\
\mathrm{MoO}_{3 / \text { nanobelts }}\end{array}$ & Ethanol/1000 ppm & 321 & $<121 \mathrm{~s}$ (for both) & $240^{\circ} \mathrm{C}$ & 5 ppm & [187] \\
\hline $\begin{array}{c}\text { MOF derived } \\
\mathrm{Fe}_{2} \mathrm{O}_{3} / \text { nanocubes }\end{array}$ & Ethanol/100 ppm & $\sim 6$ & $<120 \mathrm{~s} /<60 \mathrm{~s}$ & $160-230^{\circ} \mathrm{C}$ & $<1 \mathrm{ppm}$ & [188] \\
\hline $\mathrm{In}_{2} \mathrm{O}_{3} /$ nanocubes & Ethanol/100 ppm & 85 & $15 \mathrm{~s} / 60 \mathrm{~s}$ & $300^{\circ} \mathrm{C}$ & $<5 \mathrm{ppm}$ & [189] \\
\hline $\begin{array}{c}\text { ZIF-8 derived } \\
\text { ZnO/hollow nanocages }\end{array}$ & Ethanol/100 ppm & 139.41 & $2.8 \mathrm{~s} / 56.4 \mathrm{~s}$ & $325^{\circ} \mathrm{C}$ & $0.025 \mathrm{ppm}$ & [191] \\
\hline $\begin{array}{c}\text { ZIF-8 derived } \mathrm{Ag} \\
\text { functionalized } \\
\mathrm{ZnO} / \text { hollow nanocages }\end{array}$ & Ethanol/100 ppm & 84.6 & $5 \mathrm{~s} / 10 \mathrm{~s}$ & $275^{\circ} \mathrm{C}$ & $0.0231 \mathrm{ppm}$ & [192] \\
\hline $\begin{array}{c}\mathrm{Cu}_{2} \mathrm{O} / \text { hollow } \\
\text { dodecahedral nanocages }\end{array}$ & Ethanol/100 ppm & 4.6 & $112.4 \mathrm{~s} / 157.5 \mathrm{~s}$ & $250^{\circ} \mathrm{C}$ & NA & [193] \\
\hline $\begin{array}{c}\text { Al-doped } \\
\mathrm{ZnO} / \text { nanosheets }\end{array}$ & Ethanol/100 ppm & 90.2 & $1.6 \mathrm{~s} / 1.8 \mathrm{~s}$ & $370^{\circ} \mathrm{C}$ & $<1 \mathrm{ppm}$ & [194] \\
\hline $\begin{array}{l}\mathrm{NiO} N P s \text { decorated } \\
\mathrm{SnO}_{2} / \text { nanosheets }\end{array}$ & Ethanol/100 ppm & 153 & NA & $260^{\circ} \mathrm{C}$ & $<5 \mathrm{ppm}$ & [195] \\
\hline
\end{tabular}


Table 2. Cont.

\begin{tabular}{|c|c|c|c|c|c|c|}
\hline Materials/Nanostructure & Analyte/Concentration & $\begin{array}{l}\text { Gas Response } \\
\left(\mathbf{R}_{\text {air }} / \mathbf{R}_{\text {gas }}\right)\end{array}$ & Response/Recovery & Temp. & LOD & Ref \\
\hline $\begin{array}{l}\mathrm{CuO} \text { NPs decorated } \\
\mathrm{ZnO} / \text { nanosheets }\end{array}$ & Ethanol/200 ppm & 97 & $<7 \mathrm{~s} /<40 \mathrm{~s}$ & $320^{\circ} \mathrm{C}$ & $<1 \mathrm{ppm}$ & [196] \\
\hline $\mathrm{SnS}_{2} /$ nanoflakes & Methanol/150 ppm & 1580 & $67 \mathrm{~s} / 5 \mathrm{~s}$ & Room Temp. & NA & [197] \\
\hline $\begin{array}{c}\text { Co doped } \mathrm{ZnO} \text { /hexagonal } \\
\text { nanoplates }\end{array}$ & Ethanol/300 ppm & 570 & $50 \mathrm{~s} / 5 \mathrm{~s}$ & $300^{\circ} \mathrm{C}$ & $\sim 50 \mathrm{ppm}$ & [199] \\
\hline $\begin{array}{c}\text { ZIF-8 derived } \alpha \text { - } \\
\mathrm{Fe}_{2} \mathrm{O}_{3} / \mathrm{ZnO} / \mathrm{Au} / \text { nanoplates }\end{array}$ & Ethanol/100 ppm & 170 & $4 \mathrm{~s} / 5 \mathrm{~s}$ & $280^{\circ} \mathrm{C}$ & $\sim 10 \mathrm{ppm}$ & [200] \\
\hline $\mathrm{ZnO} /$ nanoplates & Ethanol/1000 ppm & 8.5 & $\begin{array}{c}154.4 \mathrm{~s}(125 \\
\mathrm{ppm}) / 114.2 \mathrm{~s}(1500 \\
\mathrm{ppm})\end{array}$ & $164^{\circ} \mathrm{C}$ & NA & [201] \\
\hline $\mathrm{Zn}_{2} \mathrm{SnO}_{4} /$ nanospheres & Ethanol/50 ppm & 23.4 & $18 \mathrm{~s} / 45 \mathrm{~s}$ & $180^{\circ} \mathrm{C}$ & $\sim 5$ ppm & [202] \\
\hline $\begin{array}{c}\text { Indium Tungsten } \\
\text { Oxide/ellipsoidal } \\
\text { nanospheres }\end{array}$ & Methanol/400 ppm & $>5$ & $2 \mathrm{~s} / 9 \mathrm{~s}$ & $312^{\circ} \mathrm{C}$ & $\sim 20 \mathrm{ppm}$ & [203] \\
\hline $\begin{array}{l}\mathrm{Ag} @ \mathrm{In}_{2} \mathrm{O}_{3} / \text { core-shell } \\
\text { nanospheres }\end{array}$ & Ethanol/50 ppm & 72.56 & $13 \mathrm{~s} / 8 \mathrm{~s}$ & $220^{\circ} \mathrm{C}$ & $\sim 2 \mathrm{ppm}$ & [204] \\
\hline $\begin{array}{l}\mathrm{ZnSnO} / \text { hollow } \\
\text { nanospheres }\end{array}$ & n-Propanol/500 ppm & 64 & NA & $200^{\circ} \mathrm{C}$ & $0.5 \mathrm{ppm}$ & [205] \\
\hline ZnO/hollow nanospheres & n-Butanol/500 ppm & 292 & $36 \mathrm{~s} / 9 \mathrm{~s}$ & $385^{\circ} \mathrm{C}$ & $\sim 10 \mathrm{ppm}$ & [206] \\
\hline $\begin{array}{c}\alpha-\mathrm{Fe}_{2} \mathrm{O}_{3} / \text { hollow } \\
\text { nanospheres }\end{array}$ & Methanol/10 ppm & 25 & $8 \mathrm{~s} / 9 \mathrm{~s}$ & $280^{\circ} \mathrm{C}$ & $1 \mathrm{ppm}$ & [207] \\
\hline $\begin{array}{l}\text { PdO NPs modified } \\
\mathrm{ZnO} / \text { nanoflowers }\end{array}$ & Methanol/150 ppm & $>80$ & $18 \mathrm{~s} / 52.2 \mathrm{~s}$ & $150^{\circ} \mathrm{C}$ & $<0.5 \mathrm{ppm}$ & [208] \\
\hline $\mathrm{NiO} /$ grained nanoflowers & Ethanol/150 ppm & 35 & $3 \mathrm{~s} / 6 \mathrm{~s}$ & $200^{\circ} \mathrm{C}$ & $2.6 \mathrm{ppm}$ & [209] \\
\hline $\begin{array}{l}\text { rGO nanosheets modified } \\
\mathrm{NiCo}_{2} \mathrm{~S}_{4} / \text { nanoflowers }\end{array}$ & Ethanol/100 ppm & $>2.5$ & $4.56 \mathrm{~s} / 10.38 \mathrm{~s}$ & $100^{\circ} \mathrm{C}$ & $<10 \mathrm{ppm}$ & [210] \\
\hline $\begin{array}{l}\mathrm{Pd} \text { and rGO modified } \\
\mathrm{TiO}_{2} / \text { nanoflowers }\end{array}$ & Ethanol/700 ppm & $>64 \%$ (for both) & $\begin{array}{l}6.55 \mathrm{~s} / 186.97 \mathrm{~s} \& \\
75.64 \mathrm{~s} / 147.16 \mathrm{~s}\end{array}$ & $90^{\circ} \mathrm{C}$ & $<10 \mathrm{ppm}$ & [211] \\
\hline $\begin{array}{c}\text { Ag- } \\
\text { functionalizedZnO/macro- } \\
\text { /mesoporous- } \\
\text { nanostructure }\end{array}$ & n-Butanol/100 ppm & 994.8 & $66 \mathrm{~s} / 25 \mathrm{~s}$ & $240^{\circ} \mathrm{C}$ & $<1 \mathrm{ppm}$ & [212] \\
\hline $\begin{array}{l}\text { Al-doped } \mathrm{ZnO} / \text { macro- } \\
\text { /mesoporous- } \\
\text { nanostructure }\end{array}$ & n-Butanol/100 ppm & 751.96 & $25 \mathrm{~s} / 23 \mathrm{~s}$ & $300^{\circ} \mathrm{C}$ & $\sim 1 \mathrm{ppm}$ & [213] \\
\hline $\begin{array}{c}\text { Au loaded } \\
\mathrm{WO}_{3} / \text { mesoporous- } \\
\text { nanostructure }\end{array}$ & n-Butanol/100 ppm & 14.35 & $10 \mathrm{~s} / 35 \mathrm{~s}$ & $250^{\circ} \mathrm{C}$ & $<10 \mathrm{ppm}$ & [214] \\
\hline $\begin{array}{l}\text { In doped } \mathrm{ZnO} / \text { three } \\
\text { dimensionally ordered } \\
\text { mesoporous- } \\
\text { nanostructure }\end{array}$ & Ethanol/100 ppm & 88 & $25 \mathrm{~s} / 10 \mathrm{~s}$ & $250^{\circ} \mathrm{C}$ & $<5 \mathrm{ppm}$ & [215] \\
\hline $\begin{array}{l}\mathrm{Si@ZnO} \mathrm{NPs/} \\
\text { mesoporous- } \\
\text { nanostructure }\end{array}$ & Ethanol/300 ppm & 62.5 & $0.27 \mathrm{~min} / 3.5 \mathrm{~min}$ & $400^{\circ} \mathrm{C}$ & $<50 \mathrm{ppm}$ & [216] \\
\hline $\begin{array}{c}\text { Ag loaded } \\
\text { g- } \mathrm{C}_{3} \mathrm{~N}_{4} \text { / mesoporous- } \\
\text { nanostructure }\end{array}$ & Ethanol/50 ppm & 49.2 & $11.5 \mathrm{~s} / 7 \mathrm{~s}$ & $250^{\circ} \mathrm{C}$ & $<1 \mathrm{ppm}$ & [217] \\
\hline $\begin{array}{l}\mathrm{Pd} / \mathrm{SnO}_{2} \text { porous- } \\
\text { nanostructure }\end{array}$ & Ethanol/5-200 ppm & $\sim 90 \%$ & $1.5 \mathrm{~s} / 18 \mathrm{~s}$ & $300^{\circ} \mathrm{C}$ & $<5 \mathrm{ppm}$ & [218] \\
\hline $\begin{array}{l}\mathrm{SnO}_{2} / \text { mesoporous- } \\
\text { nanofibers }\end{array}$ & $\begin{array}{l}\text { n-Butanol/300 ppm } \\
\text { (for both) }\end{array}$ & $556.5 \& 415.3$ & $\begin{array}{c}195 \mathrm{~s} / 100 \mathrm{~s} \& 64 \mathrm{~s} / 36 \\
\mathrm{~s}\end{array}$ & $150^{\circ} \mathrm{C} \& 200^{\circ} \mathrm{C}$ & $<10 \mathrm{ppm}$ & [219] \\
\hline $\begin{array}{c}\mathrm{TiO}_{2}-\mathrm{SnO}_{2} / \text { hierarchical } \\
\text { branched mesoporous } \\
\text { nano- composite }\end{array}$ & Ethanol/50 ppm & 40 & $7 \mathrm{~s} / 5 \mathrm{~s}$ & $350^{\circ} \mathrm{C}$ & $0.2 \mathrm{ppm}$ & [220] \\
\hline
\end{tabular}


Table 2. Cont.

\begin{tabular}{|c|c|c|c|c|c|c|}
\hline Materials/Nanostructure & Analyte/Concentration & $\begin{array}{l}\text { Gas Response } \\
\quad\left(\mathbf{R}_{\text {air }} / \mathbf{R}_{\text {gas }}\right)\end{array}$ & Response/Recovery & Temp. & LOD & Ref \\
\hline $\begin{array}{c}\text { Co-Doped } \\
\text { ZnO/hierarchical } \\
\text { mesoporous- } \\
\text { nanostructure }\end{array}$ & Ethanol/50 ppm & 54 & $22 \mathrm{~s} / 53 \mathrm{~s}$ & $180^{\circ} \mathrm{C}$ & $0.0454 \mathrm{ppm}$ & [221] \\
\hline $\begin{array}{c}\mathrm{Fe}_{2} \mathrm{O}_{3} \text { nanorods on } \mathrm{SnO}_{2} \\
\text { nanospheres/hierarchical } \\
\text { nano- composite }\end{array}$ & Ethanol/100 ppm & 23.512 & $5 \mathrm{~s} / 12 \mathrm{~s}$ & $320^{\circ} \mathrm{C}$ & $<50$ ppm & [222] \\
\hline $\begin{array}{c}\mathrm{MoO}_{3} \text {-mixed } \\
\mathrm{SnO}_{2} / \text { hierarchical } \\
\text { nanostructure }\end{array}$ & Ethanol/100 ppm & 714 & $\begin{array}{c}1 \mathrm{~s}(\text { for all }) / 357 \mathrm{~s}, 8 \mathrm{~s} \\
\text { and } 85 \mathrm{~s}\end{array}$ & $260^{\circ} \mathrm{C}$ & $<10$ ppm & [223] \\
\hline $\begin{array}{c}\mathrm{In}_{2} \mathrm{O}_{3} \text { Nanoparticles } \\
\text { Decorated } \\
\mathrm{ZnO} / \text { hierarchical } \\
\text { nanostructure }\end{array}$ & n-Butanol/100 ppm & 218.3 & $5 \mathrm{~s} / 12 \mathrm{~s}$ & $260^{\circ} \mathrm{C}$ & $\begin{array}{l}\text { Down to } \\
\text { sub-ppm }\end{array}$ & [224] \\
\hline $\begin{array}{c}\mathrm{SnO}_{2}-\mathrm{Si}- \\
\text { NPA/honeycomb like } \\
\text { nanostructure }\end{array}$ & Ethanol/50 ppm & 7.7 & $10 \mathrm{~s} / 9 \mathrm{~s}$ & $320^{\circ} \mathrm{C}$ & $<10 \mathrm{ppm}$ & [225] \\
\hline $\begin{array}{c}\mathrm{SnO}_{2} / \text { rambutan-like } \\
\text { hierarchical nanostructure }\end{array}$ & n-Butanol/100 ppm & 44.3 & $8 \mathrm{~s} / 5 \mathrm{~s}$ & $140^{\circ} \mathrm{C}$ & $<20$ ppm & [226] \\
\hline $\begin{array}{l}\mathrm{SnO}_{2} / \text { raspberry-like } \\
\text { hollow nanostructure }\end{array}$ & n-Butanol/100 ppm & 303.49 & $163 \mathrm{~s} / 808 \mathrm{~s}$ & $160^{\circ} \mathrm{C}$ & 1 ppm & {$[228]$} \\
\hline $\begin{array}{c}\mathrm{SnO}_{2} / \text { snowflake-like } \\
\text { hierarchical nanostructure }\end{array}$ & Ethanol/250 ppm & $\sim 55$ & $6 \mathrm{~s} / 7 \mathrm{~s}$ & $400^{\circ} \mathrm{C}$ & NA & [229] \\
\hline $\begin{array}{l}\mathrm{SnO}_{2} / \text { pentagonal-cone } \\
\text { assembled with nanorods }\end{array}$ & Ethanol/200 ppm & 98 & $11 \mathrm{~s} / 18 \mathrm{~s}$ & $220^{\circ} \mathrm{C}$ & $1 \mathrm{ppm}$ & [231] \\
\hline $\begin{array}{l}\text { ZIF-8 derived } \mathrm{ZnO} / \text { neck- } \\
\text { connected nanostructure } \\
\text { films }\end{array}$ & Ethanol/50 ppm & 124 & $120 \mathrm{~s} / 70 \mathrm{~s}$ & $375^{\circ} \mathrm{C}$ & $0.5 \mathrm{ppm}$ & [232] \\
\hline $\begin{array}{c}\mathrm{LaMnO}_{3} @ \mathrm{ZnO} / \text { nano- } \\
\text { composite }\end{array}$ & n-Butanol /100 ppm & 6 & $8 \mathrm{~s} / 17 \mathrm{~s}$ & $300{ }^{\circ} \mathrm{C}$ & NA & [233] \\
\hline $\begin{array}{l}\mathrm{SnO}_{2}-\mathrm{Pd}-\mathrm{Pt}-\mathrm{In}_{2} \mathrm{O}_{3 /} \text { nano- } \\
\text { composite }\end{array}$ & Methanol/100 ppm & 320.73 & $32 \mathrm{~s} / 47 \mathrm{~s}$ & $160^{\circ} \mathrm{C}$ & $0.1 \mathrm{ppm}$ & {$[234]$} \\
\hline $\begin{array}{l}\text { RGO-SnO } / \text { /nano- } \\
\text { composite }\end{array}$ & Ethanol /100 ppm & 43 & $8 \mathrm{~s} / \mathrm{NA}$ & $300^{\circ} \mathrm{C}$ & $\sim 5 \mathrm{ppm}$ & [235] \\
\hline $\begin{array}{l}\mathrm{ZnO}: \mathrm{Fe} / \text { nano-composite } \\
\text { films }\end{array}$ & Ethanol/100 ppm & $61 \& 36$ & $\begin{array}{c}1.1 \mathrm{~s} / 1.45 \mathrm{~s} \& 0.23 \\
\mathrm{~s} / 0.34 \mathrm{~s}\end{array}$ & $250{ }^{\circ} \mathrm{C} \& 350{ }^{\circ} \mathrm{C}$ & $\sim 10$ ppm & [237] \\
\hline $\begin{array}{l}\text { g- } \mathrm{C}_{3} \mathrm{~N}_{4}-\mathrm{SnO}_{2} / \text { nano- } \\
\text { composite }\end{array}$ & Ethanol/500 ppm & 240 & $15 \mathrm{~s} / 38 \mathrm{~s}$ & $300^{\circ} \mathrm{C}$ & $\sim 50$ ppm & [238] \\
\hline $\begin{array}{c}\mathrm{Co}_{3} \mathrm{O}_{4} \text { nanosheet } \\
\text { array-3D carbon foam/ } \\
\text { nano- composite }\end{array}$ & Ethanol/100 ppm & 10.4 & $45 \mathrm{~s} / 140 \mathrm{~s}$ & $100^{\circ} \mathrm{C}$ & $0.2 \mathrm{ppm}$ & [239] \\
\hline
\end{tabular}

NA = Not available; Temp. = Temperature; $\mathrm{s}=$ seconds; $\mathrm{min}=$ minutes .

\section{Various Nanostructures in Volatile Aldehyde Detection}

In addition to acetone or alcoholic vapors detection, volatile organic aldehydes quantitation is also become essential and diverse nanostructured materials were reported in the aldehyde gases quantification. This section describes published reports in detail. Majority of the research papers generally focused on the assay of formaldehyde $(\mathrm{HCHO})$ via the following reaction mechanism [240], which are also applicable for other aldehydes detection. As described in Equations (8) and (9), the adsorbed oxygen in the sensor chamber first reacts to form the acid followed by interaction with oxygen anion to release electrons, which can be adopted as the sensor signal.

$$
\mathrm{HCHO}+\mathrm{O}^{-} \text {(ads) } \rightarrow \mathrm{HCOOH}+\mathrm{e}^{-}
$$




$$
\mathrm{HCHO}+2 \mathrm{O}^{-} \rightarrow \mathrm{CO}_{2}+\mathrm{H}_{2} \mathrm{O}+2 \mathrm{e}^{-}
$$

Nanoparticles, such as $\mathrm{In}_{2} \mathrm{O}_{3}$ NPs, molecularly imprinted polymers (MIPs) NPs, amorphous $\mathrm{Eu}_{0.9} \mathrm{Ni}_{0.1} \mathrm{~B}_{6} \mathrm{NPs}$, Ni-doped $\mathrm{SnO}_{2} \mathrm{NPs}$, and $\mathrm{NiO}$ granular NPs films were utilized to detect the formaldehyde at diverse operating temperatures with sub-ppm LODs, as noted in Table 3 [241-245]. Among them, molecularly imprinted polymers (MIPs) NPs discriminated the formaldehyde by means of quartz crystal microbalance (QCM) tactic with a LOD of $0.5 \mathrm{ppm}$, thus can be noted as an additional approach for the formaldehyde detection. Moreover, amorphous $\mathrm{Eu}_{0.9} \mathrm{Ni}_{0.1} \mathrm{~B}_{6} \mathrm{NPs}$ [243] were noted as an exceptional candidate in formaldehyde quantitation due do their sensor response $\left(\mathrm{R}_{\mathrm{a}} / \mathrm{R}_{\mathrm{g}} \geq 7\right.$ for $20 \mathrm{ppm}$; response/recovery time $\leq 20 \mathrm{~s}$ (for both)) at room temperature. Therefore, commercialization of amorphous $\mathrm{Eu}_{0.9} \mathrm{Ni}_{0.1} \mathrm{~B}_{6}$ NPs towards $\mathrm{HCHO}$ detection is desirable.

Regarding the $\mathrm{HCHO}$ quantification, researchers developed nanowires $\left(\mathrm{SnO}_{2} \mathrm{NWs}\right.$, $\mathrm{p}-\mathrm{CuO} / \mathrm{n}-\mathrm{SnO}_{2}$ core-shell $\mathrm{NWs}, \mathrm{ZnO}$ meso-structured NWs and RGO coated $\mathrm{Si} \mathrm{NWs}$ ) and nanorods (Co doped $\operatorname{In}_{2} \mathrm{O}_{3}$ NRds and Ag-functionalized and Ni-doped $\operatorname{In}_{2} \mathrm{O}_{3} \mathrm{NRds}$ ) via VLS, atomic layer deposition, low-temperature chemical synthesis, metal-assisted chemical etching method (MACE), thermal annealing methods and applied them in effective sensing of HCHO gas [246-251]. As noted in Table 3, many of these materials display exceptional sensitivity to $\mathrm{HCHO}$ at different working temperatures. Note that $\mathrm{ZnO}$ meso-structured NWs [248] are capable of operating at room temperature and show a high sensor response of $1223 \%$ to $50 \mathrm{ppm} \mathrm{HCHO}$ with a LOD of $0.005 \mathrm{ppm}$ under UV, thereby attest as a motivational research. In view of this, Zhang et al. reported the utility of $\mathrm{Ag}-\mathrm{LaFeO}_{3}$ with spheres, fibers, and cages architectures with sensor responses of 16, 14, and 23 for 1 ppm $\mathrm{HCHO}$ at $82{ }^{\circ} \mathrm{C}, 110^{\circ} \mathrm{C}$, and $70{ }^{\circ} \mathrm{C}$, correspondingly [252]. This work has driven the utilization of diverse nanostructures in VOCs determination as described below.

Materials with nanofibers structures were also attested their sensing ability to $\mathrm{HCHO}$ vapor. Ag-doped $\mathrm{LaFeO}_{3}$ nanofibers, $\mathrm{Co}_{3} \mathrm{O}_{4}$ - $\mathrm{ZnO}$ core-shell nanofibers, $\mathrm{WO}_{3} / \mathrm{ZnWO}_{4}-1 \mathrm{D}$ nanofibers, and $\mathrm{Pr}$-doped $\mathrm{BiFeO}_{3} /$ hollow nanofibers were synthesized by electrospinning method with the combination of calcination tactic and utilized in $\mathrm{HCHO}$ detection at $190-230{ }^{\circ} \mathrm{C}$ [253-256]. As shown in Table 3, $\mathrm{WO}_{3} / \mathrm{ZnWO}_{4}-1 \mathrm{D}$ hetrostructured nanofibers are found to be a good candidate in terms of the sensor response $\left(\mathrm{R}_{\mathrm{a}} / \mathrm{R}_{\mathrm{g}}=44.5\right.$ for $5 \mathrm{ppm}$ at $220^{\circ} \mathrm{C}$; response /recovery time $=12 \mathrm{~s} / 14 \mathrm{~s}$ ) with a LOD of $1 \mathrm{ppm}$ [255]. However, further optimization to reduce the working temperature is still needed. Liang and co-workers presented the alkaline earth metals-doped $\operatorname{In}_{2} \mathrm{O}_{3}$ NTs for the sensing of $\mathrm{HCHO}$ [257]. In which, Ca-doped $\operatorname{In}_{2} \mathrm{O}_{3}$ NTs showed a sensor response of 116 for $100 \mathrm{ppm} \mathrm{HCHO}$ at $130^{\circ} \mathrm{C}$ with the response/recovery time of $1 \mathrm{~s} / 328 \mathrm{~s}$ and a LOD of $0.06 \mathrm{ppm}$. Therefore, it can be authorized as an exceptional material for the $\mathrm{HCHO}$ sensor studies. Doped nanobelts were employed in the discriminative assay of $\mathrm{HCHO}$ as detailed below. Er-doped $\mathrm{SnO}_{2}$ and Ptdecorated $\mathrm{MoO}_{3}$ nanobelts were reported for the quantitation of volatile $\mathrm{HCHO}[258,259]$. As shown in Figure 14, Er-doped $\mathrm{SnO}_{2}$ nanobelts [258] display a distinct response to $\mathrm{HCHO}$ vapor $\left(R_{a} / R_{g}=9\right.$ for $100 \mathrm{ppm}$ at $230{ }^{\circ} \mathrm{C}$; response/recovery time $\left.=17 \mathrm{~s} / 25 \mathrm{~s}\right)$ with a LOD of $0.141 \mathrm{ppm}$. On the other hand, Pt-decorated $\mathrm{MoO}_{3}$ nanobelts [259] are more impressive with a sensor response $\left(R_{a} / R_{g}=\sim 25 \%\right.$ for $100 \mathrm{ppm}$; response $/$ recovery time $=17.8 \mathrm{~s} / 10.5 \mathrm{~s}$; $\mathrm{LOD}=1 \mathrm{ppm}$ ) at room temperature, thereby become a noteworthy material in $\mathrm{HCHO}$ sensory research.

Nanocube-shaped materials were consumed in the quantification of $\mathrm{HCHO}$ vapor at diverse operating temperatures. Multi-shelled hollow nanocubes $\left(\mathrm{ZnSnO}_{3}\right.$ and $\mathrm{ZnSn}(\mathrm{OH})_{6}$; synthesized by co-precipitation method) were engaged in $\mathrm{HCHO}$ sensing at $220^{\circ} \mathrm{C}$ and $60{ }^{\circ} \mathrm{C}$, respectively $[260,261]$. The responses reached 37.2 and 56.6 (for $100 \mathrm{ppm}$; response/recovery time $=1 \mathrm{~s} / 59 \mathrm{~s}$ and $1 \mathrm{~s} / 89 \mathrm{~s}$, respectively) with the LODs of $<10 \mathrm{ppm}$ and $1 \mathrm{ppm}$, correspondingly. Both materials performed remarkablely in $\mathrm{HCHO}$ sensory studies. Similarly, MOF (zeolite imidazolate framework-67; ZIF-67)-derived $\mathrm{Co}_{3} \mathrm{O}_{4} / \mathrm{CoFeO}_{4}$ double-shelled nanocubes were utilized in the detection of $\mathrm{HCHO}$ [262]. As illustrated in Figure 15, the ZIF-67-derived $\mathrm{Co}_{3} \mathrm{O}_{4} / \mathrm{CoFeO}_{4}$ double-shelled nanocubes were synthesized 
by MOF route and found to be more effective in the sensing of $\mathrm{HCHO}$ even at $1 \mathrm{ppm}$ concentration.

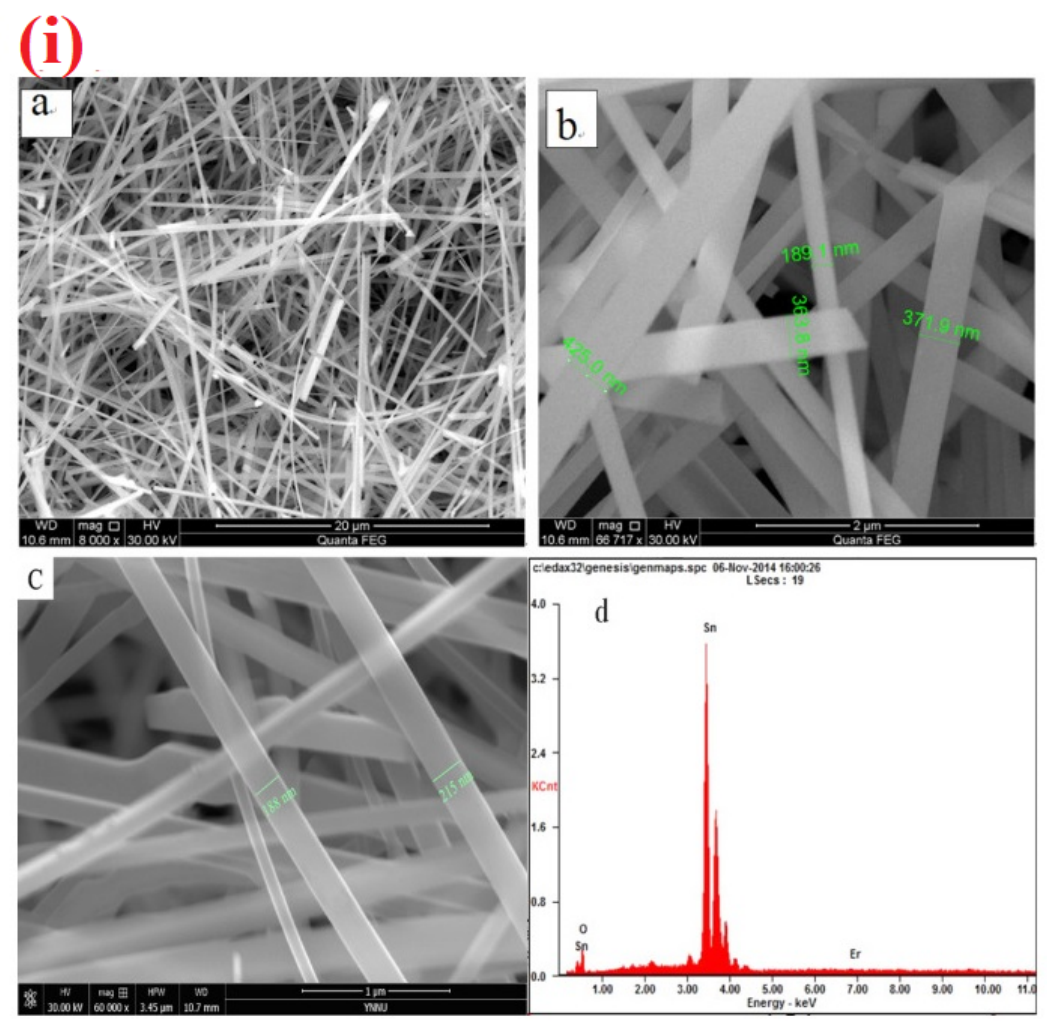

(ii)
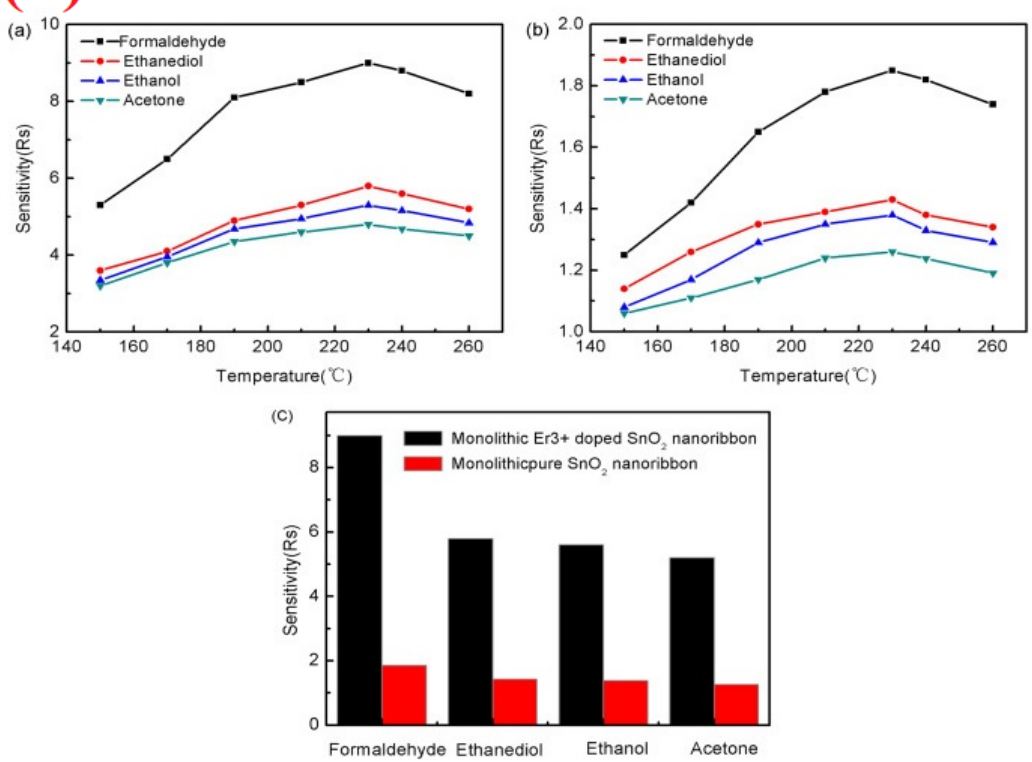

Figure 14. (i) SEM images of Er-SnO $\mathrm{S}_{2}$ nanobelts (a) at a low magnification, (b) at a high magnification, (c) SEM image of pure $\mathrm{SnO}_{2}$ nanobelts, and (d) EDS spectra of $\mathrm{Er}^{-\mathrm{SnO}_{2}}$ nanobelts; (ii) (a) The responses $\left(R_{s}\right)$ versus temperature $(T)$ of $\mathrm{Er}-\mathrm{SnO}_{2}$ nanobelt to $100 \mathrm{ppm}$ gas from $150{ }^{\circ} \mathrm{C}$ to $260^{\circ} \mathrm{C}$, (b) The responses $\left(R_{s}\right)$ versus temperature ( $T$ ) of $\mathrm{SnO}_{2}$ nanobelt to $100 \mathrm{ppm}$ gas from $150{ }^{\circ} \mathrm{C}$ to $260^{\circ} \mathrm{C}$, (c) Histogram of the sensitive responses at $230^{\circ} \mathrm{C}$ (Reproduced with the permission from Ref. [258]).

For $10 \mathrm{ppm} \mathrm{HCHO}$, the sensor response reaches 12.7 at low operating temperature $\left(139^{\circ} \mathrm{C}\right)$ with short response/recovery time $(4 \mathrm{~s} / 9 \mathrm{~s})$ and sub-ppm LOD $(0.3 \mathrm{ppm})$. Therefore, development of such nanocube materials are highly anticipated in $\mathrm{HCHO}$ quantitation. 
Nanosheets with and without decoration were effectively applied in the sensing of $\mathrm{HCHO}$. High resolution SEM and TEM images of nanosheets are dispayed in Figure $16 . \mathrm{WO}_{3}$ clusters decorated $\mathrm{In}_{2} \mathrm{O}_{3}$ NShs (synthesized by impregnating method), $\mathrm{SnO}_{2} \mathrm{NSh}$, ZnO NShs, $\mathrm{Au}$ atom dispersed $\mathrm{In}_{2} \mathrm{O}_{3}$ NShs, and PdAu bimetal decorated $\mathrm{SnO}_{2}$ NShs were exploited in the discrimination of $\mathrm{HCHO}$ at various operating temperatures [263-267]. However, the $\mathrm{ZnO}$ NShs (adopted from hydrothermal method; Figure 16) were engaged in aqueous phase detection of $\mathrm{HCHO}$ with linear regression of $10 \mathrm{nM}$ to $1 \mathrm{mM}$ and a LOD of $210 \mathrm{nM}$; thus, it cannot be listed as device-based assays [265]. Compared to other HCHO sensory reports, $\mathrm{Au}$ atom dispersed $\mathrm{In}_{2} \mathrm{O}_{3}$ NShs (developed by light assisted reduction method) were highly fascinated with respect to its sensor reposes $\left(R_{a} / R_{g}=85.67\right.$ for $50 \mathrm{ppm}$ at $100{ }^{\circ} \mathrm{C}$; response $/$ recovery time $=25 \mathrm{~s} / 198 \mathrm{~s}$ ) with an exceptional LOD of $0.00142 \mathrm{ppm}$ [266] $\mathrm{PdAu}$ bimetal decorated $\mathrm{SnO}_{2}$ NShs (synthesized by hydro-solvothermal treatment) were reported for the detection of both acetone and $\mathrm{HCHO}$ at $250{ }^{\circ} \mathrm{C}$ and $110^{\circ} \mathrm{C}$, respectively, with a LODs down to sub-ppm level [267]. The device also works at high humid condition ( $94 \% \mathrm{RH})$, but interference studies still need more clarification., Hayashi et al. proposed the utilization of $\mathrm{SnS}_{2}$ nanoflake device to detect the $\mathrm{HCHO}$ gas [268], which had a LOD down to sub-ppm $(0.001 / 0.02 \mathrm{ppm})$ and operated at $210^{\circ} \mathrm{C}$. However, details on other sensory properties are currently missing.

Nanospheres were also fabricated to quantify the $\mathrm{HCHO}$ as described in the following. Hussain and co-workers demonstrated the utilization of 0D ZnO NSPs and NPs (developed by low temperature hydrothermal route) to discriminate the formaldehyde [269]. Wherein, 0D ZnO NSPs displayed a higher sensor response $\left(\mathrm{R}_{\mathrm{a}} / \mathrm{R}_{\mathrm{g}}=95.4\right.$ for $100 \mathrm{ppm}$ at $295^{\circ} \mathrm{C}$; response $/$ recovery time $=11 \mathrm{~s} / 8 \mathrm{~s}$; LOD as $\sim 5 \mathrm{ppm})$ than that of $0 \mathrm{D} \mathrm{ZnO} \mathrm{NPs}\left(\mathrm{R}_{\mathrm{a}} / \mathrm{R}_{\mathrm{g}}=68.2\right.$ for $100 \mathrm{ppm}$ at $295^{\circ} \mathrm{C}$; response $/$ recovery time $=11 \mathrm{~s} / 8 \mathrm{~s}$; LOD $\left.=\sim 10 \mathrm{ppm}\right)$. However, the working temperature needs to be reduced before commercialization. In light of this, hydrothermally synthesized Ag-doped $\mathrm{Zn}_{2} \mathrm{SnO}_{4} / \mathrm{SnO}_{2}$ hollow NSPs [270] were reported in the detection of $\mathrm{HCHO}$ at low working temperature $\left(\mathrm{R}_{\mathrm{a}} / \mathrm{R}_{\mathrm{g}}=60\right.$ for $50 \mathrm{ppm}$ at $140{ }^{\circ} \mathrm{C}$; response/recovery time $=9 \mathrm{~s} / 5 \mathrm{~s} ; \mathrm{LOD}=5 \mathrm{ppm}$ ), thereby is attested as a better candidate. Similar to NSPs, microspheres were also still applied in the detection of HCHO. For example, MOF-derived $\mathrm{ZnO} / \mathrm{ZnCo}_{2} \mathrm{O}_{4}$ microspheres [271] were used in $\mathrm{HCHO}$ sensing with a response of 26.9 for $100 \mathrm{ppm}$ gas with response/recovery time of $9 \mathrm{~s} / 14 \mathrm{~s}$ and a LOD of $0.2 \mathrm{ppm}$. Therefore, research on NSPs/microspheres-based VOCs detection is highly anticipated in future.

As an alternative to $\mathrm{HCHO}$ sensors, materials with nanoflowers like structures were developed. Hierarchical $\mathrm{SnO}_{2}$ and $\mathrm{Sn}_{3} \mathrm{O}_{4} / \mathrm{rGO}$ hetrostructured nanoflowers (synthesized by hydrothermal method) were reported for formaldehyde assay [272,273]. As noted in Table $3, \mathrm{Sn}_{3} \mathrm{O}_{4} / \mathrm{rGO}$ hetrostructured nanoflowers are more effective in sensing $\mathrm{HCHO}$ $\left(\mathrm{R}_{\mathrm{a}} / \mathrm{R}_{\mathrm{g}}=44\right.$ for $100 \mathrm{ppm}$ at $150{ }^{\circ} \mathrm{C}$; response/recovery time $\left.=4 \mathrm{~s} / 125 \mathrm{~s}\right)$ than that of Hierarchical $\mathrm{SnO}_{2}$ nanoflowers with a LOD of $1 \mathrm{ppm}$. Moreover, the presence of rGO enhances the sensor response and long-term stability, thereby is noted as a good addition to $\mathrm{HCHO}$ sensors.

Exploitation of different nanostructures with porosity in the quantitation of $\mathrm{HCHO}$ detection has been demonstrated. Porous nanostructures such as Au-loaded $\operatorname{In}_{2} \mathrm{O}_{3}$ hierarchical porous nanocubes, Ag-loaded $\mathrm{ZnO}$ porous hierarchical nanocomposite, $\mathrm{Pd}-\mathrm{WO}_{3} / \mathrm{m}$ $\mathrm{CN}$ mesoporous nanocubes, $\mathrm{GO} / \mathrm{SnO}_{2}-2 \mathrm{D}$ mesoporous nanosheets, $\mathrm{ZnSnO} 3-2 \mathrm{D}$ mesoporous nanostructure, $\mathrm{LaFeO}_{3}$ porous hierarchical nanostructure, $\mathrm{Bi}$ doped $\mathrm{Zn}_{2} \mathrm{SnO}_{4} / \mathrm{SnO}_{2}$ porous nanospheres, $\mathrm{ZnO}$ porous nanoplates, and $\mathrm{Au} @ \mathrm{ZnO}$ mesoporous nanoflowers were reported as $\mathrm{HCHO}$ sensors at diverse operating temperatures as summarized in Table 3 [274-282]. The higher sensor responses achieved from those materials are attributed to the presence of porosity, which increases the adsorption of the $\mathrm{HCHO}$ gas significantly to enhance the signal (changes in resistance). Among the aforementioned porous materials, 2D mesoporous $\mathrm{GO} / \mathrm{SnO}_{2}$ nanosheets [277] revealed an exceptional sensor response of 2275.7 at low operating temperature $\left(60^{\circ} \mathrm{C}\right)$ with the response/recovery time of $81.3 \mathrm{~s} / 33.7 \mathrm{~s}$ and a LOD of $0.25 \mathrm{ppm}$. Therefore, this extraordinary work has demonstrated possible 
commercialization in future. Similarly, mesoporous $\mathrm{Pd}-\mathrm{WO}_{3} / \mathrm{m}-\mathrm{CN}$ nanocubes [276] performed quite impressively with operation at $95 \%$ dry humid condition. In the light of this, $\mathrm{TiO}_{2} / \mathrm{ZnCO}_{2} \mathrm{O}_{4}$ porous nanorods were engaged in the detection of $\mathrm{HCHO}$ and trimethylamine (TEA) at $220^{\circ} \mathrm{C}$ and $130{ }^{\circ} \mathrm{C}$ [283]. However, response in TEA detection was higher than that of $\mathrm{HCHO}$ and it was lack of interference studies.

(i)

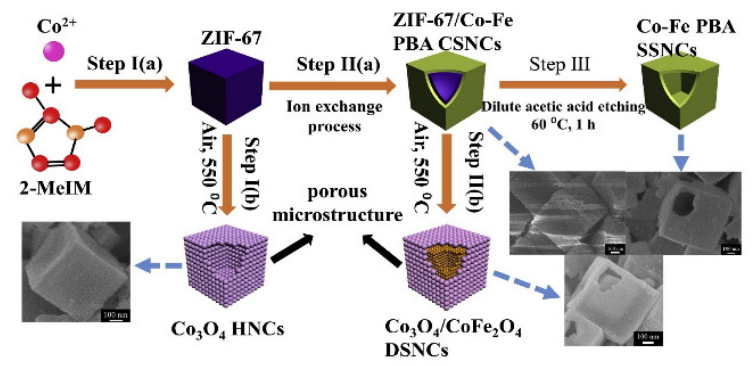

(ii)
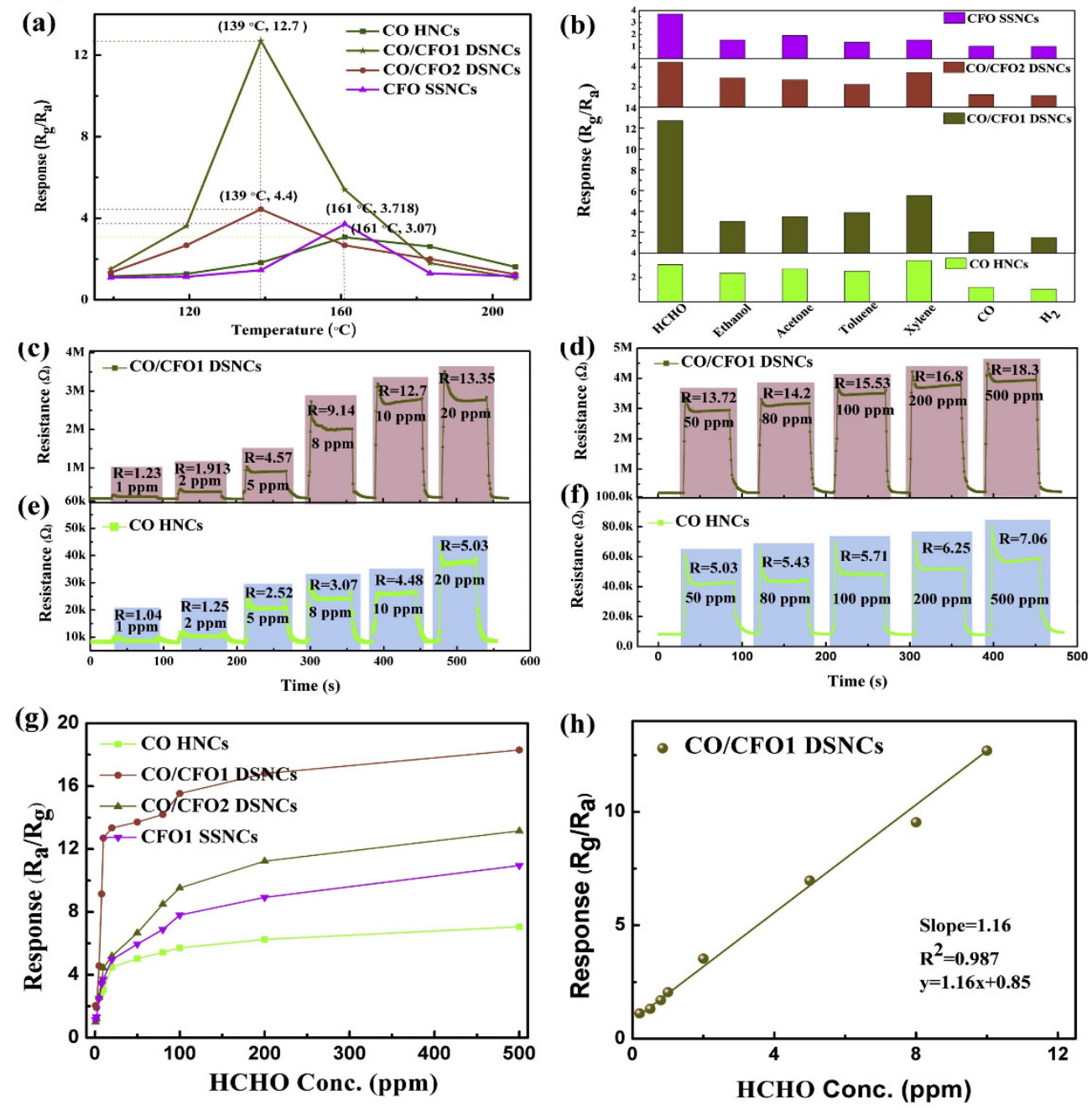

Figure 15. (i) Schematic of the process to fabricate porous $\mathrm{Co}_{3} \mathrm{O}_{4} \mathrm{HNCs}$, CCFO DSNCs, and CFO SSNCs; (ii) (a) Responses of the sensors to $10 \mathrm{ppm}$ of formaldehyde at $80-230^{\circ} \mathrm{C}$. (b) Responses of the sensors to $10 \mathrm{ppm}$ of various gases at the optimal operating temperatures. Dynamic responserecovery curves of the CCFO DSNCs and $\mathrm{Co}_{3} \mathrm{O}_{4} \mathrm{HNCs}$ sensors to xylene in the ranges (c,e) 1-20 ppm and $(\mathbf{d}, \mathbf{f})$ 50-500 ppm under their optimal operating temperatures. (g) Response of the sensors in the ranges from 1 to 500 ppm of xylene. (h) Responses of the CCFO DSNCs sensor as a function of low formaldehyde concentration (1-10 ppm) (Reproduced with the permission from Ref. [262]). 
Hierarchical nanostructures of $\mathrm{Zn}_{2} \mathrm{SnO}_{4} / \mathrm{SnO}_{2}, \mathrm{Pt} / \mathrm{MnO}_{2}-\mathrm{Ni}(\mathrm{OH})_{2}$ hybrid nanoflakes, $\mathrm{SnO}_{2}$ nanofiber/nanosheets, $\mathrm{In}_{2} \mathrm{O}_{3} @ \mathrm{SnO}_{2}$ composite, and cedar-like $\mathrm{SnO}_{2}$ were demonstrated as HCHO sensors [284-288]. These materials were synthesized by means of chemical route, hydrothermal, or electrospinning tactics. Wherein, hierarchical $\mathrm{Pt} / \mathrm{MnO}_{2}$ $\mathrm{Ni}(\mathrm{OH})_{2}$ hybrid nanoflakes [285] demonstrated only the formaldehyde oxidation activity at room temperature, thereby more work was needed to study the exact sensor response. Among these hierarchical nanostructures, $\mathrm{In}_{2} \mathrm{O}_{3} @ \mathrm{SnO}_{2}$ hierarchical composite displayed a good sensor response $\left(\mathrm{R}_{\mathrm{a}} / \mathrm{R}_{\mathrm{g}}=180.1\right.$ for $100 \mathrm{ppm}$ at $120^{\circ} \mathrm{C}$; response/recovery time $=$ $3 \mathrm{~s} / 3.6 \mathrm{~s}$ ) with a LOD of $0.1 \mathrm{ppm}$. Different shaped nanostructures were also proposed by researchers as an alternative to aldehyde sensors. Following the similar synthetic approaches, urchin-like $\mathrm{In}_{2} \mathrm{O}_{3}$ hollow nanostructure, butterfly-like $\mathrm{SnO}_{2}$ hierarchical nanostructure, $\mathrm{SnO}_{2}$ hollow hexagonal prisms, and $\mathrm{NiO} / \mathrm{NiFe}_{2} \mathrm{O}_{4}$ composite nanotetrahedrons were synthesized and used in aldehydes detection as illustrated in Table 3 [289-292]. However, butterfly-like $\mathrm{SnO}_{2}$ hierarchical nanostructure [290] performs better in the detection of acetaldehyde than that of $\mathrm{HCHO}$ as noted in Figure 17. It displays a high sensor response to acetaldehyde $\left(R_{a} / R_{g}=178.3\right.$ for $100 \mathrm{ppm}$ at $243{ }^{\circ} \mathrm{C}$; response/recovery time $=28 \mathrm{~s} / 58 \mathrm{~s}$ ) with a LOD of $<0.5 \mathrm{ppm}$, thereby cab be noted as an exceptional candidate. In the detection of $\mathrm{HCHO}$, nanocomposite materials were also effectively employed. Vertical graphene (VG) decorated $\mathrm{SnO}_{2}$, multiwalled carbon nanotubes-polyethyleneimine, and n-n $\mathrm{TiO}_{2} @ \mathrm{SnO}_{2}$ nanocomposites (synthesized by ALD method) were utilized in the discrimination of $\mathrm{HCHO}$ with LODs down to sub-ppm level [293-295]. In particular, multiwalled carbon nanotubes-polyethyleneimine composites [286] operated at room temperature with good response/recovery time ( $<1 \mathrm{~min}$ for both). On the other hand, n-n $\mathrm{TiO}_{2} @ \mathrm{SnO}_{2}$ nanocomposites [295] detected the $\mathrm{HCHO}$ under UV and dark conditions at $50{ }^{\circ} \mathrm{C}$. Therefore, development of such nanocomposite-based VOC sensor devices are much anticipated.

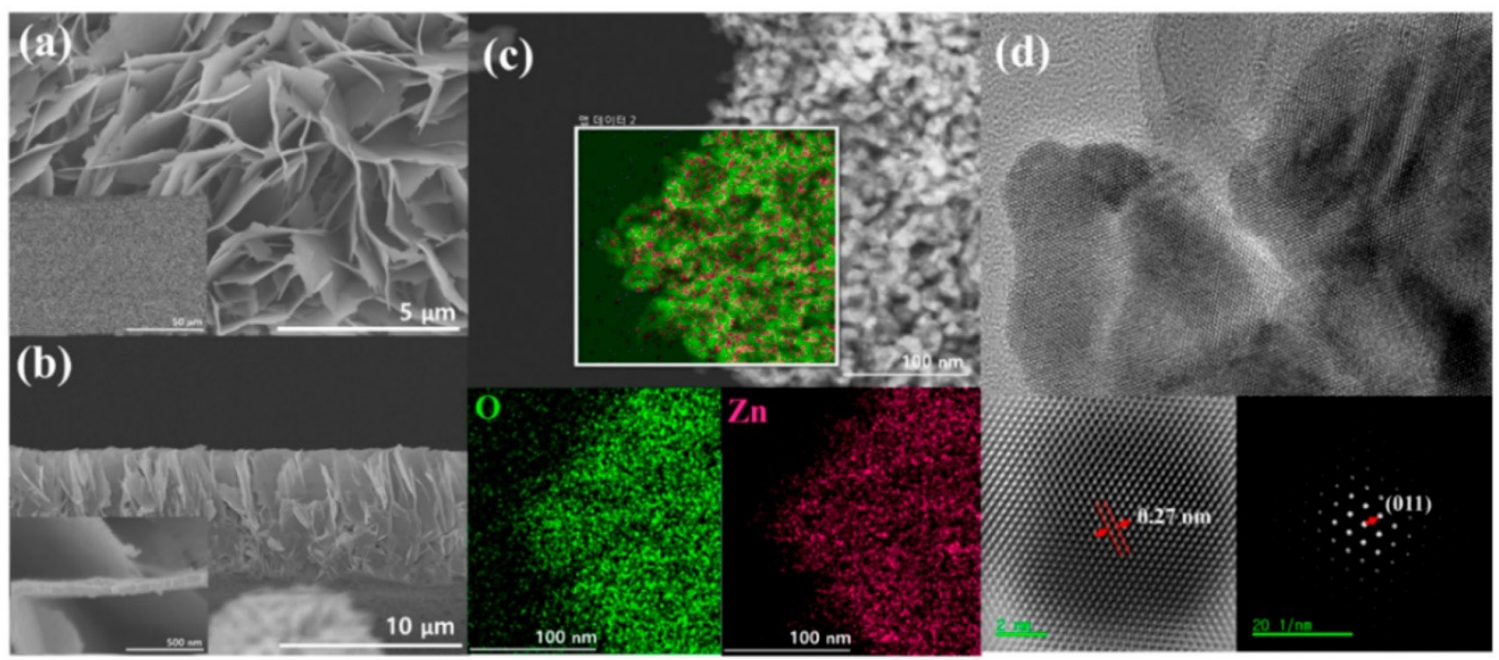

Figure 16. Field Emission Scanning Electron Microscopy (FESEM) image (a) cross section image (b), energy dispersive spectroscopy (EDS) mapping image (c) and transmission electron microscope (TEM) image (d) (inner HR TEM and selected area diffraction (SAED) pattern) of ZnO NShs (Reproduced with the permission from Ref. [265]). 


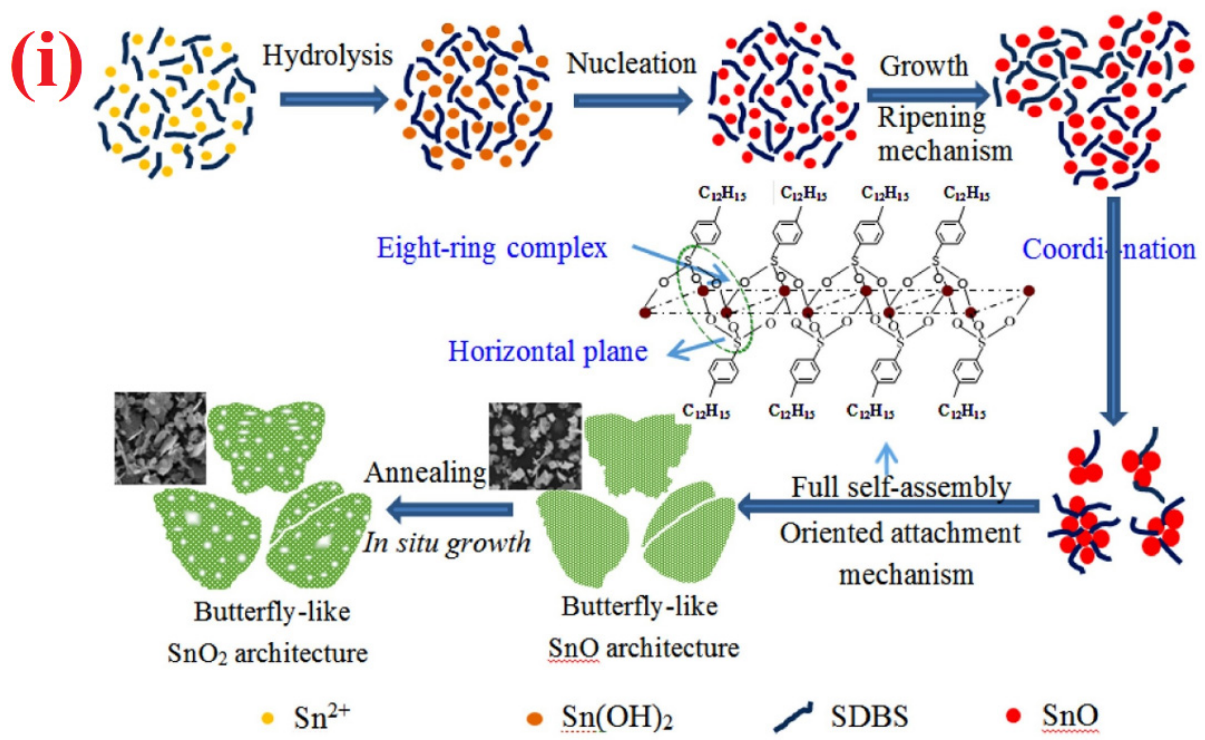

(ii)

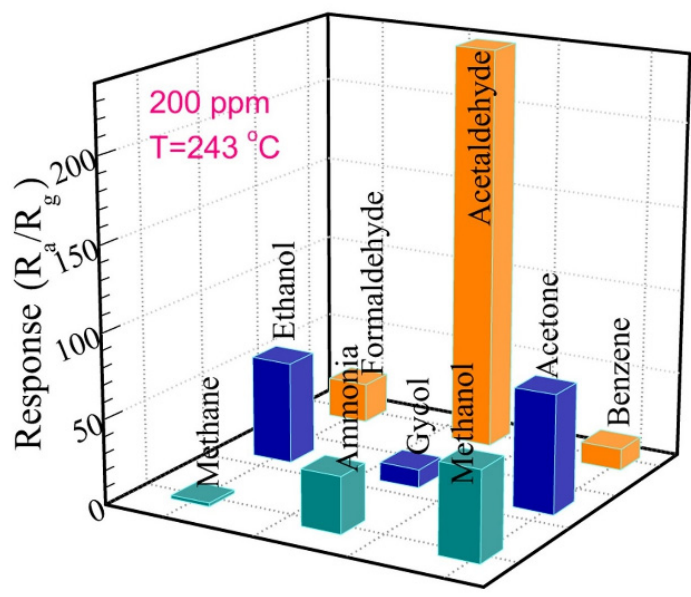

Figure 17. (i) Schematic illustration of the formation of butterfly-like $\mathrm{SnO}_{2}$ architectures. (ii) The response comparison of the butterfly-like $\mathrm{SnO}_{2}$ architectures to $200 \mathrm{ppm}$ of various VOCs at the optimal operating temperature. (Reproduced with the permission from Ref. [290]).

Table 3. Detection concentration, response/recovery time, operating temperature (Temp.) and LODs to volatile organic aldehyde gas by diverse nanostructured materials.

\begin{tabular}{|c|c|c|c|c|c|c|}
\hline Materials/Nanostructure & Analyte/Concentration & $\begin{array}{l}\text { Gas Response } \\
\left(\mathbf{R}_{\text {air }} / \mathbf{R}_{\text {gas }}\right)\end{array}$ & Response/Recovery & Temp. & LOD & Ref \\
\hline $\begin{array}{c}\mathrm{p}-\mathrm{CuO} / \mathrm{n}-\mathrm{SnO}_{2} / \text { core-shell } \\
\text { nanowires }\end{array}$ & Formaldehyde/50 ppm & 2.42 & $52 \mathrm{~s} / 80 \mathrm{~s}$ & $250^{\circ} \mathrm{C}$ & $<1.5 \mathrm{ppm}$ & [247] \\
\hline $\begin{array}{l}\mathrm{ZnO} / \text { meso-structured } \\
\text { nanowires (under UV) }\end{array}$ & Formaldehyde/50 ppm & $1223 \%$ & NA & $\begin{array}{l}\text { Room } \\
\text { Temp. }\end{array}$ & $0.005 \mathrm{ppm}$ & [248] \\
\hline RGO coated $\mathrm{Si} /$ nanowires & Formaldehyde/10 ppm & 6.4 & $30 \mathrm{~s} / 10 \mathrm{~s}$ & $300^{\circ} \mathrm{C}$ & $0.035 \mathrm{ppm}$ & [249] \\
\hline Co doped $\operatorname{In}_{2} \mathrm{O}_{3} /$ nanorods & Formaldehyde/10 ppm & 23.2 & $60 \mathrm{~s} / 120 \mathrm{~s}$ & $130^{\circ} \mathrm{C}$ & $1 \mathrm{ppm}$ & [250] \\
\hline $\begin{array}{l}\text { Ag-functionalized Ni-doped } \\
\operatorname{In}_{2} \mathrm{O}_{3} / \text { nanorods }\end{array}$ & Formaldehyde/100 ppm & 123.97 & $1.45 \mathrm{~s} / 58.2 \mathrm{~s}$ & $160^{\circ} \mathrm{C}$ & $<2.5 \mathrm{ppm}$ & [251] \\
\hline $\begin{array}{c}\mathrm{Ag} \text { doped } \\
\mathrm{LaFeO}_{3} / \text { nanofibers }\end{array}$ & Formaldehyde/5 ppm & 4.8 & $2 \mathrm{~s} / 4 \mathrm{~s}$ & $230^{\circ} \mathrm{C}$ & $\sim 5$ ppm & [253] \\
\hline $\begin{array}{c}\mathrm{Co}_{3} \mathrm{O}_{4}-\mathrm{ZnO} / \text { core-shell } \\
\text { nanofibers }\end{array}$ & Formaldehyde/100 ppm & $>5$ & $6 \mathrm{~s} / 9 \mathrm{~s}$ & $220^{\circ} \mathrm{C}$ & $<10 \mathrm{ppm}$ & [254] \\
\hline $\mathrm{WO}_{3} / \mathrm{ZnWO}_{4} /$ nanofibers & Formaldehyde $/ 5$ ppm & 44.5 & $12 \mathrm{~s} / 14 \mathrm{~s}$ & $220^{\circ} \mathrm{C}$ & $1 \mathrm{ppm}$ & [255] \\
\hline
\end{tabular}


Table 3. Cont.

\begin{tabular}{|c|c|c|c|c|c|c|}
\hline Materials/Nanostructure & Analyte/Concentration & $\begin{array}{l}\text { Gas Response } \\
\left(\mathbf{R}_{\text {air }} / \mathbf{R}_{\text {gas }}\right)\end{array}$ & Response/Recovery & Temp. & LOD & Ref \\
\hline $\begin{array}{l}\text { Pr-doped } \mathrm{BiFeO}_{3 / \text { hollow }} \\
\text { nanofibers }\end{array}$ & Formaldehyde/50 ppm & 17.6 & $17 \mathrm{~s} / 19 \mathrm{~s}$ & $190^{\circ} \mathrm{C}$ & $5 \mathrm{ppm}$ & [256] \\
\hline Ca doped $\operatorname{In}_{2} \mathrm{O}_{3} /$ nanotubes & Formaldehyde/100 ppm & 116 & $1 \mathrm{~s} / 328 \mathrm{~s}$ & $130^{\circ} \mathrm{C}$ & $0.06 \mathrm{ppm}$ & [257] \\
\hline Er-doped $\mathrm{SnO}_{2} /$ nanobelt & Formaldehyde/100 ppm & 9 & $17 \mathrm{~s} / 25 \mathrm{~s}$ & $230^{\circ} \mathrm{C}$ & $0.141 \mathrm{ppm}$ & [258] \\
\hline $\begin{array}{c}\text { Pt-decorated } \\
\mathrm{MoO}_{3} / \text { nanobelt }\end{array}$ & Formaldehyde/100 ppm & $\sim 25 \%$ & $17.8 \mathrm{~s} / 10.5 \mathrm{~s}$ & $\begin{array}{l}\text { Room } \\
\text { Temp. }\end{array}$ & $1 \mathrm{ppm}$ & [259] \\
\hline $\begin{array}{c}\mathrm{ZnSnO}_{3} / \text { multi-shelled } \\
\text { nanocubes }\end{array}$ & Formaldehyde/100 ppm & 37.2 & $1 \mathrm{~s} / 59 \mathrm{~s}$ & $220^{\circ} \mathrm{C}$ & $<10 \mathrm{ppm}$ & [260] \\
\hline $\begin{array}{l}\mathrm{ZnSn}(\mathrm{OH})_{6} / \text { multi-shelled } \\
\text { nanocubes }\end{array}$ & Formaldehyde/100 ppm & 56.6 & $1 \mathrm{~s} / 89 \mathrm{~s}$ & $60^{\circ} \mathrm{C}$ & $1 \mathrm{ppm}$ & [261] \\
\hline $\begin{array}{c}\text { MOF-derived } \\
\mathrm{Co}_{3} \mathrm{O}_{4} / \mathrm{CoFeO}_{4} / \text { double- } \\
\text { shelled } \\
\text { nanocubes }\end{array}$ & Formaldehyde/10 ppm & 12.7 & $4 \mathrm{~s} / 9 \mathrm{~s}$ & $139^{\circ} \mathrm{C}$ & $0.3 \mathrm{ppm}$ & [262] \\
\hline $\begin{array}{l}\mathrm{WO}_{\mathrm{x}} \text { clusters decorated } \\
\mathrm{In}_{2} \mathrm{O}_{3} / \text { nanosheets }\end{array}$ & Formaldehyde/100 ppm & $\sim 25$ & $1 \mathrm{~s} / 67 \mathrm{~s}$ & $170{ }^{\circ} \mathrm{C}$ & $0.1 \mathrm{ppm}$ & [263] \\
\hline $\mathrm{SnO}_{2} /$ nanosheets & Formaldehyde/200 ppm & 207.7 & $30 \mathrm{~s} / 57 \mathrm{~s}$ & $200^{\circ} \mathrm{C}$ & $0.1 \mathrm{ppm}$ & [264] \\
\hline $\begin{array}{l}\mathrm{Au} \text { atom dispersed } \\
\mathrm{In}_{2} \mathrm{O}_{3 /} \text { nanosheets }\end{array}$ & Formaldehyde/50 ppm & 85.67 & $25 \mathrm{~s} / 198 \mathrm{~s}$ & $100^{\circ} \mathrm{C}$ & $0.00142 \mathrm{ppm}$ & [266] \\
\hline $\mathrm{SnS}_{2 / \text { nanoflakes film }}$ & Formaldehyde/NA & NA & NA & $210^{\circ} \mathrm{C}$ & $0.001 / 0.02 \mathrm{ppm}$ & [268] \\
\hline $\begin{array}{l}\text { 0D ZnS/nanospheres and } \\
\text { nanoparticles }\end{array}$ & Formaldehyde/100 ppm & $95.4 \& 68.2$ & $11 \mathrm{~s} / 8 \mathrm{~s}$ & $295^{\circ} \mathrm{C}$ & $\sim 5 \& 10 \mathrm{ppm}$ & [269] \\
\hline $\begin{array}{c}\text { Ag doped } \\
\mathrm{Zn}_{2} \mathrm{SnO}_{4} / \mathrm{SnO}_{2 / \text { hollow }} \\
\text { nanospheres }\end{array}$ & Formaldehyde/50 ppm & 60 & $9 \mathrm{~s} / 5 \mathrm{~s}$ & $140^{\circ} \mathrm{C}$ & 5 ppm & [270] \\
\hline $\begin{array}{c}\mathrm{SnO}_{2} / \text { nanoflowers } \\
\text { (hierarchical) }\end{array}$ & Formaldehyde/100 ppm & 34.6 & $64 \mathrm{~s} / 10 \mathrm{~s}$ & $300^{\circ} \mathrm{C}$ & $5 \mathrm{ppm}$ & [272] \\
\hline $\begin{array}{l}\mathrm{Sn}_{3} \mathrm{O}_{4} / \mathrm{rGO} / \text { nanoflower } \\
\text { (hetero- structure) }\end{array}$ & Formaldehyde/100 ppm & 44 & $4 \mathrm{~s} / 125 \mathrm{~s}$ & $150^{\circ} \mathrm{C}$ & $1 \mathrm{ppm}$ & [273] \\
\hline $\begin{array}{l}\text { Au-loaded } \operatorname{In}_{2} \mathrm{O}_{3} / \text { porous } \\
\text { hierarchical nanocubes }\end{array}$ & Formaldehyde/100 ppm & 37 & $3 \mathrm{~s} / 8 \mathrm{~s}$ & $240^{\circ} \mathrm{C}$ & $10 \mathrm{ppm}$ & [274] \\
\hline $\begin{array}{l}\text { Ag-loaded } \mathrm{ZnO} / \text { porous } \\
\text { hierarchical nano- } \\
\text { composite }\end{array}$ & Formaldehyde/100 ppm & 170.42 & $12 \mathrm{~s} / 90 \mathrm{~s}$ & $240^{\circ} \mathrm{C}$ & $1 \mathrm{ppm}$ & [275] \\
\hline $\begin{array}{c}\mathrm{Pd}-\mathrm{WO}_{3} / \mathrm{m}- \\
\mathrm{CN} / \text { mesoporous } \\
\text { nanocubes }\end{array}$ & Formaldehyde/25 ppm & 24.2 & $6.8 \mathrm{~s} / 4.5 \mathrm{~s}$ & $120^{\circ} \mathrm{C}$ & $1 \mathrm{ppm}$ & [276] \\
\hline $\begin{array}{c}\mathrm{GO} / \mathrm{SnO}_{2} / 2 \mathrm{D} \text { mesoporous } \\
\text { nanosheets }\end{array}$ & Formaldehyde/100 ppm & 2275.7 & $81.3 \mathrm{~s} / 33.7 \mathrm{~s}$ & $60^{\circ} \mathrm{C}$ & $0.25 \mathrm{ppm}$ & [277] \\
\hline $\begin{array}{c}\mathrm{ZnSnO}_{3 / 2 \mathrm{D}} \text { mesoporous } \\
\text { nanostructure }\end{array}$ & Formaldehyde/100 ppm & 45.8 & $3 \mathrm{~s} / 6 \mathrm{~s}$ & $210^{\circ} \mathrm{C}$ & $0.2 \mathrm{ppm}$ & [278] \\
\hline $\begin{array}{c}\mathrm{LaFeO}_{3} / \text { porous hierarchical } \\
\text { nanostructure }\end{array}$ & Formaldehyde/50 ppm & 116 & $7 \mathrm{~s} / 24 \mathrm{~s}$ & $125^{\circ} \mathrm{C}$ & $0.05 \mathrm{ppm}$ & [279] \\
\hline $\begin{array}{c}\text { Bi doped } \\
\mathrm{Zn}_{2} \mathrm{SnO}_{4} / \mathrm{SnO}_{2} / \text { porous } \\
\text { nanospheres }\end{array}$ & Formaldehyde/50 ppm & 23.2 & $16 \mathrm{~s} / 9 \mathrm{~s}$ & $180^{\circ} \mathrm{C}$ & $10 \mathrm{ppm}$ & [280] \\
\hline $\mathrm{ZnO} /$ porous nanoplates & Formaldehyde/100 ppm & 12 & $80 \mathrm{~s} / 60 \mathrm{~s}$ & $240^{\circ} \mathrm{C}$ & $10 \mathrm{ppm}$ & [281] \\
\hline $\begin{array}{c}\mathrm{Au} @ \mathrm{ZnO} / \text { mesoporous } \\
\text { nanoflowers }\end{array}$ & Formaldehyde/100 ppm & 45.28 & NA & $220^{\circ} \mathrm{C}$ & NA & [282] \\
\hline $\begin{array}{c}\mathrm{Zn}_{2} \mathrm{SnO}_{4} / \mathrm{SnO}_{2} / \text { hierarchical } \\
\text { octahedral nanostructure }\end{array}$ & Formaldehyde/100 ppm & $>60$ & $\begin{array}{l}76 \mathrm{~s} / 139 \mathrm{~s}(\text { for } 20 \\
\text { ppm) }\end{array}$ & $200^{\circ} \mathrm{C}$ & $2 \mathrm{ppm}$ & [284] \\
\hline $\begin{array}{l}\mathrm{SnO}_{2} \text { nanofiber/nanosheet/ } \\
\text { hierarchical nanostructure }\end{array}$ & Formaldehyde/100 ppm & 57 & $4.7 \mathrm{~s} / 11.6 \mathrm{~s}$ & $120^{\circ} \mathrm{C}$ & $\sim 0.5 \mathrm{ppm}$ & [286] \\
\hline
\end{tabular}


Table 3. Cont.

\begin{tabular}{|c|c|c|c|c|c|c|}
\hline Materials/Nanostructure & Analyte/Concentration & $\begin{array}{c}\text { Gas Response } \\
\left(\mathbf{R}_{\text {air }} / \mathbf{R}_{\text {gas }}\right)\end{array}$ & Response/Recovery & Temp. & LOD & Ref \\
\hline $\begin{array}{c}\mathrm{In}_{2} \mathrm{O}_{3} @ \mathrm{SnO}_{2} / \text { hierarchical } \\
\text { nano- composite }\end{array}$ & Formaldehyde/100 ppm & 180.1 & $3 \mathrm{~s} / 3.6 \mathrm{~s}$ & $120^{\circ} \mathrm{C}$ & $0.01 \mathrm{ppm}$ & [287] \\
\hline $\begin{array}{l}\mathrm{SnO}_{2} / \text { cedar like hierarchical } \\
\text { nano-micro structure }\end{array}$ & Formaldehyde/100 ppm & 13.3 & $<1 \mathrm{~s} / 13 \mathrm{~s}$ & $200^{\circ} \mathrm{C}$ & $\sim 5 \mathrm{ppm}$ & [288] \\
\hline $\begin{array}{l}\mathrm{In}_{2} \mathrm{O}_{3} / \text { urchin like hollow } \\
\text { nanostructure }\end{array}$ & Formaldehyde/1 ppm & 20.9 & $46 \mathrm{~s} / 90 \mathrm{~s}$ & $140^{\circ} \mathrm{C}$ & $0.05 \mathrm{ppm}$ & [289] \\
\hline $\begin{array}{c}\mathrm{SnO}_{2} / \text { Butterfly like } \\
\text { hierarchical nanostructure }\end{array}$ & Acetaldehyde/100 ppm & 178.3 & $28 \mathrm{~s} / 58 \mathrm{~s}$ & $243^{\circ} \mathrm{C}$ & $<0.5 \mathrm{ppm}$ & [290] \\
\hline $\begin{array}{l}\mathrm{SnO}_{2} / \text { hollow hexagonal } \\
\text { prisms }\end{array}$ & Formaldehyde/2 ppm & 882 & $19 \mathrm{~s} / \mathrm{NA}$ & $120^{\circ} \mathrm{C}$ & $<2 \mathrm{ppm}$ & [291] \\
\hline $\begin{array}{l}\mathrm{NiO} / \mathrm{NiFe}_{2} \mathrm{O}_{4} / \text { nano- } \\
\text { tetrahedrons composite }\end{array}$ & Formaldehyde/100 ppm & 22.5 & $9 \mathrm{~s} / 3 \mathrm{~s}$ & $240^{\circ} \mathrm{C}$ & $0.2 \mathrm{ppm}$ & [292] \\
\hline $\mathrm{VG} / \mathrm{SnO}_{2} /$ nano- composite & Formaldehyde/5 ppm & $>5$ & $46 \mathrm{~s} / 95 \mathrm{~s}$ & $\begin{array}{l}\text { Room } \\
\text { Temp. }\end{array}$ & $0.02 \mathrm{ppm}$ & [293] \\
\hline
\end{tabular}

NA = Not available; Temp. = Temperature; $\mathrm{s}=$ seconds; $\mathrm{min}=$ minutes .

\section{Various Nanostructures in Volatile Organic Amines Detection}

The well-known toxic volatile organic amines detection by nanomaterials are highly anticipated. The mechanism follows the similar trend as proposed in other VOCs sensors. Upon interaction with anionic oxide species in the chamber, the volatile amines reacts and releases electrons resulting changes in resistance, which is adopted as sensor signals. A simple scheme represents the reaction mechanisms in triethylamine (TEA) detection is given in Equations (10)-(12). Similar to this mechanism, other volatile organic amine detections also follow the same sequence.

$$
\begin{gathered}
\text { In chamber } \mathrm{O}_{2} \text { (ads) }+\mathrm{e}^{-} \rightarrow \mathrm{O}_{2}{ }^{-} \text {(ads) } \\
\text { In chamber } \mathrm{O}_{2}{ }^{-} \text {(ads) }+\mathrm{e}^{-} \rightarrow 2 \mathrm{O}^{-} \text {(ads) } \\
\text { Under TEA }\left(\mathrm{C}_{2} \mathrm{H}_{5}\right)_{3} \mathrm{~N}+\mathrm{O}^{-} \text {(ads) } \rightarrow \mathrm{N}_{2}+\mathrm{CO}_{2}+\mathrm{H}_{2} \mathrm{O}+\mathrm{e}^{-}
\end{gathered}
$$

TEA detection was demonstrated by nanoparticle-based devices as explained below. $\mathrm{Co}_{3} \mathrm{O}_{4} / \mathrm{ZnO}$ hybrid NPs, Ho-doped $\mathrm{SnO}_{2} \mathrm{NPs}$, and $\mathrm{CuCrO}_{2} \mathrm{NPs}$ (synthesized by hydrothermal or gas-liquid phase chemical method and annealing) were utilized in the sensing of TEA at $285^{\circ} \mathrm{C}, 175^{\circ} \mathrm{C}$, and $140^{\circ} \mathrm{C}$, respectively, as shown in Table 4 [296-298]. Wherein, $\mathrm{Co}_{3} \mathrm{O}_{4} / \mathrm{ZnO}$ hybrid NPs [296] shows a high response $\left(\mathrm{R}_{\mathrm{a}} / \mathrm{R}_{\mathrm{g}}=282.3\right.$ for 200 ppm at $285{ }^{\circ} \mathrm{C}$; response/recovery time $=25 \mathrm{~s} / 36 \mathrm{~s}$ ) with a LOD of $\sim 10 \mathrm{ppm}$. However, more investigations are needed to lower the working temperature. Subsequently, multimetal functionalized tungsten oxide $\mathrm{NWs}\left(\mathrm{Ag} / \mathrm{Pt} / \mathrm{W}_{18} \mathrm{O}_{49} \mathrm{NWs}\right)$ and $1 \mathrm{D} \mathrm{SnO}_{2}$ coated $\mathrm{ZnO}$ hybrid NWs were reported in the discrimination of TEA and n-Butylamine, correspondingly, at $240{ }^{\circ} \mathrm{C}$. The $\mathrm{Ag} / \mathrm{Pt} / \mathrm{W}_{18} \mathrm{O}_{49} \mathrm{NWs}$ [299] displayed a high sensor response to TEA $\left(\mathrm{R}_{\mathrm{a}} / \mathrm{R}_{\mathrm{g}}=813\right.$ for $50 \mathrm{ppm}$ at $240^{\circ} \mathrm{C}$; response/recovery time $=15 \mathrm{~s} / 35 \mathrm{~s}$ (for $\left.2 \mathrm{ppm}\right)$ with a LOD of $0.071 \mathrm{ppm}$, thereby is noted as a remarkable candidate. $1 \mathrm{D}^{\mathrm{SnO}_{2}}$ coated $\mathrm{ZnO}$ hybrid NWs (synthesized by solvothermal and calcination tactics) revealed sensitivity to n-Butylamine $\left(R_{a} / R_{g}=7.4\right.$ for $10 \mathrm{ppm}$ at $240{ }^{\circ} \mathrm{C}$; response/recovery time is $\left.40 \mathrm{~s} / 80 \mathrm{~s}\right)$ with an estimated LOD of 1 ppm, thereby can be included in n-Butylamine detection [300].

Apart from NWs, nanorods were also engaged in the quantitation of volatile organic amines as detailed in the following. The sensing of Diethylamine (DEA), Trimethylamine (TMA), and TEA were demonstrated by $\mathrm{V}_{2} \mathrm{O}_{5}$-decorated $\alpha-\mathrm{Fe}_{2} \mathrm{O}_{3}$ NRds, Au NPs decorated $\mathrm{WO}_{3} \mathrm{NRds}$, Ag NPs decorated $\alpha-\mathrm{MoO}_{3} \mathrm{NRds}, \mathrm{Cr}$ doped $\alpha-\mathrm{MoO}_{3} \mathrm{NRds}$, acidic $\alpha-\mathrm{MoO}_{3} \mathrm{NRds}$, and $\mathrm{NiCo}_{2} \mathrm{O}_{4}$ microspheres assembled by hierarchical NRds as illustrated in Table 4 [301-306]. All these NRds were synthesized through electrospinning, calcination, wet-chemical reduction, thermal annealing, or by hydrothermal methods. Wherein, $\mathrm{V}_{2} \mathrm{O}_{5}$ - 
decorated $\alpha-\mathrm{Fe}_{2} \mathrm{O}_{3}$ NRds and $\mathrm{Au}$ NPs decorated $\mathrm{WO}_{3}$ NRds $[293,294]$ demonstrated sensor utility towards DEA and TMA with the responses of 8.9 and 76.7 (response/recovery time $=2 \mathrm{~s} / 40 \mathrm{~s}$ and $6 \mathrm{~s} / 7 \mathrm{~s}$, respectively) at $350{ }^{\circ} \mathrm{C}$ and $280^{\circ} \mathrm{C}$, respectively. The LODs of DEA and TMA are estimated as $\sim 5 \mathrm{ppm}$, apart from the working temperature, it is worthy of continuing research. All other NRds [303-306] can detect the TEA at various operating temperatures (between 180 and $300{ }^{\circ} \mathrm{C}$ ) as revealed in Table 4. Among them, Ag NPs decorated $\alpha-\mathrm{MoO}_{3} \mathrm{NRds}$ [295] show an exceptional sensor response to TEA $\left(\mathrm{R}_{\mathrm{a}} / \mathrm{R}_{\mathrm{g}}=408.6\right.$ for $100 \mathrm{ppm}$ at $200{ }^{\circ} \mathrm{C}$; response/recovery time $=3 \mathrm{~s} / 107 \mathrm{~s}$ ) with a LOD of $0.035 \mathrm{ppm}$. On the other hand, $\mathrm{NiCO}_{2} \mathrm{O}_{4}$ microspheres assembled by hierarchical $\mathrm{NRds}$ [306] has a low sensor response to TEA $\left(\mathrm{R}_{\mathrm{a}} / \mathrm{R}_{\mathrm{g}} \leq 1.5\right.$ for $50 \mathrm{ppm}$ at $180{ }^{\circ} \mathrm{C}$; response/recovery time $=$ $49 \mathrm{~s} / 54 \mathrm{~s}$ ) with a LOD of $0.145 \mathrm{ppm}$, hence further investigations are mandatory on this material to improve the response.

Towards enhanced sensing of TEA, Xu and co-workers fabricated the $\mathrm{Au} @ \mathrm{SnO}_{2} / \alpha-$ $\mathrm{Fe}_{2} \mathrm{O}_{3}$ core-shell nanoneedles directly on alumina tubes via pulsed laser deposition (PLD) and DC-sputtering methods [307]. As shown in Figure 18, Au@SnO $2 / \alpha-\mathrm{Fe}_{2} \mathrm{O}_{3}$ core-shell nanoneedles displayed a higher response to TEA $\left(\mathrm{R}_{\mathrm{a}} / \mathrm{R}_{\mathrm{g}}=39\right.$ for $100 \mathrm{ppm}$ at $300{ }^{\circ} \mathrm{C}$; response $/$ recovery time $=4 \mathrm{~s} / 203 \mathrm{~s}$ ) than that of $\alpha-\mathrm{Fe}_{2} \mathrm{O}_{3}$ nanoneedles and $\mathrm{SnO}_{2} / \alpha-\mathrm{Fe}_{2} \mathrm{O}_{3}$ core-shell nanoneedles with a LOD of $\sim 2$ ppm. However, more interrogations are required to increase the response and to reduce the operating temperature.

(i)

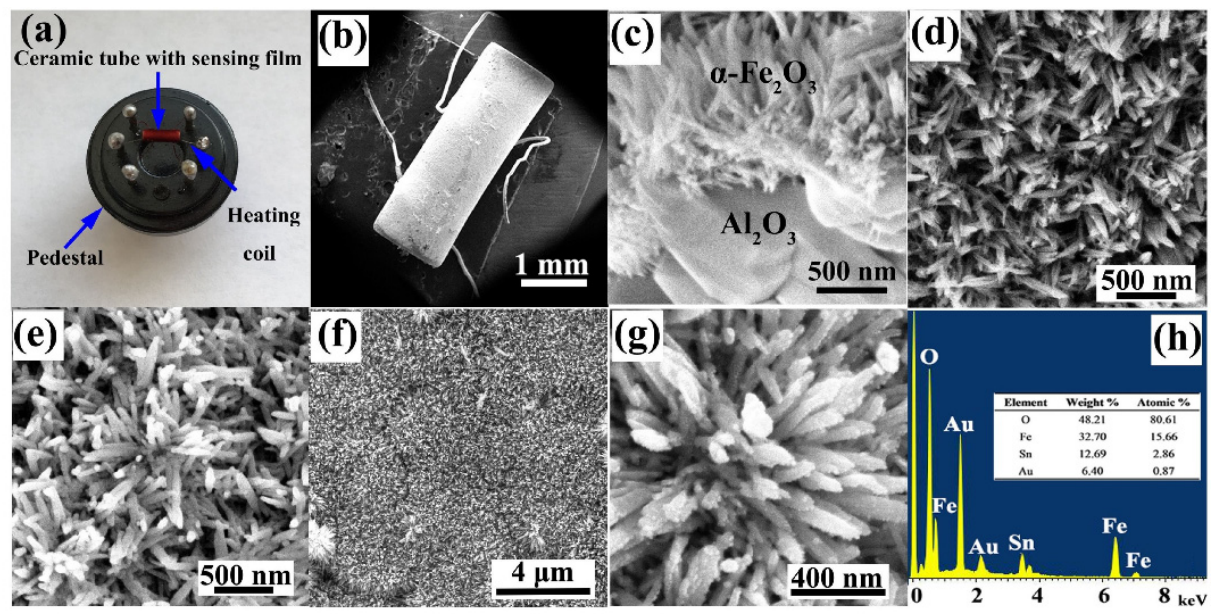

(ii)

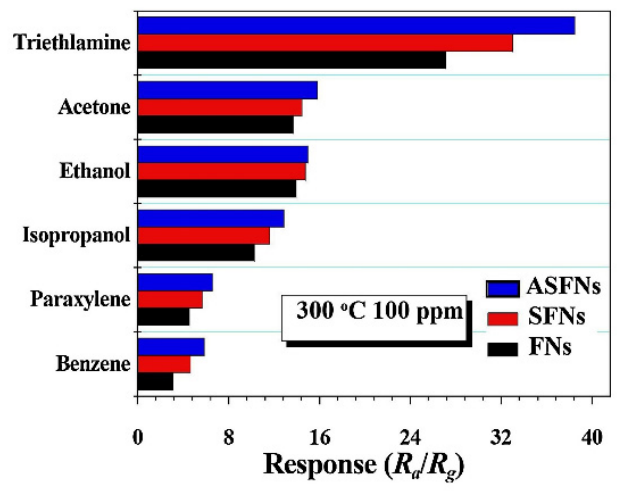

Figure 18. (i) (a) Gas sensor of $\alpha-\mathrm{Fe}_{2} \mathrm{O}_{3}$ nanosheets fixed on an electronic bracket (b) $\mathrm{Al}_{2} \mathrm{O}_{3}$ cube covered with a film of sensing materials; (c) SEM image of $\mathrm{SnO}_{2}$ nanosheet in cross-sectional view; (d) SEM image of $\alpha-\mathrm{Fe}_{2} \mathrm{O}_{3}$ nanoneedles (FNs) directly grown on $\mathrm{Al}_{2} \mathrm{O}_{3}$ tubes; (e) SEM images of SFNs; (f,g) SEM images of $\mathrm{Au} @ \mathrm{SnO}_{2} / \alpha-\mathrm{Fe}_{2} \mathrm{O}_{3}$ nanoneedles (ASFNS) after implantation of $\mathrm{SnO}_{2}$ shell and Au nanoparticles; (h) EDS spectrum of ASFNs; (ii) The selectivity of different gases with same concentration at $300{ }^{\circ} \mathrm{C}$ (Reproduced with the permission from Ref. [307]). 
By means of calcination and electrospinning, nanofibers $\left(\mathrm{Al}_{2} \mathrm{O}_{3} / \alpha-\mathrm{Fe}_{2} \mathrm{O}_{3}\right.$ nanofibers and $\operatorname{In}_{2} \mathrm{O}_{3}$ hierarchical nanofibers with in situ growth of octahedron particles) were proposed by the researchers for TEA sensing studies [308,309]. As depicted in Figure 19, the $\mathrm{Al}_{2} \mathrm{O}_{3} / \alpha-\mathrm{Fe}_{2} \mathrm{O}_{3}$ nanofibers [308] evidence the sensor signal even at $0.5 \mathrm{ppm}$. The sensor response to TEA is 15.19 (for $100 \mathrm{ppm}$ ) at $250{ }^{\circ} \mathrm{C}$ (response/recovery time $=1 \mathrm{~s} / 17 \mathrm{~s}$ ), but it is important to further reduce the working temperature in this report. In contrast, $\mathrm{In}_{2} \mathrm{O}_{3}$ hierarchical nanofibers with in situ growth of octahedron particles [309] revealed the highest response to TEA at $40{ }^{\circ} \mathrm{C}\left(\mathrm{R}_{\mathrm{a}} / \mathrm{R}_{\mathrm{g}}=87.8\right.$ for $50 \mathrm{ppm}$; response $/$ recovery time $=$ $148 \mathrm{~s} / 40 \mathrm{~min}$ ) with a LOD of $\sim 5 \mathrm{ppm}$, thereby is noted as an excellent work. Subsequently, $\mathrm{TiO}_{2}$ membrane NTs and sidewall modified single-walled carbon nanotubes (SWCNTs) were employed in the detection of TMA vapor [310,311]. The flexible $\mathrm{TiO}_{2}$ membrane NTs showed a response of 40 for $400 \mathrm{ppm}$ of TMA, but details regarding the working temperature and response/recovery time were not clear. Similarly, sidewall modified SWCNTs were demonstrated with selectivity to both ammonia and TMA at room temperature, thereby cannot be stated as a successful work. In view of this, Galstyan et al. described the DMA sensing utility of $\mathrm{Nb}$ doped $\mathrm{TiO}_{2}$ nanotubes at $300{ }^{\circ} \mathrm{C}$ as illustrated in Table 4 [312]. This work requires additional interrogations to attain a high response at low temperature.

Nanobelts such as $\mathrm{Au}$ NPs decorated $\mathrm{MoO}_{3}$ nanobelts, $\mathrm{W}$ doped $\mathrm{MoO}_{3}$ nanobelts, $\mathrm{RuO}_{2} \mathrm{NPs}$ decorated $\mathrm{MoO}_{3}$ nanobelts, and $\mathrm{ZnO}-\mathrm{SnO}_{2}$ nanobelts were developed by hydrothermal, soaking, and two step synthesis, etc., and applied in the discrimination of TMA and TEA [313-316]. Doping and decoration are the two important steps to enhance the senor signals. As shown in Table 4, these nanobelts, except the $\mathrm{RuO}_{2} \mathrm{NPs}$ decorated $\mathrm{MoO}_{3}$ nanobelts, used in the TMA and TEA sensing interrogations require more efforts to enhance the response and to minimize the operating temperature. The $\mathrm{RuO}_{2} \mathrm{NPs}$ decorated $\mathrm{MoO}_{3}$ nanobelts evidenced a good response $\left(R_{a} / R_{g}=75\right.$ for $10 \mathrm{ppm}$ at $260^{\circ} \mathrm{C}$; response/recovery time $=2 \mathrm{~s} / 10 \mathrm{~s}$ ) with a LOD of $\sim 1 \mathrm{ppm}$, but the working temperature still needs further optimization. Towards TEA detection, Zhang and co-workers described the utilization of hydrothermally synthesized $\operatorname{In}_{2} \mathrm{O}_{3}$ nanocubes [317], which showed a high response $\left(\mathrm{R}_{\mathrm{a}} / \mathrm{R}_{\mathrm{g}}\right.$ $=175$ for $100 \mathrm{ppm}$ at $260^{\circ} \mathrm{C}$; response/recovery time $=11 \mathrm{~s} / 14 \mathrm{~s}$ ) with a LOD of $\sim 10 \mathrm{ppm}$, thereby is noted as an encouraging research.

As an important candidate in the TEA sensory studies, nanosheets were intensively studied by many research groups. $\mathrm{WO}_{3} \mathrm{NShs}$, Au@ZnO-SnO $\mathrm{NSh}_{2}, \mathrm{TiO}_{2} \mathrm{NPs}$ decorated $\mathrm{CuO}$ NShs, and $\mathrm{Rh}-\mathrm{SnO}_{2}$ NShs were synthesized by precipitation, $\mathrm{PLD}$, water bath treatment and solution etching methods, and surface impregnation precipitation and heat treatment method, respectively, and applied in TEA detection [318-321]. Among them, $\mathrm{WO}_{3} \mathrm{NShs}$ operate at room temperature and show a sensor response of $\sim 14$ for $1000 \mathrm{ppm}$ as shown in Table 4. Though this work is an impressive one, but the sensor response is not sufficiently high. On the other hand, $\mathrm{Rh}-\mathrm{SnO}_{2} \mathrm{NSh}$ displayed a great response to TEA $\left(\mathrm{R}_{\mathrm{a}} / \mathrm{R}_{\mathrm{g}}=607.2\right.$ for $100 \mathrm{ppm}$ at $325^{\circ} \mathrm{C}$; response/recovery time $\left.=49 \mathrm{~s} / 24 \mathrm{~s}\right)$ with a LOD of $\sim 1 \mathrm{ppm}$, but further investigation is necessary to lower the working temperature. In light of this, Yan et al. reported the hydrothermally synthesized Ag modified $\mathrm{Zn}_{2} \mathrm{SnO}_{4}$ hexagonal nanoflakes-hollow octahedron for the enhanced sensing of TEA [322]. This material performed remarkably in terms of the sensor response $\left(\mathrm{R}_{\mathrm{a}} / \mathrm{R}_{\mathrm{g}}=83.6\right.$ for $50 \mathrm{ppm}$ at $220^{\circ} \mathrm{C}$; response/recovery time $\leq 1 \mathrm{~s} / 24 \mathrm{~s}$ ) with a LOD of $\sim 1 \mathrm{ppm}$. Subsequently, $\mathrm{Zn}_{2} \mathrm{SnO}_{4}$ doped $\mathrm{SnO}_{2}$ hollow NSPs and $\mathrm{CeO}_{2}-\mathrm{SnO}_{2}$ nanoflowers (by Hydrothermal synthesize) were engaged in the sensing investigations of volatile organic amines [323,324]. Wherein, $\mathrm{Zn}_{2} \mathrm{SnO}_{4}$-doped $\mathrm{SnO}_{2}$ hollow NSPs [323] is an exceptional material, which detected the phenylamine $\left(\mathrm{R}_{\mathrm{a}} / \mathrm{R}_{\mathrm{g}}=4.53\right.$ for $50 \mathrm{ppm}$ at $300{ }^{\circ} \mathrm{C}$; response/recovery time $\left.=10 \mathrm{~s} / 4 \mathrm{~s}\right)$ with a LOD of $\sim 1 \mathrm{ppm}$. On the other hand, $\mathrm{CeO}_{2}-\mathrm{SnO}_{2}$ nanoflowers [324] can be noted as an alternative in the TEA sensing at $310^{\circ} \mathrm{C}$.

Similar to diverse nanostructures, materials with porosity were vastly used in the detection of volatile organic amines as described below. $\mathrm{WO}_{3}-\mathrm{SnO}_{2}$ mesoporous nanostructures, $\mathrm{CuO}$ porous particles with diverse morphologies, $\mathrm{In}_{2} \mathrm{O}_{3}$ mesoporous nanocubes, $\mathrm{CeO}_{2}$ porous nanospheres, $\mathrm{Au}$ decahedrons-decorated $\alpha-\mathrm{Fe}_{2} \mathrm{O}_{3}$ porous nanorods, $\mathrm{ZnCo}_{2} \mathrm{O}_{4}$ 
porous nanostructures, $\mathrm{NiCo}_{2} \mathrm{O}_{4}$ porous nanoplates, $\mathrm{SnO}_{2}$ porous thin films, $\mathrm{Fe}_{2} \mathrm{O}_{3} / \mathrm{ZnFe}_{2} \mathrm{O}_{4}$ porous nanocomposite, and $\mathrm{Au}$-Modified $\mathrm{ZnO}$ porous hierarchical nanosheets were reported for the quantification of TMA or TEA as summarized in Table 4 [325-334]. Hydrothermal, solvothermal, calcination, impregnation, template methods, wet-chemical methods, etc, were used to synthesize these porous nanomaterials. Porosity scaled from nano to micro can enhance the capture of volatile amines. A schematic of $\mathrm{WO}_{3}-\mathrm{SnO}_{2}$ mesoporous nanostructure formation and its utilization in sensors is shown in Figure 20 [325].
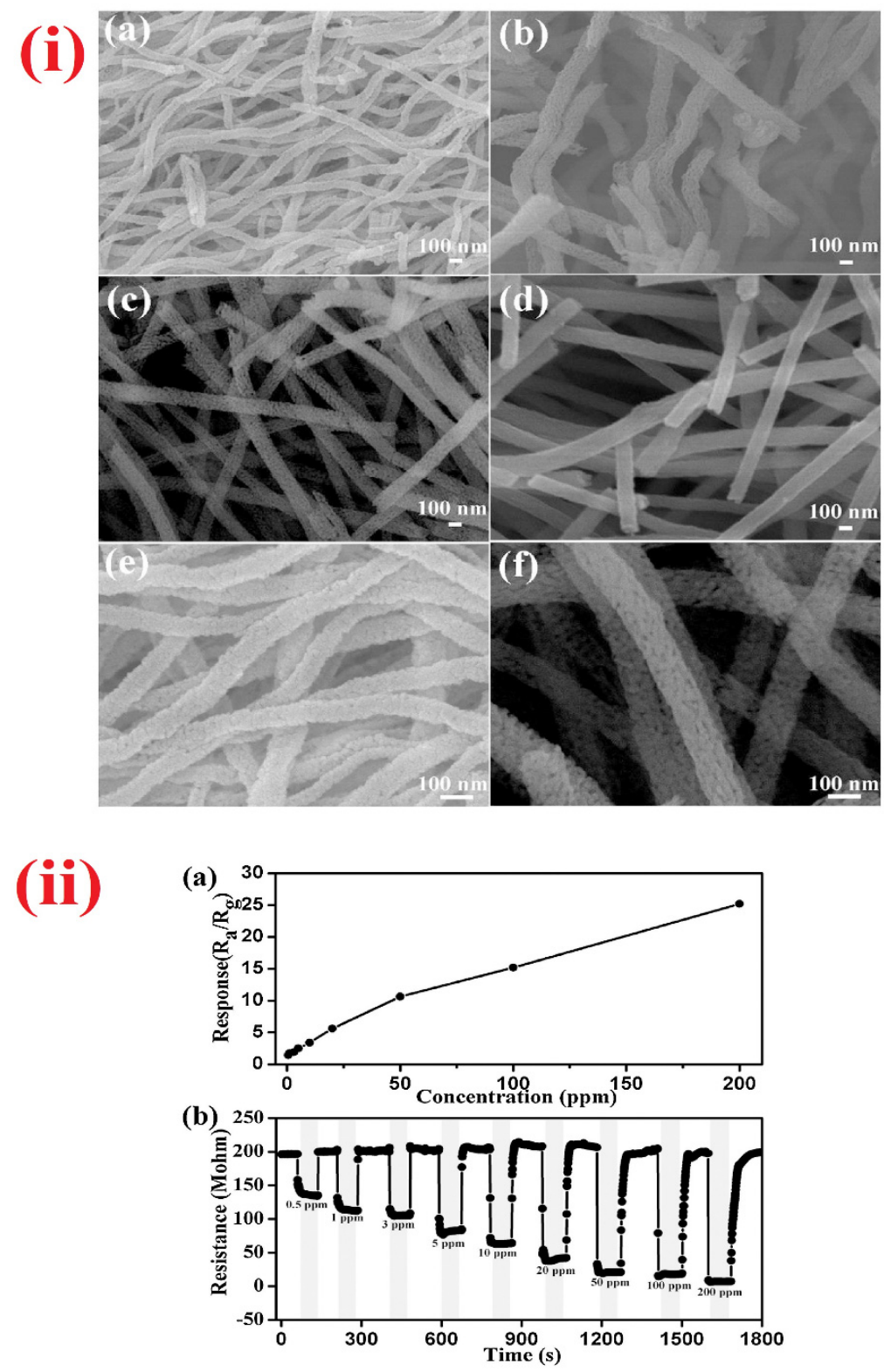

Figure 19. (i) FESEM images of S1 (a), S2 (b), S3 (c), S4 (d), high-magnification SEM images of $\mathrm{S} 1$ (e) and $\mathrm{S} 3$ (f); (Here S1-S4 represents $\mathrm{Al}_{2} \mathrm{O}_{3} / \alpha-\mathrm{Fe}_{2} \mathrm{O}_{3}$ nanofibers); (ii) (a) Gas responses of the $\mathrm{S} 3$ sensor as a function of trimethylamine (TEA) concentrations at $250{ }^{\circ} \mathrm{C}$. (b) Dynamic responserecovery curves of the sensor S3 to different concentrations of TEA at the operating temperature (Reproduced with the permission from Ref. [308]).

Among them, $\mathrm{In}_{2} \mathrm{O}_{3}$ mesoporous nanocubes and Au-Modified $\mathrm{ZnO}$ porous hierarchical nanosheets were engaged in the sensing of TMA with decent LODs [327,334]. Similarly, $\mathrm{CeO}_{2}$ porous nanospheres and $\mathrm{SnO}_{2}$ porous thin films were reported with remarkable performance in sensing of TEA at room temperature [328,332]. In particular, $\mathrm{SnO}_{2}$ porous 
thin films [332] revealed a high sensitivity to TEA $\left(\mathrm{R}_{\mathrm{a}} / \mathrm{R}_{\mathrm{g}}=150.5\right.$ for $10 \mathrm{ppm}$ at RT; response/recovery time $=53 \mathrm{~s} / 120 \mathrm{~s}$ ) with a LOD of $0.11 \mathrm{ppm}$, thereby can be stated as excellent material towards commercialization.

Like other nanostructures, various hierarchical nanostructures were discussed in volatile amines detection. In light of this, $\alpha-\mathrm{Fe}_{2} \mathrm{O}_{3}$ snowflake-like hierarchical nanostructure, $\mathrm{Zn}_{2} \mathrm{SnO}_{4}-\mathrm{ZnO}$ hierarchical nanocomposite, $\mathrm{MoS}_{2} / \mathrm{GO} 3 \mathrm{D}$ hierarchical nanocomposite, $\mathrm{Au}$ NPs decorated $\mathrm{Co}_{3} \mathrm{O}_{4}$ hierarchical nanochains, and $\mathrm{WO}_{3}$ hierarchical flower like spheres were developed and employed in TEA detection [335-339]. These materials were synthesized via the combination of solvothermal, annealing, hydrothermal, calcination, template route, and precipitation methods. These hierarchical nanostructures operate between 205 and $260{ }^{\circ} \mathrm{C}$ to enhance sensor responses to TEA as noted in Table 4. For example, $\mathrm{Au}$ NPs decorated $\mathrm{Co}_{3} \mathrm{O}_{4}$ hierarchical nanochains displayed a sensor response to TEA even at $10 \mathrm{ppm}$ as shown in Figure 21 [338]. However, among the aforementioned hierarchical nanomaterials, $\mathrm{Zn}_{2} \mathrm{SnO}_{4}-\mathrm{ZnO}$ hierarchical nanocomposite [336] revealed a high response to TEA $\left(\mathrm{R}_{\mathrm{a}} / \mathrm{R}_{\mathrm{g}}=175.5\right.$ for $100 \mathrm{ppm}$ at $200{ }^{\circ} \mathrm{C}$; response/recovery time $\left.=12 \mathrm{~s} / 25 \mathrm{~s}\right)$ with a LOD of $0.4 \mathrm{ppm}$, thereby can be noted as an inspiring research in TEA sensors.

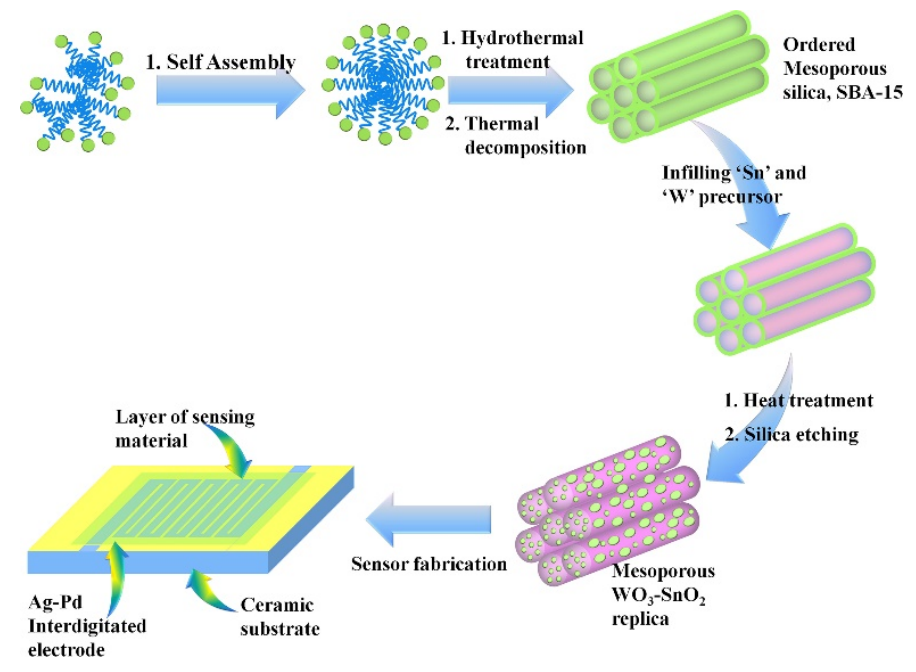

Figure 20. Schematic representation for nanocasting synthesis of mesoporous $\mathrm{WO}_{3}-\mathrm{SnO}_{2}$ by hard templating of SBA-15 (Reproduced with the permission from Ref. [325]).

Apart from the utilization of distinct nano-architectures, numerous diversified nanoshaped materials were employed in the quantitation of volatile organic amines. In view of this, $\mathrm{ZnO} / \mathrm{Au}$ hemishperical nanostructure, $\mathrm{SnO}_{2} / \mathrm{Au} / \mathrm{Fe}_{2} \mathrm{O}_{3}$ nanoboxes, $\mathrm{Au}$ decorated $\mathrm{ZnO}$ nest-like nanostructure, $\mathrm{Pd}$ doped $\mathrm{ZnO}$ agaric like nanostructure, $\mathrm{Co}_{3} \mathrm{O}_{4} @ \mathrm{MnO}_{2}$ shishkebab like nanostructure, and $\mathrm{Au} @ \mathrm{ZnO}$ core-shell nanostructure were demonstrated in TEA and aniline detection as noted in Table 4 [340-345]. In particular, Pd-doped $\mathrm{ZnO}$ agaric like nanostructure [343] was used in aniline sensing with a high response $\left(R_{a} / R_{g}=182\right.$ for $100 \mathrm{ppm}$ at $280{ }^{\circ} \mathrm{C}$; response/recovery time $=29 \mathrm{~s} / 23 \mathrm{~s}$ ) with a LOD of $0.5 \mathrm{ppm}$, thereby is noted as a distinct research. Among the other materials in TEA discrimination, $\mathrm{Au} @ \mathrm{ZnO}$ core-shell nanostructure [345] is an exceptional material with excellent sensor performance at $50{ }^{\circ} \mathrm{C}\left(\mathrm{R}_{\mathrm{a}} / \mathrm{R}_{\mathrm{g}}=12.2 \%\right.$ for $5 \mathrm{ppm}$; response/recovery time is $\left.27 \mathrm{~s} / 46 \mathrm{~s}\right)$ and a LOD of $\sim 1 \mathrm{ppm}$. In parallel to diverse nanostructures, composite materials without any structural modifications were also utilized in TEA and TMA sensors. Materials such as $\mathrm{ZnO} / \mathrm{ZnFe}_{2} \mathrm{O}_{4}$ composites, $\mathrm{Au} / \mathrm{Co}_{3} \mathrm{O}_{4} / \mathrm{W}_{18} \mathrm{O}_{49}$ hollow composite nanospheres, $\alpha$ $\mathrm{Fe}_{2} \mathrm{O}_{3} @ \alpha-\mathrm{MoO}_{3}$ composite, $\mathrm{CuO} / \mathrm{ZnO} 3 \mathrm{D}$ diamond shaped MOF, rGO decorated Wdoped $\mathrm{BiVO}_{4}$ hierarchical nanocomposite, and $\mathrm{Au} @ \mathrm{MoS}_{2}$ nanocomposite were effectively consumed in TEA or TMA detection [346-351]. Herein, $\mathrm{ZnO} / \mathrm{ZnFe}_{2} \mathrm{O}_{4}$ composite displayed a good sensitivity to TEA under irradiation $\left(\mathrm{R}_{\mathrm{a}} / \mathrm{R}_{\mathrm{g}} \geq 9\right.$ for $500 \mathrm{ppm}$ at $\left.80^{\circ} \mathrm{C}\right)$ and requires additional interrogations to enhance the response [346]. Similarly, p-n $\mathrm{CuO} / \mathrm{ZnO}$ 3D 
diamond shaped MOF composite authenticated the selectivity to TEA and methanol at $220^{\circ} \mathrm{C}$ and $260{ }^{\circ} \mathrm{C}$, respectively [349]. However, this composite showed a better response to TEA $\left(R_{a} / R_{g} \geq 400\right.$ for $500 \mathrm{ppm}$; response/recovery time $\left.=11 \mathrm{~s} / \sim 60 \mathrm{~min}\right)$ than that of methanol with a LOD of $0.175 \mathrm{ppm}$. However, this research still needs further work. Compared to the other composite materials, $\mathrm{Au} @ \mathrm{MoS}_{2}$ nanocomposite is a unique material with its sensory response to TEA at $30^{\circ} \mathrm{C}\left(\mathrm{R}_{\mathrm{a}} / \mathrm{R}_{\mathrm{g}}=44\right.$ for $50 \mathrm{ppm}$; response/recovery time $=9 \mathrm{~s} / 91 \mathrm{~s}$ ) and a LOD of $\sim 2 \mathrm{ppm}$ as noted in Table 4 [351].

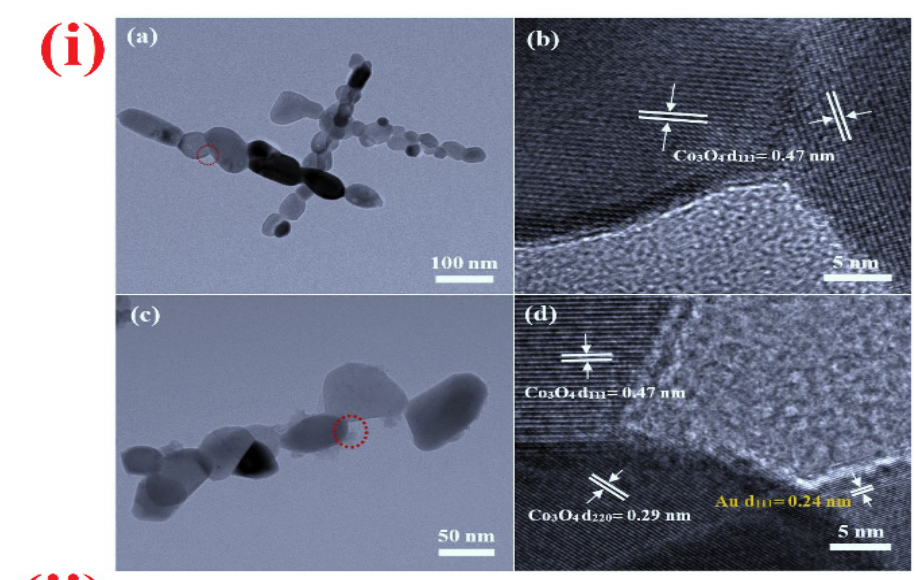

(ii)
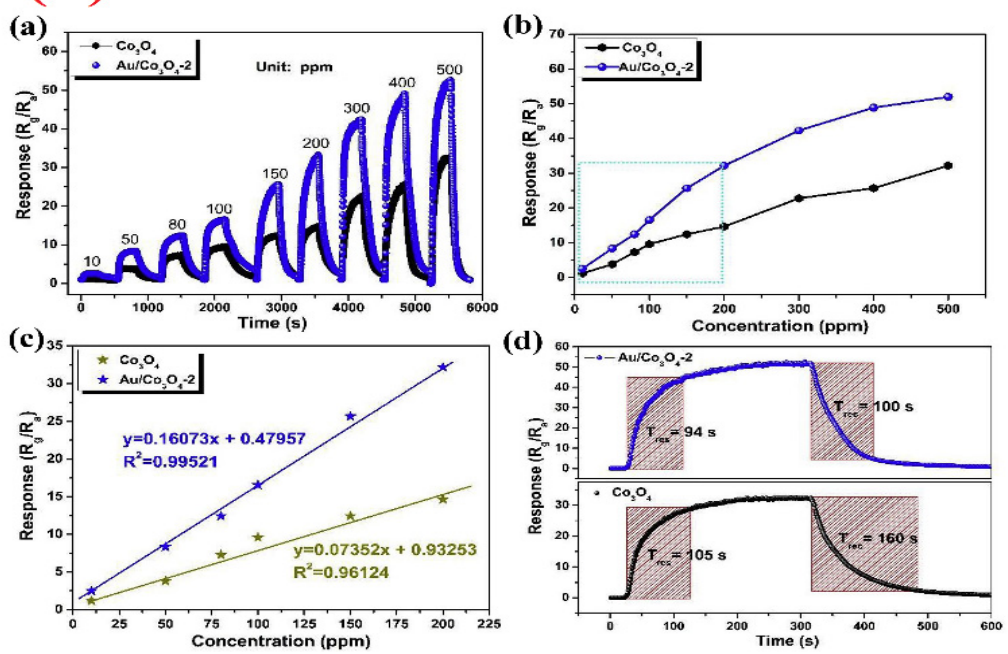

Figure 21. (i) TEM and HRTEM images of the prepared $(\mathbf{a}, \mathbf{b})$ pure and $(\mathbf{c}, \mathbf{d}) \mathrm{Au} / \mathrm{Co}_{3} \mathrm{O}_{4}$ nanochains $\left(\mathrm{Au} / \mathrm{Co}_{3} \mathrm{O}_{4}-2\right)$ (ii) (a) Dynamic response-recover curves of the nanochain sensors towards various concentrations of TEA and their concentration-dependent responses within the TEA concentration range of (b) $10 \mathrm{e} 500 \mathrm{ppm}$ and (c)10-200 ppm; (d) the response and recovery curves of the sensors towards 300 ppm TEA (Reproduced with the permission from Ref. [338]).

Table 4. Detection concentration, response/recovery time, operating temperature (Temp.) and LODs to volatile organic amine gas by diverse nanostructured materials.

\begin{tabular}{ccccccc}
\hline Materials/Nanostructure & Analyte/Concentration & $\begin{array}{c}\text { Gas Response } \\
\left(\mathbf{R}_{\text {air }} / \mathbf{R}_{\text {gas }}\right)\end{array}$ & Response/Recovery & Temp. & LOD & Ref \\
\hline $\begin{array}{c}\mathrm{Co}_{3} \mathrm{O}_{4} / \mathrm{ZnO} / \mathrm{hybrid} \\
\text { nanoparticles }\end{array}$ & Triethylamine/200 ppm & 282.3 & $25 \mathrm{~s} / 36 \mathrm{~s}$ & $280{ }^{\circ} \mathrm{C}$ & $\sim 10 \mathrm{ppm}$ & {$[296]$} \\
\hline $\begin{array}{c}\text { Ho-doped } \\
\mathrm{SnO}_{2} / \text { nanoparticles }\end{array}$ & Triethylamine $/ 50 \mathrm{ppm}$ & 12 & $2 \mathrm{~s} / 2 \mathrm{~min}$ & $175{ }^{\circ} \mathrm{C}$ & $\sim 5 \mathrm{ppm}$ & {$[297]$} \\
\hline $\mathrm{CuCrO} /$ nanoparticles & Triethylamine $/ 100 \mathrm{ppm}$ & $\sim 5$ & $90 \mathrm{~s} / 120 \mathrm{~s}$ & $140{ }^{\circ} \mathrm{C}$ & $\sim 10 \mathrm{ppm}$ & {$[298]$} \\
\hline $\mathrm{Ag} / \mathrm{Pt} / \mathrm{W}_{18} \mathrm{O}_{49} /$ nanowires & Triethylamine $/ 50 \mathrm{ppm}$ & 813 & $15 \mathrm{~s} / 35 \mathrm{~s}($ for $2 \mathrm{ppm})$ & $240^{\circ} \mathrm{C}$ & $0.071 \mathrm{ppm}$ & {$[299]$} \\
\hline
\end{tabular}


Table 4. Cont

\begin{tabular}{|c|c|c|c|c|c|c|}
\hline Materials/Nanostructure & Analyte/Concentration & $\begin{array}{l}\text { Gas Response } \\
\left(\mathbf{R}_{\text {air }} / \mathbf{R}_{\text {gas }}\right)\end{array}$ & Response/Recovery & Temp. & LOD & Ref \\
\hline $\begin{array}{c}1 \mathrm{D} \mathrm{SnO} \mathrm{S}_{2} \text { coated } \mathrm{ZnO} / \text { hybrid } \\
\text { nanowires }\end{array}$ & n-Butylamine/10 ppm & 7.4 & $40 \mathrm{~s} / 80 \mathrm{~s}$ & $240^{\circ} \mathrm{C}$ & $\sim 1 \mathrm{ppm}$ & [300] \\
\hline $\begin{array}{c}\mathrm{V}_{2} \mathrm{O}_{5} \text {-decorated } \\
\alpha-\mathrm{Fe}_{2} \mathrm{O}_{3} / \text { nanorods }\end{array}$ & Diethylamine/100 ppm & 8.9 & $2 \mathrm{~s} / 40 \mathrm{~s}$ & $350^{\circ} \mathrm{C}$ & $\sim 5 \mathrm{ppm}$ & [301] \\
\hline $\begin{array}{l}\text { Au NPs decorated } \\
\mathrm{WO}_{3} / \text { nanorods }\end{array}$ & Trimethyl-amine/100 ppm & 76.7 & $6 \mathrm{~s} / 7 \mathrm{~s}$ & $280^{\circ} \mathrm{C}$ & $\sim 5 \mathrm{ppm}$ & [302] \\
\hline $\begin{array}{l}\mathrm{Ag} \mathrm{NPs} \text { decorated } \\
\alpha-\mathrm{MoO}_{3} / \text { nanorods }\end{array}$ & Triethylamine/100 ppm & 408.6 & $3 \mathrm{~s} / 107 \mathrm{~s}$ & $200^{\circ} \mathrm{C}$ & $0.035 \mathrm{ppm}$ & [303] \\
\hline $\mathrm{Cr}$ doped $\alpha-\mathrm{MoO}_{3} /$ nanorods & Triethylamine/100 ppm & 150.25 & $7 \mathrm{~s} / 80 \mathrm{~s}$ & $200^{\circ} \mathrm{C}$ & $\sim 1 \mathrm{ppm}$ & [304] \\
\hline Acidic $\alpha-\mathrm{MoO}_{3} /$ nanorods & Triethylamine/100 ppm & 101.74 & $4 \mathrm{~s} / 88 \mathrm{~s}$ & $300^{\circ} \mathrm{C}$ & $0.1 \mathrm{ppm}$ & [305] \\
\hline $\begin{array}{c}\mathrm{Au} @ \mathrm{SnO}_{2} / \alpha-\mathrm{Fe}_{2} \mathrm{O}_{3} / \text { core- } \\
\text { shell nanoneedles on alumina } \\
\text { tubes }\end{array}$ & Triethylamine/100 ppm & 39 & $4 \mathrm{~s} / 203 \mathrm{~s}$ & $300^{\circ} \mathrm{C}$ & $\sim 2 \mathrm{ppm}$ & [307] \\
\hline $\mathrm{Al}_{2} \mathrm{O}_{3} / \alpha-\mathrm{Fe}_{2} \mathrm{O}_{3} /$ nanofibers & Triethylamine/100 ppm & 15.19 & $1 \mathrm{~s} / 17 \mathrm{~s}$ & $250^{\circ} \mathrm{C}$ & $\sim 0.5 \mathrm{ppm}$ & [308] \\
\hline $\begin{array}{l}\mathrm{In}_{2} \mathrm{O}_{3} / \text { hierarchical nanofibers } \\
\text { (with nanoparticles) }\end{array}$ & Triethylamine/50 ppm & 87.8 & $148 \mathrm{~s} / 40 \mathrm{~min}$ & $40^{\circ} \mathrm{C}$ & $\sim 5 \mathrm{ppm}$ & [309] \\
\hline $\mathrm{Nb}$ doped $\mathrm{TiO}_{2} /$ nanotubes & Dimethyl-amine/50 ppm & 9.1 & $\geq 300 \mathrm{~s}$ (for both) & $300^{\circ} \mathrm{C}$ & $\sim 5 \mathrm{ppm}$ & [312] \\
\hline $\begin{array}{l}\mathrm{Au} \mathrm{NPs} \text { decorated } \\
\mathrm{MoO}_{3} / \text { nanobelts }\end{array}$ & Trimethyl-amine/50 ppm & 70 & $6 s / 9 s$ & $280^{\circ} \mathrm{C}$ & $\sim 5 \mathrm{ppm}$ & [313] \\
\hline $\mathrm{W}$ doped $\mathrm{MoO}_{3} /$ nanobelts & Trimethyl-amine $/ 50 \mathrm{ppm}$ & 13.8 & $6 \mathrm{~s} / 11 \mathrm{~s}$ & $280^{\circ} \mathrm{C}$ & $\sim 5 \mathrm{ppm}$ & [314] \\
\hline $\begin{array}{c}\mathrm{RuO}_{2} \mathrm{NPs}_{\text {decorated }} \\
\mathrm{MoO}_{3} / \text { nanobelts }\end{array}$ & Triethylamine/10 ppm & 75 & $2 \mathrm{~s} / 10 \mathrm{~s}$ & $260^{\circ} \mathrm{C}$ & $\sim 1 \mathrm{ppm}$ & [315] \\
\hline $\mathrm{ZnO}-\mathrm{SnO}_{2} /$ nanobelts & Triethylamine/100 ppm & 9.9 & $1.8 \mathrm{~s} / 18 \mathrm{~s}$ & $220^{\circ} \mathrm{C}$ & $\sim 1 \mathrm{ppm}$ & [316] \\
\hline $\mathrm{In}_{2} \mathrm{O}_{3} /$ nanocubes & Triethylamine/100 ppm & 175 & $11 \mathrm{~s} / 14 \mathrm{~s}$ & $180^{\circ} \mathrm{C}$ & $\sim 10 \mathrm{ppm}$ & [317] \\
\hline $\mathrm{WO}_{3} /$ nanosheets & Triethylamine/1000 ppm & $\sim 14$ & NA & $\begin{array}{l}\text { Room } \\
\text { Temp. }\end{array}$ & $\sim 5 \mathrm{ppm}$ & [318] \\
\hline $\mathrm{Au} @ \mathrm{ZnO}-\mathrm{SnO}_{2} /$ nanosheets & Triethylamine/100 ppm & 115 & $7 \mathrm{~s} / 30 \mathrm{~s}$ & $300^{\circ} \mathrm{C}$ & $\sim 2 \mathrm{ppm}$ & [319] \\
\hline $\begin{array}{l}\mathrm{TiO}_{2} \mathrm{NPs} \text { decorated } \\
\mathrm{CuO} / \text { nanosheets }\end{array}$ & Triethylamine/5 ppm & 12.7 & $45 \mathrm{~s} / 202 \mathrm{~s}$ & $160^{\circ} \mathrm{C}$ & $0.5 \mathrm{ppm}$ & [320] \\
\hline $\mathrm{Rh}-\mathrm{SnO}_{2} /$ nanosheets & Triethylamine/100 ppm & 607.2 & $49 \mathrm{~s} / 24 \mathrm{~s}$ & $325^{\circ} \mathrm{C}$ & $\sim 1 \mathrm{ppm}$ & [321] \\
\hline $\begin{array}{c}\mathrm{Ag} \text { modified } \\
\mathrm{Zn}_{2} \mathrm{SnO}_{4} / \text { hexagonal } \\
\text { nanoflakes- hollow } \\
\text { octahedron }\end{array}$ & Triethylamine/50 ppm & 83.6 & $<1 \mathrm{~s} / 20 \mathrm{~s}$ & $220^{\circ} \mathrm{C}$ & $\sim 1 \mathrm{ppm}$ & [322] \\
\hline $\begin{array}{c}\mathrm{Zn}_{2} \mathrm{SnO}_{4} \text { - doped } \\
\mathrm{SnO}_{2} / \text { hollow nanospheres }\end{array}$ & Phenylamine/50 ppm & 4.53 & $10 \mathrm{~s} / 12 \mathrm{~s}$ & $300^{\circ} \mathrm{C}$ & $\sim 1 \mathrm{ppm}$ & [323] \\
\hline $\mathrm{CeO}_{2}-\mathrm{SnO}_{2} /$ nanoflowers & Triethylamine/200 ppm & 252.2 & NA & $310^{\circ} \mathrm{C}$ & $\sim 20 \mathrm{ppm}$ & [324] \\
\hline $\begin{array}{c}\mathrm{WO}_{3}-\mathrm{SnO}_{2} / \text { mesoporous } \\
\text { nanostructure }\end{array}$ & Triethylamine/50 ppm & 87 & $6 \mathrm{~s} / 7 \mathrm{~s}$ & $220^{\circ} \mathrm{C}$ & $\sim 1 \mathrm{ppm}$ & [325] \\
\hline $\begin{array}{l}\mathrm{CuO} / \text { porous particles with } \\
\text { diverse morphologies }\end{array}$ & Triethylamine/100 ppm & $5.6-102$ & $<40 \mathrm{~s} /<260 \mathrm{~s}$ & $230^{\circ} \mathrm{C}$ & $\sim 5 \mathrm{ppm}$ & [326] \\
\hline $\begin{array}{c}\mathrm{In}_{2} \mathrm{O}_{3} / \text { mesoporous } \\
\text { nanocubes }\end{array}$ & Trimethyl-amine/10 ppm & 57 & $4 \mathrm{~s} / 11 \mathrm{~s}$ & $160^{\circ} \mathrm{C}$ & $\sim 5 \mathrm{ppm}$ & [327] \\
\hline $\mathrm{CeO}_{2} /$ porous nanospheres & Triethylamine/100 ppm & 4.67 & $13 \mathrm{~s} /<230 \mathrm{~s}$ & $\begin{array}{l}\text { Room } \\
\text { Temp. }\end{array}$ & $\sim 5 \mathrm{ppm}$ & [328] \\
\hline $\begin{array}{l}\text { Au decahedrons-decorated } \\
\alpha-\mathrm{Fe}_{2} \mathrm{O}_{3} / \text { porous nanorods }\end{array}$ & Triethylamine/50 ppm & 17 & $12 \mathrm{~s} / 18 \mathrm{~s}$ & $40^{\circ} \mathrm{C}$ & $1 \mathrm{ppm}$ & [329] \\
\hline $\begin{array}{l}\mathrm{ZnCo}_{2} \mathrm{O}_{4} / \text { porous nano- } \\
\text { structures }\end{array}$ & Triethylamine/100 ppm & 14 & $7 \mathrm{~s} / 57 \mathrm{~s}$ & $200^{\circ} \mathrm{C}$ & $\sim 5 \mathrm{ppm}$ & [330] \\
\hline $\mathrm{NiCO}_{2} \mathrm{O}_{4} /$ porous nanoplates & Triethylamine/10 ppm & 2.58 & $<33 \mathrm{~s} / 42 \mathrm{~s}$ & $220^{\circ} \mathrm{C}$ & $0.5 \mathrm{ppm}$ & [331] \\
\hline $\mathrm{SnO}_{2} /$ porous thin films & Triethylamine/10 ppm & 150.5 & $53 \mathrm{~s} / 120 \mathrm{~s}$ & $\begin{array}{l}\text { Room } \\
\text { Temp. }\end{array}$ & $0.11 \mathrm{ppm}$ & [332] \\
\hline
\end{tabular}


Table 4. Cont.

\begin{tabular}{|c|c|c|c|c|c|c|}
\hline Materials/Nanostructure & Analyte/Concentration & $\begin{array}{l}\text { Gas Response } \\
\left(\mathbf{R}_{\text {air }} / \mathbf{R}_{\text {gas }}\right)\end{array}$ & Response/Recovery & Temp. & LOD & Ref \\
\hline $\begin{array}{c}\mathrm{Fe}_{2} \mathrm{O}_{3} / \mathrm{ZnFe}_{2} \mathrm{O}_{4} / \text { porous } \\
\text { nano- composite }\end{array}$ & Triethylamine/20 ppm & 60.24 & $2 \mathrm{~s} / 7 \mathrm{~s}$ & $300^{\circ} \mathrm{C}$ & $0.2 \mathrm{ppm}$ & [333] \\
\hline $\begin{array}{l}\text { Au-Modified } \mathrm{ZnO} / \text { porous } \\
\text { hierarchical nanosheets }\end{array}$ & Trimethyl-amine/30 ppm & 65.8 & $3.3 \mathrm{~s} / 64 \mathrm{~s}$ & $260^{\circ} \mathrm{C}$ & $0.01 \mathrm{ppm}$ & [334] \\
\hline $\begin{array}{c}\alpha-\mathrm{Fe}_{2} \mathrm{O}_{3} / \text { snowflake-like } \\
\text { hierarchical nanostructure }\end{array}$ & Trimethyl-amine/100 ppm & 10.9 & $0.9 \mathrm{~s} / 1.5 \mathrm{~s}$ & $260^{\circ} \mathrm{C}$ & $\sim 5 \mathrm{ppm}$ & [335] \\
\hline $\begin{array}{c}\mathrm{Zn}_{2} \mathrm{SnO}_{4}-\mathrm{ZnO} / \text { hierarchical } \\
\text { nano- composite }\end{array}$ & Triethylamine/100 ppm & 175.5 & $12 \mathrm{~s} / 25 \mathrm{~s}$ & $200^{\circ} \mathrm{C}$ & $0.4 \mathrm{ppm}$ & [336] \\
\hline $\begin{array}{c}\mathrm{MoS}_{2} / \mathrm{GO} / 3 \mathrm{D} \text { hierarchical } \\
\text { nano- composite }\end{array}$ & Triethylamine/100 ppm & 192 & $20 \mathrm{~s} / 18 \mathrm{~s}$ & $260^{\circ} \mathrm{C}$ & $1 \mathrm{ppm}$ & [337] \\
\hline $\begin{array}{c}\mathrm{Au} \mathrm{NPs} \mathrm{decorated} \\
\mathrm{Co}_{3} \mathrm{O}_{4} / \text { hierarchical } \\
\text { nanochains }\end{array}$ & Triethylamine/300 ppm & $>40$ & $94 \mathrm{~s} / 100 \mathrm{~s}$ & $210^{\circ} \mathrm{C}$ & $\sim 10$ ppm & [338] \\
\hline $\begin{array}{l}\mathrm{WO}_{3} / \text { hierarchical flower like } \\
\text { spheres }\end{array}$ & Triethylamine/10 ppm & 11.6 & $3 \mathrm{~s} / 55 \mathrm{~s}$ & $205^{\circ} \mathrm{C}$ & $0.083 \mathrm{ppm}$ & [339] \\
\hline $\begin{array}{c}\mathrm{ZnO} / \mathrm{Au} / \text { hemishperical } \\
\text { nanostructure }\end{array}$ & Triethylamine/100 ppm & 104.8 & $5 \mathrm{~s} / 2 \mathrm{~s}$ & $260^{\circ} \mathrm{C}$ & $\sim 10 \mathrm{ppm}$ & [340] \\
\hline $\mathrm{SnO}_{2} / \mathrm{Au} / \mathrm{Fe}_{2} \mathrm{O}_{3} /$ nanoboxes & Triethylamine/100 ppm & 126.84 & $7 \mathrm{~s} / 10 \mathrm{~s}$ & $240^{\circ} \mathrm{C}$ & $0.05 \mathrm{ppm}$ & [341] \\
\hline $\begin{array}{c}\mathrm{Au} \text { decorated } \mathrm{ZnO} / \text { nest-like } \\
\text { nanostructure }\end{array}$ & Triethylamine/200 ppm & 625 & $4 \mathrm{~s} / 26 \mathrm{~s}$ & $320^{\circ} \mathrm{C}$ & $1 \mathrm{ppm}$ & [342] \\
\hline $\begin{array}{c}\mathrm{Pd} \text { doped } \mathrm{ZnO} / \text { agaric like } \\
\text { nanostructure }\end{array}$ & Aniline/100 ppm & 182 & $29 \mathrm{~s} / 23 \mathrm{~s}$ & $280^{\circ} \mathrm{C}$ & $0.5 \mathrm{ppm}$ & [343] \\
\hline $\begin{array}{c}\mathrm{Co}_{3} \mathrm{O}_{4} @ \mathrm{MnO}_{2} / \text { shish-kebab } \\
\text { like nanostructure }\end{array}$ & Triethylamine/100 ppm & 9.13 & $93 \mathrm{~s} / 92 \mathrm{~s}$ & $250^{\circ} \mathrm{C}$ & $\sim 10 \mathrm{ppm}$ & [344] \\
\hline $\begin{array}{c}\mathrm{Au} @ \mathrm{ZnO} / \text { core-shell } \\
\text { nanostructure }\end{array}$ & Triethylamine/5 ppm & $12.2 \%$ & $27 \mathrm{~s} / 46 \mathrm{~s}$ & $50^{\circ} \mathrm{C}$ & $\sim 1 \mathrm{ppm}$ & [345] \\
\hline $\begin{array}{c}\mathrm{Au} / \mathrm{Co}_{3} \mathrm{O}_{4} / \mathrm{W}_{18} \mathrm{O}_{49} / \text { hollow } \\
\text { composite nanospheres }\end{array}$ & Triethylamine/2 ppm & 16.7 & $9 \mathrm{~s} / 14 \mathrm{~s}$ & $270^{\circ} \mathrm{C}$ & $0.081 \mathrm{ppm}$ & [347] \\
\hline $\begin{array}{l}\alpha-\mathrm{Fe}_{2} \mathrm{O}_{3} @ \alpha-\mathrm{MoO}_{3} / \text { nano- } \\
\text { composite }\end{array}$ & Triethylamine/50 ppm & 76 & $4 \mathrm{~s} / 370 \mathrm{~s}$ & $280^{\circ} \mathrm{C}$ & $\sim 2 \mathrm{ppm}$ & [348] \\
\hline $\begin{array}{l}\text { rGO decorated W doped } \\
\mathrm{BiVO}_{4} / \text { hierarchical nano- } \\
\text { composite }\end{array}$ & Trimethyl-amine/20 ppm & 12.8 & $16 \mathrm{~s} / \mathrm{NA}$ & $135^{\circ} \mathrm{C}$ & $0.63 \mathrm{ppm}$ & [350] \\
\hline $\mathrm{Au} @ \mathrm{MoS}_{2} /$ nano- composite & Triethylamine/50 ppm & 44 & $9 \mathrm{~s} / 91 \mathrm{~s}$ & $30^{\circ} \mathrm{C}$ & $\sim 2 \mathrm{ppm}$ & [351] \\
\hline
\end{tabular}

$\mathrm{NA}=$ Not available; Temp. $=$ Temperature $\mathrm{s}=$ seconds $; \mathrm{min}=$ minutes .

\section{Volatile Hydrocarbons Detection by Distinct Nanostructures}

Following the similar mechanism mentioned earlier in acetone and alcohols discrimination, hydrocarbons sensors were reported using various nanostructured materials. Nanoparticles were oftenly investigated by many researchers. Chen et al. reported Ag$\mathrm{LaFeO}_{3} \mathrm{NPs}$ developed via two different tactics like MIP and lotus-leaf templated synthesis and engaged in xylene detection at $99^{\circ} \mathrm{C}$ and $125^{\circ} \mathrm{C}$, correspondingly [352,353]. As noted in Table 5, $\mathrm{Ag}-\mathrm{LaFeO}_{3} \mathrm{NPs}$ synthesized via MIP tactic seems to be good in terms of sensor response $\left(\mathrm{R}_{\mathrm{a}} / \mathrm{R}_{\mathrm{g}}=36.2\right.$ for $5 \mathrm{ppm}$ at $99^{\circ} \mathrm{C}$; response/recovery time $\left.=114 \mathrm{~s} / 55 \mathrm{~s}\right)$ with a LOD of $<1 \mathrm{ppm}$, thereby $\mathrm{Ag}-\mathrm{LaFeO}_{3} \mathrm{NPs}$ can be attested as an inspiring candidate in xylene sensors. Similar to above studies, Au loaded $\mathrm{ZnO} N$ Ps and cobalt porphyrin (CoPP)functionalized $\mathrm{TiO}_{2} \mathrm{NPs}$ were described for the quantitation of xylene and BTX (Benzene, Toluene and Xylene) vapors at $377^{\circ} \mathrm{C}$ and $240{ }^{\circ} \mathrm{C}$, respectively $[354,355]$. Wherein, cobalt porphyrin (CoPP)-functionalized $\mathrm{TiO}_{2} \mathrm{NPs}$ showed a high response $\left(\mathrm{R}_{\mathrm{a}} / \mathrm{R}_{\mathrm{g}} \geq 5\right.$ for $10 \mathrm{ppm}$ at $240{ }^{\circ} \mathrm{C}$; response/recovery time $=40 \mathrm{~s} / 80 \mathrm{~s}$ ) with a LOD of $0.005 \mathrm{ppm}$ [355]. However, further studies are needed to minimize the operating temperatures in both cases. Other than NPs, Park and co-workers described the utility of In-doped ZnO Quantum dots (QDs) towards the sensing of acetylene gas at $400{ }^{\circ} \mathrm{C}$ with the LOD down to sub-ppm level 
(0.1 ppm) [356]. However, the working temperature in this report was high, which requires more work. In view of this, $\mathrm{Xu}$ et al. exploited the BTEX (Benzene, Toluene, Ethyl benzene, and Xylene) detection by MOF derived nanocrystals at room temperature [357]. However, anti-interference studies with this material is still in question, thereby cannot be attested as excellent work.

To discriminate the volatile toluene, $\alpha-\mathrm{Fe}_{2} \mathrm{O}_{3} / \mathrm{SnO}_{2} \mathrm{NW}$ arrays and Pt NPs sensitized Si NW-TeO $\mathrm{NW}_{2}$ NWted from ultrasonic spray pyrolysis, hydrothermal, and sputtering tactics) were proposed with sensor responses $>40$ at $90{ }^{\circ} \mathrm{C}$ and $200{ }^{\circ} \mathrm{C}$, respectively, as noted in Table 4 [358,359]. Such NWs-based sensory research is noted as an innovative one. Pd NPs decorated $\mathrm{TiO}_{2}$ NRds were employed in the sensing of liquefied petroleum gas (LPG), but further interrogations are required for authentication [360]. Following above work, Lee et al. fabricated the cobalt porphyrin (CoPP)-ZNO NRds towards the detection of Toluene $\left(\mathrm{R}_{\mathrm{a}} / \mathrm{R}_{\mathrm{g}}=3.3\right.$ for $\left.10 \mathrm{ppm}\right)$ and estimated a LOD of $0.002 \mathrm{ppm}$ [361]. Moreover, this material can operates in the 0 to $85 \%$ humid conditions, thereby attest as a nice work. Qin and co-workers described the Y-doped or undoped $\alpha-\mathrm{MoO}_{3}$ nanoarrays towards the enhanced sensing of Xylene at $370{ }^{\circ} \mathrm{C}$ as noted in Table 5 [362,363]. As shown in Figure 22, authors conformed the $1 \% \mathrm{Y}$ doping over $\alpha-\mathrm{MoO}_{3}$ nanoarrays displayed an improved sensor response than that of other doping concentrations or non-doping ( $0,3 \%$, and $5 \%$ ). However, both works do not merit the commercialization due to the higher operating temperature.

(a)
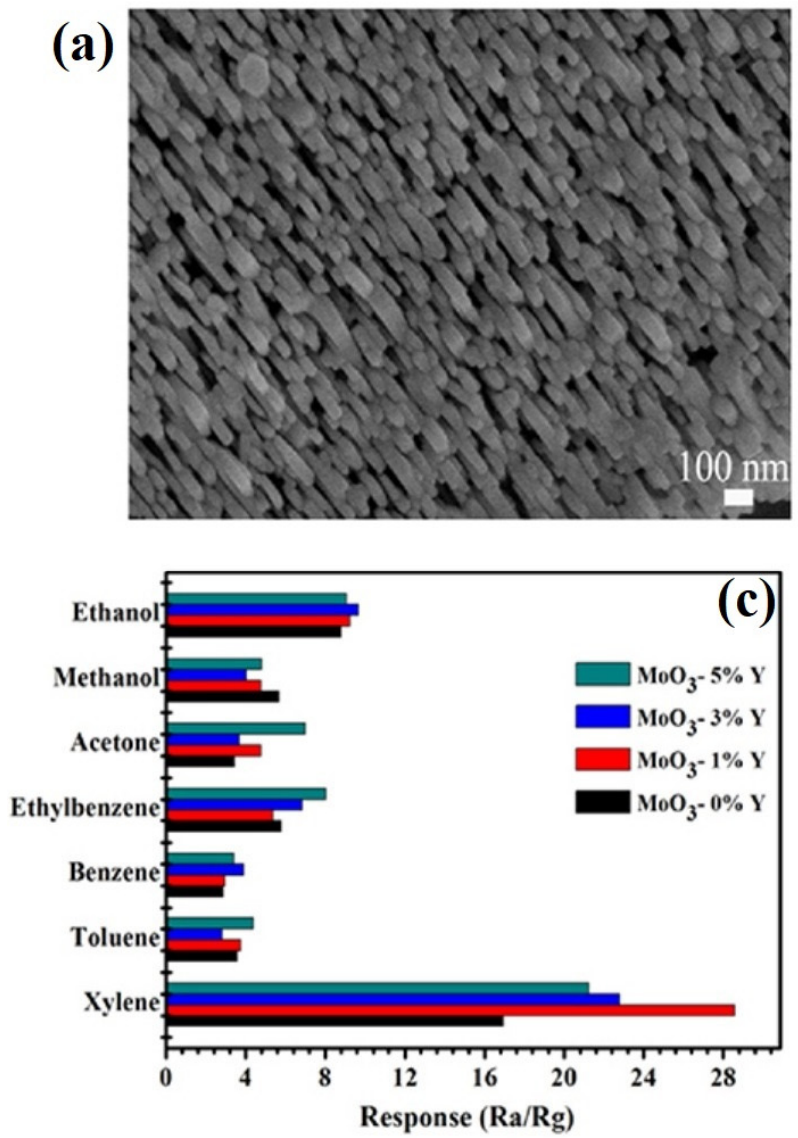

(b)
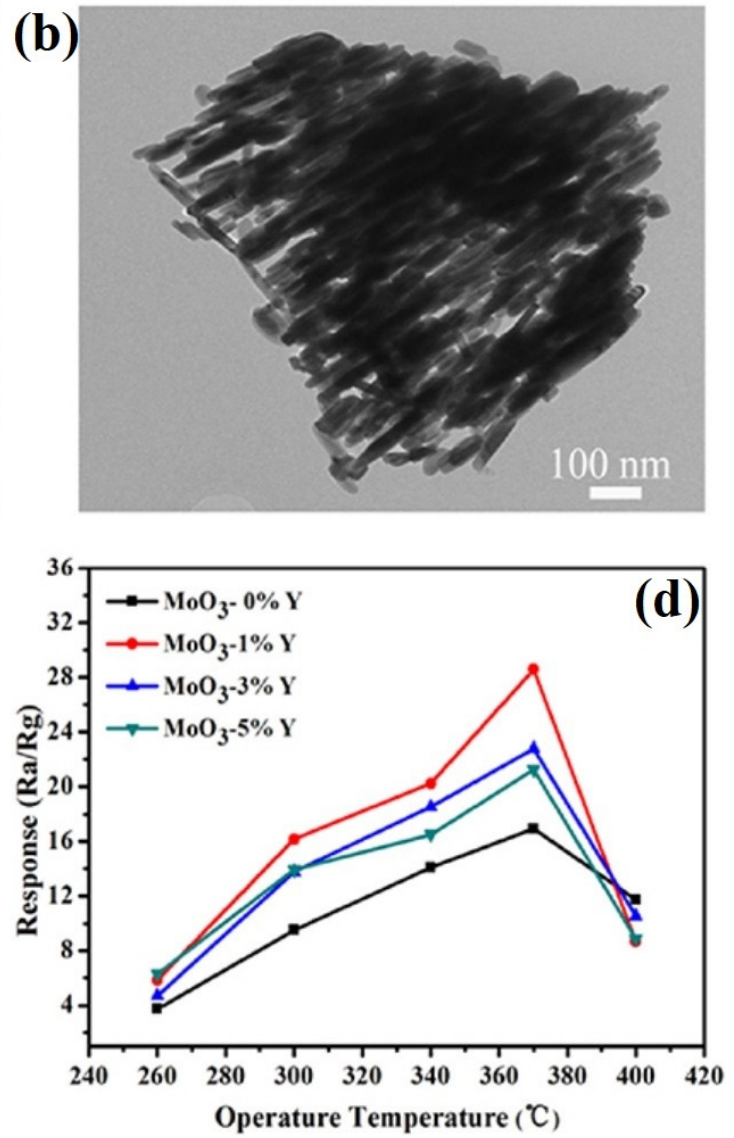

Figure 22. (a,b) The SEM and TEM images of nanoarrays; (c) Response curve of samples to 100 ppm different Gases at the working temperature of $370{ }^{\circ} \mathrm{C}$; (d) Response curve of samples to $100 \mathrm{ppm}$ xylene at the different working temperature (Reproduced with the permission from Ref. [363]).

Nanofibers, such as MOF-driven metal-embedded metal oxide ( $\left.\mathrm{Pd} @ \mathrm{ZnO}-\mathrm{WO}_{3}\right)$ nanofibers, $\mathrm{V}_{2} \mathrm{O}_{5}$ nanofibers, and $\mathrm{Pd}$ functionalized $\mathrm{SnO}_{2}$ nanofibers were engaged in the determination of toluene, xylene, and butane, correspondingly [364-366]. Pd@ZnO- $\mathrm{WO}_{3}$ nanofibers 
showed a high response to toluene $\left(\mathrm{R}_{\mathrm{a}} / \mathrm{R}_{\mathrm{g}}=22.22\right.$ for $1 \mathrm{ppm}$ at $350{ }^{\circ} \mathrm{C}$; response/recovery time $\leq 20 \mathrm{~s}$ /not available) with a LOD of $0.1 \mathrm{ppm}$, thereby become an impressive candidate apart from the working temperature [356]. Subsequently, $\mathrm{V}_{2} \mathrm{O}_{5}$ nanofibers were noted as highly remarkable material to be employed in xylene detection at room temperature $\left(\mathrm{R}_{\mathrm{a}} / \mathrm{R}_{\mathrm{g}}=191\right.$ for $500 \mathrm{ppm}$ at RT; response/recovery time $=80 \mathrm{~s} / 50 \mathrm{~s}$ (for $\left.100 \mathrm{ppm}\right)$ ) with an estimated LOD of $\sim 5 \mathrm{ppm}$ [357]. Similar to above research, Pd functionalized $\mathrm{SnO}_{2}$ nanofibers [366] used in the discrimination of butane at $260^{\circ} \mathrm{C}$ (see Table 5), but its sensor response needs to be improved with further investigations. As an inclusion to the VOC sensory research, nanotubes (Pt-decorated CNTs, $3 \mathrm{D} \mathrm{TiO} 2 / \mathrm{G}-\mathrm{CNTs}$ and hierarchical $\mathrm{NiCo}_{2} \mathrm{O}_{4}$ NTs) were demonstrated towards toluene or xylene discrimination [367-369]. As seen in Table 5, $\mathrm{TiO}_{2}$ NPs decorated G-CNTs seems to be an excellent candidate towards toluene detection at room temperature with a calculated LOD of $0.4 \mathrm{ppm}$ [368].

Nanobelts (Fe doped $\mathrm{MoO}_{3}$ nanobelts and $\mathrm{Au}$ decorated $\mathrm{ZnO} / \mathrm{In}_{2} \mathrm{O}_{3}$ belt-tooth nanostructure), nanocages ( $\mathrm{ZnO} / \mathrm{ZnCo}_{2} \mathrm{O}_{4}$ hollow nanocages), nanosheets (Au functionalized $\mathrm{WO}_{3} \cdot \mathrm{H}_{2} \mathrm{O}$ NShs, porous h-BN 3D NShs, and Nb-doped NiO NShs), nanoflakes (CdO hexagonal nanoflakes and $\mathrm{ZnO}-\mathrm{CeO}_{2}$ triangular nanoflakes), and nanospheres $\left(\mathrm{ZnFe}_{2} \mathrm{O}_{4}\right.$ NSPs and Pt doped $\mathrm{CoCr}_{2} \mathrm{O}_{4}$ hollow NSPs) were demonstrated to volatile hydrocarbons quantitation by the researchers as noted in Table 5 [370-379]. Among them, Nb-doped $\mathrm{NiO}$ NShs and $\mathrm{CoCr}_{2} \mathrm{O}_{4}$ hollow NSPs [375,379] are highly notable with their sensor responses to xylene vapor $\left(\mathrm{R}_{\mathrm{a}} / \mathrm{R}_{\mathrm{g}}=335.1\right.$ for $100 \mathrm{ppm}$ at $370{ }^{\circ} \mathrm{C}$ and $\mathrm{R}_{\mathrm{a}} / \mathrm{R}_{\mathrm{g}}=559$ for $5 \mathrm{ppm}$ at $275^{\circ} \mathrm{C}$, respectively) with LODs down to sub-ppm level (0.002 ppm and $0.0187 \mathrm{ppm}$, correspondingly). In light of this, involvement of porous nanostructures is anticipated due to the high adsorption nature through the pores [374]. Yao et al. discussed the pore-effect of $\mathrm{Pd}_{-} \mathrm{SnO}_{2}$ nanoporous composite [380] for the capture and sensing of methane gas as illustrated in Figure 23. The material shows reasonable sensor response (see Table 5) in methane sensory research. In view of this, p-n $\mathrm{Co}_{3} \mathrm{O}_{4}-\mathrm{TiO}_{2}$ mesoporous hierarchical nanostructures were elaborated in the sensing of xylene at an operating temperature of $115^{\circ} \mathrm{C}$ [381]. In which, the response is well enough $\left(R_{a} / R_{g}=113\right.$ for $50 \mathrm{ppm}$; response/recovery time $=130 \mathrm{~s} / 150 \mathrm{~s}$ ) due to the pore-effect of the material. In parallel to utilization of distinct nanostructures in volatile hydrocarbons discriminations, many hierarchical nanomaterials were also employed as described below. Hierarchical nanostructures like Au loaded $\mathrm{MoO}_{3}$ hollow NSPs, $\mathrm{Pt}-\mathrm{SnO}_{2}$ hollow NSPs, $\mathrm{NiO} / \mathrm{NiMoO}_{4} \mathrm{NSPs}, \mathrm{Co}_{3} \mathrm{O}_{4}$, and $\mathrm{WO}_{3}$ were demonstrated in the sensing of toluene, xylene, methane, or acetylene at various temperature $\left(180-340{ }^{\circ} \mathrm{C}\right)$ as noted in Table 5 [382-386]. Among them, hierarchical nanostructure of $\mathrm{Co}_{3} \mathrm{O}_{4}$ displayed a sensor response to toluene at $180^{\circ} \mathrm{C}$ even after one month, thereby is noted as a reliable system [385]. In this work, authors described the sensory performance of cube shaped $\mathrm{Co}_{3} \mathrm{O}_{4}\left(\mathrm{C}-\mathrm{Co}_{3} \mathrm{O}_{4}\right)$, rod shaped $\mathrm{Co}_{3} \mathrm{O}_{4}\left(\mathrm{R}-\mathrm{Co}_{3} \mathrm{O}_{4}\right)$, and sheet shaped (S$\mathrm{Co}_{3} \mathrm{O}_{4}$ ) nanostructures synthesized via hydrothermal tactic. Wherein, sheet shaped (S$\mathrm{Co}_{3} \mathrm{O}_{4}$ ) hierarchical nanostructure showed a higher response than that of others as shown in Figure 24. 
(a)

- $\mathrm{SnO}_{2} \cdot \mathrm{Pd} \mathrm{CH}_{4}$

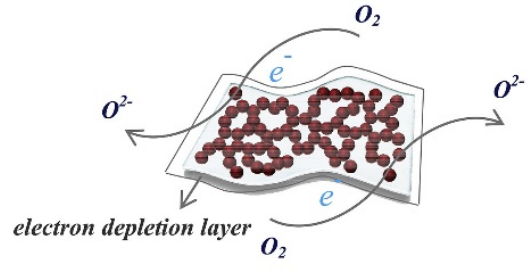

In air

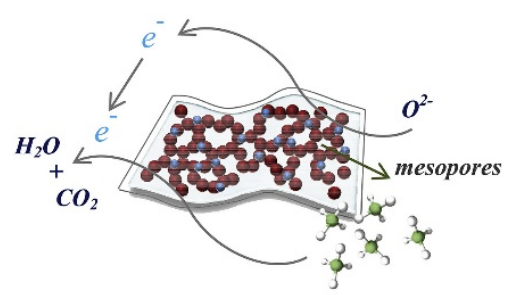

In methane

(b)
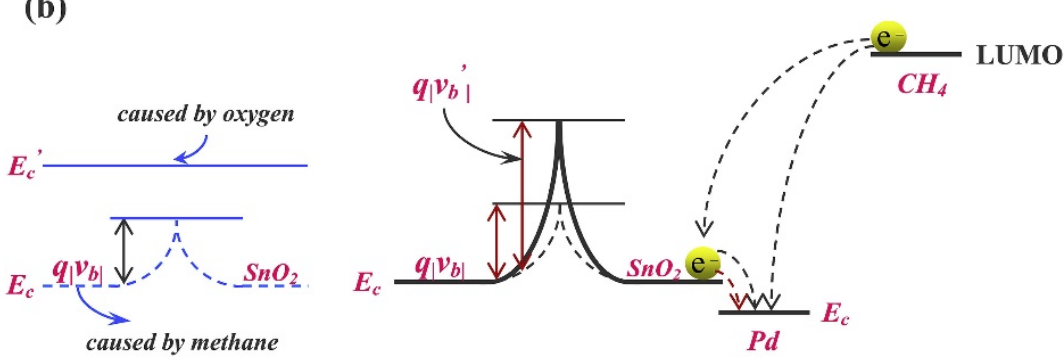

Figure 23. $(\mathbf{a}, \mathbf{b})$ Schematic diagram of the sensing reaction mechanism of the $\mathrm{Pd}_{-} \mathrm{SnO}_{2}$ composite nanoporous structure (Reproduced with the permission from Ref. [380]).
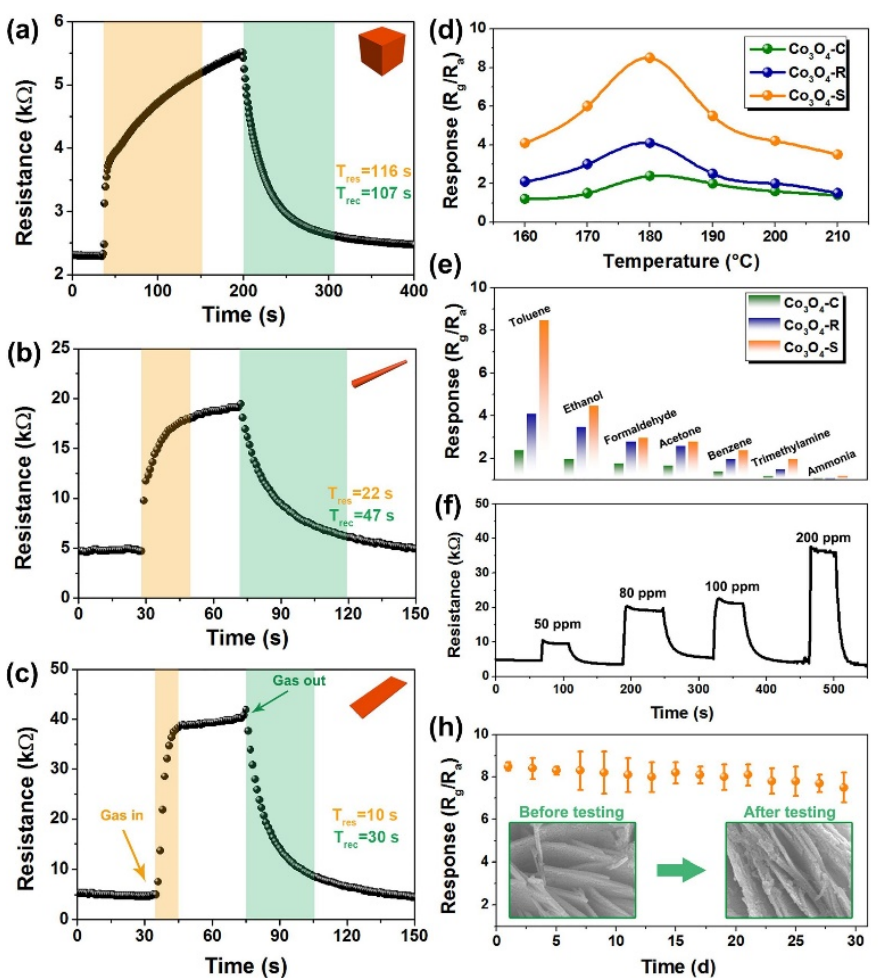
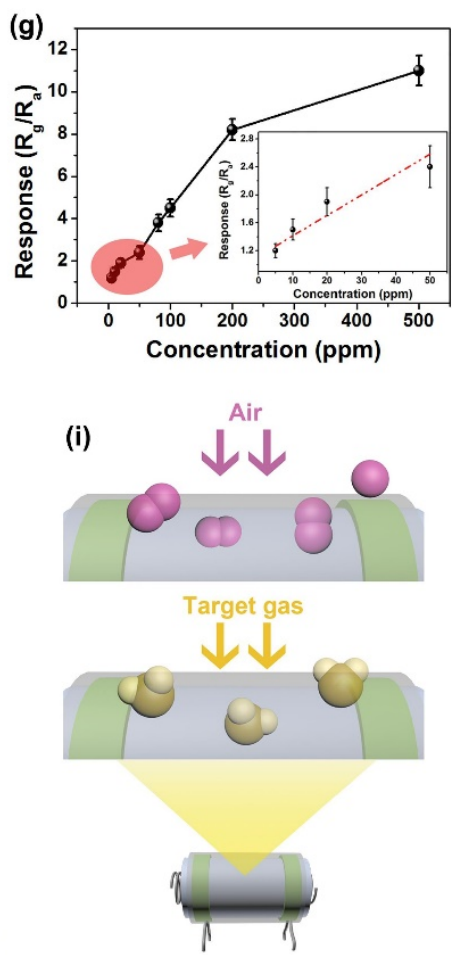

Figure 24. Resistance curves of the sensors based on (a) $\mathrm{Co}_{3} \mathrm{O}_{4}-\mathrm{C}$, (b) $\mathrm{Co}_{3} \mathrm{O}_{4}-\mathrm{R},(\mathbf{c}) \mathrm{Co}_{3} \mathrm{O}_{4}-\mathrm{S}$ towards $200 \mathrm{ppm}$ toluene at $180{ }^{\circ} \mathrm{C}$, respectively; (d) responses of three sensors towards $200 \mathrm{ppm}$ toluene at different working temperatures; (e) responses of three sensors to $200 \mathrm{ppm}$ different target gases at $180{ }^{\circ} \mathrm{C}$; (f) dynamic resistance curve of the sensor based on $\mathrm{Co}_{3} \mathrm{O}_{4}$-S to different concentration of toluene; (g) relationship between response and toluene concentration; (h) response and morphology stability of $\mathrm{Co}_{3} \mathrm{O}_{4}$-S-based sensor to $200 \mathrm{ppm}$ toluene during 30 days (measurement number $=3$ ); (i) schematic of sensor exposed to air and target gas (Reproduced with the permission from Ref. [385]). 
In addition, $\mathrm{PbS}$ NPs decorated $\mathrm{CdO}$ necklace like nanobeads were discussed in the quantitation of LPG gas at RT by Sonawane and co-workers [387]. Nevertheless, further investigation is necessary to authenticate the material's reliability. In light of this, $\mathrm{Au}$ loaded $\mathrm{TiO}_{2}$ hedgehog-like nanostructure was proposed to detect xylene at $375{ }^{\circ} \mathrm{C}$, thereby can be included as an additional candidate [388]. These materials were synthesized by hydrothermal method followed by simple isometric impregnation route. They are required to be optimized to minimize the working temperature during the sensor studies.

Similar to microstructure-based volatile hydrocarbon vapors quantification (example: $\mathrm{Sn}^{2+}$ doped $\mathrm{NiO}$ microspheres in Xylene detection with sub-ppm LOD) [389], nanocomposites, such as $\mathrm{Pd} / \mathrm{PdO} / \mathrm{S}-\mathrm{SnO}_{2}$ nanocomposite film, $\mathrm{rGO} / \mathrm{Co}_{3} \mathrm{O}_{4}$ nanocomposite, $\mathrm{WO}_{3}$ decorated $\mathrm{TiO}_{2} \mathrm{NPs}$ nanocomposite, $\mathrm{BGQD} / \mathrm{Ag}-\mathrm{LaFeO} 3$ nanocomposite, $\mathrm{Ag} / \mathrm{Bi}_{2} \mathrm{O}_{3}$ nanocomposite, $\mathrm{AgO}$ loaded $\mathrm{LaFeO}_{3}$ nanocomposite, $\mathrm{CuO} \mathrm{NPs}-\mathrm{Ti}_{3} \mathrm{C}_{2} \mathrm{Tx}$ MXene nanocomposite, and Graphene $/ \mathrm{SnO}_{2}$ NPs nanocomposite were effectively applied in the detection of hydrocarbons as detailed in Table 5 [390-397]. Wherein, $\mathrm{Ag} / \mathrm{Bi}_{2} \mathrm{O}_{3}$ nanocomposite and Graphene $/ \mathrm{SnO}_{2} \mathrm{NPs}$ nanocomposite were found to perform remarkably in terms of their operation temperature (at room temperature) [394,397]. Recently, Luo et al. described the sensitivity of a polymer-SWCNTs composite toward BTX with an exceptional LOD of 5 ppm [398]. However, this work also depends on the polymer properties and requires more detailed investigations.

Table 5. Detection concentration, response/recovery time, operating temperature (Temp.), and LODs to volatile hydrocarbons gases by diverse nanostructured materials.

\begin{tabular}{|c|c|c|c|c|c|c|}
\hline Materials/Nanostructure & Analyte/Concentration & $\begin{array}{c}\text { Gas Response } \\
\left(\mathbf{R}_{\text {air }} / \mathbf{R}_{\text {gas }}\right)\end{array}$ & Response/Recovery & Temp. & LOD & Ref \\
\hline $\mathrm{Ag}-\mathrm{LaFeO}_{3} /$ nanoparticles & Xylene/5 ppm & 36.2 & $114 \mathrm{~s} / 55 \mathrm{~s}$ & $99^{\circ} \mathrm{C}$ & $<1 \mathrm{ppm}$ & [352] \\
\hline $\mathrm{Ag}-\mathrm{LaFeO}_{3} /$ nanoparticles & Xylene/10 ppm & 16.76 & $68 \mathrm{~s} / 36 \mathrm{~s}$ & $125^{\circ} \mathrm{C}$ & $0.2 \mathrm{ppm}$ & [353] \\
\hline $\mathrm{Au}-\mathrm{ZnO} /$ nanoparticles & Xylene/100 ppm & 92 & $4 \mathrm{~s} / 6 \mathrm{~min}$ & $377^{\circ} \mathrm{C}$ & NA & [354] \\
\hline $\begin{array}{c}\text { cobalt porphyrin } \\
(\mathrm{CoPP}) \text {-functionalized } \\
\mathrm{TiO}_{2} / \text { nanoparticles }\end{array}$ & $\begin{array}{l}\text { Benzene, Toluene and } \\
\text { Xylene (BTX)/10 ppm }\end{array}$ & $>5$ & $40 \mathrm{~s} / 80 \mathrm{~s}$ & $240^{\circ} \mathrm{C}$ & $0.005 \mathrm{ppm}$ & [355] \\
\hline In-doped $\mathrm{ZnO} /$ Quantum dots & Acetylene/10 ppm & 19.3 & $\sim 100 \mathrm{~s} / \mathrm{NA}$ & $400^{\circ} \mathrm{C}$ & $0.1 \mathrm{ppm}$ & [356] \\
\hline $\begin{array}{c}\text { Metal organic } \\
\text { Frameworks/nanocrystals }\end{array}$ & $\begin{array}{l}\text { Benzene, Toluene, Ethyl } \\
\text { benzene and Xylene } \\
\text { (BTEX)/50 ppm }\end{array}$ & $>20$ & NA & $\begin{array}{l}\text { Room } \\
\text { Temp. }\end{array}$ & $0.4 \mathrm{ppm}$ & [357] \\
\hline$\alpha-\mathrm{Fe}_{2} \mathrm{O}_{3} / \mathrm{SnO}_{2} /$ nanowire arrays & Toluene/100 ppm & 49.7 & $20 \mathrm{~s} / 15 \mathrm{~s}$ & $90^{\circ} \mathrm{C}$ & $\sim 50 \mathrm{ppm}$ & [358] \\
\hline 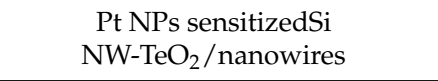 & Toluene/50 ppm & 45 & $20 \mathrm{~s} / 500 \mathrm{~s}$ & $200^{\circ} \mathrm{C}$ & $\sim 10 \mathrm{ppm}$ & [359] \\
\hline CoPP-ZnO/nanorods & Toluene/10 ppm & $>2.5$ & NA & NA & $0.002 \mathrm{ppm}$ & [361] \\
\hline$\alpha-\mathrm{MoO}_{3} /$ nanoarrays & Xylene/100 ppm & 19.2 & $1 \mathrm{~s} / \leq 20 \mathrm{~s}$ & $370^{\circ} \mathrm{C}$ & $\sim 10 \mathrm{ppm}$ & [362] \\
\hline $\mathrm{Y}$ doped $\alpha-\mathrm{MoO}_{3} /$ nanoarrays & Xylene/100 ppm & 28.3 & $1 \mathrm{~s} / \sim 15 \mathrm{~s}$ & $370^{\circ} \mathrm{C}$ & $\sim 5 \mathrm{ppm}$ & [363] \\
\hline $\begin{array}{l}\text { MOF-driven metal- embedded } \\
\text { metal oxide (Pd@ZnO- } \\
\left.\mathrm{WO}_{3}\right) / \text { nanofibers }\end{array}$ & Toluene/1 ppm & 22.22 & $<20 \mathrm{~s} / \mathrm{NA}$ & $350^{\circ} \mathrm{C}$ & $0.1 \mathrm{ppm}$ & [364] \\
\hline $\mathrm{V}_{2} \mathrm{O}_{5} /$ nanofibers & Xylene/500 ppm & 191 & $80 \mathrm{~s} / 50 \mathrm{~s}(100 \mathrm{ppm})$ & $\begin{array}{l}\text { Room } \\
\text { Temp. }\end{array}$ & $\sim 5 \mathrm{ppm}$ & [365] \\
\hline $\begin{array}{l}\mathrm{Pd} \text { functionalized } \\
\mathrm{SnO}_{2} / \text { nanofibers }\end{array}$ & Butane/3000 ppm & 47.58 & $3.20 \mathrm{~s} / 6.28 \mathrm{~s}$ & $260^{\circ} \mathrm{C}$ & $\sim 10 \mathrm{ppm}$ & [366] \\
\hline Pt-decorated CNTs/nanotubes & Toluene/5 ppm & 5.06 & $90 \mathrm{~s} / 520 \mathrm{~s}$ & $150^{\circ} \mathrm{C}$ & $\sim 1 \mathrm{ppm}$ & [367] \\
\hline $3 \mathrm{D} \mathrm{TiO}_{2} / \mathrm{G}-\mathrm{CNT} /$ nanotubes & Toluene/500 ppm & $42.9 \%$ & 9-11 s (for both) & $\begin{array}{l}\text { Room } \\
\text { Temp. }\end{array}$ & $0.4 \mathrm{ppm}$ & [368] \\
\hline $\mathrm{NiCo}_{2} \mathrm{O}_{4} /$ nanotubes (hierarchical) & Xylene/100 ppm & 9.25 & $20 \mathrm{~s} / 9 \mathrm{~s}$ & $220^{\circ} \mathrm{C}$ & $\sim 1 \mathrm{ppm}$ & [369] \\
\hline Fe doped $\mathrm{MoO}_{3} /$ nanobelts & Xylene/100 ppm & 6.1 & $20 \mathrm{~s} / 75 \mathrm{~s}$ & $206^{\circ} \mathrm{C}$ & $\sim 5 \mathrm{ppm}$ & [370] \\
\hline $\begin{array}{c}\mathrm{Au} \text { decorated } \\
\mathrm{ZnO} / \mathrm{In}_{2} \mathrm{O}_{3} \text { /belt-tooth } \\
\text { nanostructure }\end{array}$ & Acetylene/100 ppm & 5 & $8.5 \mathrm{~s} / \mathrm{NA}$ & $90^{\circ} \mathrm{C}$ & $\sim 25 \mathrm{ppm}$ & [371] \\
\hline
\end{tabular}


Table 5. Cont.

\begin{tabular}{|c|c|c|c|c|c|c|}
\hline Materials/Nanostructure & Analyte/Concentration & $\begin{array}{l}\text { Gas Response } \\
\left(\mathbf{R}_{\text {air }} / \mathbf{R}_{\text {gas }}\right)\end{array}$ & Response/Recovery & Temp. & LOD & Ref \\
\hline $\mathrm{ZnO} / \mathrm{ZnCo}_{2} \mathrm{O}_{4} /$ hollow nanocages & Xylene/100 ppm & 34.26 & NA & $320^{\circ} \mathrm{C}$ & $0.126 \mathrm{ppm}$ & [372] \\
\hline $\begin{array}{l}\mathrm{Au} \\
\text { functionalized } \mathrm{WO}_{3} \cdot \mathrm{H}_{2} \mathrm{O} / \text { nanosheets }\end{array}$ & Toluene/100 ppm & 50 & $2 \mathrm{~s} / 9 \mathrm{~s}$ & $300^{\circ} \mathrm{C}$ & $\sim 10 \mathrm{ppm}$ & [373] \\
\hline $\mathrm{Nb}$-doped $\mathrm{NiO} /$ nanosheets & Xylene/100 ppm & 335.1 & $63 \mathrm{~s} / 66 \mathrm{~s}$ & $370^{\circ} \mathrm{C}$ & $0.002 \mathrm{ppm}$ & [375] \\
\hline $\mathrm{CdO} /$ hexagonal nanoflakes & $\begin{array}{l}\text { liquefied petroleum gas } \\
\text { (LPG)/500 ppm }\end{array}$ & $\sim 27.5$ & $8.6 \mathrm{~s} / 10 \mathrm{~s}$ & $270^{\circ} \mathrm{C}$ & $\sim 10 \mathrm{ppm}$ & [376] \\
\hline $\mathrm{ZnO}-\mathrm{CeO}_{2} /$ triangular nanoflakes & $\mathrm{BTEX} / 50 \mathrm{ppm}$ & $10-21$ & $8 \mathrm{~s} / 10 \mathrm{~s}$ & $200^{\circ} \mathrm{C}$ & $0.01 \mathrm{ppm}$ & [377] \\
\hline $\mathrm{ZnFe}_{2} \mathrm{O}_{4} /$ nanospheres & Toluene/100 ppm & 9.98 & $18.14 \mathrm{~s} / 29.2 \mathrm{~s}$ & $300^{\circ} \mathrm{C}$ & $\sim 1 \mathrm{ppm}$ & [378] \\
\hline $\begin{array}{c}\text { Pt doped } \mathrm{CoCr}_{2} \mathrm{O}_{4} / \text { hollow } \\
\text { nanospheres }\end{array}$ & Xylene/5 ppm & 559 & $300 \mathrm{~s} / 600 \mathrm{~s}$ & $275^{\circ} \mathrm{C}$ & $0.0187 \mathrm{ppm}$ & [379] \\
\hline $\mathrm{Pd}_{-} \mathrm{SnO}_{2} /$ nanoporous composite & Methane/3000 ppm & 17.6 & $3 \mathrm{~s} / 5 \mathrm{~s}$ & $340^{\circ} \mathrm{C}$ & $\sim 100 \mathrm{ppm}$ & [380] \\
\hline $\begin{array}{l}\mathrm{Co}_{3} \mathrm{O}_{4}-\mathrm{TiO}_{2} / \text { mesoporous } \\
\text { hierarchical nanostructure }\end{array}$ & Xylene/50 ppm & 113 & $130 \mathrm{~s} / 150 \mathrm{~s}$ & $115^{\circ} \mathrm{C}$ & $\sim 5 \mathrm{ppm}$ & [381] \\
\hline $\begin{array}{l}\text { Au loaded } \mathrm{MoO}_{3} / \text { hollow } \\
\text { nanospheres (hierarchical) }\end{array}$ & $\begin{array}{c}\text { Toluene and Xylene } / 100 \\
\text { ppm }\end{array}$ & $17.5 \& 22.1$ & $19 \mathrm{~s} / 6 \mathrm{~s} \& 1.6 \mathrm{~s} / 2 \mathrm{~s}$ & $250^{\circ} \mathrm{C}$ & $0.1 \& 0.5 \mathrm{ppm}$ & [382] \\
\hline $\begin{array}{l}\mathrm{Pt}^{-S_{n O}} \mathrm{O}_{2} \text { hollow nanospheres } \\
\text { (hierarchical) }\end{array}$ & Methane/250 ppm & $4.88 \& 4.33$ & NA & $\begin{array}{c}300^{\circ} \mathrm{C} \& \\
340{ }^{\circ} \mathrm{C}\end{array}$ & $\sim 25 \mathrm{ppm}$ & [383] \\
\hline $\begin{array}{c}\mathrm{NiO} / \mathrm{NiMoO}_{4} / \text { hierarchical } \\
\text { nanospheres }\end{array}$ & $p$-Xylene/5 ppm & 101.5 & $10-50 \mathrm{~s} / 20-200 \mathrm{~s}$ & $375^{\circ} \mathrm{C}$ & $0.02 \mathrm{ppm}$ & [384] \\
\hline $\mathrm{Co}_{3} \mathrm{O}_{4} /$ hierarchical nanostructure & Toluene/200 ppm & 8.5 & $10 \mathrm{~s} / 30 \mathrm{~s}$ & $180^{\circ} \mathrm{C}$ & $\sim 5 \mathrm{ppm}$ & [385] \\
\hline $\mathrm{WO}_{3} /$ hierarchical nanostructure & Acetylene/200 ppm & 32.31 & $12 \mathrm{~s} / 17 \mathrm{~s}$ & $275^{\circ} \mathrm{C}$ & $<5 \mathrm{ppm}$ & [386] \\
\hline $\begin{array}{l}\mathrm{PbS} \text { NPs decorated } \mathrm{CdO} / \text { necklace } \\
\text { like nanobeads }\end{array}$ & LPG/1176 ppm & $\sim 50$ & $148 \mathrm{~s} / 142 \mathrm{~s}$ & $\begin{array}{l}\text { Room } \\
\text { Temp. }\end{array}$ & $\sim 300$ ppm & [387] \\
\hline $\begin{array}{l}\text { Au loaded } \mathrm{TiO}_{2} / \text { hedgehog-like } \\
\text { nanostructure }\end{array}$ & Xylene/100 ppm & 6.49 & $5 \mathrm{~s} / 2 \mathrm{~s}$ & $375^{\circ} \mathrm{C}$ & $\sim 2 \mathrm{ppm}$ & [388] \\
\hline $\begin{array}{l}\mathrm{Pd} / \mathrm{PdO} / \mathrm{S}-\mathrm{SnO}_{2} / \text { nano- } \\
\text { composite film }\end{array}$ & methane/300 ppm & 12.3 & $8 \mathrm{~s} / 12 \mathrm{~s}$ & $240^{\circ} \mathrm{C}$ & $<50$ ppm & [390] \\
\hline $\mathrm{rGO} / \mathrm{Co}_{3} \mathrm{O}_{4}$ /nano- composite & Toluene/5 ppm & 11.3 & NA & $110^{\circ} \mathrm{C}$ & $\geq 0.5 \mathrm{ppm}$ & [391] \\
\hline $\begin{array}{l}\mathrm{WO}_{3} \text { decorated } \mathrm{TiO}_{2} \mathrm{NPs} / \text { nano- } \\
\text { composite }\end{array}$ & Xylene/10 ppm & 92.53 & $410 \mathrm{~s} / 2563 \mathrm{~s}$ & $160^{\circ} \mathrm{C}$ & $1 \mathrm{ppm}$ & [392] \\
\hline $\begin{array}{l}\mathrm{BGQD} / \mathrm{Ag}-\mathrm{LaFeO}_{3} / \text { nano- } \\
\text { composite }\end{array}$ & Benzene/1 ppm & 17.5 & NA & $65^{\circ} \mathrm{C}$ & $<1 \mathrm{ppm}$ & [393] \\
\hline $\mathrm{Ag} / \mathrm{Bi}_{2} \mathrm{O}_{3} /$ nano- composite & Toluene/50 ppm & $89.2 \%$ & NA & $\begin{array}{l}\text { Room } \\
\text { Temp. }\end{array}$ & $\sim 10$ ppm & [394] \\
\hline $\begin{array}{l}\mathrm{AgO} \text { loaded } \mathrm{LaFeO}_{3} / \text { nano- } \\
\text { composite }\end{array}$ & Acetylene/100 ppm & 60 & $6.1 \mathrm{~min} / 4.7 \mathrm{~min}$ & $200^{\circ} \mathrm{C}$ & $\sim 5 \mathrm{ppm}$ & [395] \\
\hline $\begin{array}{l}\mathrm{CuONPs}-\mathrm{Ti}_{3} \mathrm{C}_{2} \mathrm{TxMXene} / \text { nano- } \\
\text { composite }\end{array}$ & Toluene/50 ppm & 11.4 & $270 \mathrm{~s} / 10 \mathrm{~s}$ & $250^{\circ} \mathrm{C}$ & $0.32 \mathrm{ppm}$ & [396] \\
\hline $\begin{array}{l}\text { Graphene } / \mathrm{SnO}_{2} \mathrm{NPs} \\
\text { nano-composite }\end{array}$ & BTX/0.2-11 ppm & $1-28$ & NA & $\begin{array}{l}\text { RT and } \\
250^{\circ} \mathrm{C}\end{array}$ & $\sim 0.2 \mathrm{ppm}$ & [397] \\
\hline
\end{tabular}

NA $=$ Not available; Temp. $=$ Temperature; $\mathrm{s}=$ Seconds $; \min =$ minutes.

\section{Nanostructures in other VOCs Determinations}

Apart from the quantitation of acetone, alcohols, aldehydes, amines, and hydrocarbons, nanomaterials were also consumed in the discrimination of other VOCs as detailed in this section. For instance, Ma et al. reported the acetic acid sensing properties of Co-doped $\mathrm{LaFeO}_{3}$ nanofibers [399]. The fabricated nanofibers detected the acetic acid at $130{ }^{\circ} \mathrm{C}$ than that of other interferences (ethanol, methanol, acetone, $\mathrm{N}, \mathrm{N}^{\prime}$-dimethylformamide (DMF), ammonia, and benzene). This work requires further investigations to identify various sensory details. Mesoporous tungsten oxides with crystalline framework were utilized in the highly selective detection of foodborne pathogen "3-hydroxy-2-butanone" and proposed in 
food safety by Zhu and co-workers [400]. Compared to all other competing species at high concentration (50 ppm), the material displayed a high response to 3-hydroxy-2-butanone at low concentration ( $\mathrm{R}_{\mathrm{a}} / \mathrm{R}_{\mathrm{g}} \geq 25$ for $5 \mathrm{ppm}$ ) at $290^{\circ} \mathrm{C}$ with response/recovery time of $<1 \mathrm{~min}$ and a LOD of $0.1 \mathrm{ppm}$. The porosity of this material plays a vital role in the sensory studies with similar mechanism proposed in acetone. This is an excellent and representative work in food safety. Aqueous electrochemical-based detection of chloroform was proposed by Hamid and co-workers [401]. Wherein, $\mathrm{Fe}_{2} \mathrm{O}_{3}$ NPs decorated $\mathrm{ZnO}$ NRds were used in this work, which showed linear response from $10 \mu \mathrm{M}$ to $10 \mathrm{mM}$ with a calculated LOD of $0.6 \mu \mathrm{M}$. However, this material needs to be investigated to optimize the resistance-based sensor responses. For example, MOF derived core-shell $\mathrm{PrFeO}_{3}$ functionalized $\alpha-\mathrm{Fe}_{2} \mathrm{O}_{3}$ nano-octahedrons were employed in the quantitation of ethyl acetate at $206^{\circ} \mathrm{C}$ as seen in Figure 25 [402]. For 100 ppm ethyl acetate, the sensor response reached 22.85 with response/recovery time of $<15 \mathrm{~s}$ with LOD down to $1 \mathrm{ppm}$. Therefore, this work is noted as an excellent one towards ethyl acetate discrimination in the presence of competing species (ethanol, acetone, xylene, formaldehyde, and benzene).
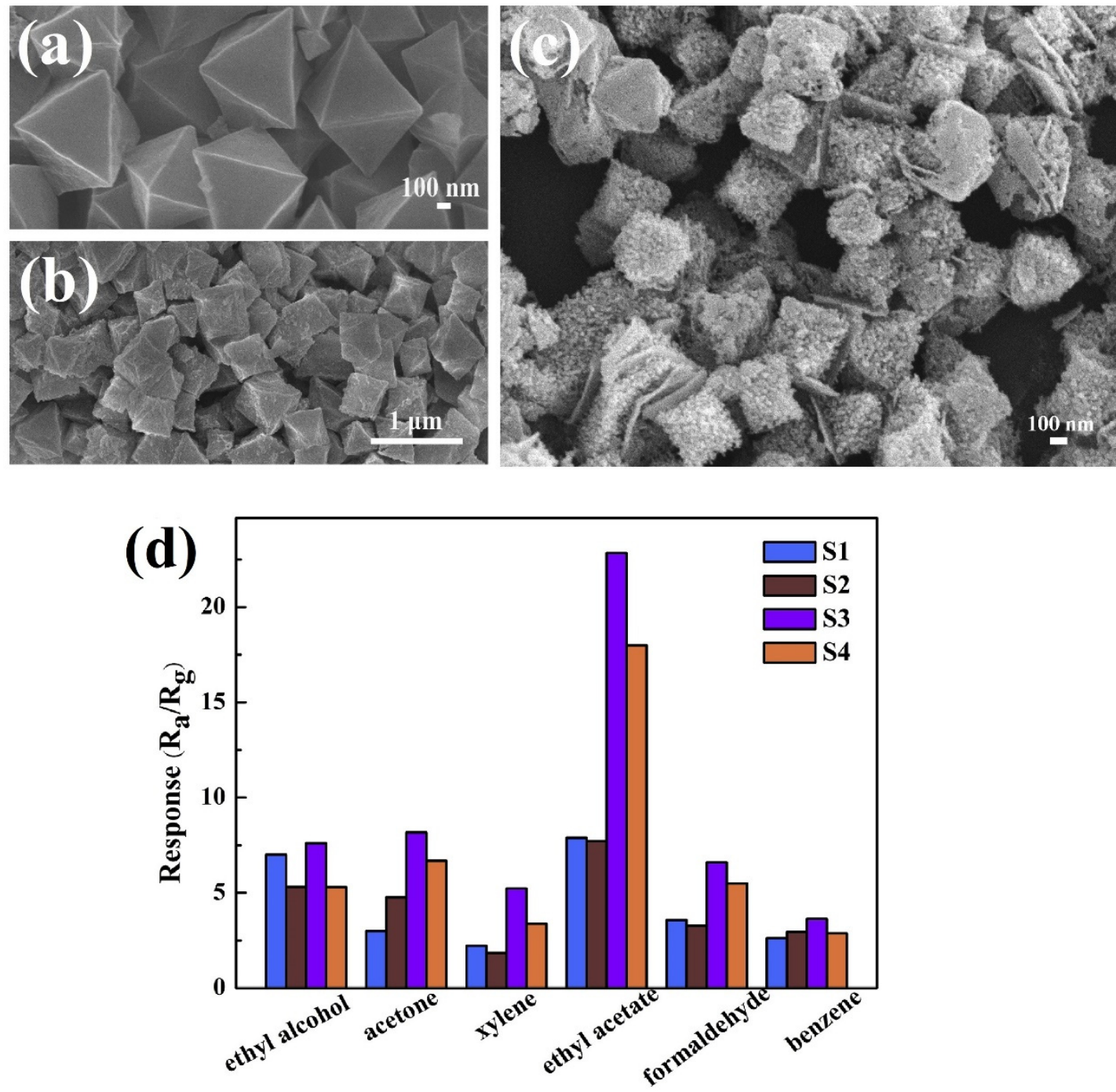

Figure 25. (a) Typical FESEM images of MIL-53 nano-octahedrons; (b) SEM images of MIL-53/Pr-Fe hydroxide precursor; (c) typical FESEM image of pure core-shell $\alpha$ - $\mathrm{Fe}_{2} \mathrm{O}_{3}$ nano-octahedrons; (d) Selectivity of four sensors upon exposure to $100 \mathrm{ppm}$ various interfering gases at $255^{\circ} \mathrm{C}$ (S1) and $206^{\circ} \mathrm{C}(\mathrm{S} 2-\mathrm{S} 4)$, respectively, (reproduced with the permission from Ref. [402]). 
Gao and his research team discussed the detection ability of $\mathrm{ZnO}$ NRds bundles towards three kinds of abused drugs $\left(\mathrm{C}_{10} \mathrm{H}_{15} \mathrm{NO}, \mathrm{C}_{9} \mathrm{H}_{13} \mathrm{NO}\right.$ and $\left.\mathrm{C}_{8} \mathrm{H}_{9} \mathrm{NO}_{2} ; 100 \mathrm{ppm}\right)$ at $252{ }^{\circ} \mathrm{C}$ via the similar mechanism in VOCs detection [403]. These $\mathrm{ZnO}$ NRds bundles were synthesized by hydrothermal method, and upon exposure to those abused drugs vapors, the sensor responses were higher $(>40)$ than that of other interferences. Firstly, the n-type $\mathrm{ZnO}$ NRds bundles adsorb $\mathrm{O}_{2}$ molecules in air to capture free electrons in conduction band (at the device surface) and convert them to adsorbed oxygen species $\left(\mathrm{O}^{2-}, \mathrm{O}_{2}{ }^{-}, \mathrm{O}^{-}\right)$. Secondly, decrease in the number of electrons in the conduction band leads to the formation of a depletion layer, which increases resistance in $\mathrm{ZnO}$. Consequently, the electrons are released back to conduction band upon interaction with target drug vapors, which affects the depletion layer and the resistance of $\mathrm{ZnO}$. The changes in resistance can provide sensor responses of interactions with target drug vapor. Moreover, this work performed quite well at high humid condition ( $>90 \% \mathrm{RH})$. In a similar fashion, $\mathrm{Ag}-\mathrm{ZnO}-\mathrm{SWCNT}$ field effect transistor (FET) device demonstrated its sensing performance to organophosphorus pesticide "methyl parathion" with linear response from $1 \times 10^{-16}$ to $1 \times 10^{-4} \mathrm{M}$ and a LOD of $0.27 \times 10^{-16} \mathrm{M}$ [404]. The response and recovery time of this sensor is $1 \mathrm{~s}$ and $3 \mathrm{~s}$, respectively. Moreover, this work also attested by real time analysis in rice and soil, thereby is noted as good innovation.

A FET device based on electrospun InYbO nanofibers was proposed towards the sensing of DMF at room temperature by Chen and co-workers [405]. The sensor showed remarkable response $\left(R_{a} / R_{g}=89\right)$ to $2 \mathrm{ppm} D M F$, with an estimated LOD of $0.00118 \mathrm{ppm}$. Moreover, it delivered fast response/recovery time of $36 \mathrm{~s} / 67 \mathrm{~s}$, thereby is noted as encouraging research with a wide scope. In view of this, $\mathrm{MgGa}_{2} \mathrm{O}_{4}$ /graphene composites were synthesized by simple hydrothermal route and utilized in the sensing of acetic acid at room temperature [406]. At $0.1 \mathrm{wt} \%$ graphene, the material displayed a high sensor response $\left(\mathrm{R}_{\mathrm{a}} / \mathrm{R}_{\mathrm{g}}=363.3\right.$ for $100 \mathrm{ppm}$; response $/$ recovery time $\left.=50 \mathrm{~s} / 35 \mathrm{~s}\right)$ with a calculated LOD of $0.001 \mathrm{ppm}$. Therefore, it is noted as an exceptional material and can be consumed in acetic acid sensory studies in future.

\section{Advantages and Limitations}

Consumption of distinct nanostructured materials in VOCs detection also has a few advantages and limitations as noted below.

1. By tuning the nanostructural features, materials with similar compositions are able to detect diverse VOC analytes.

2. Modulation of nanostructures might be able to tune the availability of surface area to enhance adsorption of VOC analytes, thereby the sensor response can be improved significantly.

3. By adopting different nanostructures, the operating temperature for the sensing of specified VOC can be reduced or lowered to room temperature.

4. Divergent nanostructures formation via the combination of diverse materials and their decoration or doping or functionalization may enhance/tune the conducting and charge/electron transport properties, which can help the device to detect specific VOC from other competing species.

5. Utilization of known and easily fabricated semiconducting materials with diverse nanostructures may led to the generation of cost-effective and reliable devices with social impact.

6. The less toxic nature of some semiconducting materials is noted as an advantage and can be implemented in upcoming health care devices.

7. Synthesis of majority of diverse nanostructures seems to be more complicated with involvement of multiple synthetic tactics, such as hydrothermal, CVD, impregnation, electrospinning, etc. The requirement of processing at high temperature further increases the production cost.

8. Reliability of numerous diverse semiconducting nanostructures to specific VOC is still in question due to the higher operating temperature. 
9. Reports on temperature dependent multiple analyte sensors by a few nanostructured materials are still not convincing with the interference studies; therefore, applicability of those materials is limited and requires more interrogations.

10. Fabrication of diverse nanostructured materials is also limited by the sophisticated clean room atmosphere and characterizations using costly equipment, such as SEM, TEM, electrochemical instruments, etc.

11. Majority of reported nanostructures are still not authenticated by real time applications, which limits the operation of those devices in VOC detection.

12. In general, porous nanostructures display the high/low responses to VOCs due to their pore-effect but are limited in operation by their uneven results.

13. Stability of sensory reports is considerably affected by high humid conditions and is restricted in real time applications.

\section{Conclusions and Perspectives}

This review gives a concise summary of the diverse nanostructured material-based detection of VOCs (more than 340 references that have been published since 2016), such as acetone, alcohols, aldehydes, amines, hydrocarbons, and other volatile organic compounds. The underlying mechanism of those temperature tuned semiconducting device-based assays of VOCs is also discussed in brief with given advantages and limitations. Moreover, the VOC sensory utilities of diverse nanostructures and nanocomposites with $p-p, p-n, n-n$ heterojunction structures are also presented. Wherein, numerous inorganic-nanostructured materials such as metal oxides, perovskites and composited structures are currently noted as effective candidates towards VOCs detection. Further, majority of reports mainly follows the similar trend to detect the aforementioned VOCs. In addition to those sensory details, following points also require much attention.

1. Synthetic complications on the development of semiconducting materials towards VOCs sensors must be reduced with respect to cost-effect and reliability.

2. Justification regarding the role of semiconducting property in the sensing studies seems to be deficient in some reports, which requires more investigations.

3. Majority of the reports did not provide any theoretical or in-depth explanation regarding, "Why the attested nanostructure becomes more specific to the certain target?" Therefore, extensive research must be done in future.

4. Utilization of diverse nanostructures with similar material compositions towards different VOCs still needs more interpretations.

5. Numerous VOCs sensors operate at higher temperature, thereby optimization research should be conducted to make the devices operable at low temperature or even room temperature.

6. Studies on how to resolve the factors that affecting the sensor response (such as humidity, interferences, temperature, etc.) are necessary.

7. So far, majority of VOC sensors make use of metal-oxide nanostructures, thereby implementation of devices with emerging halide perovskite nanomaterials can be much anticipated in VOCs detection.

8. Many reports describe the dopant and composited materials tuned sensory responses towards specific VOCs, but there is no-valid information regarding the efficacy and the role of such dopants or composited materials on the VOCs sensing, which requires clarification.

9. Enormous amount of reports are available for acetone and alcohols chemiresistor sensors, thus upcoming researchers must focus towards commercialization rather than simply developing the new materials.

10. Due to the high toxic effect of aldehydes and amines over the eco-systems, chemiresistor sensors coupled with eco-friendly instrumental set up is requires much focus.

11. Mainstream of BTEX assays by distinct nanostructures are still need to be tuned towards specific target due to their ineffectiveness towards mixed analytes. 
12. Detection of other toxic VOCs (such as carbon tetrachloride $\left(\mathrm{CCl}_{4}\right)$, chloroform $\left(\mathrm{CHCl}_{3}\right)$, phosgene, etc.) needs much attention in future.

13. Standardized procedure is become mandatory to attain the specific nanostructures and its VOC sensing performance.

14. Development of stable and commercial devices in the determination of VOCs is still in demand, therefore, much attention is required for commercialization.

15. More research is necessary to justify the exact production cost of reliable devices in VOCs detection with social importance.

Though mechanistic aspects of VOCs detection have been clarified by numerous reports; however, theoretical and in-depth discussions regarding charge/electron transport in semiconducting properties are yet to be improved. As an essential research in health care innovations, many scientists are currently trying to develop and commercialize costeffective devices towards specific VOC targets, which may improve the safety of the healthcare and food products in future.

Author Contributions: M.S. and K.W.S. wrote and proofread the manuscript. All authors have read and agreed to the published version of the manuscript.

Funding: This research was funded by the Ministry of Science and Technology of Taiwan under the contract. MOST 109-2811-M-009-520-MY3 and MOST 109-2112-M-009-013.

Institutional Review Board Statement: Not applicable.

Informed Consent Statement: Not applicable.

Data Availability Statement: No new data were created or analyzed in this study. Data sharing is not applicable to this article.

Conflicts of Interest: The authors declare no conflict of interest.

\section{References}

1. Broza, Y.Y.; Vishinkin, R.; Barash, O.; Nakhleh, M.K.; Haick, H. Synergy between nanomaterials and volatile organic compounds for non-invasive medical evaluation. Chem. Soc. Rev. 2018, 47, 4781-4859. [CrossRef]

2. Broza, Y.Y.; Haick, H. Nanomaterial-based sensors for detection of disease by volatile organic compounds. Nanomedicine 2013, 8, 785-806. [CrossRef] [PubMed]

3. Konvalina, G.; Haick, H. Sensors for Breath Testing: From Nanomaterials to Comprehensive Disease Detection. Acc. Chem. Res. 2014, 47, 66-76. [CrossRef] [PubMed]

4. Xia, H.-C.; Xu, X.-H.; Song, Q.-H. Fluorescent Chemosensor for Selective Detection of Phosgene in Solutions and in Gas Phase. ACS Sens. 2017, 2, 178-182. [CrossRef] [PubMed]

5. Maiti, K.; Ghosh, D.; Maiti, R.; Vyas, V.; Datta, P.; Mandal, D.; Maiti, D.K. Ratiometric chemodosimeter: An organic-nanofiber platform for sensing lethal phosgene gas. J. Mater. Chem. A 2019, 7, 1756-1767. [CrossRef]

6. Mirzaei, A.; Leonardi, S.G.; Neri, G. Detection of hazardous volatile organic compounds (VOCs) by metal oxide nanostructuresbased gas sensors: A review. Ceram. Int. 2016, 42, 15119-15141. [CrossRef]

7. Spinelle, L.; Gerboles, M.; Kok, G.; Persijn, S.; Sauerwald, T. Review of Portable and Low-Cost Sensors for the Ambient Air Monitoring of Benzene and Other Volatile Organic Compounds. Sensors 2017, 17, 1520. [CrossRef] [PubMed]

8. Jalal, A.H.; Alam, F.; Roychoudhury, S.; Umasankar, Y.; Pala, N.; Bhansali, S. Prospects and Challenges of Volatile Organic Compound Sensors in Human Healthcare. ACS Sens. 2018, 3, 1246-1263. [CrossRef]

9. Zhang, D.; Yang, Z.; Yu, S.; Mi, Q.; Pan, Q. Diversiform metal oxide-based hybrid nanostructures for gas sensing with versatile prospects. Coord. Chem. Rev. 2020, 413, 213272. [CrossRef]

10. Swain, S.K.; Barik, S.; Das, R. Nanomaterials as Sensor for Hazardous Gas Detection. In Handbook of Ecomaterials; Martínez, L.M.T., Kharissova, O.V., Kharisov, B.I., Eds.; Springer International Publishing: Cham, Switzerland, 2018; pp. 1-20.

11. Francioso, L.; De Pascali, C.; Creti, P.; Radogna, A.V.; Capone, S.; Taurino, A.; Epifani, M.; Baldacchini, C.; Bizzarri, A.R.; Siciliano, P.A. Nanogap Sensors Decorated with $\mathrm{SnO}_{2}$ Nanoparticles Enable Low-Temperature Detection of Volatile Organic Compounds. ACS Appl. Nano Mater. 2020, 3, 3337-3346. [CrossRef]

12. Maslik, J.; Kuritka, I.; Urbanek, P.; Krcmar, P.; Suly, P.; Masar, M.; Machovsky, M. Water-Based Indium Tin Oxide Nanoparticle Ink for Printed Toluene Vapours Sensor Operating at Room Temperature. Sensors 2018, 18, 3246. [CrossRef]

13. Shellaiah, M.; Sun, K.W. Review on Sensing Applications of Perovskite Nanomaterials. Chemosensors 2020, 8, 55. [CrossRef]

14. Amiri, V.; Roshan, H.; Mirzaei, A.; Neri, G.; Ayesh, A.I. Nanostructured Metal Oxide-Based Acetone Gas Sensors: A Review. Sensors 2020, 20, 3096. [CrossRef] [PubMed] 
15. Prakash Sharma, V.; Sharma, U.; Chattopadhyay, M.; Shukla, V.N. Advance Applications of Nanomaterials: A Review. Mater. Today Proc. 2018, 5, 6376-6380. [CrossRef]

16. Nanomaterials definition matters. Nat. Nanotechnol. 2019, 14, 193. [CrossRef] [PubMed]

17. Kolahalam, L.A.; Kasi Viswanath, I.V.; Diwakar, B.S.; Govindh, B.; Reddy, V.; Murthy, Y.L.N. Review on nanomaterials: Synthesis and applications. Mater. Today Proc. 2019, 18, 2182-2190. [CrossRef]

18. Shellaiah, M.; Sun, K.W. Review on Nanomaterial-Based Melamine Detection. Chemosensors 2019, 7, 9. [CrossRef]

19. Pirzada, M.; Altintas, Z. Nanomaterials for Healthcare Biosensing Applications. Sensors 2019, 19, 5311. [CrossRef]

20. Shellaiah, M.; Chen, Y.-C.; Simon, T.; Li, L.-C.; Sun, K.W.; Ko, F.-H. Effect of Metal Ions on Hybrid Graphite-Diamond Nanowire Growth: Conductivity Measurements from a Single Nanowire Device. Nanomaterials 2019, 9, 415. [CrossRef]

21. Sun, H.; Deng, J.; Qiu, L.; Fang, X.; Peng, H. Recent progress in solar cells based on one-dimensional nanomaterials. Energy Environ. Sci. 2015, 8, 1139-1159. [CrossRef]

22. Rowland, C.E.; Brown, C.W.; Delehanty, J.B.; Medintz, I.L. Nanomaterial-based sensors for the detection of biological threat agents. Mater. Today 2016, 19, 464-477. [CrossRef] [PubMed]

23. Li, B.; Zhou, Q.; Peng, S.; Liao, Y. Recent Advances of $\mathrm{SnO}_{2}$-Based Sensors for Detecting Volatile Organic Compounds. Front. Chem. 2020, 8, 321. [CrossRef] [PubMed]

24. Abdel-Karim, R.; Reda, Y.; Abdel-Fattah, A. Review-Nanostructured Materials-Based Nanosensors. J. Electrochem. Soc. 2020, 167, 037554. [CrossRef]

25. Wang, J.; Zhou, Q.; Peng, S.; Xu, L.; Zeng, W. Volatile Organic Compounds Gas Sensors Based on Molybdenum Oxides: A Mini Review. Front. Chem. 2020, 8, 339. [CrossRef] [PubMed]

26. Malik, R.; Tomer, V.K.; Mishra, Y.K.; Lin, L. Functional gas sensing nanomaterials: A panoramic view. Appl. Phys. Rev. 2020, 7, 021301. [CrossRef]

27. Camilli, L.; Passacantando, M. Advances on Sensors Based on Carbon Nanotubes. Chemosensors 2018, 6, 62. [CrossRef]

28. Schroeder, V.; Savagatrup, S.; He, M.; Lin, S.; Swager, T.M. Carbon Nanotube Chemical Sensors. Chem. Rev. 2019, 119, 599-663. [CrossRef]

29. Zhou, X.; Xue, Z.; Chen, X.; Huang, C.; Bai, W.; Lu, Z.; Wang, T. Nanomaterial-based gas sensors used for breath diagnosis. J. Mater. Chem. B 2020, 8, 3231-3248. [CrossRef]

30. Li, X.; Sun, L.; Sui, H.; He, L.; Yuan, W.; Han, Z. A Novel Polymeric Adsorbent Embedded with Phase Change Materials (PCMs) Microcapsules: Synthesis and Application. Nanomaterials 2019, 9, 736. [CrossRef]

31. Bearzotti, A.; Macagnano, A.; Papa, P.; Venditti, I.; Zampetti, E. A study of a QCM sensor based on pentacene for the detection of BTX vapors in air. Sens. Actuators B 2017, 240, 1160-1164. [CrossRef]

32. Wang, B.; Sonar, P.; Manzhos, S.; Haick, H. Diketopyrrolopyrrole copolymers based chemical sensors for the detection and discrimination of volatile organic compounds. Sens. Actuators B 2017, 251, 49-56. [CrossRef]

33. Wang, Y.; Tao, H.; Yu, D.; Chang, C. Performance Assessment of Ordered Porous Electrospun Honeycomb Fibers for the Removal of Atmospheric Polar Volatile Organic Compounds. Nanomaterials 2018, 8, 350. [CrossRef] [PubMed]

34. Pantalei, S.; Zampetti, E.; Macagnano, A.; Bearzotti, A.; Venditti, I.; Russo, M.V. Enhanced Sensory Properties of a Multichannel Quartz Crystal Microbalance Coated with Polymeric Nanobeads. Sensors 2007, 7, 2920-2928. [CrossRef] [PubMed]

35. Avossa, J.; Paolesse, R.; Di Natale, C.; Zampetti, E.; Bertoni, G.; De Cesare, F.; Scarascia-Mugnozza, G.; Macagnano, A. Electrospinning of Polystyrene/Polyhydroxybutyrate Nanofibers Doped with Porphyrin and Graphene for Chemiresistor Gas Sensors. Nanomaterials 2019, 9, 280. [CrossRef]

36. Walekar, L.; Dutta, T.; Kumar, P.; Ok, Y.S.; Pawar, S.; Deep, A.; Kim, K.-H. Functionalized fluorescent nanomaterials for sensing pollutants in the environment: A critical review. TrAC Trends Anal. Chem. 2017, 97, 458-467. [CrossRef]

37. Andre, R.S.; Sanfelice, R.C.; Pavinatto, A.; Mattoso, L.H.C.; Correa, D.S. Hybrid nanomaterials designed for volatile organic compounds sensors: A review. Mater. Des. 2018, 156, 154-166. [CrossRef]

38. Zhang, C.; Wang, J.; Hu, R.; Qiao, Q.; Li, X. Synthesis and gas sensing properties of porous hierarchical $\mathrm{SnO}_{2}$ by grapefruit exocarp biotemplate. Sens. Actuators B 2016, 222, 1134-1143. [CrossRef]

39. Li, J.; Tang, P.; Zhang, J.; Feng, Y.; Luo, R.; Chen, A.; Li, D. Facile Synthesis and Acetone Sensing Performance of Hierarchical SnO2 Hollow Microspheres with Controllable Size and Shell Thickness. Ind. Eng. Chem. Res. 2016, 55, 3588-3595. [CrossRef]

40. Zhao, R.; Wang, Z.; Zou, T.; Wang, Z.; Xing, X.; Yang, Y.; Wang, Y. ‘Green' prepare $\mathrm{SnO}_{2}$ nanofibers by shaddock peels: Application for detection of volatile organic compound gases. J. Mater. Sci. Mater. Electron. 2019, 30, 3032-3044. [CrossRef]

41. Abdelghani, R.; Shokry Hassan, H.; Morsi, I.; Kashyout, A.B. Nano-architecture of highly sensitive $\mathrm{SnO}_{2}$-based gas sensors for acetone and ammonia using molecular imprinting technique. Sens. Actuators B 2019, 297, 126668. [CrossRef]

42. Li, R.; Chen, S.; Lou, Z.; Li, L.; Huang, T.; Song, Y.; Chen, D.; Shen, G. Fabrication of porous $\mathrm{SnO}_{2}$ nanowires gas sensors with enhanced sensitivity. Sens. Actuators B 2017, 252, 79-85. [CrossRef]

43. Bing, Y.; Liu, C.; Qiao, L.; Zeng, Y.; Yu, S.; Liang, Z.; Liu, J.; Luo, J.; Zheng, W. Multistep synthesis of non-spherical SnO ${ }_{2} @ S n \mathrm{~S}_{2}$ yolk-shell cuboctahedra with nanoparticle-assembled porous structure for toluene detection. Sens. Actuators B 2016, 231, 365-375. [CrossRef]

44. Torrinha, Á.; Oliveira, T.M.B.F.; Ribeiro, F.W.P.; Correia, A.N.; Lima-Neto, P.; Morais, S. Application of Nanostructured CarbonBased Electrochemical (Bio)Sensors for Screening of Emerging Pharmaceutical Pollutants in Waters and Aquatic Species: A Review. Nanomaterials 2020, 10, 1268. [CrossRef] [PubMed] 
45. Waheed, A.; Mansha, M.; Ullah, N. Nanomaterials-based electrochemical detection of heavy metals in water: Current status, challenges and future direction. TrAC Trends Anal. Chem. 2018, 105, 37-51. [CrossRef]

46. Jian, Y.; Hu, W.; Zhao, Z.; Cheng, P.; Haick, H.; Yao, M.; Wu, W. Gas Sensors Based on Chemi-Resistive Hybrid Functional Nanomaterials. Nano-Micro Lett. 2020, 12, 71. [CrossRef]

47. Ferrero, F.J.; Valledor, M.; Campo, J.C.; López, A.; Llano-Suárez, P.; Fernández-Arguelles, M.T.; Costa-Fernández, J.M.; Soldado, A. Portable Instrument for Monitoring Environmental Toxins Using Immobilized Quantum Dots as the Sensing Material. Appl. Sci. 2020, 10, 3246. [CrossRef]

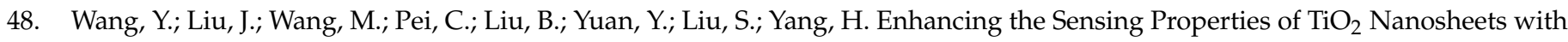
Exposed $\{001\}$ Facets by a Hydrogenation and Sensing Mechanism. Inorg. Chem. 2017, 56, 1504-1510. [CrossRef]

49. Zhou, T.; Sang, Y.; Wang, X.; Wu, C.; Zeng, D.; Xie, C. Pore size dependent gas-sensing selectivity based on ZnO@ZIF nanorod arrays. Sens. Actuators B 2018, 258, 1099-1106. [CrossRef]

50. Dey, S.; Nag, S.; Santra, S.; Ray, S.K.; Guha, P.K. Voltage-controlled NiO/ZnO p-n heterojunction diode: A new approach towards selective VOC sensing. Microsyst. Nanoeng. 2020, 6, 35. [CrossRef]

51. Yeung, H.H.M.; Yoshikawa, G.; Minami, K.; Shiba, K. Strain-based chemical sensing using metal-organic framework nanoparticles. J. Mater. Chem. A 2020, 8, 18007-18014. [CrossRef]

52. Kim, S.-J.; Choi, S.-J.; Jang, J.-S.; Kim, N.-H.; Hakim, M.; Tuller, H.L.; Kim, I.-D. Mesoporous $\mathrm{WO}_{3} \mathrm{Nan}$ ofibers with ProteinTemplated Nanoscale Catalysts for Detection of Trace Biomarkers in Exhaled Breath. ACS Nano 2016, 10, 5891-5899. [CrossRef] [PubMed]

53. Rodner, M.; Puglisi, D.; Ekeroth, S.; Helmersson, U.; Shtepliuk, I.; Yakimova, R.; Skallberg, A.; Uvdal, K.; Schütze, A.; Eriksson, J. Graphene Decorated with Iron Oxide Nanoparticles for Highly Sensitive Interaction with Volatile Organic Compounds. Sensors 2019, 19, 918. [CrossRef]

54. Chen, W.Y.; Yen, C.-C.; Xue, S.; Wang, H.; Stanciu, L.A. Surface Functionalization of Layered Molybdenum Disulfide for the Selective Detection of Volatile Organic Compounds at Room Temperature. ACS Appl. Mater. Interfaces 2019, 11, 34135-34143. [CrossRef] [PubMed]

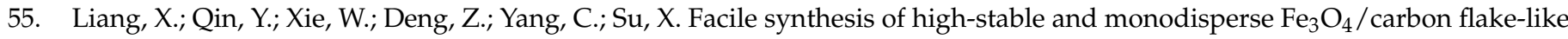
nanocomposites and their excellent gas sensing properties. J. Alloys Compd. 2020, 818, 152898. [CrossRef]

56. Han, X.; Sun, Y.; Feng, Z.; Zhang, G.; Chen, Z.; Zhan, J. Au-deposited porous single-crystalline ZnO nanoplates for gas sensing detection of total volatile organic compounds. RSC Adv. 2016, 6, 37750-37756. [CrossRef]

57. Han, T.-L.; Wan, Y.-T.; Li, J.-J.; Zhang, H.-G.; Liu, J.-H.; Huang, X.-J.; Liu, J.-Y. In situ gold nanoparticle-decorated three-dimensional tin dioxide nanostructures for sensitive and selective gas-sensing detection of volatile organic compounds. J. Mater. Chem. C 2017, 5, 6193-6201. [CrossRef]

58. Postica, V.; Vahl, A.; Santos-Carballal, D.; Dankwort, T.; Kienle, L.; Hoppe, M.; Cadi-Essadek, A.; de Leeuw, N.H.; Terasa, M.-I.; Adelung, R.; et al. Tuning ZnO Sensors Reactivity toward Volatile Organic Compounds via Ag Doping and Nanoparticle Functionalization. ACS Appl. Mater. Interfaces 2019, 11, 31452-31466. [CrossRef]

59. Navale, S.T.; Yang, Z.B.; Liu, C.; Cao, P.J.; Patil, V.B.; Ramgir, N.S.; Mane, R.S.; Stadler, F.J. Enhanced acetone sensing properties of titanium dioxide nanoparticles with a sub-ppm detection limit. Sens. Actuators B 2018, 255, 1701-1710. [CrossRef]

60. Liang, S.; Li, J.; Wang, F.; Qin, J.; Lai, X.; Jiang, X. Highly sensitive acetone gas sensor based on ultrafine $\alpha-\mathrm{Fe}_{2} \mathrm{O}_{3}$ nanoparticles. Sens. Actuators B 2017, 238, 923-927. [CrossRef]

61. Zhou, X.; Wang, J.; Wang, Z.; Bian, Y.; Wang, Y.; Han, N.; Chen, Y. Transilient Response to Acetone Gas Using the Interlocking $\mathrm{p}+\mathrm{n}$ Field-Effect Transistor Circuit. Sensors 2018, 18, 1914. [CrossRef]

62. Koo, A.; Yoo, R.; Woo, S.P.; Lee, H.-S.; Lee, W. Enhanced acetone-sensing properties of pt-decorated al-doped ZnO nanoparticles. Sens. Actuators B 2019, 280, 109-119. [CrossRef]

63. Yoo, R.; Park, Y.; Jung, H.; Rim, H.J.; Cho, S.; Lee, H.-S.; Lee, W. Acetone-sensing properties of doped ZnO nanoparticles for breath-analyzer applications. J. Alloys Compd. 2019, 803, 135-144. [CrossRef]

64. Yang, W.; Shen, H.; Min, H.; Ge, J. Enhanced acetone sensing performance in black $\mathrm{TiO}_{2}$ by Ag modification. J. Mater. Sci. 2020, 55, 10399-10411. [CrossRef]

65. Liu, H.; Li, C.; Zhang, X.; Zheng, K.; Xie, R.; Huang, H.; Peng, T.; Jia, R.; Huo, J. A novel and highly responsive acetone sensor based on $\mathrm{La}_{1-\mathrm{x}} \mathrm{Y}_{\mathrm{x}} \mathrm{MnO}_{3+\delta}$ nanoparticles. Mater. Lett. 2019, 257, 126725. [CrossRef]

66. Peng, S.; Ma, M.; Yang, W.; Wang, Z.; Wang, Z.; Bi, J.; Wu, J. Acetone sensing with parts-per-billion limit of detection using a $\mathrm{BiFeO}_{3}$-based solid solution sensor at the morphotropic phase boundary. Sens. Actuators B 2020, 313, 128060. [CrossRef]

67. Zhang, H.; Qin, H.; Zhang, P.; Hu, J. High Sensing Properties of 3 wt \% Pd-Doped $\mathrm{SmFe}_{1-\mathrm{x}} \mathrm{Mg}_{\mathrm{x}} \mathrm{O}_{3}$ Nanocrystalline Powders to Acetone Vapor with Ultralow Concentrations under Light Illumination. ACS Appl. Mater. Interfaces 2018, 10, 15558-15564. [CrossRef]

68. Lu, J.; Xu, C.; Cheng, L.; Jia, N.; Huang, J.; Li, C. Acetone sensor based on $\mathrm{WO}_{3}$ nanocrystallines with oxygen defects for low concentration detection. Mater. Sci. Semicond. Proc. 2019, 101, 214-222. [CrossRef]

69. Epifani, M.; Kaciulis, S.; Mezzi, A.; Zhang, T.; Arbiol, J.; Siciliano, P.; Landström, A.; Concina, I.; Moumen, A.; Comini, E.; et al. Rhodium as efficient additive for boosting acetone sensing by $\mathrm{TiO}_{2}$ nanocrystals. Beyond the classical view of noble metal additives. Sens. Actuators B 2020, 319, 128338. [CrossRef] 
70. Kim, H.; Cai, Z.; Chang, S.-P.; Park, S. Improved sub-ppm acetone sensing properties of $\mathrm{SnO}_{2}$ nanowire-based sensor by attachment of $\mathrm{Co}_{3} \mathrm{O}_{4}$ nanoparticles. J. Mater. Res. Technol. 2020, 9, 1129-1136. [CrossRef]

71. Singh, M.; Kaur, N.; Drera, G.; Casotto, A.; Sangaletti, L.; Comini, E. SAM Functionalized ZnO Nanowires for Selective Acetone Detection: Optimized Surface Specific Interaction Using APTMS and GLYMO Monolayers. Adv. Funct. Mater. 2020, 30, 2003217. [CrossRef]

72. Xue, X.-T.; Zhu, L.-Y.; Yuan, K.-P.; Zeng, C.; Li, X.-X.; Ma, H.-P.; Lu, H.-L.; Zhang, D.W. ZnO branched p-CuxO@n-ZnO heterojunction nanowires for improving acetone gas sensing performance. Sens. Actuators B 2020, 324, 128729. [CrossRef]

73. Zhang, G.H.; Deng, X.Y.; Wang, P.Y.; Wang, X.L.; Chen, Y.; Ma, H.L.; Gengzang, D.J. Morphology controlled syntheses of Cr doped $\mathrm{ZnO}$ single-crystal nanorods for acetone gas sensor. Mater. Lett. 2016, 165, 83-86. [CrossRef]

74. Giberti, A.; Gaiardo, A.; Fabbri, B.; Gherardi, S.; Guidi, V.; Malagù, C.; Bellutti, P.; Zonta, G.; Casotti, D.; Cruciani, G. Tin(IV) sulfide nanorods as a new gas sensing material. Sens. Actuators B 2016, 223, 827-833. [CrossRef]

75. Huang, J.; Zhou, J.; Liu, Z.; Li, X.; Geng, Y.; Tian, X.; Du, Y.; Qian, Z. Enhanced acetone-sensing properties to ppb detection level using Au/Pd-doped ZnO nanorod. Sens. Actuators B 2020, 310, 127129. [CrossRef]

76. Wang, Z.; Zhang, K.; Fei, T.; Gu, F.; Han, D. $\alpha-\mathrm{Fe}_{2} \mathrm{O}_{3} / \mathrm{NiO}$ heterojunction nanorods with enhanced gas sensing performance for acetone. Sens. Actuators B 2020, 318, 128191. [CrossRef]

77. Al-Hadeethi, Y.; Umar, A.; Ibrahim, A.A.; Al-Heniti, S.H.; Kumar, R.; Baskoutas, S.; Raffah, B.M. Synthesis, characterization and acetone gas sensing applications of Ag-doped $\mathrm{ZnO}$ nanoneedles. Ceram. Int. 2017, 43, 6765-6770. [CrossRef]

78. Gao, F.; Qin, G.; Li, Y.; Jiang, Q.; Luo, L.; Zhao, K.; Liu, Y.; Zhao, H. One-pot synthesis of La-doped SnO 2 layered nanoarrays with an enhanced gas-sensing performance toward acetone. RSC Adv. 2016, 6, 10298-10310. [CrossRef]

79. Gong, H.; Zhao, C.; Niu, G.; Zhang, W.; Wang, F. Construction of 1D/2D $\alpha-\mathrm{Fe}_{2} \mathrm{O}_{3} / \mathrm{SnO}_{2}$ Hybrid Nanoarrays for Sub-ppm Acetone Detection. Research 2020, 2020, 2196063. [CrossRef]

80. Abdul Haroon Rashid, S.S.A.; Sabri, Y.M.; Kandjani, A.E.; Harrison, C.J.; Canjeevaram Balasubramanyam, R.K.; Della Gaspera, E.; Field, M.R.; Bhargava, S.K.; Tricoli, A.; Wlodarski, W.; et al. Zinc Titanate Nanoarrays with Superior Optoelectrochemical Properties for Chemical Sensing. ACS Appl. Mater. Interfaces 2019, 11, 29255-29267. [CrossRef]

81. Xu, X.; Chen, Y.; Zhang, G.; Ma, S.; Lu, Y.; Bian, H.; Chen, Q. Highly sensitive VOCs-acetone sensor based on Ag-decorated SnO 2 hollow nanofibers. J. Alloys Compd. 2017, 703, 572-579. [CrossRef]

82. Ma, L.; Ma, S.Y.; Shen, X.F.; Wang, T.T.; Jiang, X.H.; Chen, Q.; Qiang, Z.; Yang, H.M.; Chen, H. PrFeO 3 hollow nanofibers as a highly efficient gas sensor for acetone detection. Sens. Actuators B 2018, 255, 2546-2554. [CrossRef]

83. Guo, L.; Chen, F.; Xie, N.; Kou, X.; Wang, C.; Sun, Y.; Liu, F.; Liang, X.; Gao, Y.; Yan, X.; et al. Ultra-sensitive sensing platform based on Pt-ZnO-In2O3 nanofibers for detection of acetone. Sens. Actuators B 2018, 272, 185-194. [CrossRef]

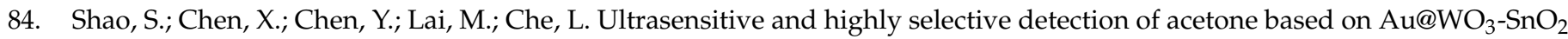
corrugated nanofibers. Appl. Surf. Sci. 2019, 473, 902-911. [CrossRef]

85. Chen, Q.; Wang, Y.; Wang, M.; Ma, S.; Wang, P.; Zhang, G.; Chen, W.; Jiao, H.; Liu, L.; Xu, X. Enhanced acetone sensor based on Au functionalized In-doped $\mathrm{ZnSnO}_{3}$ nanofibers synthesized by electrospinning method. J. Colloid Interface Sci. 2019, 543, 285-299. [CrossRef] [PubMed]

86. Du, H.; Yang, W.; Yi, W.; Sun, Y.; Yu, N.; Wang, J. Oxygen-Plasma-Assisted Enhanced Acetone-Sensing Properties of ZnO Nanofibers by Electrospinning. ACS Appl. Mater. Interfaces 2020, 12, 23084-23093. [CrossRef]

87. Kou, X.; Meng, F.; Chen, K.; Wang, T.; Sun, P.; Liu, F.; Yan, X.; Sun, Y.; Liu, F.; Shimanoe, K.; et al. High-performance acetone gas sensor based on Ru-doped $\mathrm{SnO}_{2}$ nanofibers. Sens. Actuators B 2020, 320, 128292. [CrossRef]

88. Jang, J.-S.; Yu, S.; Choi, S.-J.; Kim, S.-J.; Koo, W.-T.; Kim, I.-D. Metal Chelation Assisted In Situ Migration and Functionalization of Catalysts on Peapod-Like Hollow $\mathrm{SnO}_{2}$ toward a Superior Chemical Sensor. Small 2016, 12, 5989-5997. [CrossRef] [PubMed]

89. Koo, W.-T.; Jang, J.-S.; Choi, S.-J.; Cho, H.-J.; Kim, I.-D. Metal-Organic Framework Templated Catalysts: Dual Sensitization of PdO-ZnO Composite on Hollow $\mathrm{SnO}_{2}$ Nanotubes for Selective Acetone Sensors. ACS Appl. Mater. Interfaces 2017, 9, 18069-18077. [CrossRef]

90. Dai, M.; Zhao, L.; Gao, H.; Sun, P.; Liu, F.; Zhang, S.; Shimanoe, K.; Yamazoe, N.; Lu, G. Hierarchical Assembly of $\alpha$-Fe ${ }_{2} \mathrm{O}_{3}$ Nanorods on Multiwall Carbon Nanotubes as a High-Performance Sensing Material for Gas Sensors. ACS Appl. Mater. Interfaces 2017, 9, 8919-8928. [CrossRef]

91. Zhang, R.; Zhang, M.; Zhou, T.; Zhang, T. Robust cobalt perforated with multi-walled carbon nanotubes as an effective sensing material for acetone detection. Inorg. Chem. Front. 2018, 5, 2563-2570. [CrossRef]

92. Zhao, C.; Lan, W.; Gong, H.; Bai, J.; Ramachandran, R.; Liu, S.; Wang, F. Highly sensitive acetone-sensing properties of Pt-decorated $\mathrm{CuFe}_{2} \mathrm{O}_{4}$ nanotubes prepared by electrospinning. Ceram. Int. 2018, 44, 2856-2863. [CrossRef]

93. Zhang, J.; Zhang, L.; Leng, D.; Ma, F.; Zhang, Z.; Zhang, Y.; Wang, W.; Liang, Q.; Gao, J.; Lu, H. Nanoscale Pd catalysts decorated $\mathrm{WO}_{3}-\mathrm{SnO}_{2}$ heterojunction nanotubes for highly sensitive and selective acetone sensing. Sens. Actuators B 2020, $306,127575$. [CrossRef]

94. Zhang, Y.; Jia, C.; Kong, Q.; Fan, N.; Chen, G.; Guan, H.; Dong, C. ZnO-Decorated In/Ga Oxide Nanotubes Derived from Bimetallic In/Ga MOFs for Fast Acetone Detection with High Sensitivity and Selectivity. ACS Appl. Mater. Interfaces 2020, 12, 26161-26169. [CrossRef] [PubMed]

95. Li, X.; Liu, Y.; Li, S.; Huang, J.; Wu, Y.; Yu, D. The Sensing Properties of Single Y-Doped $\mathrm{SnO}_{2}$ Nanobelt Device to Acetone. Nanoscale Res. Lett. 2016, 11, 470. [CrossRef] 
96. Chen, W.; Qin, Z.; Liu, Y.; Zhang, Y.; Li, Y.; Shen, S.; Wang, Z.M.; Song, H.-Z. Promotion on Acetone Sensing of Single SnO 2 Nanobelt by Eu Doping. Nanoscale Res. Lett. 2017, 12, 405. [CrossRef]

97. Zhou, T.; Zhang, T.; Deng, J.; Zhang, R.; Lou, Z.; Wang, L. P-type $\mathrm{Co}_{3} \mathrm{O}_{4}$ nanomaterials-based gas sensor: Preparation and acetone sensing performance. Sens. Actuators B 2017, 242, 369-377. [CrossRef]

98. Mishra, R.K.; Murali, G.; Kim, T.-H.; Kim, J.H.; Lim, Y.J.; Kim, B.-S.; Sahay, P.P.; Lee, S.H. Nanocube $\mathrm{In}_{2} \mathrm{O}_{3} @ R G O$ heterostructure based gas sensor for acetone and formaldehyde detection. RSC Adv. 2017, 7, 38714-38724. [CrossRef]

99. Yin, Y.; Li, F.; Zhang, N.; Ruan, S.; Zhang, H.; Chen, Y. Improved gas sensing properties of silver-functionalized $\mathrm{ZnSnO}_{3}$ hollow nanocubes. Inorg. Chem. Front. 2018, 5, 2123-2131. [CrossRef]

100. Lee, J.E.; Lim, C.K.; Park, H.J.; Song, H.; Choi, S.-Y.; Lee, D.-S. ZnO-CuO Core-Hollow Cube Nanostructures for Highly Sensitive Acetone Gas Sensors at the ppb Level. ACS Appl. Mater. Interfaces 2020, 12, 35688-35697. [CrossRef]

101. Wang, X.-F.; Ma, W.; Jiang, F.; Cao, E.-S.; Sun, K.-M.; Cheng, L.; Song, X.-Z. Prussian Blue analogue derived porous NiFe ${ }_{2} \mathrm{O}_{4}$ nanocubes for low-concentration acetone sensing at low working temperature. Chem. Eng. J. 2018, 338, 504-512. [CrossRef]

102. Zhang, N.; Li, H.; Xu, Z.; Yuan, R.; Xu, Y.; Cui, Y. Enhanced Acetone Sensing Property of a Sacrificial Template Based on Cubic-Like MOF-5 Doped by Ni Nanoparticles. Nanomaterials 2020, 10, 386. [CrossRef]

103. Ma, X.; Zhou, X.; Gong, Y.; Han, N.; Liu, H.; Chen, Y. MOF-derived hierarchical $\mathrm{ZnO} / \mathrm{ZnFe}_{2} \mathrm{O}_{4}$ hollow cubes for enhanced acetone gas-sensing performance. RSC Adv. 2017, 7, 34609-34617. [CrossRef]

104. Koo, W.-T.; Yu, S.; Choi, S.-J.; Jang, J.-S.; Cheong, J.Y.; Kim, I.-D. Nanoscale PdO Catalyst Functionalized Co3O4 Hollow Nanocages Using MOF Templates for Selective Detection of Acetone Molecules in Exhaled Breath. ACS Appl. Mater. Interfaces 2017, 9, 8201-8210. [CrossRef] [PubMed]

105. Wang, X.; Zhang, S.; Shao, M.; Huang, J.; Deng, X.; Hou, P.; Xu, X. Fabrication of $\mathrm{ZnO} / \mathrm{ZnFe}_{2} \mathrm{O}_{4}$ hollow nanocages through metal organic frameworks route with enhanced gas sensing properties. Sens. Actuators B 2017, 251, 27-33. [CrossRef]

106. Zhou, T.; Liu, X.; Zhang, R.; Wang, Y.; Zhang, T. NiO/ $\mathrm{NiCO}_{2} \mathrm{O}_{4}$ Truncated Nanocages with PdO Catalyst Functionalization as Sensing Layers for Acetone Detection. ACS Appl. Mater. Interfaces 2018, 10, 37242-37250. [CrossRef] [PubMed]

107. Wang, G.; Fu, Z.; Wang, T.; Lei, W.; Sun, P.; Sui, Y.; Zou, B. A rational design of hollow nanocages $\mathrm{Ag} @ \mathrm{CuO}^{-\mathrm{TiO}} 2$ for enhanced acetone sensing performance. Sens. Actuators $B$ 2019, 295, 70-78. [CrossRef]

108. Zhang, Z.; Zhu, L.; Wen, Z.; Ye, Z. Controllable synthesis of $\mathrm{Co}_{3} \mathrm{O}_{4}$ crossed nanosheet arrays toward an acetone gas sensor. Sens. Actuators B 2017, 238, 1052-1059. [CrossRef]

109. Li, S.-M.; Zhang, L.-X.; Zhu, M.-Y.; Ji, G.-J.; Zhao, L.-X.; Yin, J.; Bie, L.-J. Acetone sensing of ZnO nanosheets synthesized using room-temperature precipitation. Sens. Actuators B 2017, 249, 611-623. [CrossRef]

110. Liu, J.; Wang, Y.; Wang, L.; Tian, H.; Zeng, Y. Controllable assembly of sandwich-structured $\mathrm{SnO}_{2} / \mathrm{Fe}_{2} \mathrm{O}_{3}$ multilayer nanosheets for high sensitive acetone detection. Mater. Lett. 2018, 221, 57-61. [CrossRef]

111. Dey, S.; Santra, S.; Guha, P.K.; Ray, S.K. Liquid Exfoliated NiO Nanosheets for Trace Level Detection of Acetone Vapors. IEEE Trans. Electron Devices 2019, 66, 3568-3572. [CrossRef]

112. Feng, Z.; Zhang, L.; Chen, W.; Peng, Z.; Li, Y. A strategy for supportless sensors: Fluorine doped $\mathrm{TiO}_{2}$ nanosheets directly grown onto Ti foam enabling highly sensitive detection toward acetone. Sens. Actuators B 2020, 322, 128633. [CrossRef]

113. Kim, S.-H.; Shim, G.-I.; Choi, S.-Y. Fabrication of Nb-doped ZnO nanowall structure by RF magnetron sputter for enhanced gas-sensing properties. J. Alloys Compd. 2017, 698, 77-86. [CrossRef]

114. Hien, V.X.; Minh, N.H.; Son, D.T.; Nghi, N.T.; Phuoc, L.H.; Khoa, C.T.; Vuong, D.D.; Chien, N.D.; Heo, Y.-W. Acetone sensing properties of $\mathrm{CuO}$ nanowalls synthesized via oxidation of $\mathrm{Cu}$ foil in aqueous $\mathrm{NH}_{4} \mathrm{OH}$. Vacuum 2018, 150, 129-135. [CrossRef]

115. Urso, M.; Leonardi, S.G.; Neri, G.; Petralia, S.; Conoci, S.; Priolo, F.; Mirabella, S. Acetone sensing and modelling by low-cost NiO nanowalls. Mater. Lett. 2020, 262, 127043. [CrossRef]

116. Choi, H.; Kwon, S.H.; Kang, H.; Kim, J.H.; Choi, W. Zinc-oxide-deposited Carbon Nanowalls for Acetone Sensing. Thin Solid Films 2020, 700, 137887. [CrossRef]

117. Dwivedi, P.; Dhanekar, S.; Das, S. Synthesis of $\alpha$-MoO3nano-flakes by dry oxidation of RF sputtered Mo thin films and their application in gas sensing. Semicond. Sci. Technol. 2016, 31, 115010. [CrossRef]

118. Afsar, M.F.; Rafiq, M.A.; Tok, A.I.Y. Two-dimensional SnS nanoflakes: Synthesis and application to acetone and alcohol sensors. RSC Adv. 2017, 7, 21556-21566. [CrossRef]

119. Cho, S.-Y.; Koh, H.-J.; Yoo, H.-W.; Kim, J.-S.; Jung, H.-T. Tunable Volatile-Organic-Compound Sensor by Using Au Nanoparticle Incorporation on $\mathrm{MoS}_{2}$. ACS Sens. 2017, 2, 183-189. [CrossRef]

120. Zhang, R.; Wang, Y.; Zhang, Z.; Cao, J. Highly Sensitive Acetone Gas Sensor Based on g- $\mathrm{C}_{3} \mathrm{~N}_{4} \mathrm{Decorated} \mathrm{MgFe}_{2} \mathrm{O}_{4} \mathrm{Porous}$ Microspheres Composites. Sensors 2018, 18, 2211. [CrossRef]

121. Qu, F.; Zhang, N.; Zhang, S.; Zhao, R.; Yao, D.; Ruan, S.; Yang, M. Construction of $\mathrm{Co}_{3} \mathrm{O}_{4} / \mathrm{CoWO}_{4}$ core-shell urchin-like microspheres through ion-exchange method for high-performance acetone gas sensing performance. Sens. Actuators B 2020, 309, 127711. [CrossRef]

122. Liu, C.; Zhao, L.; Wang, B.; Sun, P.; Wang, Q.; Gao, Y.; Liang, X.; Zhang, T.; Lu, G. Acetone gas sensor based on NiO/ZnO hollow spheres: Fast response and recovery, and low (ppb) detection limit. J. Colloid Interface Sci. 2017, 495, 207-215. [CrossRef] [PubMed]

123. Zhu, Y.; Wang, H.; Liu, J.; Yin, M.; Yu, L.; Zhou, J.; Liu, Y.; Qiao, F. High-performance gas sensors based on the $\mathrm{WO}_{3}-\mathrm{SnO}_{2}$ nanosphere composites. J. Alloys Compd. 2019, 782, 789-795. [CrossRef] 
124. Jaisutti, R.; Lee, M.; Kim, J.; Choi, S.; Ha, T.-J.; Kim, J.; Kim, H.; Park, S.K.; Kim, Y.-H. Ultrasensitive Room-Temperature Operable Gas Sensors Using p-Type Na:ZnO Nanoflowers for Diabetes Detection. ACS Appl. Mater. Interfaces 2017, 9, 8796-8804. [CrossRef]

125. Chen, F.; Yang, M.; Wang, X.; Song, Y.; Guo, L.; Xie, N.; Kou, X.; Xu, X.; Sun, Y.; Lu, G. Template-free synthesis of cubicrhombohedral- $\mathrm{In}_{2} \mathrm{O}_{3}$ flower for ppb level acetone detection. Sens. Actuators B 2019, 290, 459-466. [CrossRef]

126. Wang, P.; Dong, T.; Jia, C.; Yang, P. Ultraselective acetone-gas sensor based ZnO flowers functionalized by Au nanoparticle loading on certain facet. Sens. Actuators B 2019, 288, 1-11. [CrossRef]

127. Zhang, S.; Wang, C.; Qu, F.; Liu, S.; Lin, C.-T.; Du, S.; Chen, Y.; Meng, F.; Yang, M. ZnO nanoflowers modified with RuO 2 for enhancing acetone sensing performance. Nanotechnology 2019, 31, 115502. [CrossRef]

128. Ma, T.; Zheng, L.; Zhao, Y.; Xu, Y.; Zhang, J.; Liu, X. Highly Porous Double-Shelled Hollow Hematite Nanoparticles for Gas Sensing. ACS Appl. Nano Mater. 2019, 2, 2347-2357. [CrossRef]

129. Xia, J.; Diao, K.; Zheng, Z.; Cui, X. Porous Au/ZnO nanoparticles synthesised through a metal organic framework (MOF) route for enhanced acetone gas-sensing. RSC Adv. 2017, 7, 38444-38451. [CrossRef]

130. Li, L.; Tan, J.; Dun, M.; Huang, X. Porous $\mathrm{ZnFe}_{2} \mathrm{O}_{4}$ nanorods with net-worked nanostructure for highly sensor response and fast response acetone gas sensor. Sens. Actuators B 2017, 248, 85-91. [CrossRef]

131. Chen, Y.; Li, H.; Ma, Q.; Che, Q.; Wang, J.; Wang, G.; Yang, P. Morphology-controlled porous $\alpha-\mathrm{Fe}_{2} \mathrm{O}_{3} / \mathrm{SnO}_{2}$ nanorods with uniform surface heterostructures and their enhanced acetone gas-sensing properties. Mater. Lett. 2018, 211, 212-215. [CrossRef]

132. Xu, Y.; Lou, C.; Zheng, L.; Zheng, W.; Liu, X.; Kumar, M.; Zhang, J. Highly sensitive and selective detection of acetone based on platinum sensitized porous tungsten oxide nanospheres. Sens. Actuators B 2020, 307, 127616. [CrossRef]

133. Chao, J.; Chen, Y.; Xing, S.; Zhang, D.; Shen, W. Facile fabrication of ZnO/C nanoporous fibers and ZnO hollow spheres for high performance gas sensor. Sens. Actuators B 2019, 298, 126927. [CrossRef]

134. Quan, W.; Hu, X.; Min, X.; Qiu, J.; Tian, R.; Ji, P.; Qin, W.; Wang, H.; Pan, T.; Cheng, S.; et al. A Highly Sensitive and Selective ppb-Level Acetone Sensor Based on a Pt-Doped 3D Porous $\mathrm{SnO}_{2}$ Hierarchical Structure. Sensors 2020, 20, 1150. [CrossRef]

135. Zhang, X.; Dong, Z.; Liu, S.; Shi, Y.; Dong, Y.; Feng, W. Maize straw-templated hierarchical porous ZnO:Ni with enhanced acetone gas sensing properties. Sens. Actuators B 2017, 243, 1224-1230. [CrossRef]

136. Li, X.; Lu, D.; Shao, C.; Lu, G.; Li, X.; Liu, Y. Hollow $\mathrm{CuFe}_{2} \mathrm{O}_{4} / \alpha-\mathrm{Fe}_{2} \mathrm{O}_{3}$ composite with ultrathin porous shell for acetone detection at ppb levels. Sens. Actuators $B$ 2018, 258, 436-446. [CrossRef]

137. Zhang, X.; Dong, B.; Liu, W.; Zhou, X.; Liu, M.; Sun, X.; Lv, J.; Zhang, L.; Xu, W.; Bai, X.; et al. Highly sensitive and selective acetone sensor based on three-dimensional ordered $\mathrm{WO}_{3} / \mathrm{Au}$ nanocomposite with enhanced performance. Sens. Actuators B 2020, 320, 128405. [CrossRef]

138. Choi, H.-J.; Choi, S.-J.; Choo, S.; Kim, I.-D.; Lee, H. Hierarchical ZnO Nanowires-loaded Sb-doped SnO 2 -ZnO Micrograting Pattern via Direct Imprinting-assisted Hydrothermal Growth and Its Selective Detection of Acetone Molecules. Sci. Rep. 2016, 6, 18731. [CrossRef]

139. Peng, C.; Guo, J.; Yang, W.; Shi, C.; Liu, M.; Zheng, Y.; Xu, J.; Chen, P.; Huang, T.; Yang, Y. Synthesis of three-dimensional flower-like hierarchical $\mathrm{ZnO}$ nanostructure and its enhanced acetone gas sensing properties. J. Alloys Compd. 2016, 654, 371-378. [CrossRef]

140. Wang, L.; Fu, H.; Jin, Q.; Jin, H.; Haick, H.; Wang, S.; Yu, K.; Deng, S.; Wang, Y. Directly transforming SnS 2 nanosheets to hierarchical $\mathrm{SnO}_{2}$ nanotubes: Towards sensitive and selective sensing of acetone at relatively low operating temperatures. Sens. Actuators B 2019, 292, 148-155. [CrossRef]

141. Xing, R.; Sheng, K.; Xu, L.; Liu, W.; Song, J.; Song, H. Three-dimensional $\mathrm{In}_{2} \mathrm{O}_{3}-\mathrm{CuO}$ inverse opals: Synthesis and improved gas sensing properties towards acetone. RSC Adv. 2016, 6, 57389-57395. [CrossRef]

142. Dankeaw, A.; Poungchan, G.; Panapoy, M.; Ksapabutr, B. In-situ one-step method for fabricating three-dimensional grass-like carbon-doped $\mathrm{ZrO}_{2}$ films for room temperature alcohol and acetone sensors. Sens. Actuators B 2017, 242, 202-214. [CrossRef]

143. Zhang, Y.; Zhou, L.; Liu, Y.; Liu, D.; Liu, F.; Liu, F.; Yan, X.; Liang, X.; Gao, Y.; Lu, G. Gas sensor based on samarium oxide loaded mulberry-shaped tin oxide for highly selective and sub ppm-level acetone detection. J. Colloid Interface Sci. 2018, 531, 74-82. [CrossRef] [PubMed]

144. Zhu, L.; Zeng, W.; Li, Y. A novel cactus-like $\mathrm{WO}_{3}-\mathrm{SnO}_{2}$ nanocomposite and its acetone gas sensing properties. Mater. Lett. 2018, 231, 5-7. [CrossRef]

145. Shen, J.-Y.; Wang, M.-D.; Wang, Y.-F.; Hu, J.-Y.; Zhu, Y.; Zhang, Y.X.; Li, Z.-J.; Yao, H.-C. Iron and carbon codoped WO 3 with hierarchical walnut-like microstructure for highly sensitive and selective acetone sensor. Sens. Actuators B 2018, 256, 27-37. [CrossRef]

146. Ding, Q.; Wang, Y.; Guo, P.; Li, J.; Chen, C.; Wang, T.; Sun, K.; He, D. Cr-Doped Urchin-Like $\mathrm{WO}_{3} \mathrm{Hollow}$ Spheres: The Cooperative Modulation of Crystal Growth and Energy-Band Structure for High-Sensitive Acetone Detection. Sensors 2020, 20, 3473. [CrossRef]

147. Chang, X.; Qiao, X.; Li, K.; Wang, P.; Xiong, Y.; Li, X.; Xia, F.; Xue, Q. UV assisted ppb-level acetone detection based on hollow $\mathrm{ZnO} / \mathrm{MoS}_{2}$ nanosheets core/shell heterostructures at low temperature. Sens. Actuators B 2020, 317, 128208. [CrossRef]

148. Perfecto, T.M.; Zito, C.A.; Volanti, D.P. Room-temperature volatile organic compounds sensing based on $\mathrm{WO}_{3} \cdot 0.33 \mathrm{H}_{2} \mathrm{O}$, hexagonal$\mathrm{WO}_{3}$, and their reduced graphene oxide composites. RSC Adv. 2016, 6, 105171-105179. [CrossRef]

149. Hu, J.; Zou, C.; Su, Y.; Li, M.; Yang, Z.; Ge, M.; Zhang, Y. One-step synthesis of $2 \mathrm{D} \mathrm{C}_{3} \mathrm{~N}_{4}$-tin oxide gas sensors for enhanced acetone vapor detection. Sens. Actuators B 2017, 253, 641-651. [CrossRef] 
150. Tomer, V.K.; Singh, K.; Kaur, H.; Shorie, M.; Sabherwal, P. Rapid acetone detection using indium loaded $\mathrm{WO}_{3} / \mathrm{SnO}_{2}$ nanohybrid sensor. Sens. Actuators B 2017, 253, 703-713. [CrossRef]

151. Zhang, C.; Li, L.; Hou, L.; Chen, W. Fabrication of $\mathrm{Co}_{3} \mathrm{O}_{4}$ nanowires assembled on the surface of hollow carbon spheres for acetone gas sensing. Sens. Actuators B 2019, 291, 130-140. [CrossRef]

152. Cao, E.; Song, G.; Guo, Z.; Zhang, Y.; Hao, W.; Sun, L.; Nie, Z. Acetone sensing characteristics of $\mathrm{Fe}_{2} \mathrm{O}_{3} / \mathrm{In}_{2} \mathrm{O}_{3}$ nanocomposite. Mater. Lett. 2020, 261, 126985. [CrossRef]

153. Dyndal, K.; Zarzycki, A.; Andrysiewicz, W.; Grochala, D.; Marszalek, K.; Rydosz, A. CuO-Ga ${ }_{2} \mathrm{O}_{3}$ Thin Films as a Gas-Sensitive Material for Acetone Detection. Sensors 2020, 20, 3142. [CrossRef]

154. Qu, F.; Yuan, Y.; Yang, M. Programmed Synthesis of $\mathrm{Sn}_{3} \mathrm{~N}_{4}$ Nanoparticles via a Soft Chemistry Approach with Urea: Application for Ethanol Vapor Sensing. Chem. Mater. 2017, 29, 969-974. [CrossRef]

155. Lin, Z.; Li, N.; Chen, Z.; Fu, P. The effect of Ni doping concentration on the gas sensing properties of $\mathrm{Ni}_{\text {doped }} \mathrm{SnO} \mathrm{O}_{2}$. Sens. Actuators B 2017, 239, 501-510. [CrossRef]

156. Zhao, D.; Zhang, X.; Sui, L.; Wang, W.; Zhou, X.; Cheng, X.; Gao, S.; Xu, Y.; Huo, L. C-doped TiO 2 nanoparticles to detect alcohols with different carbon chains and their sensing mechanism analysis. Sens. Actuators B 2020, 312, 127942. [CrossRef]

157. Ma, Z.-H.; Yu, R.-T.; Song, J.-M. Facile synthesis of Pr-doped $\operatorname{In}_{2} \mathrm{O}_{3}$ nanoparticles and their high gas sensing performance for ethanol. Sens. Actuators B 2020, 305, 127377. [CrossRef]

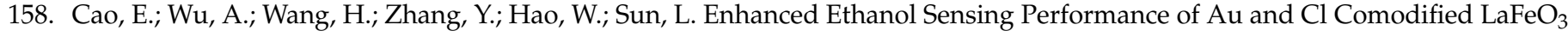
Nanoparticles. ACS Appl. Nano Mater. 2019, 2, 1541-1551. [CrossRef]

159. Cao, K.; Cao, E.; Zhang, Y.; Hao, W.; Sun, L.; Peng, H. The influence of nonstoichiometry on electrical transport and ethanol sensing characteristics for nanocrystalline $\mathrm{LaFe}_{x} \mathrm{O}_{3-\delta}$ sensors. Sens. Actuators B 2016, 230, 592-599. [CrossRef]

160. Cao, E.; Wang, H.; Wang, X.; Yang, Y.; Hao, W.; Sun, L.; Zhang, Y. Enhanced ethanol sensing performance for chlorine doped nanocrystalline $\mathrm{LaFeO}_{3-\delta}$ powders by citric sol-gel method. Sens. Actuators B 2017, 251, 885-893. [CrossRef]

161. Sau, S.; Chakraborty, S.; Das, T.; Pal, M. Ethanol Sensing Properties of Nanocrystalline $\alpha$-MoO3. Front. Mater. 2019, 6. [CrossRef]

162. Lupan, O.; Cretu, V.; Postica, V.; Ababii, N.; Polonskyi, O.; Kaidas, V.; Schütt, F.; Mishra, Y.K.; Monaico, E.; Tiginyanu, I.; et al. Enhanced ethanol vapour sensing performances of copper oxide nanocrystals with mixed phases. Sens. Actuators B 2016, 224, 434-448. [CrossRef]

163. Xiaofeng, W.; Ma, W.; Sun, K.; Hu, J.; Qin, H. Nanocrystalline $\mathrm{Gd}_{1-\mathrm{x}} \mathrm{Ca}_{\mathrm{x}} \mathrm{FeO}_{3}$ sensors for detection of methanol gas. J. Rare Earths 2017, 35, 690-696. [CrossRef]

164. Wu, Y.; Jiang, T.; Shi, T.; Sun, B.; Tang, Z.; Liao, G. Au modified ZnO nanowires for ethanol gas sensing. Sci. China Technol. Sci. 2017, 60, 71-77. [CrossRef]

165. Choi, K.S.; Park, S.; Chang, S.-P. Enhanced ethanol sensing properties based on $\mathrm{SnO}_{2}$ nanowires coated with $\mathrm{Fe}_{2} \mathrm{O}_{3}$ nanoparticles. Sens. Actuators B 2017, 238, 871-879. [CrossRef]

166. Kim, K.-K.; Kim, D.; Kang, S.-H.; Park, S. Detection of ethanol gas using $\operatorname{In}_{2} \mathrm{O}_{3}$ nanoparticle-decorated ZnS nanowires. Sens. Actuators B 2017, 248, 43-49. [CrossRef]

167. Song, L.; Dou, K.; Wang, R.; Leng, P.; Luo, L.; Xi, Y.; Kaun, C.-C.; Han, N.; Wang, F.; Chen, Y. Sr-Doped Cubic $\mathrm{In}_{2} \mathrm{O}_{3} / \mathrm{Rhombohedral}$ $\mathrm{In}_{2} \mathrm{O}_{3}$ Homojunction Nanowires for Highly Sensitive and Selective Breath Ethanol Sensing: Experiment and DFT Simulation Studies. ACS Appl. Mater. Interfaces 2020, 12, 1270-1279. [CrossRef]

168. Choi, S.; Bonyani, M.; Sun, G.-J.; Lee, J.K.; Hyun, S.K.; Lee, C. $\mathrm{Cr}_{2} \mathrm{O}_{3}$ nanoparticle-functionalized $\mathrm{WO}_{3}$ nanorods for ethanol gas sensors. Appl. Surf. Sci. 2018, 432, 241-249. [CrossRef]

169. Shankar, P.; Rayappan, J.B.B. Room temperature ethanol sensing properties of ZnO nanorods prepared using an electrospinning technique. J. Mater. Chem. C 2017, 5, 10869-10880. [CrossRef]

170. Zhao, S.; Shen, Y.; Yan, X.; Zhou, P.; Yin, Y.; Lu, R.; Han, C.; Cui, B.; Wei, D. Complex-surfactant-assisted hydrothermal synthesis of one-dimensional $\mathrm{ZnO}$ nanorods for high-performance ethanol gas sensor. Sens. Actuators B 2019, 286, 501-511. [CrossRef]

171. Cao, P.; Yang, Z.; Navale, S.T.; Han, S.; Liu, X.; Liu, W.; Lu, Y.; Stadler, F.J.; Zhu, D. Ethanol sensing behavior of Pd-nanoparticles decorated ZnO-nanorod based chemiresistive gas sensors. Sens. Actuators B 2019, 298, 126850. [CrossRef]

172. Yang, X.; Zhang, S.; Yu, Q.; Zhao, L.; Sun, P.; Wang, T.; Liu, F.; Yan, X.; Gao, Y.; Liang, X.; et al. One step synthesis of branched $\mathrm{SnO}_{2} / \mathrm{ZnO}$ heterostructures and their enhanced gas-sensing properties. Sens. Actuators B 2019, 281, 415-423. [CrossRef]

173. Perfecto, T.M.; Zito, C.A.; Mazon, T.; Volanti, D.P. Flexible room-temperature volatile organic compound sensors based on reduced graphene oxide- $\mathrm{WO}_{3} \cdot 0.33 \mathrm{H}_{2} \mathrm{O}$ nano-needles. J. Mater. Chem. C 2018, 6, 2822-2829. [CrossRef]

174. Zhao, Y.; Li, Y.; Wan, W.; Ren, X.; Zhao, H. Surface defect and gas-sensing performance of the well-aligned $\mathrm{Sm}_{-}$-doped $\mathrm{SnO}_{2}$ nanoarrays. Mater. Lett. 2018, 218, 22-26. [CrossRef]

175. Han, T.; Ma, S.Y.; Xu, X.L.; Xu, X.H.; Pei, S.T.; Tie, Y.; Cao, P.F.; Liu, W.W.; Wang, B.J.; Zhang, R.; et al. Rough SmFeO 3 nanofibers as an optimization ethylene glycol gas sensor prepared by electrospinning. Mater. Lett. 2020, 268, 127575. [CrossRef]

176. Feng, C.; Kou, X.; Chen, B.; Qian, G.; Sun, Y.; Lu, G. One-pot synthesis of in doped NiO nanofibers and their gas sensing properties. Sens. Actuators B 2017, 253, 584-591. [CrossRef]

177. Liu, Y.; Yang, P.; Li, J.; Matras-Postolek, K.; Yue, Y.; Huang, B. Formation of $\mathrm{SiO}_{2} @ \mathrm{SnO}_{2}$ core-shell nanofibers and their gas sensing properties. RSC Adv. 2016, 6, 13371-13376. [CrossRef]

178. Jun, L.; Chen, Q.; Fu, W.; Yang, Y.; Zhu, W.; Zhang, J. Electrospun Yb-Doped $\operatorname{In}_{2} \mathrm{O}_{3}$ Nanofiber Field-Effect Transistors for Highly Sensitive Ethanol Sensors. ACS Appl. Mater. Interfaces 2020, 12, 38425-38434. [CrossRef] [PubMed] 


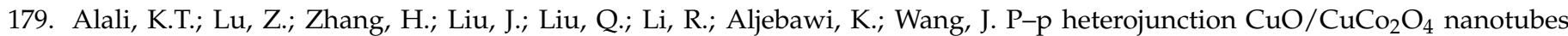
synthesized via electrospinning technology for detecting n-propanol gas at room temperature. Inorg. Chem. Front. 2017, 4, 1219-1230. [CrossRef]

180. Su, C.; Zhang, L.; Han, Y.; Ren, C.; Zeng, M.; Zhou, Z.; Su, Y.; Hu, N.; Wei, H.; Yang, Z. Controllable synthesis of heterostructured $\mathrm{CuO}-\mathrm{NiO}$ nanotubes and their synergistic effect for glycol gas sensing. Sens. Actuators B 2020, 304, 127347. [CrossRef]

181. Zhang, L.; He, J.; Jiao, W. Synthesis and gas sensing performance of $\mathrm{NiO}$ decorated $\mathrm{SnO}_{2}$ vertical-standing nanotubes composite thin films. Sens. Actuators B 2019, 281, 326-334. [CrossRef]

182. Zhao, C.; Gong, H.; Niu, G.; Wang, F. Electrospun Ca-doped $\operatorname{In}_{2} \mathrm{O}_{3}$ nanotubes for ethanol detection with enhanced sensitivity and selectivity. Sens. Actuators B 2019, 299, 126946. [CrossRef]

183. Bai, J.; Wang, Q.; Wang, Y.; Cheng, X.; Yang, Z.; Gu, X.; Huang, B.; Sun, G.; Zhang, Z.; Pan, X.; et al. Role of nickel dopant on gas response and selectivity of electrospun indium oxide nanotubes. J. Colloid Interface Sci. 2020, 560, 447-457. [CrossRef] [PubMed]

184. Wang, Q.; Bai, J.; Hu, Q.; Hao, J.; Cheng, X.; Li, J.; Xie, E.; Wang, Y.; Pan, X. W-doped NiO as a material for selective resistive ethanol sensors. Sens. Actuators B 2020, 308, 127668. [CrossRef]

185. Li, Y.; Yang, H.; Tian, J.; Hu, X.; Cui, H. Synthesis of $\mathrm{In}_{2} \mathrm{O}_{3}$ nanoparticle/ $\mathrm{TiO}_{2}$ nanobelt heterostructures for near room temperature ethanol sensing. RSC Adv. 2017, 7, 11503-11509. [CrossRef]

186. Mo, Y.; Tan, Z.; Sun, L.; Lu, Y.; Liu, X. Ethanol-sensing properties of $\alpha-\mathrm{MoO}_{3}$ nanobelts synthesized by hydrothermal method. J. Alloys Compd. 2020, 812, 152166. [CrossRef]

187. Yang, S.; Liu, Y.; Chen, T.; Jin, W.; Yang, T.; Cao, M.; Liu, S.; Zhou, J.; Zakharova, G.S.; Chen, W. Zn doped MoO 3 nanobelts and the enhanced gas sensing properties to ethanol. Appl. Surf. Sci. 2017, 393, 377-384. [CrossRef]

188. Wang, M.; Hou, T.; Shen, Z.; Zhao, X.; Ji, H. MOF-derived $\mathrm{Fe}_{2} \mathrm{O}_{3}$ : Phase control and effects of phase composition on gas sensing performance. Sens. Actuators B 2019, 292, 171-179. [CrossRef]

189. Nguyen, T.T.D.; Choi, H.-N.; Ahemad, M.J.; Van Dao, D.; Lee, I.-H.; Yu, Y.-T. Hydrothermal synthesis of $\mathrm{In}_{2} \mathrm{O}_{3}$ nanocubes for highly responsive and selective ethanol gas sensing. J. Alloys Compd. 2020, 820, 153133. [CrossRef]

190. Zhu, L.; Zeng, W.; Li, Y.; Yang, J. Enhanced ethanol gas-sensing property based on hollow $\mathrm{MoO}_{3}$ microcages. Phys. E Low-Dimens. Syst. Nanostruct. 2019, 106, 170-175. [CrossRef]

191. Zhang, X.; Lan, W.; Xu, J.; Luo, Y.; Pan, J.; Liao, C.; Yang, L.; Tan, W.; Huang, X. ZIF-8 derived hierarchical hollow ZnO nanocages with quantum dots for sensitive ethanol gas detection. Sens. Actuators B 2019, 289, 144-152. [CrossRef]

192. Zhang, J.; Lu, H.; Zhang, L.; Leng, D.; Zhang, Y.; Wang, W.; Gao, Y.; Lu, H.; Gao, J.; Zhu, G.; et al. Metal-organic frameworkderived $\mathrm{ZnO}$ hollow nanocages functionalized with nanoscale Ag catalysts for enhanced ethanol sensing properties. Sens. Actuators B 2019, 291, 458-469. [CrossRef]

193. Zhou, S.; Chen, M.; Lu, Q.; Hu, J.; Wang, H.; Li, K.; Li, K.; Zhang, J.; Zhu, Z.; Liu, Q. Design of hollow dodecahedral Cu $2 \mathrm{O}$ nanocages for ethanol gas sensing. Mater. Lett. 2019, 247, 15-18. [CrossRef]

194. Cao, F.; Li, C.; Li, M.; Li, H.; Huang, X.; Yang, B. Direct growth of Al-doped ZnO ultrathin nanosheets on electrode for ethanol gas sensor application. Appl. Surf. Sci. 2018, 447, 173-181. [CrossRef]

195. Niu, G.; Zhao, C.; Gong, H.; Yang, Z.; Leng, X.; Wang, F. NiO nanoparticle-decorated $\mathrm{SnO}_{2}$ nanosheets for ethanol sensing with enhanced moisture resistance. Microsyst. Nanoeng. 2019, 5, 21. [CrossRef]

196. Liu, X.; Sun, Y.; Yu, M.; Yin, Y.; Du, B.; Tang, W.; Jiang, T.; Yang, B.; Cao, W.; Ashfold, M.N.R. Enhanced ethanol sensing properties of ultrathin $\mathrm{ZnO}$ nanosheets decorated with $\mathrm{CuO}$ nanoparticles. Sens. Actuators B 2018, 255, 3384-3390. [CrossRef]

197. Bharatula, L.D.; Erande, M.B.; Mulla, I.S.; Rout, C.S.; Late, D.J. $\mathrm{SnS}_{2}$ nanoflakes for efficient humidity and alcohol sensing at room temperature. RSC Adv. 2016, 6, 105421-105427. [CrossRef]

198. Liu, X.-H.; Yin, P.-F.; Kulinich, S.A.; Zhou, Y.-Z.; Mao, J.; Ling, T.; Du, X.-W. Arrays of Ultrathin CdS Nanoflakes with High-Energy Surface for Efficient Gas Detection. ACS Appl. Mater. Interfaces 2017, 9, 602-609. [CrossRef]

199. Darvishnejad, M.H.; Anaraki Firooz, A.; Beheshtian, J.; Khodadadi, A.A. Highly sensitive and selective ethanol and acetone gas sensors by adding some dopants ( $\mathrm{Mn}, \mathrm{Fe}, \mathrm{Co}, \mathrm{Ni}$ ) onto hexagonal $\mathrm{ZnO}$ plates. RSC Adv. 2016, 6, 7838-7845. [CrossRef]

200. Chen, Y.; Li, H.; Ma, Q.; Che, Q.; Wang, J.; Wang, G.; Yang, P. ZIF-8 derived hexagonal-like $\alpha-\mathrm{Fe}_{2} \mathrm{O}_{3} / \mathrm{ZnO} / \mathrm{Au}$ nanoplates with tunable surface heterostructures for superior ethanol gas-sensing performance. Appl. Surf. Sci. 2018, 439, 649-659. [CrossRef]

201. Phuoc, L.H.; Tho, D.D.; Dung, N.T.; Hien, V.X.; Vuong, D.D.; Chien, N.D. Enhancement of ethanol-sensing properties of ZnO nanoplates by UV illumination. Bull. Mater. Sci. 2019, 42, 72. [CrossRef]

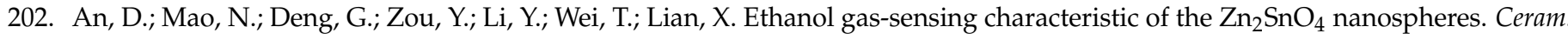
Inter. 2016, 42, 3535-3541. [CrossRef]

203. Wang, C.; Kou, X.; Xie, N.; Guo, L.; Sun, Y.; Chuai, X.; Ma, J.; Sun, P.; Wang, Y.; Lu, G. Detection of Methanol with Fast Response by Monodispersed Indium Tungsten Oxide Ellipsoidal Nanospheres. ACS Sens. 2017, 2, 648-654. [CrossRef] [PubMed]

204. Liu, X.; Sun, X.; Duan, X.; Zhang, C.; Zhao, K.; Xu, X. Core-shell Ag@ $\operatorname{In}_{2} \mathrm{O}_{3}$ hollow hetero-nanostructures for selective ethanol detection in air. Sens. Actuators $B$ 2020, 305, 127450. [CrossRef]

205. Yin, Y.; Shen, Y.; Zhou, P.; Lu, R.; Li, A.; Zhao, S.; Liu, W.; Wei, D.; Wei, K. Fabrication, characterization and n-propanol sensing properties of perovskite-type $\mathrm{ZnSnO}_{3}$ nanospheres based gas sensor. Appl. Surf. Sci. 2020, 509, 145335. [CrossRef]

206. Han, B.; Liu, X.; Xing, X.; Chen, N.; Xiao, X.; Liu, S.; Wang, Y. A high response butanol gas sensor based on ZnO hollow spheres. Sens. Actuators B 2016, 237, 423-430. [CrossRef] 
207. Yang, H.M.; Ma, S.Y.; Yang, G.J.; Jin, W.X.; Wang, T.T.; Jiang, X.H.; Li, W.Q. High sensitive and low concentration detection of methanol by a gas sensor based on one-step synthesis $\alpha-\mathrm{Fe}_{2} \mathrm{O}_{3}$ hollow spheres. Mater. Lett. 2016, 169, 73-76. [CrossRef]

208. Acharyya, D.; Huang, K.Y.; Chattopadhyay, P.P.; Ho, M.S.; Fecht, H.J.; Bhattacharyya, P. Hybrid 3D structures of ZnO nanoflowers and PdO nanoparticles as a highly selective methanol sensor. Analyst 2016, 141, 2977-2989. [CrossRef]

209. Carbone, M.; Tagliatesta, P. NiO Grained-Flowers and Nanoparticles for Ethanol Sensing. Materials 2020, 13, 1880. [CrossRef]

210. Li, B.; Xia, J.; Liu, J.; Liu, Q.; Huang, G.; Zhang, H.; Jing, X.; Li, R.; Wang, J. RGO nanosheets modified NiCo $2 \mathrm{~S}_{4}$ nanoflowers for improved ethanol sensing performance at low temperature. Chem. Phys. Lett. 2018, 703, 80-85. [CrossRef]

211. Maity, I.; Bhattacharyya, P. Potentiallity of Surface Modified $\mathrm{TiO}_{2}$ Nanoflowers for Alcohol Sensing Application. In Proceedings of the 2019 2nd International Symposium on Devices, Circuits and Systems (ISDCS), Higashi-Hiroshima, Japan, 6-8 March 2019; pp. $1-4$.

212. Xing, X.; Li, Y.; Deng, D.; Chen, N.; Liu, X.; Xiao, X.; Wang, Y. Ag-Functionalized macro-/mesoporous AZO synthesized by solution combustion for VOCs gas sensing application. RSC Adv. 2016, 6, 101304-101312. [CrossRef]

213. Xing, X.; Chen, T.; Li, Y.; Deng, D.; Xiao, X.; Wang, Y. Flash synthesis of Al-doping macro-/nanoporous ZnO from self-sustained decomposition of Zn-based complex for superior gas-sensing application to n-butanol. Sens. Actuators B 2016, 237, 90-98. [CrossRef]

214. Wang, Y.; Zhang, B.; Liu, J.; Yang, Q.; Cui, X.; Gao, Y.; Chuai, X.; Liu, F.; Sun, P.; Liang, X.; et al. Au-loaded mesoporous WO 3 : Preparation and n-butanol sensing performances. Sens. Actuators B 2016, 236, 67-76. [CrossRef]

215. Wang, Z.; Tian, Z.; Han, D.; Gu, F. Highly Sensitive and Selective Ethanol Sensor Fabricated with In-Doped 3DOM ZnO. ACS Appl. Mater. Interfaces 2016, 8, 5466-5474. [CrossRef] [PubMed]

216. Saboor, F.H.; Khodadadi, A.A.; Mortazavi, Y.; Asgari, M. Microemulsion synthesized silica/ZnO stable core/shell sensors highly selective to ethanol with minimum sensitivity to humidity. Sens. Actuators B 2017, 238, 1070-1083. [CrossRef]

217. Tomer, V.K.; Malik, R.; Kailasam, K. Near-Room-Temperature Ethanol Detection Using Ag-Loaded Mesoporous Carbon Nitrides. ACS Omega 2017, 2, 3658-3668. [CrossRef] [PubMed]

218. Xiao, L.; Xu, S.; Yu, G.; Liu, S. Efficient hierarchical mixed $\mathrm{Pd} / \mathrm{SnO}_{2}$ porous architecture deposited microheater for low power ethanol gas sensor. Sens. Actuators B 2018, 255, 2002-2010. [CrossRef]

219. Zhang, X.; Xu, G.; Wang, H.; Cui, H.; Zhan, X.; Sang, L.; Zhang, G. Preparation of meso-porous $\mathrm{SnO}_{2}$ fibers with enhanced sensitivity for n-butanol. Ceram. Int. 2018, 44, 4990-4995. [CrossRef]

220. Zhao, T.; Qiu, P.; Fan, Y.; Yang, J.; Jiang, W.; Wang, L.; Deng, Y.; Luo, W. Hierarchical Branched $\mathrm{Mesoporous} \mathrm{TiO}_{2}-\mathrm{SnO}{ }_{2}$ Nanocomposites with Well-Defined n-n Heterojunctions for Highly Efficient Ethanol Sensing. Adv. Sci. 2019, 6, 1902008. [CrossRef]

221. Mo, Y.; Shi, F.; Qin, S.; Tang, P.; Feng, Y.; Zhao, Y.; Li, D. Facile Fabrication of Mesoporous Hierarchical Co-Doped ZnO for Highly Sensitive Ethanol Detection. Ind. Eng. Chem. Res. 2019, 58, 8061-8071. [CrossRef]

222. Wei, Q.; Song, P.; Yang, Z.; Wang, Q. Hierarchical assembly of $\mathrm{Fe}_{2} \mathrm{O}_{3}$ nanorods on $\mathrm{SnO}_{2}$ nanospheres with enhanced ethanol sensing properties. Phys. E Low-Dimens. Syst. Nanostruct. 2018, 103, 156-163. [CrossRef]

223. Li, H.; Zhu, D.; Yang, Z.; Lu, W.; Pu, Y. The ethanol-sensitive property of hierarchical $\mathrm{MoO}_{3}-\mathrm{mixed}_{\mathrm{SnO}} \mathrm{O}_{2}$ aerogels via facile ambient pressure drying. Appl. Surf. Sci. 2019, 489, 384-391. [CrossRef]

224. Wang, X.; Liu, F.; Chen, X.; Song, X.; Xu, G.; Han, Y.; Tian, J.; Cui, H. $\operatorname{In}_{2} \mathrm{O}_{3}$ Nanoparticles Decorated ZnO Hierarchical Structures for n-Butanol Sensor. ACS Appl. Nano Mater. 2020, 3, 3295-3304. [CrossRef]

225. Wang, L.L.; Li, Z.J.; Luo, L.; Zhao, C.Z.; Kang, L.P.; Liu, D.W. Methanol sensing properties of honeycomb-like $\mathrm{SnO}_{2}$ grown on silicon nanoporous pillar array. J. Alloys Compd. 2016, 682, 170-175. [CrossRef]

226. Jiao, Z.; Wang, Y.; Ying, M.; Xu, J.; Xu, L.; Zhang, H. Copolymer-assisted fabrication of rambutan-like $\mathrm{SnO}_{2}$ hierarchical nanostructure with enhanced sensitivity for n-butanol. Mater. Chem. Phys. 2016, 172, 113-120. [CrossRef]

227. Lupan, O.; Postica, V.; Gröttrup, J.; Mishra, A.K.; de Leeuw, N.H.; Carreira, J.F.C.; Rodrigues, J.; Ben Sedrine, N.; Correia, M.R.; Monteiro, T.; et al. Hybridization of Zinc Oxide Tetrapods for Selective Gas Sensing Applications. ACS Appl. Mater. Interfaces 2017, 9, 4084-4099. [CrossRef] [PubMed]

228. Zhao, R.; Wang, Z.; Yang, Y.; Xing, X.; Zou, T.; Wang, Z.; Wang, Y. Raspberry-like $\mathrm{SnO}_{2}$ hollow nanostructure as a high response sensing material of gas sensor toward n-butanol gas. J. Phys. Chem. Solids 2018, 120, 173-182. [CrossRef]

229. Li, Y. A novel snowflake-like $\mathrm{SnO}_{2}$ hierarchical architecture with superior gas sensing properties. Phys. E Low-Dimens. Syst. Nanostruct. 2018, 96, 54-56. [CrossRef]

230. Wang, C.; Zhang, Y.; Sun, X.; Sun, Y.; Liu, F.; Yan, X.; Wang, C.; Sun, P.; Geyu, L. Fast detection of alcohols by novel sea cucumber-like indium tungsten oxide. Sens. Actuators B 2020, 319, 128158. [CrossRef]

231. Zhao, Y.; Liu, Y.; Ma, Y.; Li, Y.; Zhang, J.; Ren, X.; Li, C.; Zhao, J.; Zhu, J.; Zhao, H. Hollow Pentagonal-Cone-Structured SnO 2 Architectures Assembled with Nanorod Arrays for Low-Temperature Ethanol Sensing. ACS Appl. Nano Mater. 2020, 3, 7720-7731. [CrossRef]

232. Qi, T.; Yang, X.; Sun, J. Neck-connected ZnO films derived from core-shell zeolitic imidazolate framework-8 (ZIF-8)@ZnO for highly sensitive ethanol gas sensors. Sens. Actuators B 2019, 283, 93-98. [CrossRef]

233. Zhang, H.; Yi, J. Enhanced ethanol gas sensing performance of $\mathrm{ZnO}$ nanoflowers decorated with $\mathrm{LaMnO}_{3}$ perovskite nanoparticles. Mater. Lett. 2018, 216, 196-198. [CrossRef] 
234. Li, Y.; Deng, D.; Xing, X.; Chen, N.; Liu, X.; Xiao, X.; Wang, Y. A high performance methanol gas sensor based on palladiumplatinum- $\mathrm{In}_{2} \mathrm{O}_{3}$ composited nanocrystalline $\mathrm{SnO}_{2}$. Sens. Actuators B 2016, 237, 133-141. [CrossRef]

235. Zito, C.A.; Perfecto, T.M.; Volanti, D.P. Impact of reduced graphene oxide on the ethanol sensing performance of hollow $\mathrm{SnO}_{2}$ nanoparticles under humid atmosphere. Sens. Actuators B 2017, 244, 466-474. [CrossRef]

236. Muthukumaravel, C.; Karunakaran, U.; Mangamma, G. Local grain-to-grain conductivity in an $\mathrm{SnO}_{2}-\mathrm{V}_{2} \mathrm{O}_{5}$ nanocomposite ethanol sensor. Nanotechnology 2020, 31, 344001. [CrossRef]

237. Postica, V.; Hölken, I.; Schneider, V.; Kaidas, V.; Polonskyi, O.; Cretu, V.; Tiginyanu, I.; Faupel, F.; Adelung, R.; Lupan, O. Multifunctional device based on $\mathrm{ZnO}:$ Fe nanostructured films with enhanced UV and ultra-fast ethanol vapour sensing. Mater. Sci. Semicond. Proc. 2016, 49, 20-33. [CrossRef]

238. Cao, J.; Qin, C.; Wang, Y.; Zhang, H.; Zhang, B.; Gong, Y.; Wang, X.; Sun, G.; Bala, H.; Zhang, Z. Synthesis of g-C $\mathrm{C}_{3} \mathrm{~N}_{4}$ nanosheet modified $\mathrm{SnO}_{2}$ composites with improved performance for ethanol gas sensing. RSC Adv. 2017, 7, 25504-25511. [CrossRef]

239. Li, L.; Zhang, C.; Zhang, R.; Gao, X.; He, S.; Liu, M.; Li, X.; Chen, W. $2 \mathrm{D}$ ultrathin $\mathrm{Co}_{3} \mathrm{O}_{4}$ nanosheet array deposited on 3D carbon foam for enhanced ethanol gas sensing application. Sens. Actuators B 2017, 244, 664-672. [CrossRef]

240. Kukkar, D.; Vellingiri, K.; Kaur, R.; Bhardwaj, S.K.; Deep, A.; Kim, K.-H. Nanomaterials for sensing of formaldehyde in air: Principles, applications, and performance evaluation. Nano Res. 2019, 12, 225-246. [CrossRef]

241. Gu, F.; Li, C.; Han, D.; Wang, Z. Manipulating the Defect Structure (VO) of $\operatorname{In}_{2} \mathrm{O}_{3}$ Nanoparticles for Enhancement of Formaldehyde Detection. ACS Appl. Mater. Interfaces 2018, 10, 933-942. [CrossRef]

242. Hussain, M.; Kotova, K.; Lieberzeit, P.A. Molecularly Imprinted Polymer Nanoparticles for Formaldehyde Sensing with QCM. Sensors 2016, 16, 1011. [CrossRef]

243. Xiang, D.L.; Hou, S.M.; Tong, D.G. Amorphous $\mathrm{Eu}_{0.9} \mathrm{Ni}_{0.1} \mathrm{~B}_{6}$ Nanoparticles for Formaldehyde Vapor Detection. ACS Appl. Nano Mater. 2019, 2, 4048-4052. [CrossRef]

244. Hu, J.; Wang, T.; Wang, Y.; Huang, D.; He, G.; Han, Y.; Hu, N.; Su, Y.; Zhou, Z.; Zhang, Y.; et al. Enhanced formaldehyde detection based on Ni doping of $\mathrm{SnO}_{2}$ nanoparticles by one-step synthesis. Sens. Actuators B 2018, 263, 120-128. [CrossRef]

245. Prajesh, R.; Goyal, V.; Nahid, M.; Saini, V.; Singh, A.K.; Sharma, A.K.; Bhargava, J.; Agarwal, A. Nickel oxide (NiO) thin film optimization by reactive sputtering for highly sensitive formaldehyde sensing. Sens. Actuators B 2020, 318, 128166. [CrossRef]

246. Castro-Hurtado, I.; Gonzalez-Chávarri, J.; Morandi, S.; Samà, J.; Romano-Rodríguez, A.; Castaño, E.; Mandayo, G.G. Formaldehyde sensing mechanism of $\mathrm{SnO}_{2}$ nanowires grown on-chip by sputtering techniques. RSC Adv. 2016, 6, 18558-18566. [CrossRef]

247. Zhu, L.-Y.; Yuan, K.; Yang, J.-G.; Ma, H.-P.; Wang, T.; Ji, X.-M.; Feng, J.-J.; Devi, A.; Lu, H.-L. Fabrication of heterostructured $\mathrm{p}-\mathrm{CuO} / \mathrm{n}-\mathrm{SnO}_{2}$ core-shell nanowires for enhanced sensitive and selective formaldehyde detection. Sens. Actuators B 2019, 290, 233-241. [CrossRef]

248. Chen, L.; Cui, J.; Sheng, X.; Xie, T.; Xu, T.; Feng, X. High-Performance Photoelectronic Sensor Using Mesostructured ZnO Nanowires. ACS Sens. 2017, 2, 1567-1572. [CrossRef]

249. Song, L.; Luo, L.; Xi, Y.; Song, J.; Wang, Y.; Yang, L.; Wang, A.; Chen, Y.; Han, N.; Wang, F. Reduced Graphene Oxide-Coated Si Nanowires for Highly Sensitive and Selective Detection of Indoor Formaldehyde. Nanoscale Res. Lett. 2019, 14, 97. [CrossRef]

250. Wang, Z.; Hou, C.; De, Q.; Gu, F.; Han, D. One-Step Synthesis of Co-Doped $\operatorname{In}_{2} \mathrm{O}_{3}$ Nanorods for High Response of Formaldehyde Sensor at Low Temperature. ACS Sens. 2018, 3, 468-475. [CrossRef]

251. Zhang, X.; Song, D.; Liu, Q.; Chen, R.; Hou, J.; Liu, J.; Zhang, H.; Yu, J.; Liu, P.; Wang, J. Designed synthesis of Ag-functionalized Ni-doped $\mathrm{In}_{2} \mathrm{O}_{3}$ nanorods with enhanced formaldehyde gas sensing properties. J. Mater. Chem. C 2019, 7, 7219-7229. [CrossRef]

252. Zhang, Y.; Zhang, J.; Zhao, J.; Zhu, Z.; Liu, Q. Ag-LaFeO 3 fibers, spheres, and cages for ultrasensitive detection of formaldehyde at low operating temperatures. Phys. Chem. Chem. Phys. 2017, 19, 6973-6980. [CrossRef]

253. Wei, W.; Guo, S.; Chen, C.; Sun, L.; Chen, Y.; Guo, W.; Ruan, S. High sensitive and fast formaldehyde gas sensor based on Ag-doped $\mathrm{LaFeO}_{3}$ nanofibers. J. Alloys Compd. 2017, 695, 1122-1127. [CrossRef]

254. Gao, X.; Li, F.; Wang, R.; Zhang, T. A formaldehyde sensor: Significant role of p-n heterojunction in gas-sensitive core-shell nanofibers. Sens. Actuators B 2018, 258, 1230-1241. [CrossRef]

255. Li, H.; Chu, S.; Ma, Q.; Wang, J.; Che, Q.; Wang, G.; Yang, P. Hierarchical $\mathrm{WO}_{3} / \mathrm{ZnWO}_{4} 1 \mathrm{D}$ fibrous heterostructures with tunable in-situ growth of $\mathrm{WO}_{3}$ nanoparticles on surface for efficient low concentration HCHO detection. Sens. Actuators B 2019, 286, 564-574. [CrossRef]

256. Tie, Y.; Ma, S.Y.; Pei, S.T.; Zhang, Q.X.; Zhu, K.M.; Zhang, R.; Xu, X.H.; Han, T.; Liu, W.W. Pr doped BiFeO 3 hollow nanofibers via electrospinning method as a formaldehyde sensor. Sens. Actuators B 2020, 308, 127689. [CrossRef]

257. Liang, Q.; Zou, X.; Chen, H.; Fan, M.; Li, G.-D. High-performance formaldehyde sensing realized by alkaline-earth metals doped $\mathrm{In}_{2} \mathrm{O}_{3}$ nanotubes with optimized surface properties. Sens. Actuators B 2020, 304, 127241. [CrossRef]

258. Li, S.; Liu, Y.; Wu, Y.; Chen, W.; Qin, Z.; Gong, N.; Yu, D. Highly sensitive formaldehyde resistive sensor based on a single Er-doped $\mathrm{SnO}_{2}$ nanobelt. Phys. B Condens. Matter 2016, 489, 33-38. [CrossRef]

259. Fu, X.; Yang, P.; Xiao, X.; Zhou, D.; Huang, R.; Zhang, X.; Cao, F.; Xiong, J.; Hu, Y.; Tu, Y.; et al. Ultra-fast and highly selective room-temperature formaldehyde gas sensing of Pt-decorated $\mathrm{MoO}_{3}$ nanobelts. J. Alloys Compd. 2019, 797, 666-675. [CrossRef]

260. Zhou, T.; Zhang, T.; Zhang, R.; Lou, Z.; Deng, J.; Wang, L. Hollow ZnSnO 3 Cubes with Controllable Shells Enabling Highly Efficient Chemical Sensing Detection of Formaldehyde Vapors. ACS Appl. Mater. Interfaces 2017, 9, 14525-14533. [CrossRef]

261. Du, L.; Gu, K.; Zhu, M.; Zhang, J.; Zhang, M. Perovskite-type $\mathrm{ZnSn}(\mathrm{OH})_{6}$ hollow cubes with controllable shells for enhanced formaldehyde sensing performance at low temperature. Sens. Actuators B 2019, 288, 298-306. [CrossRef] 
262. Zhang, N.; Ruan, S.; Qu, F.; Yin, Y.; Li, X.; Wen, S.; Adimi, S.; Yin, J. Metal-organic framework-derived $\mathrm{Co}_{3} \mathrm{O}_{4} / \mathrm{CoFe}_{2} \mathrm{O}_{4}$ double-shelled nanocubes for selective detection of sub-ppm-level formaldehyde. Sens. Actuators B 2019, 298, 126887. [CrossRef]

263. Cao, Y.; He, Y.; Zou, X.; Li, G.-D. Tungsten oxide clusters decorated ultrathin $\operatorname{In}_{2} \mathrm{O}_{3}$ nanosheets for selective detecting formaldehyde. Sens. Actuators B 2017, 252, 232-238. [CrossRef]

264. Xu, R.; Zhang, L.-X.; Li, M.-W.; Yin, Y.-Y.; Yin, J.; Zhu, M.-Y.; Chen, J.-J.; Wang, Y.; Bie, L.-J. Ultrathin SnO 2 nanosheets with dominant high-energy $\{001\}$ facets for low temperature formaldehyde gas sensor. Sens. Actuators B 2019, 289, 186-194. [CrossRef]

265. Kim, E.-B.; Seo, H.-K. Highly Sensitive Formaldehyde Detection Using Well-Aligned Zinc Oxide Nanosheets Synthesized by Chemical Bath Deposition Technique. Materials 2019, 12, 250. [CrossRef] [PubMed]

266. Gu, F.; Di, M.; Han, D.; Hong, S.; Wang, Z. Atomically Dispersed Au on $\operatorname{In}_{2} \mathrm{O}_{3}$ Nanosheets for Highly Sensitive and Selective Detection of Formaldehyde. ACS Sens. 2020, 5, 2611-2619. [CrossRef]

267. Li, G.; Cheng, Z.; Xiang, Q.; Yan, L.; Wang, X.; Xu, J. Bimetal PdAu decorated $\mathrm{SnO}_{2}$ nanosheets based gas sensor with temperaturedependent dual selectivity for detecting formaldehyde and acetone. Sens. Actuators B 2019, 283, 590-601. [CrossRef]

268. Hayashi, K.; Kataoka, M.; Jippo, H.; Ohfuchi, M.; Sato, S. Vacancy-Assisted Selective Detection of Low-ppb Formaldehyde in Two-Dimensional Layered SnS 2 . ACS Appl. Mater. Interfaces 2020, 12, 12207-12214. [CrossRef] [PubMed]

269. Hussain, S.; Liu, T.; Javed, M.S.; Aslam, N.; Zeng, W. Highly reactive 0D ZnS nanospheres and nanoparticles for formaldehyde gas-sensing properties. Sens. Actuators B 2017, 239, 1243-1250. [CrossRef]

270. Zhang, R.; Ma, S.Y.; Zhang, Q.X.; Zhu, K.M.; Tie, Y.; Pei, S.T.; Wang, B.J.; Zhang, J.L. Highly sensitive formaldehyde gas sensors based on Ag doped $\mathrm{Zn}_{2} \mathrm{SnO}_{4} / \mathrm{SnO}_{2}$ hollow nanospheres. Mater. Lett. 2019, 254, 178-181. [CrossRef]

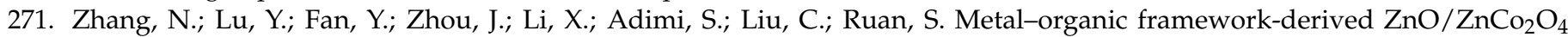
microspheres modified by catalytic PdO nanoparticles for sub-ppm-level formaldehyde detection. Sens. Actuators B 2020, 315, 128118. [CrossRef]

272. Hu, J.; Wang, H.; Chen, M.; Zhang, Y.; Zhao, X.; Zhang, D.; Lu, Q.; Zhang, J.; Liu, Q. Constructing hierarchical SnO 2 nanoflowers for enhanced formaldehyde sensing performances. Mater. Lett. 2020, 263, 126843. [CrossRef]

273. Yin, F.; Li, Y.; Yue, W.; Gao, S.; Zhang, C.; Chen, Z. $\mathrm{Sn}_{3} \mathrm{O}_{4} / \mathrm{rGO}$ heterostructure as a material for formaldehyde gas sensor with a wide detecting range and low operating temperature. Sens. Actuators B 2020, 312, 127954. [CrossRef]

274. Zhang, S.; Song, P.; Li, J.; Zhang, J.; Yang, Z.; Wang, Q. Facile approach to prepare hierarchical Au-loaded $\mathrm{In}_{2} \mathrm{O}_{3}$ porous nanocubes and their enhanced sensing performance towards formaldehyde. Sens. Actuators B 2017, 241, 1130-1138. [CrossRef]

275. Xing, X.; Xiao, X.; Wang, L.; Wang, Y. Highly sensitive formaldehyde gas sensor based on hierarchically porous Ag-loaded ZnO heterojunction nanocomposites. Sens. Actuators B 2017, 247, 797-806. [CrossRef]

276. Malik, R.; Tomer, V.K.; Dankwort, T.; Mishra, Y.K.; Kienle, L. Cubic mesoporous Pd- $\mathrm{WO}_{3}$ loaded graphitic carbon nitride (g-CN) nanohybrids: Highly sensitive and temperature dependent VOC sensors. J. Mater. Chem. A 2018, 6, 10718-10730. [CrossRef]

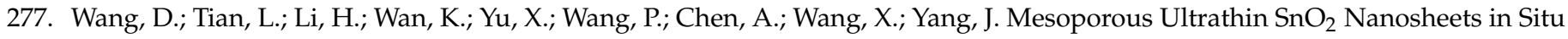
Modified by Graphene Oxide for Extraordinary Formaldehyde Detection at Low Temperatures. ACS Appl. Mater. Interfaces 2019, 11, 12808-12818. [CrossRef] [PubMed]

278. Wang, B.; Yu, J.; Li, X.; Yin, J.; Chen, M. Synthesis and high formaldehyde sensing properties of quasi two-dimensional mesoporous $\mathrm{ZnSnO}_{3}$ nanomaterials. RSC Adv. 2019, 9, 14809-14816. [CrossRef]

279. Yang, K.; Ma, J.; Qiao, X.; Cui, Y.; Jia, L.; Wang, H. Hierarchical porous $\mathrm{LaFeO}_{3}$ nanostructure for efficient trace detection of formaldehyde. Sens. Actuators B 2020, 313, 128022. [CrossRef]

280. Zhang, R.; Ma, S.Y.; Zhang, J.L.; Wang, B.J.; Pei, S.T. Enhanced formaldehyde gas sensing performance based on Bi doped $\mathrm{Zn}_{2} \mathrm{SnO}_{4} / \mathrm{SnO}_{2}$ porous nanospheres. J. Alloys Compd. 2020, 828, 154408. [CrossRef]

281. Chen, H.; Li, C.; Zhang, X.; Yang, W. ZnO nanoplates with abundant porosity for significant formaldehyde-sensing. Mater. Lett. 2020, 260, 126982. [CrossRef]

282. Liu, D.; Wan, J.; Wang, H.; Pang, G.; Tang, Z. Mesoporous Au@ZnO flower-like nanostructure for enhanced formaldehyde sensing performance. Inorg. Chem. Commun. 2019, 102, 203-209. [CrossRef]

283. Zhang, B.; Li, Y.; Luo, N.; Xu, X.; Sun, G.; Wang, Y.; Cao, J. $\mathrm{TiO}_{2} / \mathrm{ZnCo}_{2} \mathrm{O}_{4}$ porous nanorods: Synthesis and temperature-dependent dual selectivity for sensing HCHO and TEA. Sens. Actuators B 2020, 321, 128461. [CrossRef]

284. Shu, S.; Wang, M.; Yang, W.; Liu, S. Synthesis of surface layered hierarchical octahedral-like structured $\mathrm{Zn}_{2} \mathrm{SnO}_{4} / \mathrm{SnO}_{2}$ with excellent sensing properties toward HCHO. Sens. Actuators B 2017, 243, 1171-1180. [CrossRef]

285. Huang, S.; Cheng, B.; Yu, J.; Jiang, C. Hierarchical Pt/ $\mathrm{MnO}_{2}-\mathrm{Ni}(\mathrm{OH})_{2}$ Hybrid Nanoflakes with Enhanced Room-Temperature Formaldehyde Oxidation Activity. ACS Sustain. Chem. Eng. 2018, 6, 12481-12488. [CrossRef]

286. Wang, D.; Wan, K.; Zhang, M.; Li, H.; Wang, P.; Wang, X.; Yang, J. Constructing hierarchical SnO 2 nanofiber/nanosheets for efficient formaldehyde detection. Sens. Actuators B 2019, 283, 714-723. [CrossRef]

287. Wan, K.; Wang, D.; Wang, F.; Li, H.; Xu, J.; Wang, X.; Yang, J. Hierarchical $\mathrm{In}_{2} \mathrm{O}_{3} @ \mathrm{SnO}_{2}$ Core-Shell Nanofiber for High Efficiency Formaldehyde Detection. ACS Appl. Mater. Interfaces 2019, 11, 45214-45225. [CrossRef]

288. Yu, H.; Yang, T.; Wang, Z.; Li, Z.; Xiao, B.; Zhao, Q.; Zhang, M. Facile synthesis cedar-like $\mathrm{SnO}_{2}$ hierarchical micro-nanostructures with improved formaldehyde gas sensing characteristics. J. Alloys Compd. 2017, 724, 121-129. [CrossRef]

289. Tao, Z.; Li, Y.; Zhang, B.; Sun, G.; Xiao, M.; Bala, H.; Cao, J.; Zhang, Z.; Wang, Y. Synthesis of urchin-like $\mathrm{In}_{2} \mathrm{O}_{3}$ hollow spheres for selective and quantitative detection of formaldehyde. Sens. Actuators B 2019, 298, 126889. [CrossRef] 
290. Li, C.; Liu, Y.; Wan, W.; Li, Y.; Ma, Y.; Zhang, J.; Ren, X.; Zhao, H. Hydrothermal synthesis of novel porous butterfly-like hierarchical $\mathrm{SnO}_{2}$ architecture with excellent gas-sensing performance to acetaldehyde. Sens. Actuators B 2020, 318, 128209. [CrossRef]

291. Wang, B.J.; Ma, S.Y.; Pei, S.T.; Xu, X.L.; Cao, P.F.; Zhang, J.L.; Zhang, R.; Xu, X.H.; Han, T. High specific surface area SnO 2 prepared by calcining Sn-MOFs and their formaldehyde-sensing characteristics. Sens. Actuators B 2020, 321, 128560. [CrossRef]

292. Xu, Y.; Tian, X.; Fan, Y.; Sun, Y. A formaldehyde gas sensor with improved gas response and sub-ppm level detection limit based on $\mathrm{NiO} / \mathrm{NiFe}_{2} \mathrm{O}_{4}$ composite nanotetrahedrons. Sens. Actuators B 2020, 309, 127719. [CrossRef]

293. Bo, Z.; Yuan, M.; Mao, S.; Chen, X.; Yan, J.; Cen, K. Decoration of vertical graphene with tin dioxide nanoparticles for highly sensitive room temperature formaldehyde sensing. Sens. Actuators B 2018, 256, 1011-1020. [CrossRef]

294. Wang, J.; Zhan, D.; Wang, K.; Hang, W. The detection of formaldehyde using microelectromechanical acoustic resonator with multiwalled carbon nanotubes-polyethyleneimine composite coating. J. Micromech. Microeng. 2017, 28, 015003. [CrossRef]

295. Nasriddinov, A.; Rumyantseva, M.; Marikutsa, A.; Gaskov, A.; Lee, J.-H.; Kim, J.-H.; Kim, J.-Y.; Kim, S.S.; Kim, H.W. Sub-ppm Formaldehyde Detection by n-n $\mathrm{TiO}_{2} @ \mathrm{SnO}_{2}$ Nanocomposites. Sensors 2019, 19, 3182. [CrossRef] [PubMed]

296. Yang, Y.; Wang, X.; Yi, G.; Li, H.; Shi, C.; Sun, G.; Zhang, Z. Hydrothermal Synthesis of Co3O4/ZnO Hybrid Nanoparticles for Triethylamine Detection. Nanomaterials 2019, 9, 1599. [CrossRef] [PubMed]

297. Zhu, M.; Yang, T.; Zhai, C.; Du, L.; Zhang, J.; Zhang, M. Fast triethylamine gas sensing response properties of Ho-doped SnO 2 nanoparticles. J. Alloys Compd. 2020, 817, 152724. [CrossRef]

298. Liu, H.; Cao, X.; Wu, H.; Li, B.; Li, Y.; Zhu, W.; Yang, Z.; Huang, Y. Innovative development on a p-type delafossite CuCrO 2 nanoparticles based triethylamine sensor. Sens. Actuators B 2020, 324, 128743. [CrossRef]

299. Xu, Y.; Ma, T.; Zhao, Y.; Zheng, L.; Liu, X.; Zhang, J. Multi-metal functionalized tungsten oxide nanowires enabling ultra-sensitive detection of triethylamine. Sens. Actuators B 2019, 300, 127042. [CrossRef]

300. Wang, L.; Li, J.; Wang, Y.; Yu, K.; Tang, X.; Zhang, Y.; Wang, S.; Wei, C. Construction of $1 \mathrm{D}$ SnO ${ }_{2}$-coated ZnO nanowire heterojunction for their improved n-butylamine sensing performances. Sci. Rep. 2016, 6, 35079. [CrossRef]

301. Zhang, H.; Luo, Y.; Zhuo, M.; Yang, T.; Liang, J.; Zhang, M.; Ma, J.; Duan, H.; Li, Q. Diethylamine gas sensor using $\mathrm{V}_{2} \mathrm{O}_{5}-\mathrm{decorated}$ $\alpha-\mathrm{Fe}_{2} \mathrm{O}_{3}$ nanorods as a sensing material. RSC Adv. 2016, 6, 6511-6515. [CrossRef]

302. Liu, L.; Song, P.; Yang, Z.; Wang, Q. Highly sensitive and selective trimethylamine sensors based on $\mathrm{WO}_{3}$ nanorods decorated with Au nanoparticles. Phys. E Low-Dimens. Syst. Nanostruct. 2017, 90, 109-115. [CrossRef]

303. He, K.; He, S.; Yang, W.; Tian, Q. Ag nanoparticles-decorated $\alpha-\mathrm{MoO}_{3}$ nanorods for remarkable and rapid triethylamine-sensing response boosted by pulse-heating technique. J. Alloys Compd. 2019, 808, 151704. [CrossRef]

304. Li, W.; He, S.; Feng, L.; Yang, W. Cr-doped $\alpha-\mathrm{MoO}_{3}$ nanorods for the fast detection of triethylamine using a pulse-heating strategy. Mater. Lett. 2019, 250, 143-146. [CrossRef]

305. He, S.; Li, W.; Feng, L.; Yang, W. Rational interaction between the aimed gas and oxide surfaces enabling high-performance sensor: The case of acidic $\alpha-\mathrm{MoO}_{3}$ nanorods for selective detection of triethylamine. J. Alloys Compd. 2019, 783, 574-582. [CrossRef]

306. Yang, C.; Xu, Y.; Zheng, L.; Zhao, Y.; Zheng, W.; Liu, X.; Zhang, J. Hierarchical $\mathrm{NiCo}_{2} \mathrm{O}_{4}$ microspheres assembled by nanorods with p-type response for detection of triethylamine. Chin. Chem. Lett. 2020, 31, 2077-2082. [CrossRef]

307. Xu, H.; Li, W.; Han, R.; Zhai, T.; Yu, H.; Chen, Z.; Wu, X.; Wang, J.; Cao, B. Enhanced triethylamine sensing properties by fabricating $\mathrm{Au} @ \mathrm{SnO}_{2} / \alpha-\mathrm{Fe}_{2} \mathrm{O}_{3}$ core-shell nanoneedles directly on alumina tubes. Sens. Actuators B 2018, 262, 70-78. [CrossRef]

308. Guo, L.; Wang, C.; Kou, X.; Xie, N.; Liu, F.; Zhang, H.; Liang, X.; Gao, Y.; Sun, Y.; Chuai, X.; et al. Detection of triethylamine with fast response by $\mathrm{Al}_{2} \mathrm{O}_{3} / \alpha-\mathrm{Fe}_{2} \mathrm{O}_{3}$ composite nanofibers. Sens. Actuators B 2018, 266, 139-148. [CrossRef]

309. Ma, Q.; Li, H.; Chu, S.; Liu, Y.; Liu, M.; Fu, X.; Li, H.; Guo, J. $\mathrm{In}_{2} \mathrm{O}_{3}$ Hierarchical Structures of One-Dimensional Electrospun Fibers with in Situ Growth of Octahedron-like Particles with Superior Sensitivity for Triethylamine at Near Room Temperature. ACS Sustain. Chem. Eng. 2020, 8, 5240-5250. [CrossRef]

310. Perillo, P.M.; Rodríguez, D.F. Low temperature trimethylamine flexible gas sensor based on $\mathrm{TiO}_{2}$ membrane nanotubes. J. Alloys Compd. 2016, 657, 765-769. [CrossRef]

311. Paoletti, C.; He, M.; Salvo, P.; Melai, B.; Calisi, N.; Mannini, M.; Cortigiani, B.; Bellagambi, F.G.; Swager, T.M.; Di Francesco, F.; et al. Room temperature amine sensors enabled by sidewall functionalization of single-walled carbon nanotubes. RSC Adv. 2018, 8, 5578-5585. [CrossRef]

312. Galstyan, V.; Ponzoni, A.; Kholmanov, I.; Natile, M.M.; Comini, E.; Sberveglieri, G. Highly sensitive and selective detection of dimethylamine through $\mathrm{Nb}$-doping of $\mathrm{TiO}_{2}$ nanotubes for potential use in seafood quality control. Sens. Actuators B 2020, 303, 127217. [CrossRef]

313. Zhang, J.; Song, P.; Li, Z.; Zhang, S.; Yang, Z.; Wang, Q. Enhanced trimethylamine sensing performance of single-crystal MoO 3 nanobelts decorated with Au nanoparticles. J. Alloys Compd. 2016, 685, 1024-1033. [CrossRef]

314. Li, Z.; Wang, W.; Zhao, Z.; Liu, X.; Song, P. Facile synthesis and enhanced trimethylamine sensing performances of W-doped $\mathrm{MoO}_{3}$ nanobelts. Mater. Sci. Semicond. Proc. 2017, 66, 33-38. [CrossRef]

315. Wei, Q.; Song, P.; Li, Z.; Yang, Z.; Wang, Q. Enhanced triethylamine sensing performance of $\mathrm{MoO}_{3}$ nanobelts by $\mathrm{RuO}_{2}$ nanoparticles decoration. Vacuum 2019, 162, 85-91. [CrossRef]

316. Yang, T.; Gu, K.; Zhu, M.; Lu, Q.; Zhai, C.; Zhao, Q.; Yang, X.; Zhang, M. ZnO-SnO 2 heterojunction nanobelts: Synthesis and ultraviolet light irradiation to improve the triethylamine sensing properties. Sens. Actuators B 2019, 279, 410-417. [CrossRef] 


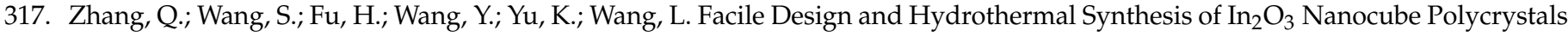
with Superior Triethylamine Sensing Properties. ACS Omega 2020, 5, 11466-11472. [CrossRef]

318. Chen, G.; Chu, X.; Qiao, H.; Ye, M.; Chen, J.; Gao, C.; Guo, C.-Y. Thickness controllable single-crystal WO 3 nanosheets: Highly selective sensor for triethylamine detection at room temperature. Mater. Lett. 2018, 226, 59-62. [CrossRef]

319. Zhai, T.; Xu, H.; Li, W.; Yu, H.; Chen, Z.; Wang, J.; Cao, B. Low-temperature in-situ growth of $\mathrm{SnO}_{2}$ nanosheets and its high triethylamine sensing response by constructing Au-loaded $\mathrm{ZnO} / \mathrm{SnO}_{2}$ heterostructure. J. Alloys Compd. 2018, 737, 603-612. [CrossRef]

320. Wang, X.; Li, Y.; Li, Z.; Zhang, S.; Deng, X.; Zhao, G.; Xu, X. Highly sensitive and low working temperature detection of trace triethylamine based on $\mathrm{TiO}_{2}$ nanoparticles decorated $\mathrm{CuO}$ nanosheets sensors. Sens. Actuators B 2019, 301, 127019. [CrossRef]

321. Bi, W.; Wang, W.; Liu, S. Synthesis of $\mathrm{Rh}-\mathrm{SnO}_{2}$ nanosheets and ultra-high triethylamine sensing performance. J. Alloys Compd. 2020, 817, 152730. [CrossRef]

322. Yan, Y.; Liu, J.; Liu, Q.; Yu, J.; Chen, R.; Zhang, H.; Song, D.; Yang, P.; Zhang, M.; Wang, J. Ag-modified hexagonal nanoflakestextured hollow octahedron $\mathrm{Zn}_{2} \mathrm{SnO}_{4}$ with enhanced sensing properties for triethylamine. J. Alloys Compd. 2020, 823, 153724. [CrossRef]

323. Yang, J.; Wang, S.; Zhang, L.; Dong, R.; Zhu, Z.; Gao, X. $\mathrm{Zn}_{2} \mathrm{SnO}_{4}$-doped $\mathrm{SnO}_{2}$ hollow spheres for phenylamine gas sensor application. Sens. Actuators B 2017, 239, 857-864. [CrossRef]

324. Xue, D.; Wang, Y.; Cao, J.; Zhang, Z. Hydrothermal Synthesis of $\mathrm{CeO}_{2}-\mathrm{SnO}_{2}$ Nanoflowers for Improving Triethylamine Gas Sensing Property. Nanomaterials 2018, 8, 1025. [CrossRef] [PubMed]

325. Tomer, V.K.; Devi, S.; Malik, R.; Nehra, S.P.; Duhan, S. Highly sensitive and selective volatile organic amine (VOA) sensors using mesoporous $\mathrm{WO}_{3}-\mathrm{SnO}_{2}$ nanohybrids. Sens. Actuators B 2016, 229, 321-330. [CrossRef]

326. Wu, Y.-P.; Zhou, W.; Dong, W.-W.; Zhao, J.; Qiao, X.-Q.; Hou, D.-F.; Li, D.-S.; Zhang, Q.; Feng, P. Temperature-Controlled Synthesis of Porous CuO Particles with Different Morphologies for Highly Sensitive Detection of Triethylamine. Cryst. Growth Des. 2017, 17, 2158-2165. [CrossRef]

327. Zhang, S.; Song, P.; Tian, Z.; Wang, Q. Synthesis of mesoporous $\operatorname{In}_{2} \mathrm{O}_{3}$ nanocubes and their superior trimethylamine sensing properties. Mater. Sci. Semicond. Proc. 2018, 75, 58-64. [CrossRef]

328. Zito, C.A.; Perfecto, T.M.; Volanti, D.P. Porous $\mathrm{CeO}_{2}$ nanospheres for a room temperature triethylamine sensor under high humidity conditions. New J. Chem. 2018, 42, 15954-15961. [CrossRef]

329. Song, X.; Xu, Q.; Zhang, T.; Song, B.; Li, C.; Cao, B. Room-temperature, high selectivity and low-ppm-level triethylamine sensor assembled with $\mathrm{Au}$ decahedrons-decorated porous $\alpha-\mathrm{Fe}_{2} \mathrm{O}_{3}$ nanorods directly grown on flat substrate. Sens. Actuators $B \mathbf{2 0 1 8}$ 268, 170-181. [CrossRef]

330. Luo, N.; Sun, G.; Zhang, B.; Li, Y.; Jin, H.; Lin, L.; Bala, H.; Cao, J.; Zhang, Z.; Wang, Y. Improved TEA sensing performance of $\mathrm{ZnCo}_{2} \mathrm{O}_{4}$ by structure evolution from porous nanorod to single-layer nanochain. Sens. Actuators B 2018, 277, 544-554. [CrossRef]

331. Zhao, Y.; Yuan, X.; Sun, Y.; Wang, Q.; Xia, X.-Y.; Tang, B. Facile synthesis of tortoise shell-like porous $\mathrm{NiCo}_{2} \mathrm{O}_{4}$ nanoplate with promising triethylamine gas sensing properties. Sens. Actuators B 2020, 323, 128663. [CrossRef]

332. Xu, Y.; Zheng, L.; Yang, C.; Zheng, W.; Liu, X.; Zhang, J. Oxygen Vacancies Enabled Porous $\mathrm{SnO}_{2}$ Thin Films for Highly Sensitive Detection of Triethylamine at Room Temperature. ACS Appl. Mater. Interfaces 2020, 12, 20704-20713. [CrossRef]

333. Wei, Q.; Sun, J.; Song, P.; Li, J.; Yang, Z.; Wang, Q. Spindle-like $\mathrm{Fe}_{2} \mathrm{O}_{3} / \mathrm{ZnFe}_{2} \mathrm{O}_{4}$ porous nanocomposites derived from metalorganic frameworks with excellent sensing performance towards triethylamine. Sens. Actuators B 2020, 317, 128205. [CrossRef]

334. Meng, F.; Zheng, H.; Sun, Y.; Li, M.; Liu, J. Trimethylamine Sensors Based on Au-Modified Hierarchical Porous Single-Crystalline ZnO Nanosheets. Sensors 2017, 17, 1478. [CrossRef]

335. Yang, T.; Du, L.; Zhai, C.; Li, Z.; Zhao, Q.; Luo, Y.; Xing, D.; Zhang, M. Ultrafast response and recovery trimethylamine sensor based on $\alpha-\mathrm{Fe}_{2} \mathrm{O}_{3}$ snowflake-like hierarchical architectures. J. Alloys Compd. 2017, 718, 396-404. [CrossRef]

336. Liu, F.; Chen, X.; Wang, X.; Han, Y.; Song, X.; Tian, J.; He, X.; Cui, H. Fabrication of $1 \mathrm{D} \mathrm{Zn}{ }_{2} \mathrm{SnO}_{4}$ nanowire and $2 \mathrm{D}$ ZnO nanosheet hybrid hierarchical structures for use in triethylamine gas sensors. Sens. Actuators B 2019, 291, 155-163. [CrossRef]

337. Hou, X.; Wang, Z.; Fan, G.; Ji, H.; Yi, S.; Li, T.; Wang, Y.; Zhang, Z.; Yuan, L.; Zhang, R.; et al. Hierarchical three-dimensional $\mathrm{MoS}_{2} / \mathrm{GO}$ hybrid nanostructures for triethylamine-sensing applications with high sensitivity and selectivity. Sens. Actuators $B$ 2020, 317, 128236. [CrossRef]

338. Jin, H.; Sun, G.; Zhang, B.; Luo, N.; Li, Y.; Lin, L.; Bala, H.; Cao, J.; Zhang, Z.; Wang, Y. Facile synthesis of $\mathrm{Co}_{3} \mathrm{O}_{4}$ nanochains and their improved TEA sensing performance by decorating with Au nanoparticles. J. Alloys Compd. 2019, 776, 782-790. [CrossRef]

339. Han, Y.; Liu, Y.; Su, C.; Chen, X.; Zeng, M.; Hu, N.; Su, Y.; Zhou, Z.; Wei, H.; Yang, Z. Sonochemical synthesis of hierarchical WO 3 flower-like spheres for highly efficient triethylamine detection. Sens. Actuators B 2020, 306, 127536. [CrossRef]

340. Ma, Q.; Fang, Y.; Liu, Y.; Song, J.; Fu, X.; Li, H.; Chu, S.; Chen, Y. Facile synthesis of ZnO morphological evolution with tunable growth habits: Achieving superior gas-sensing properties of hemispherical $\mathrm{ZnO} / \mathrm{Au}$ heterostructures for triethylamine. Phys. $E$ Low-Dimens. Syst. Nanostruct. 2019, 106, 180-186. [CrossRef]

341. Liu, L.; Zhao, Y.; Song, P.; Yang, Z.; Wang, Q. ppb level triethylamine detection of yolk-shell SnO $\mathrm{O}_{2} / \mathrm{Au} / \mathrm{Fe}_{2} \mathrm{O}_{3}$ nanoboxes at low-temperature. Appl. Surf. Sci. 2019, 476, 391-401. [CrossRef]

342. Tao, Z.; Li, Y.; Sun, G.; Xiao, M. Enhanced TEA sensing properties of nest-like ZnO by decoration with Au. Mater. Res. Exp. 2019, 6, 105910. [CrossRef] 
343. Zhang, Y.-H.; Wang, C.-N.; Gong, F.-L.; Wang, P.; Guharoy, U.; Yang, C.; Zhang, H.-L.; Fang, S.-M.; Liu, J. Ultrathin agaric-like $\mathrm{ZnO}$ with Pd dopant for aniline sensor and DFT investigation. J. Hazard. Mater. 2020, 388, 122069. [CrossRef] [PubMed]

344. Liang, Y.; Yang, Y.; Xu, K.; Yu, T.; Peng, Q.; Yao, S.; Yuan, C. Controllable preparation of faceted $\mathrm{Co}_{3} \mathrm{O}_{4}$ nanocrystals@MnO $_{2}$ nanowires shish-kebab structures with enhanced triethylamine sensing performance. Sens. Actuators B 2020, $304,127358$. [CrossRef]

345. Peng, R.; Li, Y.; Chen, J.; Si, P.; Feng, J.; Zhang, L.; Ci, L. Reduced graphene oxide wrapped Au@ZnO core-shell structure for highly selective triethylamine gas sensing application at a low temperature. Sens. Actuators A 2018, 283, 128-133. [CrossRef]

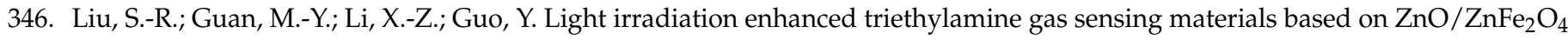
composites. Sens. Actuators B 2016, 236, 350-357. [CrossRef]

347. Xu, Y.; Ma, T.; Zheng, L.; Sun, L.; Liu, X.; Zhao, Y.; Zhang, J. Rational design of $\mathrm{Au} / \mathrm{Co}_{3} \mathrm{O}_{4}$-functionalized $\mathrm{W}_{18} \mathrm{O}_{49}$ hollow heterostructures with high sensitivity and ultralow limit for triethylamine detection. Sens. Actuators B 2019, 284, $202-212$. [CrossRef]

348. Liu, C.; Xu, H.; Chen, Z.; Ye, Q.; Wu, X.; Wang, J.; Cao, B. Enhanced Triethylamine Sensing Properties by Designing an $\alpha-\mathrm{Fe}_{2} \mathrm{O}_{3} / \alpha-\mathrm{MoO}_{3}$ Nanostructure Directly Grown on Ceramic Tubes. ACS Appl. Nano Mater. 2019, 2, 6715-6725. [CrossRef]

349. Li, H.; Zhang, N.; Zhao, X.; Xu, Z.; Zhang, Z.; Wang, Y. Modulation of TEA and methanol gas sensing by ion-exchange based on a sacrificial template 3D diamond-shaped MOF. Sens. Actuators B 2020, 315, 128136. [CrossRef]

350. Sun, L.; Sun, J.; Han, N.; Liao, D.; Bai, S.; Yang, X.; Luo, R.; Li, D.; Chen, A. rGO decorated W doped BiVO 4 novel material for sensing detection of trimethylamine. Sens. Actuators B 2019, 298, 126749. [CrossRef]

351. Chen, Z.; Xu, H.; Liu, C.; Cao, D.; Ye, Q.; Wu, X.; Wang, J.; Cao, B. Good triethylamine sensing properties of Au@MoS 2 nanostructures directly grown on ceramic tubes. Mater. Chem. Phys. 2020, 245, 122683. [CrossRef]

352. Chen, M.; Zhang, Y.; Lv, T.; Li, K.; Zhu, Z.; Zhang, J.; Zhang, R.; Liu, Q. Ag-LaFeO 3 nanoparticles using molecular imprinting technique for selective detection of xylene. Mater. Res. Bull. 2018, 107, 271-279. [CrossRef]

353. Chen, M.; Zhang, Y.; Zhang, J.; Li, K.; Lv, T.; Shen, K.; Zhu, Z.; Liu, Q. Facile lotus-leaf-templated synthesis and enhanced xylene gas sensing properties of $\mathrm{Ag}-\mathrm{LaFeO}_{3}$ nanoparticles. J. Mater. Chem. C 2018, 6, 6138-6145. [CrossRef]

354. Suematsu, K.; Watanabe, K.; Tou, A.; Sun, Y.; Shimanoe, K. Ultraselective Toluene-Gas Sensor: Nanosized Gold Loaded on Zinc Oxide Nanoparticles. Anal. Chem. 2018, 90, 1959-1966. [CrossRef]

355. Kang, Y.; Kim, K.; Cho, B.; Kwak, Y.; Kim, J. Highly Sensitive Detection of Benzene, Toluene, and Xylene Based on CoPPFunctionalized $\mathrm{TiO}_{2}$ Nanoparticles with Low Power Consumption. ACS Sens. 2020, 5, 754-763. [CrossRef] [PubMed]

356. Park, M.S.; Lee, J.H.; Park, Y.; Yoo, R.; Park, S.; Jung, H.; Kim, W.; Lee, H.-S.; Lee, W. Doping effects of ZnO quantum dots on the sensitive and selective detection of acetylene for dissolved-gas analysis applications of transformer oil. Sens. Actuators B 2019, 299, 126992. [CrossRef]

357. Xu, T.; Xu, P.; Zheng, D.; Yu, H.; Li, X. Metal-Organic Frameworks for Resonant-Gravimetric Detection of Trace-Level Xylene Molecules. Anal. Chem. 2016, 88, 12234-12240. [CrossRef] [PubMed]

358. Wang, T.; Huang, Z.; Yu, Z.; Wang, B.; Wang, H.; Sun, P.; Suo, H.; Gao, Y.; Sun, Y.; Li, T.; et al. Low operating temperature toluene sensor based on novel $\alpha-\mathrm{Fe}_{2} \mathrm{O}_{3} / \mathrm{SnO}_{2}$ heterostructure nanowire arrays. RSC Adv. 2016, 6, 52604-52610. [CrossRef]

359. Bang, J.H.; Choi, M.S.; Mirzaei, A.; Han, S.; Lee, H.Y.; Choi, S.-W.; Kim, S.S.; Kim, H.W. Hybridization of silicon nanowires with $\mathrm{TeO}_{2}$ branch structures and Pt nanoparticles for highly sensitive and selective toluene sensing. Appl. Surf. Sci. 2020, 525, 146620. [CrossRef]

360. Dhawale, D.S.; Gujar, T.P.; Lokhande, C.D. $\mathrm{TiO}_{2}$ Nanorods Decorated with Pd Nanoparticles for Enhanced Liquefied Petroleum Gas Sensing Performance. Anal. Chem. 2017, 89, 8531-8537. [CrossRef]

361. Lee, K.; Baek, D.-H.; Choi, J.; Kim, J. Suspended CoPP-ZnO nanorods integrated with micro-heaters for highly sensitive VOC detection. Sens. Actuators B 2018, 264, 249-254. [CrossRef]

362. Qin, H.; Cao, Y.; Xie, J.; Xu, H.; Jia, D. Solid-state chemical synthesis and xylene-sensing properties of $\alpha-\mathrm{MoO}_{3}$ arrays assembled by nanoplates. Sens. Actuators B 2017, 242, 769-776. [CrossRef]

363. Qin, H.; Xie, J.; Xu, H.; Li, Y.; Cao, Y. Green solid-state chemical synthesis and excellent xylene-detecting behaviors of Y-doped $\alpha-\mathrm{MoO}_{3}$ nanoarrays. Mater. Res. Bull. 2017, 93, 256-263. [CrossRef]

364. Koo, W.-T.; Choi, S.-J.; Kim, S.-J.; Jang, J.-S.; Tuller, H.L.; Kim, I.-D. Heterogeneous Sensitization of Metal-Organic Framework Driven Metal@Metal Oxide Complex Catalysts on an Oxide Nanofiber Scaffold Toward Superior Gas Sensors. J. Am. Chem. Soc. 2016, 138, 13431-13437. [CrossRef] [PubMed]

365. Vijayakumar, Y.; Mani, G.K.; Ponnusamy, D.; Shankar, P.; Kulandaisamy, A.J.; Tsuchiya, K.; Rayappan, J.B.B.; Ramana Reddy, M.V. $\mathrm{V}_{2} \mathrm{O}_{5}$ nanofibers: Potential contestant for high performance xylene sensor. J. Alloys Compd. 2018, 731, 805-812. [CrossRef]

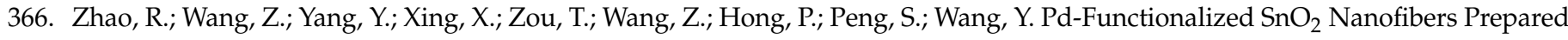
by Shaddock Peels as Bio-Templates for High Gas Sensing Performance toward Butane. Nanomaterials 2019, 9, 13. [CrossRef] [PubMed]

367. Kwon, Y.J.; Na, H.G.; Kang, S.Y.; Choi, S.-W.; Kim, S.S.; Kim, H.W. Selective detection of low concentration toluene gas using Pt-decorated carbon nanotubes sensors. Sens. Actuators B 2016, 227, 157-168. [CrossRef]

368. Seekaew, Y.; Wisitsoraat, A.; Phokharatkul, D.; Wongchoosuk, C. Room temperature toluene gas sensor based on TiO 2 nanoparticles decorated 3D graphene-carbon nanotube nanostructures. Sens. Actuators B 2019, 279, 69-78. [CrossRef] 
369. Du, L.; Song, X.; Liang, X.; Liu, Y.; Zhang, M. Formation of $\mathrm{NiCo}_{2} \mathrm{O}_{4}$ hierarchical tubular nanostructures for enhanced xylene sensing properties. Appl. Surf. Sci. 2020, 526, 146706. [CrossRef]

370. Xu, R.; Zhang, N.; Sun, L.; Chen, C.; Chen, Y.; Li, C.; Ruan, S. One-step synthesis and the enhanced xylene-sensing properties of Fe-doped $\mathrm{MoO}_{3}$ nanobelts. RSC Adv. 2016, 6, 106364-106369. [CrossRef]

371. Wang, B.; Jin, H.T.; Zheng, Z.Q.; Zhou, Y.H.; Gao, C. Low-temperature and highly sensitive $\mathrm{C}_{2} \mathrm{H}_{2}$ sensor based on Au decorated $\mathrm{ZnO} / \mathrm{In}_{2} \mathrm{O}_{3}$ belt-tooth shape nano-heterostructures. Sens. Actuators B 2017, 244, 344-356. [CrossRef]

372. Qu, F.; Jiang, H.; Yang, M. Designed formation through a metal organic framework route of $\mathrm{ZnO} / \mathrm{ZnCo}_{2} \mathrm{O}_{4}$ hollow core-shell nanocages with enhanced gas sensing properties. Nanoscale 2016, 8, 16349-16356. [CrossRef]

373. Li, F.; Li, C.; Zhu, L.; Guo, W.; Shen, L.; Wen, S.; Ruan, S. Enhanced toluene sensing performance of gold-functionalized $\mathrm{WO}_{3} \cdot \mathrm{H}_{2} \mathrm{O}$ nanosheets. Sens. Actuators B 2016, 223, 761-767. [CrossRef]

374. Gautam, C.; Tiwary, C.S.; Machado, L.D.; Jose, S.; Ozden, S.; Biradar, S.; Galvao, D.S.; Sonker, R.K.; Yadav, B.C.; Vajtai, R.; et al. Synthesis and porous h-BN 3D architectures for effective humidity and gas sensors. RSC Adv. 2016, 6, 87888-87896. [CrossRef]

375. Qiu, L.; Zhang, S.; Huang, J.; Wang, C.; Zhao, R.; Qu, F.; Wang, P.; Yang, M. Highly selective and sensitive xylene sensors based on $\mathrm{Nb}$-doped NiO nanosheets. Sens. Actuators B 2020, 308, 127520. [CrossRef]

376. Xia, W.; Liu, Y.; Li, J.; Chen, C. Investigation of CdO hexagonal nanoflakes synthesized by a hydrothermal method for liquefied petroleum gas detection. Anal. Methods 2016, 8, 6265-6269. [CrossRef]

377. Wang, D.; Yin, Y.; Xu, P.; Wang, F.; Wang, P.; Xu, J.; Wang, X.; Li, X. The catalytic-induced sensing effect of triangular CeO 2 nanoflakes for enhanced BTEX vapor detection with conventional ZnO gas sensors. J. Mater. Chem. A 2020, 8, 11188-11194. [CrossRef]

378. Dong, C.; Liu, X.; Xiao, X.; Du, S.; Wang, Y. Monodisperse $\mathrm{ZnFe}_{2} \mathrm{O}_{4}$ nanospheres synthesized by a nonaqueous route for a highly slective low-ppm-level toluene gas sensor. Sens. Actuators B 2017, 239, 1231-1236. [CrossRef]

379. Lee, K.H.; Kim, B.-Y.; Yoon, J.-W.; Lee, J.-H. Extremely selective detection of ppb levels of indoor xylene using $\mathrm{CoCr}_{2} \mathrm{O}_{4} \mathrm{hollow}$ spheres activated by Pt doping. Chem. Commun. 2019, 55, 751-754. [CrossRef]

380. Yao, L.; Li, Y.; Ran, Y.; Yang, Y.; Zhao, R.; Su, L.; Kong, Y.; Ma, D.; Chen, Y.; Wang, Y. Construction of novel Pd-SnO 2 composite nanoporous structure as a high-response sensor for methane gas. J. Alloys Compd. 2020, 826, 154063. [CrossRef]

381. Zhang, J.; Tang, P.; Liu, T.; Feng, Y.; Blackman, C.; Li, D. Facile synthesis of mesoporous hierarchical $\mathrm{Co}_{3} \mathrm{O}_{4}-\mathrm{TiO}_{2} \mathrm{p}-\mathrm{n} \mathrm{heterojunc-}$ tions with greatly enhanced gas sensing performance. J. Mater. Chem. A 2017, 5, 10387-10397. [CrossRef]

382. Sui, L.; Zhang, X.; Cheng, X.; Wang, P.; Xu, Y.; Gao, S.; Zhao, H.; Huo, L. Au-Loaded Hierarchical MoO 3 Hollow Spheres with Enhanced Gas-Sensing Performance for the Detection of BTX (Benzene, Toluene, And Xylene) And the Sensing Mechanism. ACS Appl. Mater. Interfaces 2017, 9, 1661-1670. [CrossRef]

383. Yang, L.; Zhou, X.; Song, L.; Wang, Y.; Wu, X.; Han, N.; Chen, Y. Noble Metal/Tin Dioxide Hierarchical Hollow Spheres for Low-Concentration Breath Methane Sensing. ACS Appl. Nano Mater. 2018, 1, 6327-6336. [CrossRef]

384. Kim, B.-Y.; Ahn, J.H.; Yoon, J.-W.; Lee, C.-S.; Kang, Y.C.; Abdel-Hady, F.; Wazzan, A.A.; Lee, J.-H. Highly Selective Xylene Sensor Based on $\mathrm{NiO} / \mathrm{NiMoO}_{4}$ Nanocomposite Hierarchical Spheres for Indoor Air Monitoring. ACS Appl. Mater. Interfaces 2016, 8 , 34603-34611. [CrossRef] [PubMed]

385. Zhang, R.; Gao, S.; Zhou, T.; Tu, J.; Zhang, T. Facile preparation of hierarchical structure based on $\mathrm{p}$-type $\mathrm{Co}_{3} \mathrm{O}_{4}$ as toluene detecting sensor. Appl. Surf. Sci. 2020, 503, 144167. [CrossRef]

386. Wei, Z.; Zhou, Q.; Lu, Z.; Xu, L.; Gui, Y.; Tang, C. Morphology controllable synthesis of hierarchical $\mathrm{WO}_{3}$ nanostructures and $\mathrm{C}_{2} \mathrm{H}_{2}$ sensing properties. Phys. E Low-Dimens. Syst. Nanostruct. 2019, 109, 253-260. [CrossRef]

387. Sonawane, N.B.; Baviskar, P.K.; Ahire, R.R.; Sankapal, B.R. CdO necklace like nanobeads decorated with PbS nanoparticles: Room temperature LPG sensor. Mater. Chem. Phys. 2017, 191, 168-172. [CrossRef]

388. Zhang, Y.; Bai, J.; Zhou, L.; Liu, D.; Liu, F.; Liang, X.; Gao, Y.; Liu, F.; Yan, X.; Lu, G. Preparation of silver-loaded titanium dioxide hedgehog-like architecture composed of hundreds of nanorods and its fast response to xylene. J. Colloid Interface Sci. 2019, 536, 215-223. [CrossRef]

389. Lu, S.; Hu, X.; Zheng, H.; Qiu, J.; Tian, R.; Quan, W.; Min, X.; Ji, P.; Hu, Y.; Cheng, S.; et al. Highly Selective, ppb-Level Xylene Gas Detection by $\mathrm{Sn}^{2+}$-Doped $\mathrm{NiO}$ Flower-Like Microspheres Prepared by a One-Step Hydrothermal Method. Sensors 2019, 19, 2958. [CrossRef]

390. Xu, H.; Li, J.; Fu, Y.; Tian, Y.; Yang, Z. Sensitized mechanism of recovered $\mathrm{S}-\mathrm{SnO}_{2}$ from tin sludge for $\mathrm{CH}_{4}$ detection by increasing oxygen vacancy density as an efficient strategy. Sens. Actuators B 2019, 298, 126838. [CrossRef]

391. Bai, S.; Du, L.; Sun, J.; Luo, R.; Li, D.; Chen, A.; Liu, C.-C. Preparation of reduced graphene oxide $/ \mathrm{Co}_{3} \mathrm{O}_{4}$ composites and sensing performance to toluene at low temperature. RSC Adv. 2016, 6, 60109-60116. [CrossRef]

392. Chen, N.; Deng, D.; Li, Y.; Xing, X.; Liu, X.; Xiao, X.; Wang, Y. The xylene sensing performance of $\mathrm{WO}_{3}$ decorated anatase TiO 2 nanoparticles as a sensing material for a gas sensor at a low operating temperature. RSC Adv. 2016, 6, 49692-49701. [CrossRef]

393. Zhang, Y.; Rong, Q.; Zhao, J.; Zhang, J.; Zhu, Z.; Liu, Q. Boron-doped graphene quantum dot/Ag-LaFeO 3 p-p heterojunctions for sensitive and selective benzene detection. J. Mater. Chem. A 2018, 6, 12647-12653. [CrossRef]

394. Subin David, S.P.; Veeralakshmi, S.; Sandhya, J.; Nehru, S.; Kalaiselvam, S. Room temperature operatable high sensitive toluene gas sensor using chemiresistive $\mathrm{Ag} / \mathrm{Bi}_{2} \mathrm{O}_{3}$ nanocomposite. Sens. Actuators B 2020, 320, 128410. [CrossRef]

395. Sukee, A.; Alharbi, A.A.; Staerz, A.; Wisitsoraat, A.; Liewhiran, C.; Weimar, U.; Barsan, N. Effect of AgO loading on flame-made $\mathrm{LaFeO}_{3}$ p-type semiconductor nanoparticles to acetylene sensing. Sens. Actuators B 2020, 312, 127990. [CrossRef] 
396. Hermawan, A.; Zhang, B.; Taufik, A.; Asakura, Y.; Hasegawa, T.; Zhu, J.; Shi, P.; Yin, S. CuO Nanoparticles/Ti ${ }_{3} \mathrm{C}_{2}$ Tx MXene Hybrid Nanocomposites for Detection of Toluene Gas. ACS Appl. Nano Mater. 2020, 3, 4755-4766. [CrossRef]

397. Behi, S.; Bohli, N.; Casanova-Cháfer, J.; Llobet, E.; Abdelghani, A. Metal Oxide Nanoparticle-Decorated Few Layer Graphene Nanoflake Chemoresistors for the Detection of Aromatic Volatile Organic Compounds. Sensors 2020, 20, 3413. [CrossRef]

398. Luo, S.-X.L.; Lin, C.-J.; Ku, K.H.; Yoshinaga, K.; Swager, T.M. Pentiptycene Polymer/Single-Walled Carbon Nanotube Complexes: Applications in Benzene, Toluene, and o-Xylene Detection. ACS Nano 2020, 14, 7297-7307. [CrossRef]

399. Ma, L.; Ma, S.Y.; Qiang, Z.; Xu, X.L.; Chen, Q.; Yang, H.M.; Chen, H.; Ge, Q.; Zeng, Q.Z.; Wang, B.Q. Preparation of Co-doped $\mathrm{LaFeO}_{3}$ nanofibers with enhanced acetic acid sensing properties. Mater. Lett. 2017, 200, 47-50. [CrossRef]

400. Zhu, Y.; Zhao, Y.; Ma, J.; Cheng, X.; Xie, J.; Xu, P.; Liu, H.; Liu, H.; Zhang, H.; Wu, M.; et al. Mesoporous Tungsten Oxides with Crystalline Framework for Highly Sensitive and Selective Detection of Foodborne Pathogens. J. Am. Chem. Soc. 2017, 139, 10365-10373. [CrossRef]

401. Abdul Hamid, H.; Lockman, Z.; Hattori, T.; Abdul Razak, K. Sensitive and selective chloroform sensor using $\mathrm{Fe}_{2} \mathrm{O}_{3}$ nanoparticledecorated ZnO nanorods in an aqueous solution. J. Mater. Sci. Mater. Electron. 2019, 30, 18990-19000. [CrossRef]

402. Yin, Y.; Zhang, N.; Han, J.; Liu, C.; Adimi, S.; Wen, S.; Li, X.; Ruan, S. Metal-organic framework derived core-shell PrFeO3functionalized $\alpha-\mathrm{Fe}_{2} \mathrm{O}_{3}$ nano-octahedrons as high performance ethyl acetate sensors. Sens. Actuators B 2019, $297,126738$. [CrossRef]

403. Gao, R.; Cheng, X.; Gao, S.; Zhang, X.; Xu, Y.; Zhao, H.; Huo, L. Highly selective detection of saturated vapors of abused drugs by ZnO nanorod bundles gas sensor. Appl. Surf. Sci. 2019, 485, 266-273. [CrossRef]

404. Kumar, T.H.V.; Raman Pillai, S.K.; Chan-Park, M.B.; Sundramoorthy, A.K. Highly selective detection of an organophosphorus pesticide, methyl parathion, using Ag-ZnO-SWCNT based field-effect transistors. J. Mater. Chem. C 2020, 8, 8864-8875. [CrossRef]

405. Chen, Q.; Li, J.; Fu, W.; Yang, Y.; Zhu, W.; Zhang, J. Detection of N,N-dimethylformamide vapor down to ppb level using electrospun InYbO nanofibers field-effect transistor. Sens. Actuators B 2020, 323, 128676. [CrossRef]

406. He, L.; Gao, C.; Yang, L.; Zhang, K.; Chu, X.; Liang, S.; Zeng, D. Facile synthesis of $\mathrm{MgGa}_{2} \mathrm{O}_{4}$ /graphene composites for room temperature acetic acid gas sensing. Sens. Actuators B 2020, 306, 127453. [CrossRef] 